\title{
ESTUDOS TAXONÔMICOS DE TRIPES (THYSANOPTERA) CONSTATADOS NO BRASIL, COM ÊNFASE NO GÊNERO Frankliniella
}

\author{
Renata ChIARIN MonteIRo \\ Engenheira Agrônoma
}

Orientadores: Prof. Dr. ROBERTO A. ZUCCHI Co-orientador: Prof. Dr. LAURENCE A. MOUND

\footnotetext{
Tese apresentada à Escola Superior de Agricultura

"Luiz de Queiroz", Universidade de São Paulo, para obtenção do título de Doutor em Ciências, Área de Concentração: Entomologia.
}

\section{P I R A C I C A B A}

Estado de São Paulo - Brasil

Novembro - 1999 
Dados Internacionais de Catalogação na Publicação (CIP) DIVISÃo DE BIBLIOTECA E DOCUMENTAÇÃo - Campus "Luiz de Queiroz"/USP

\author{
Monteiro, Renata Chiarini \\ Estudos taxonômicos de tripes (Thysanoptera) constatados no Brasil, com ênfase no \\ gênero Frankliniella / Renata Chiarini Monteiro. - - Piracicaba, 1999.
}

p.

Tese (doutorado) - - Escola Superior de Agricultura Luiz de Queiroz, 1999.

Bibliografia

1. Classificaçāo (biologia) 2. inseto-nocivo 3. Praga agricola 4. Tripes I. Título

CDD 632.731

"Permitida a cópia total ou parcial deste documento, desde que citada a fonte - O Autor: 
A minha família,

pelas oportunidades, apoio,

incentivo e carinho,

OFEREÇO.

Ao meu marido e filho,

Fernando e Pedro,

pelos sentimentos mais lindos, com carinho, 


\section{AGRADECIMENTOS}

Ao prof. Dr. Roberto A. Zucchi e ao Dr. Laurence A. Mound, pelos ensinamentos, orientação e incentivo.

Ao Dr. Sueo Nakahara, pelos ensinamentos, constante participação e acompanhamento.

Ao Dr. Charles L. Cole, pela disposição constante em ajudar, uso de laboratório e equipamentos e amizade.

Aos Drs. Robert $A$. Wharton e S. Bradley Vinson, pela uso de laboratório e equipamentos.

Aos Drs. Richard zur Strassen, Tamotsu Murai e Akira Kawai, pelas informações e trabalhos.

A todos os professores do Departamento de Entomologia, pelos ensinamentos e convivência.

Aos colegas e funcionários do Departamento de Entomologia, pela convivência e amizade.

As minhas amigas, em especial à Ardabil, Cláudia e Patrícia, pelo incentivo e carinho. Ao CNPq, pela bolsa de estudos concedida. 


\section{SUMÁRIO}

Página

LISTA DE FIGURAS ......................................................................................... vi

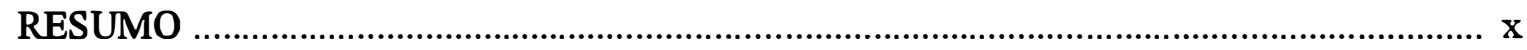

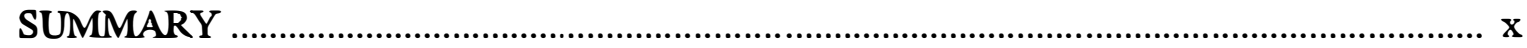

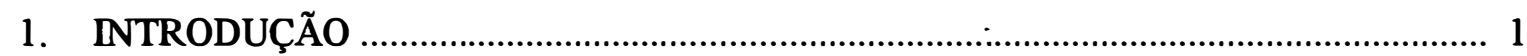

2. REVISÃO DE LITERATURA ….................................................................... 3

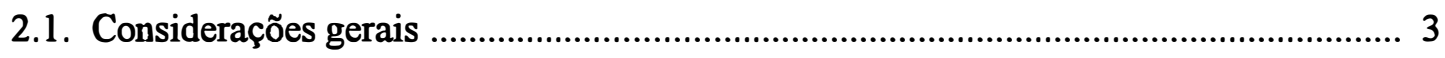

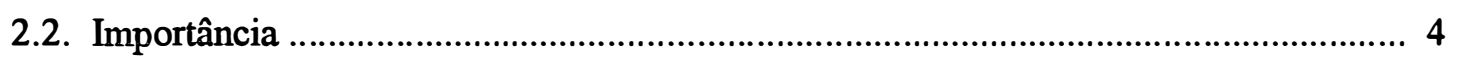

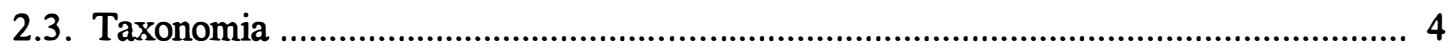

3. LISTA DAS ESPÉCIES DE THYSANOPTERA CONSTATADAS NO BRASIL ........... 6

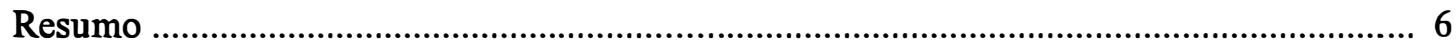

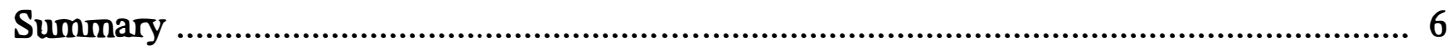

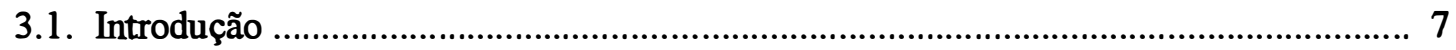

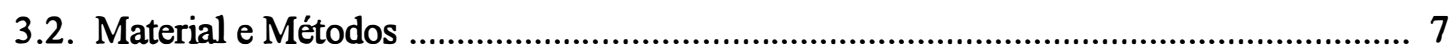

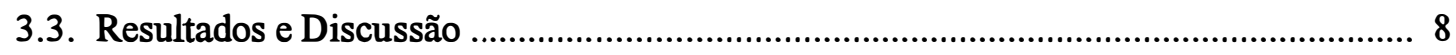

3.3.1. Lista de Terebrantia ............................................................................. 9

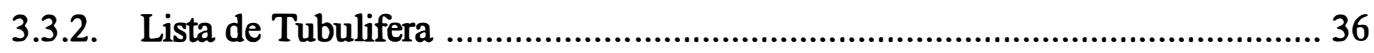

4. O GÊNERO Frankliniella NO BRASIL .................................................................. 78

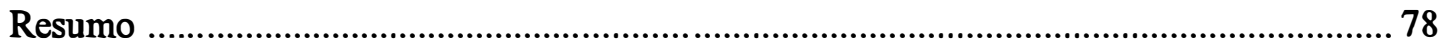

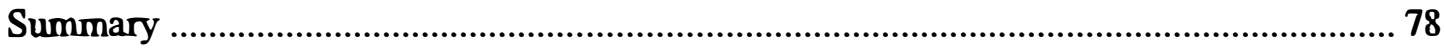

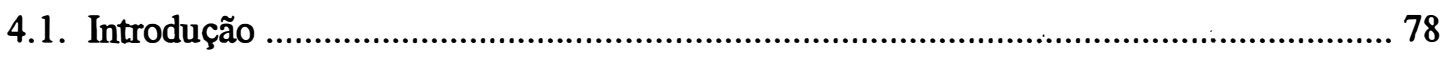

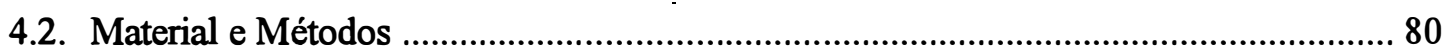

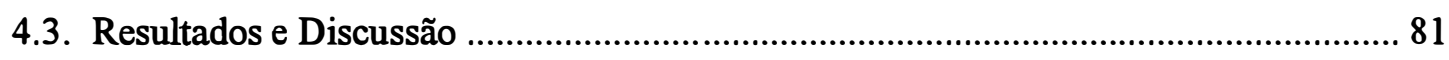

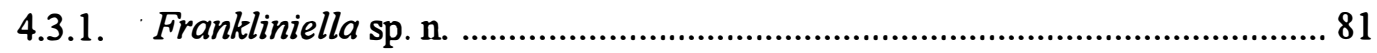

4.3.2. Chave de identificação das espécies de Frankliniella .................................... 84

4.3.3. Caracterizaçã morfológica das espécies de Frankliniella ............................. 90

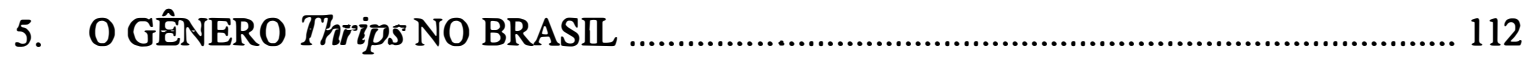

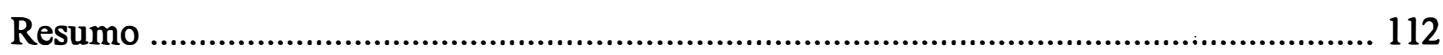

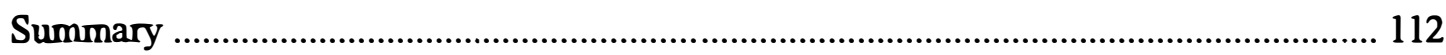

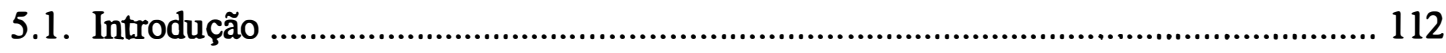




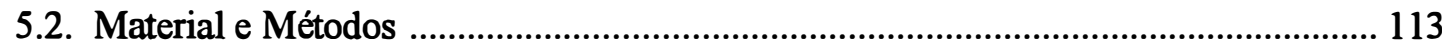

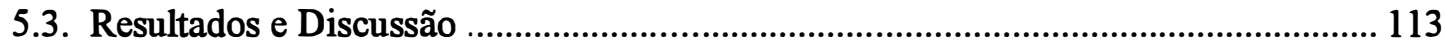

5.3.1. Chave de identificação das espécies de Thrips ........................................... 114

5.3.2. Caracterização morfológica das espécies de Thrips ................................... 115

6. GÊNEROS DE PANCHAETOTHRIPINAE NO BRASIL ............................................. 117

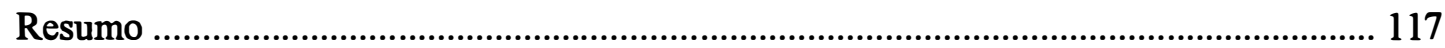

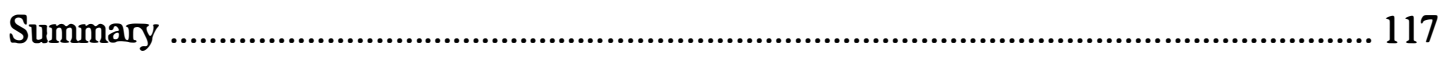

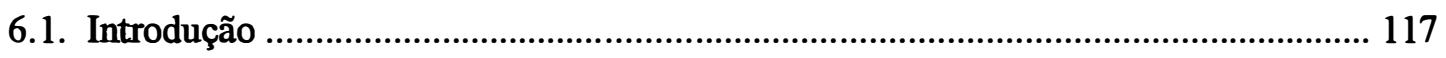

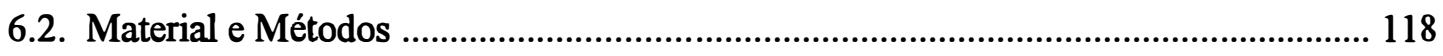

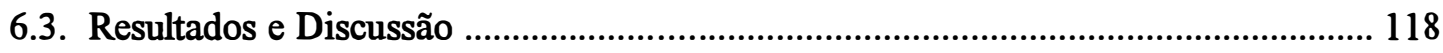

6.3.1. Chave de identificação dos gêneros de Panchaetothripinae ......................... 119

6.3.2. Caracterização morfológica dos gêneros de Panchaetothripinae .................. 120

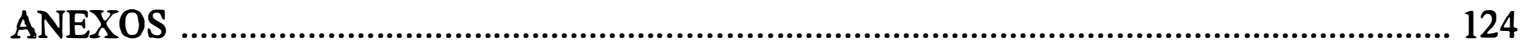

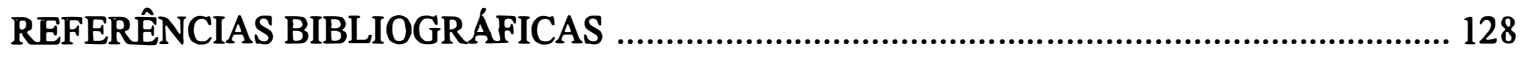

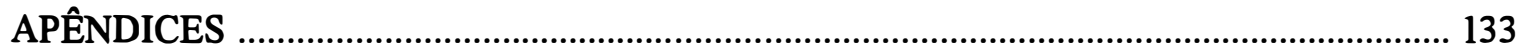




\section{LISTA DE FIGURAS E QUADRO}

I Figuras 1-14. Antena direita da + de Frankliniella spp. 1. F. annulipes; 2. F. bertelsi; 3. F. bondari; 4. F. brevicaulis; 5. F. brunnea; 6. F. condei; 7. F. desantisi; 8. F. distinguenda; 9. F. fulvipennis; 10. F. fulvipes; 11. F. gardenia; 12. F. gemina; 13. F. hemerocallis; 14. F. insularis.

II Figuras 15-27. Antena direita da $f$ de Frankliniella spp. 15. $F$. longipennis; 16. F. longispinosa; 17. F. occidentalis; 18. F. oxyura; 19. F. peruviana; 20. F. rodeos; 21. F. schultzei; 22. F. simplex; 23. F. trinidadensis; 24. F. varipes; 25. F. williamsi; 26. F. zucchini; 27. Frankliniella sp. $\mathbf{n}$.

III Figuras 15-27. Antena direita da $\&$ de Frankliniella spp. 15. $F$. longipennis; 16. F. longispinosa; 17. F. occidentalis; 18. F. oxyura; 19. F. peruviana; 20. F. rodeos; 21. F. schultzei; 22. F. simplex; 23. F. trinidadensis; 24. F. varipes; 25. F. williamsi; 26. F. zucchini; 27. Frankliniella sp. n.

IV Figuras 15-27. Cabeça (dorsal) da + de Frankliniella spp. 15. F. longipennis; 16. F. longispinosa; 17. F. occidentalis; 18. F. oxyura; 19. F. peruviana; 20. F. rodeos; 21. F. schultzei; 22. F. simplex; 23. F. trinidadensis; 24. F. varipes; 25. F. williamsi; 26. F. zucchini; 27. Frankliniella sp. n.

V Figuras 1-6. Pronoto da 9 de Frankliniella spp. 1. F. annulipes; 2. F. bertelsi; 3. F. bondari; 4. F. brevicaulis; 5. F. brunnea; 6. F. condei. ...

VI Figuras 7-14. Pronoto da 9 de Frankliniella spp. 7. F. desantisi; 8. F. distinguenda; 9. F. fulvipennis; 10. F. fulvipes; 11. F. gardenia; 12. F. gemina; 13. F. hemerocallis; 14. F. insularis. 
VII Figuras 15-22. Pronoto da 9 de Frankliniella spp. 15. F. longipennis; 16. F. longispinosa; 17. F. occidentalis; 18. $F$. oxyura; 19. $F$. peruviana; 20. F. rodeos; 21. F. schultzei; 22. F. simplex.

VIII Figuras 23-27. Pronoto da + de Frankliniella spp. 23. F. trinidadensis; 24. F. varipes; 25. F. williamsi; 26. F. zucchini; 27. Frankliniella sp. n.

IX Figuras 1-27. Metanoto da + de Frankliniella spp. 1. F. annulipes; 2. F. bertelsi; 3. F. bondari; 4. F. brevicaulis; 5. F. brunnea; 6. F. condei; 7. F. desantisi; 8. F. distinguenda; 9. F. fulvipennis; 10. F. fulvipes; 11. F. gardenia; 12. F. gemina; 13. $F$. hemerocallis; 14. F. insularis; 15. F. longipennis; 16. F. longispinosa; 17. F. occidentalis; 18. F. oxyura; 19. F. peruviana; 20. F. rodeos; 21. F. schultzei; 22. F. simplex; 23. F. trinidadensis; 24. F. varipes; 25. F. williamsi; 26. F. zucchini; 27. Frankliniella sp. n.

$\mathrm{X}$ Figuras 1-12. Tergito abdominal VIII da + de Frankliniella spp. 1. F. annulipes; 2. F. bertelsi; 3. F. bondari; 4. F. brevicaulis; 5. F. brunnea; 6. F. condei; 7. F. desantisi; 8. F. distinguenda; 9. F. fulvipennis; 10. F. fulvipes; 11. F. gardenia; 12. F. gemina.

XI Figuras 13-27. Tergito abdominal VIII da 9 de Frankliniella spp. 13. F. hemerocallis; 14. F. insularis; 15. F. longipennis; 16. F. longispinosa; 17. F. occidentalis; 18. F. oxyura; 19. F. peruviana; 20. F. rodeos; 21. F. schultzei; 22. F. simplex; 23. F. trinidadensis; 24. F. varipes; 25. F. williamsi; 26. F. zucchini; 27. Frankliniella sp. n.

XII Figuras 1-9. Tergito abdominal IX da $q$ de Frankliniella spp. 1. F. annulipes; 2. F. bertelsi; 3. F. bondari; 4. F. brevicaulis; 5. F. brunnea; 6. F. condei; 7. F. desantisi; 8. F. distinguenda; 9. F. fulvipennis.

XIII Figuras 10-17. Tergito abdominal IX da + de Frankliniella spp. 10. F. fulvipes; 11. F. gardenia; 12. F. gemina; 13. F. hemerocallis; 14. F. insularis; 15. F. longipennis; 16. F. longispinosa; 17. F. occidentalis. ...

XIV Figuras 18-27. Tergito abdominal IX da + de Frankliniella spp. 18. F. oxyura; 19. F. peruviana; 20. F. rodeos; 21. F. schultzei; 22. F. simplex; 23. F. trinidadensis; 24. F. varipes; 25. F. williamsi; 26. F. zucchini; 27. Frankliniella sp. n. 
1 Número de espécies e gêneros de Thysanoptera constatados no Brasil nas categorias Subordem, Família e Subfamília 


\title{
ESTUDOS TAXONÔMICOS DE TRIPES (THYSANOPTERA) CONSTATADOS NO BRASIL, COM ÊNFASE NO GÊNERO Frankliniella
}

\author{
Autor: RENATA CHIARINI MONTEIRO \\ Orientador: Prof. Dr. ROBERTO A. ZUCCHI \\ Co-Orientador: Dr. LAURENCE A. MOUND
}

\section{RESUMO}

Os estudos taxonômicos de Thysanoptera no Brasil foram desenvolvidos principalmente por pesquisadores norte-americanos e, conseqüentemente, suas coleções foram depositadas fora do País. Desse modo, não há uma coleção-referência formada, muito embora $70 \%$ das espécies constatadas no Brasil sejam descritas de espécimens nativos. Informações sobre a diversidade de espécies presentes no Brasil existem, porém muitas encontram-se esparsas em periódicos e coleções diversos ou englobam espécies além das registradas. Atualmente, 530 espécies de tripes foram constatadas no Brasil, 174 da Subordem Terebrantia e 356 de Tubulifera, o que representa cerca de $10 \%$ da diversidade conhecida. $O$ estudo apresenta uma lista das espécies de tripes válidas constatadas no Brasil, ordenadas nas categorias taxonômicas inclusivas (subordem, familia, subfamília e gênero) e chaves para a identificação de 27 espécies de Frankliniella, quatro espécies de Thrips e 10 gêneros de Panchaetothripinae, com ênfase à caracterização morfológica de Frankliniella spp., incluindo a descrição de uma nova espécie. 


\title{
TAXONOMIC STUDIES ON THRIPS (THYSANOPTERA) COLLECTED IN BRAZIL, WITH FOCUS ON THE GENUS Frankliniella
}

\author{
Author: RENATA CHIARINI MONTEIRO \\ Adviser: Prof. Dr. ROBERTO A. ZUCCHI \\ Co-Adviser: Dr. LAURENCE A. MOUND
}

\section{SUMMARY}

Taxonomic studies on Thysanoptera in Brazil were carried out mostly by North American researchers and, for this reason, the collections were not deposited here. There is no reference collection organized in Brazil, although $70 \%$ of the species collected here had been described from native specimens. There is information on species diversity that occurs in Brazil, however they are usually scaterred in many periodical publications and various collections or embrace more species than those recorded. Currently, from 530 thrips species collected in Brazil, 174 belong to Subordem Terebrantia and 356 to Tubulifera, adding up to $10 \%$ of total known diversity. This study presents a list of thrips valid species that were collected in Brazil, arranged in taxonomical categories (subordem, family, subfamily and genus) and keys to 27 Frankliella species, four Thrips species and 10 Panchaetothripinae genera, with focus on the morphological characterization of Frankliniella species. Also, a new species of Frankliniella is described. 


\section{INTRODUÇÃO}

No Brasil, há uma carência de estudos relacionados à identificação e biologia de insetos da Ordem Thysanoptera, conhecidos vulgarmente por tripes. Os estudos taxonômicos sobre tripes no Brasil foram desenvolvidos principalmente por J.D. Hood e D. Moulton na primeira metade deste século, com ênfase principalmente às espécies encontradas em serrapilheira de folhas. Hood descreveu espécies encontradas em todo continente americano, muitas delas do Brasil, mas não publicou chaves de identificacão, nem fez observações sobre a biologia (Hood, 1948; O'Neill, 1974). Em uma série de trabalhos entitulados "The Thysanoptera of South America" (Moulton, 1932, 1933a,b,c,d) e "Thysanoptera from Minas Geraes, Brazil" (Moulton, 1938, 1941), Moulton descreveu espécies, fez algumas observações biológicas e, embora tenha proposto chaves de identificação, utilizou um sistema de classificação não apropriado, baseado principalmente na coloração. $O$ material estudado por esses autores foi depositado em coleções nos EUA e, desse modo, não foi formada uma coleção de referência de Thysanoptera no Brasil. Mound \& Manullo (1996) listaram e elaboraram chaves para a identificação de espécies de tripes encontradas na Américas do Sul e, particularmente, Central, com informações sobre a biologia das mesmas. No entanto, as chaves e a listagem englobam um grande número de espécies, muitas não constatadas no Brasil, e abrangem principalmente a fauna da Costa Rica. Assim, no que se refere à fauna brasileira, não há uma lista de espécies e chaves específicas.

Dentre as espécies de tripes consideradas pragas, a maioria pertence à familia Thripidae, subfamílias Thripinae e Panchaetothripinae. Em Thripinae, dois gêneros, Thrips e Frankliniella, merecem destaque, por reunirem o maior número de espécies pragas, não apenas causadoras de danos diretos de alimentação, mas também transmissoras de fitopatógenos. Alguns Panchaeothripinae apresentam importância econômica, destacando-se, no Brasil, os gêneros Caliothrips, Heliothrips, Retithrips e Selenothrips. 
Os objetivos deste estudo foram listar as espécies de Thysanoptera, elaborar chaves de identificação para as espécies de Frankliniella e Thrips, da familia Thripinae, com ênfase no gênero Frankliniella, e dos gêneros de Panchaetothripinae constatados no Brasil e organizar uma coleção-referência da Ordem Thysanoptera no Brasil. 


\section{REVISĀO DE LITERATURA}

\subsection{Considerações gerais}

A principal característica morfológica dos membros da ordem Thysanoptera é a assimetria das partes bucais, cuja mandíbula direita é vestigial. As asas, quando presentes, apresentam uma franja marginal e as extremidades tarsais apresentam um órgão vesicular (arólio) eversível. Além dessas características, os tripes passam por uma metamorfose que engloba características da hemimetabolia e da holometabolia (Moritz, 1995), denominada remetabolia (Takahashi, 1921). A presença de franjas nas asas, embora seja o caráter morfológico que deu origem ao nome da Ordem, não é exclusivo dos tripes, uma vez que também é encontrada em representantes das Ordens Coleoptera (Ptilidae) e Hymenopera (Trichogrammatidae) (Lewis, 1973; Lewis, 1991).

Os adultos da Ordem Thysanoptera apresentam o corpo normalmente delgado e alongado, leve ou fortemente achatado dorso-ventralmente (Lewis, 1973) e com comprimento variável de 1 a 15 mm, embora a maioria das espécies raramente exceda 2mm (Heming, 1991).

Os tripes apresentam hábitos alimentares variáveis, podendo ser predadores, fitófagos ou fungívoros (esporos e hifas) (Lewis, 1973; Mound \& Teulon, 1995).

O ciclo de vida compreende as fases: ovo, dois estágios imaturos que se alimentam ativamente (ninfas I e II), dois (prepupa e pupa, em Terebrantia) ou três (prepupa, pupa I e pupa II, em Tubulifera) estágios quiescentes e adulto (Lewis, 1973, 1991; Heming, 1991). 


\subsection{Importância}

Os tripes podem ser importantes como agentes polinizadores (Kirk, 1988) e agentes de controle biológico, mas também podem ter "status" de praga, devido aos danos diretos causados pela alimentação (deformações nos tecidos, incluindo a formação de galhas, induzidas pelas toxinas da saliva), oviposição e deposição de fezes nos tecidos vegetais e/ou pelos danos indiretos (introdução de agentes fitopatogênicos, principalmente virus, ou abertura de uma "passagem", pela qual esses agentes possam penetrar nos tecidos vegetais) (Lewis, 1973; Mound, 1973). Frankliniella occidentalis, F. schultzei, F. zucchini, Thrips palmi e T. tabaci são vetoras de Tospovinus causadores de doenças em plantas.

\subsection{Taxonomia}

A Ordem Thysanoptera é dividida em duas subordens (Terebrantia e Tubulifera) e oito famílias, sete em Terebrantia (Aeolothripidae, Merothripidae, Heterothripidae, Thripidae, Uzelothripidae, Fauriellidae e Adiheterothripidae) e uma em Tubulifera (Phlaeothripidae) (Mound et al., 1980). Das oito famílias, apenas Fauriellidae e Adiheterothripidae não foram constatadas no Brasil.

As subordens são separadas rapidamente através da presença (Terebrantia) ou ausência (Tubulifera) de um ovipositor externo. Em Tubulifera, as asas anteriores, que se cruzam sobre o abdome quando em repouso, não apresentam nervuras e cerdas, exceto as da base, e suas superficies não têm microtríquias. Em Terebrantia, as asas anteriores, que se mantêm paralelas quando em repouso, apresentam nervuras com cerdas e suas superficies são cobertas com microtríquias (Mound \& Marullo, 1996).

As famílias mais comuns de Terebrantia (Aeolothripidae, Merothripidae, Heterothripidae, Thripidae) são diferenciadas através da curvatura do ovipositor em relação ao abdome, das asas anteriores (formato, largura, extremidade e venação) e do tipo de sensilo encontrado nos segmentos antenais III e IV. As fềmeas de Aeolothripidae possuem o ovipositor voltado em direção ao abdome e as asas anteriores largas e com o ápice arredondado. As outras três famílias diferem por apresentar o ovipositor voltado para baixo e as asas anteriores estreitas, com o ápice pontiagudo. $\mathrm{O}$ tipo de sensilo nos segmentos antenais III e IV é utilizado para separar 
essas três famílias. Em Merothripidae, além do ovipositor ser pouco desenvolvido, os sensilos são como tímpanos e circundam parcialmente a extremidade dos segmentos antenais III e IV. Heterothripidae e Thripidae apresentam o ovipositor bem desenvolvido. Em Heterothripidae, as áreas sensoriais são estreitas, compostas de muitos sensilos pequenos e arredondados, que circundam a extremidade dos segmentos antenais III e IV. Em Thripidae, os sensilos são estruturas simples ou furcadas, conhecidas como tricomas sensoriais (Palmer et al., 1898).

Na identificação de adultos de Thysanoptera são utilizados: coloração, medidas, forma, superficie e quetotaxia (cerdas e microtriquias) de diversas partes do corpo (Anexo 1). 


\section{LISTA DAS ESPÉCIES DE THYSANOPTERA CONSTATADAS NO BRASIL}

\section{RESUMO}

Há aproximadamente 5000 espécies de Thysanoptera, 530 constatadas no Brasil, pertencentes a seis famílias: Uzelothripidae, pela espécie única; Merothripidae, por nove espécies em dois gêneros; Aeolothripidae, por oito espécies em três gêneros; Heterothripidae, por 16 espécies em quatro gêneros; Thripidae-Panchaetothripinae, por 18 espécies em 10 gêneros; Thripidae-Thripinae, por 122 espécies em 37 gêneros; Phlaeothripidae-Idolothripinae, por 64 espécies em 17 gêneros e Phlaeothripidae-Phlaeothripinae, por 292 espécies em 66 gêneros.

\section{SUMMARY}

There are about 5000 species in Thysanoptera, from which 530 species are recorded in Brazil, belonging to six families: Uzelothripidae, the only species; Merothripidae, nine species in two genera; Aeolothripidae, eight species in three genera; Heterothripidae, 16 species in four genera; Thripidae-Panchaetothripinae, 18 species in 10 genera; Thripidae-Thripinae, 122 species in 37 genera; Phlaeothripidae-Idolothripinae, 64 species in 17genera and PhlaeothripidaePhlaeothripinae, 292 species in 66 genera. 


\subsection{Introdução}

A Ordem Thysanoptera apresenta cerca de 5000 espécies descritas, das quais mais de 100 foram relatadas como pragas de uma ou mais culturas (Mound, 1996). Estima-se a existência de outras 5000 espécies ainda não-descritas, principalmente nos trópicos (Lewis, 1973; Lewis, 1991). Gaston \& Mound (1993) relataram um total de 6479 nomes específicos em Thysanoptera, dos quais 5062 são aceitos e $22 \%$ são sinonímias.

Apesar de nos trópicos ocorrerem mais espécies de tripes que nas regiões temperadas, poucas são as informações publicadas sobre os Thysanoptera (Mound \& Marullo, 1996). No Brasil, mais de 500 espécies de tripes foram relatadas, o que representa cerca de $10 \%$ da fauna mundial conhecida (Mound \& Marullo, 1996).

Jacot-Guillarmod (1970-1979) e Jacot-Guillarmod \& Brothers (1986) catalogaram as espécies de tripes do mundo em uma obra extensa (7 volumes) e Silva et al. (1968) listaram 134 espécies de tripes associadas a plantas no Brasil. No entanto, parte dos registros encontrados em alguns trabalhos de Silva et al. (1968) não apresenta acompanhamento taxonômico ou coleçãoreferência (voucher specimens), tornando-os duvidosos. Mound \& Marullo (1996) realizaram um estudo mais direcionado às Américas Central e do Sul, reunindo informações biológicas e taxonômicas sobre os gêneros e espécies, atualizando e caracterizando os táxons. No entanto, não há uma listagem direcionada apenas ao Brasil, acessível e de uso prático.

\subsection{Material e Métodos}

A listagem das espécies de Thysanoptera constatadas no Brasil foi feita com base principalmente nas publicações de Hood (Hood, 1948; O'Neill, 1974), Moulton (1926, 1932, 1933a,b,c,d, 1935, 1938, 1941, 1948), Silva et al. (1968), Jacot-Guillarmod (1970-1979), JacotGuillarmod \& Brothers (1986), Mound \& Marullo (1996), informações pessoais do Dr. Mound, Dr. Nakahara, material depositado no "Seckenberg Museum, Frankfurt" e em dados do autor.

As espécies são listadas em ordem alfabética dentro das categorias taxonômicas: subordem, família, subfamilia (apenas em Thripidae) e gênero. 


\subsection{Resultados e Discussão}

Seis das oito famílias da Ordem Thysanoptera foram constatadas no Brasil, cinco da Subordem Terebrantia (Uzelothripidae, Merothripidae, Aelothripidae, Heterothripidae e Thripidae) e uma da Subordem Tubulifera (Phlaeothripidae). As famílias mais comuns sendo, Merothripidae, Heterothripidae, Thripidae e Phlaeothripidae.

Uzelothripidae está representada pela única espécie (Uzelothrips scabrosus); Merothripidae, por nove espécies em dois gêneros; Aeolothripidae, por oito espécies em três gêneros; Heterothripidae, por 16 espécies em 4 gêneros; Thripidae-Panchaetothripinae, por 18 espécies em 10 gêneros; Thripidae-Thripinae, por 122 espécies em 37 gêneros; PhaeothripidaeIdolothripinae, por 64 espécies em 17 gêneros; Phlaeothripidae-Phlaeothripinae, por 292 em 66 gêneros, totalizando 530 espécies, 374 descritas do Brasil (90 em Terebrantia, 52 em TubuliferaIdolothipinae e 232 em Tubulifera-Phlaeothripinae), o que representa aproximadamente $70 \%$ das espécies constatadas (Quadro 1).

\begin{tabular}{|c|c|c|c|c|c|}
\hline \multirow[t]{2}{*}{ SUBORDEM } & \multirow[t]{2}{*}{ FAMILIA } & \multirow[t]{2}{*}{ SUBFAMILIA } & \multirow[t]{2}{*}{ GÊNEROS } & \multicolumn{2}{|c|}{ ESPÉCIES } \\
\hline & & & & Total & $\begin{array}{c}\text { Descritas do } \\
\text { Brasil }\end{array}$ \\
\hline \multirow[t]{8}{*}{ TEREBRANTIA } & UZELOTHRIPIDAE & & 1 & 1 & 1 \\
\hline & MEROTHRIPIDAE & & 2 & 9 & 4 \\
\hline & AEOLOTHRIPIDAE & & 3 & 8 & 6 \\
\hline & HETEROTHRIPIDAE & & 4 & 16 & 16 \\
\hline & THRIPIDAE & Panchaeothripinae & 10 & 18 & 1 \\
\hline & & Thripinae & 37 & 122 & 62 \\
\hline & FAURIELIIDAE & & - & - & - \\
\hline & ADIHETEROTHRIPIDAE & & - & - & - \\
\hline \multirow[t]{2}{*}{ TUBULIFERA } & PHLAEOTHRIPIDAE & Idolothripinae & 17 & 64 & 52 \\
\hline & & Phlaeothripinae & 66 & 292 & 232 \\
\hline TOTAL & & & 140 & 530 & 374 \\
\hline
\end{tabular}

Quadro 1. Número de espécies e gêneros de Thysanoptera constatados no Brasil nas categonas Subordem, Familia e Subfamilia. 


\section{Lista dos Terebrantia (Thysanoptera) constatados no Brasil}

\section{UZELOTHRIPIDAE}

\section{Gênero Uzelothrips}

Uzelothrips scabrosus Hood, 1952: 143 (Brasil: Belém, PA) (ramos mortos de Hevea sp. e Bixa sp.)

Hood, J.D. 1952. Brasilian Thysanoptera III. Proceedings of the Biological Society of Washington, 65: 141-174.

Distribuicão no Brasil: PA (Belém) e sul Brasil

Hábitat: ramos mortos de Hevea sp. e Bixa sp. caidos no chão

\section{MEROTHRIPIDAE}

\section{Gênero Damerothrips}

Damerothrips gemmatus Hood, 1954: 19 (Brasil: Rondon, PR) (sem informação)

Hood, J.D. 1954. Brasilian Thysanoptera IV. Proceedings of the Biological Society of Washington, 67: 17-54.

Distribuição no Brasil: PR, MS

Hábitat: ramos mortos

\section{Gênero Merothrips}

Merothrips brevisetis Hood, 1954: 20 (Brasil: Belém, PA) (ramos mortos de Hevea sp. e Bixa sp.)

Hood, J.D. 1954. Brasilian Thysanoptera IV. Proceedings of the Biological Society of Washington, 67: 17-54.

Distribuicão no Brasil: PA (Belém)

Hábitat: ramos mortos de Hevea sp. e Bixa sp.

Merothrips brunneus Ward, 1969: 362 (Nova Zelândia)

Ward, A. 1969. Two new Merothrips (Thysanoptera - Merothripidae) from New Zealand. Records of the Canterbury Museum, 8: 359-366.

Distribuição no Brasil:

Hábitat:

Merothrips floridensis Watson, 1927: 60 (EUA: Earleton, Flórida) (musgo crescendo no tronco de uma árvore de carvalho viva)

Watson, J.R. 1927. New Thysanoptera from Florida. Florida Entomologist, 10(4): 60-62.

Distribuição no Brasil: SP

Hábitat:

Merothrips fusciceps Hood \& Williams, 1915: 123 (USA: New Orleans, Louisiana) (grupo de bambus omamentais)

Hood, J.D. \& C.B. Williams. 1915. New Thysanoptera from Florida and Louisiana. Journal of the New York Entomological Society, 23(2): 121-138 + plates 7-9.

Sinonimias: Merothrips cognatus Hood, 1925: 53 (Trinidade) (ramos mortos de Lagerstroemia infestado com bromélias)

Hood, J.D. 1925. New Neotropical Thysanoptera collected by C.B. Williams. Psyche, 32(1): 48-69.

Distribuição no Brasil: RJ (próximo ao Rio de Janeiro)

Hábitat: 
Merothrips mirus Crawford, 1942: 152 (Brasil: Nova Teutônia, SC) (ramos mortos)

Crawford, J.C. 1942. Two new South american species of Merothrips Hood (Thysanoptera, Merothripidae). Proceedings of the Entomological Society of Washington, 44: 150-154.

Distribuicão no Brasil: SC (Nova Teutônia)

Hábitat: ramos mortos

Merothrips morgami Hood, 1912: 132 (USA: Homer, Illinois) (sob a casca de árvore solta de nogueira)

Hood, J.D. 1912. Descriptions of new North American Thysanoptera. Proceedings of the Entomological Society of Washington, 14(3): 129-160 + 5 plates.

Sinônimo: Merothrips plaumanni Crawford, 1942: 150 (Brasil: Nova Teutônia, SC)

Crawford, J.F. 1942. Two new South American species of Merothrips Hood (Thysanoptera, Merothripidae). Proceedings of the Entomological Society of Washington, 44(7): 150-154.

Distribuicão no Brasil: SC (Nova Teutônia)

Hábitat:

Merothrips tympanis Hood, 1954: 20 (Brasil: Nova Teutônia, SC) (ramos mortos)

Hood, J.D. 1954. Brasilian Thysanoptera IV. Proceedings of the Biological Society of Washington, 67: 17-54.

Distribuicão no Brasil: SC (Nova Teutônia), PR (Rondon), MS

Hábitat: ramos mortos

Merothrips williamsi Priesner, 1921: 191 (Paraguai) (sob a casca das árvores)

Priesner, H. 1921. Neue und wenig bekannte Thysanopteren der neotropischen Fauna aus der Sammlung des Berliner Zoologischen Museums. Deutsche Entomologische Zeitung, 1921(3): 187-223.

Sinônimo: Merothrips genuinus Hood, 1938: 354 (EUA: Homestead, Flórida) (ramos mortos)

Hood, J.D. 1938. New Thysanoptera from Florida and North Carolina. Revista de Entomologia, 8(3-4): 348-420.

Distribuicão no Brasil: PA, PR, MS

Hábitat: sob a casca das árvores

\section{AEOLOTHRIPIDAE}

\section{Gênero Erythrothrips}

Erythrothrips brasiliensis Hood, 1952: 141 (Brasil, Nova Teutônia, SC) (Dalbergia sp.)

Hood, J.D. 1952. Brasilian Thysanoptera III. Proceedings of the Biological Society of Washington, 65: 141-174.

Distribuicão no Brasil: SC (Nova Teutônia)

Hábitat: Dalbergia sp.

Erythrothrips loripes Hood, 1957:129 (Brasil: Nova Teutônia, SC) (sem dado)

Hood, J.D. 1957. New Brazilian Thysanoptera. Proceedings of the Biological Society of Washington, 70: 129-180.

Distribuição no Brasil: SC (Nova Teutônia)

Hábitat: gramineas

\section{Gênero Franklinothrips}

Franklinothrips fulgidus Hood, 1949: 8 (Brasil: Petrópolis, RJ) (vegetação misturada)

Hood, J.D. 1949. Brasilian Thysanoptera I. Revista de Entomologia, 20(1-3): 3-88.

Distribuicão no Brasil: RJ (Petrópolis), SP (Fazenda Salto/São Carlos)

Hábitat: vegetação misturada; trepadeiras em arbustos 
Franklinothrips lineatus Hood, 1949: 4 (Brasil: Nova Teutônia, SC) (folhas de Vitex sp.)

Hood, J.D. 1949. Brasilian Thysanoptera I. Revista de Entomologia, 20(1-3): 3-88.

Distribuição no Brasil: SC (Nova Teutônia)

Hábitat: folhas de Vitex sp.

Franklinothrips tenuicornis Hood, 1915: 164 (Panamá: Baia do Panamá, Moro Island, próximo a Tobago) (coletando com rede)

Hood, J.D. 1915. On some American Aeolothripidae (Thysanoptera). Entomological News, 26(4): 162-166.

Distribuição no Brasil: MG, SP (Piracicaba)

Hábitat: hospedeiro desconhecido (coletando com rede); folhas de cafeeiro; predador de Selenothrips rubrocinctus; citros; planta daninha com flor lilás

Franklinothrips vespiformis (Crawford, 1909: 109) (Aeolothrips) (Nicarágua: Managua) (desconhecido) Crawford, J.C. 1909. Some Thysanoptera of Mexico and the south, I. Pomona College Journal of Entomology, 1(4): 109-119.

Distribuição no Brasil: BA, MG, SP (Piracicaba)

Hábitat: hospedeiro desconhecido (coletando com rede); folha de soja; folhas de mandioca; cacao

\section{Franklinothrips sp.}

Distribuicão no Brasil: SP (Piracicaba)

Hábitat: girassol

\section{Gênero Stomatothrips}

Stomatothrips angustipennis Hood, 1949: 12 (Brasil: Nova Teutônia, SC) (Panicum sp.; gramíneas e um ramo morto)

Hood, J.D. 1949. Brasilian Thysanoptera I. Revista de Entomologia 20(1-3): 3-88.

Distribuição no Brasil: RJ (Campo Grande, Jacarepaguá/Rio de Janeiro), SC (Nova Teutônia), SP (Piracicaba)

Hábitat: Panicum sp.; gramineas (folhas e base da toceira) e ramos mortos

Stomatothrips rotundus Hood, 1949: 10 (Brasil: Nova Teutônia, SC) (Panicum sp.)

Hood, J.D. 1949. Brasilian Thysanoptera I. Revista de Entomologia, 20(1-3): 3-88.

Distribuicão no Brasil: SC (Nova Teutônia), SP (Piracicaba)

Hábitat: Panicum sp.; milho e daninhas ao redor

\section{HETEROTHRIPIDAE}

\section{Gênero Aulacothrips}

Aulacothrips dictyotus Hood, 1952: 142 (Brasil: Nova Teutônia, SC) (Compositae e Solanum sp.)

Hood, J.D. 1952. Brasilian Thysanoptera III. Proceedings of the Biological Society of Washington, 65: 141-174.

Distribuicão no Brasil: SC (Nova Teutônia), SP

Hábitat: Compositae e Solanum sp.

\section{Gênero Heterothrips}

Heterothrips angusticeps Hood, 1954: 195 (Brasil: Nova Teutônia, SC) (desconhecido)

Hood, J.D. 1954. Brasilian Thysanoptera V. Proceedings of the Biological Society of Washington, 67: 195-214.

Distribuição no Brasil: SC (Nova Teutônia)

Hábitat: desconhecido 
Heterothrips bicolor Hood, 1954: 196 (Brasil: Nova Teutônia, SC) (desconhecido)

Hood, J.D. 1954. Brasilian Thysanoptera V. Proceedings of the Biological Society of Washington, 67: 195-214.

Distribuição no Brasil: SC (Nova Teutônia)

Hábitat: desconhecido

Heterothrips brasiliensis Moulton, 1932: 473 (Brasil: Santa Thereza, ES) (Beginia sp. e planta não identificada)

Moulton, D. 1932. The Thysanoptera of South America I. Revista de Entomologia, 2(4): 451-484.

Distribuição no Brasil: ES (Santa Thereza)

Hábitat: Beginia sp.; planta não identificada

Heterothrips condei Moulton, 1932: 472 (Brasil: Santa Thereza, ES) (desconhecido)

Moulton, D. 1932. The Thysanoptera of South America I. Revista de Entomologia, 2(4): 451-484.

Distribuicão no Brasil: ES (Santa Thereza)

Hábitat: desconhecido

Heterothrips decoratus Hood, 1954: 197 (Brasil: Nova Teutônia, SC) (Luehea sp.)

Hood, J.D. 1954. Brasilian Thysanoptera V. Proceedings of the Biological Society of Washington, 67: 195-214.

Distribuicãa no Brasil: SC (Nova Teutônia)

Hábitat: Luehea sp.

Heterothrips flavidus Hood, 1954: 198 (Brasil: Nova Teutônia, SC) (planta não identificada)

Hood, J.D. 1954. Brasilian Thysanoptera V. Proceedings of the Biological Society of Washington, 67: 195-214.

Distribuição no Brasil: SC (Nova Teutônia)

Hábitat: planta não identificada

Heterothrips flavitibia Moulton, 1932: 468 (Brasil: Santa Thereza, ES) (Serjania sp. ou Paullinia sp.)

Moulton, D. 1932. The Thysanoptera of South America I. Revista de Entomologia, 2(4): 451-484.

Distribuição no Brasil: ES (Santa Thereza), MG

Hábitat: Serjania sp. ou Paullinia sp.

Heterothrips marginatus Hood, 1954: 198 (Brasil: Belém, PA) (Andropogon sp. e ramos mortos)

Hood, J.D. 1954. Brasilian Thysanoptera V. Proceedings of the Biological Society of Washington, 67: 195-214.

Distribuicão no Brasil: PA (Belém), RJ (Campo Grande)

Hábitat: Andropogon sp. e ramos mortos

Heterothrips peixotoa Del-Claro, Marullo \& Mound, 1997: 1309 (Brasil: Uberlândia, MG) (flor de Peixotoa tomentosa)

Del-Claro, K; R. Marullo \& L.A. Mound. 1997. A new Brazilian species of Heterothrips (Insecta: Thysanoptera) co-exixting with ants in the flowers of Peixotoa tomentosa (Malpighiaceae). Joumal of Natural History, 31: 13071312.

Distribuicão no Brasil: MG (Uberlândia)

Hábitat: flores de Peixotoa tomentosa

Heterothrips semiflavus De Santis, 1972: 431 (Brasil: Barueri, SP) (ninhos de formiga Camponotus rufipis)

De Santis, L. 1972. Descripción preliminar de una nueva especie de Heterothrips del Brasil (Thysanoptera: Heterothripidae). Studia Entomológica, Revista International de Entomologia, 15(1-4): 431-432.

Distribuição no Brasil: SP (Barueri)

Hábitat: ninhos de formiga Camponotus rufipis

Heterothrips spinosus Moulton, 1932: 470 (Brasil: Colônia Rio Bonito, Santa Leopoldina, ES) (Solanum sp.) Moulton, D. 1932. The Thysanoptera of South America I. Revista de Entomologia, 2(4): 451-484.

Distribuição no Brasil: ES (Colônia Rio Bonito/Santa Leopoldina) 
Hábitat: Solanum sp.

Heterothrips striatus Moulton, 1932: 471 (Brasil: Santa Thereza, ES) (desconhecido)

Moulton, D. 1932. The Thysanoptera of South America I. Revista de Entomologia, 2(4): 451-484.

Distribuicão no Brasil: ES (Santa Thereza)

Hábitat: desconhecido

Heterothrips varitibia Moulton, 1932: 469 (Brasil: SP) (jurubeba)

Moulton, D. 1932. The Thysanoptera of South America I. Revista de Entomologia, 2(4): 451-484.

Distribuicão no Brasil: SP

Hábitat: jurubeba

\section{Gênero Lenkothrips}

Lenkothrips sensitivus (De Santis \& Sureda, 1970: 471) (Heterothrips) (Brasil: SP)

De Santis, L. \& A.E. Galego de Sureda. 1970. Un nuevo Heterothipido de Brasil (Thysanoptera). Studia Entomológica, Revista International de Entomologia, 13: 471-473.

Distribuição no Brasil: SP

Hábitat:

\section{Gênero Scutothrips}

Scutothrips nudus (Moulton, 1932: 474) (Heterothrips) (Brasil: Colônia Rio Bonito, Santa Leopoldina, ES) (Leguminasae)

Moulton, D. 1932. The Thysanoptera of South America I. Revista de Entomologia, 2(4): 451-484.

Sinônimos: Heterothrips moreirai Moulton, 1932: 467, 475 (Brasil: Santa Thereza, ES) (desconhecido)

Moulton, D. 1932. The Thysanoptera of South America I. Revista de Entomologia, 2(4): 451-484.

Distribuicão no Brasil: ES (Colônia Rio Bonito/Santa Leopoldina), RJ

Hábitat: Malpighiaceae; planta desconhecida

\section{THRIPIDAE - PANCHAETOTHRIPINAE}

\section{Gênero Arachisothrips}

Arachisothrips millsi Stannard, 1952: 330 (Jamaica: Hardwar Gap, Portland Parish) (serrapilheira e musgos no chão de um local semi-sombreado de uma floresta chuvosa, altitude 4000 pés)

Stannard, L. J. Jr. 1952. Peanut-winged thrips (Thysanoptera: Thripidae). Annals of the Entomological Society of America, 45(2): 327-330.

Distribuição no Brasil: SC (Nova Teutônia)

Hábitat: serrapilheira e musgos; serrapilheira composta principalmente por Selaginella sp.

\section{Gênero Brachyurothrips}

Brachyurothrips anomalus Bagnall, 1921: 265 (Thas Seychelles) (desconhecido)

Bagnall, R.S. 1921. On Thysanoptera from the Seychelles Islands and Rodrigues. Annals and Magazine of Natural History, 7(9): 257-293.

Sinônimos: Brachyurothrips walteri Costa Lima, 1957 ou 1956: 560 (Brasil: Salvador, BA) (pimenteira)

Costa Lima, A. M. da. 1957 ou 1956?. Sôbre um trips da pimenteira (Thysanoptera: Thripidae). Memórias do Instituto Oswaldo Cruz, 54(3): 559-563.

Distribuição no Brasil: BA (Salvador), SP

Hábitat: pimenteira 


\section{Gênero Caliothrips}

Caliothrips cinctipennis (Hood, 1912: 137) (Heliothrips) (USA: Makanda, Illinois) (coletando com rede em gramíneas, daninhas e outras plantas)

Hood, J.D. 1912. Descriptions of new North American Thysanoptera. Proceedings of the Entomological Society of Washington, 14(3): 129-160 + 5 plates

Distribuicão no Brasil: RJ

Hábitat: folhas de arroz, gramíneas e daninhas

Caliothrips fasciatus (Pergande, 1895: 391) (Heliothrips) (EUA: Matyville, Califomia) (laranja)

Pergande, T. 1895. Observations on certain Thiripidae. Insect Life. U.S. Department of Agriculture, Division of Entomology, 7: 390-395.

Distribuicão no Brasil: BA, CE, RJ, RS

Hábitat: um grande número de plantas

Caliothrips insularis (Hood, 1927: 234) (Hercothrips) (EUA: St. Croix) (graminea, coletando com rede)

Hood, J.D. 1927. New Thysanoptera collected by C.B. Williams. II. Psyche, 34(6): 230-246.

Sinônimo: Caliothrips bruneri Morgan, 1929: 8 (Heliothrips)

Morgan, A.C. 1929. A new genus and five new species of Thysanoptera foreign to the United States. Proceedings of the Entomological Society of Washington, 31(1): 1-9.

Distribuicão no Brasil: BA, SP

Hábitat:

Caliothrips phaseoli (Hood, 1912: 113) (Heliothrips) (EUA: Brownsville, Texas) (face inferior de folhas de feijão)

Hood, J.D. 1912. A new genus and three new species of North American Thysanoptera. Psyche, 19(4): 113-118.

Sinônimos:

Heliothrips braziliensis Morgan, 1929: 7 (Brasil: não mencionado) (não mencionado) (coletado por C. Moreira)

Morgan, A.C. 1929. A new genus and five new species of Thysanoptera foreign to the United States. Proceedings of the Entomological Society of Washington, 31(1): 1-9.

Heliothrips gossypii Moulton, 1927: 32 (EUA: Arizona) (algodão)

Moulton, D. 1927. Four new Californian Thysanoptera with notes on two other species. Pan-Pacific Entomologist,14(1): 30-35.

Hercothrips ipomoea Mouton, 1932: 481 (Brasil: Santa Thereza, ES) (Ageratum conizoides)

Moulton, D. 1932, The Thysanoptera of South America. I. Revista de Entomologia, 2(4): 451-484.

Caliothrips flavescentis De Santis, 1967: 3 (Brasil: Pelotas, RS) (Lycopersicum esculentum)

De Santis, L. 1967. Dos nuevas especies de Tisanopteros del Brasil. Comision de Investigacion Cientifica, La Plata, 4(6): 1-6.

Distribuicão no Brasil: BA, MG, PE (Santa Maria da Boa Vista), PR (Bandeirantes, Curitiba), RJ, RS (Santa Maria), SP (Araras, Ilha Solteira, Jaboticabal, Piracicaba, Campinas),

Hábitat: leguminosas; feijão (Phaseolus vulgaris); algodão; girassol; soja; batata (Ipomoeae batatas); tomate (Lycopersicum esculentum); folhas de pepino; cravo-de-defunto (Tagetes patula); Ageratum conizoides, alface; folha de cenoura

\section{Caliothrips sp.}

Distribuicão no Brasil: SP (Mata de Santa Genebra/Campinas, Piracicaba)

Hábitat: Ibantes sp. , base da touceira de gramíneas

\section{Gênero Dinurothrips}

Dinurothrips hookeri Hood, 1913: 151 (Porto Rico: Mayagüez) (Ipomoea sp.)

Hood, J.D. 1913. On a collection of Thysanoptera from Porto Rico. Insecutor Inscitiae Menstruus, 1(12): 149-154.

Distribuição no Brasil: BA, RJ, SP (Piracicaba), RCM 31, RCM 71 
Hábitat: folhas de Cestrum sp.; folhas de Erechites; Bidens sp.; folhas de maria-sem-vergonha (Impatiens balsamina); folhas de orquideas (Dendrobium sp. e Phalaenopsis sp.) em aclimatação em casa-de-vegetação; folhas de maria-pretinha (Solanum nigrum); folhas de batata doce; caruru (Amarranthus viridis); coerana preta; língua-devaca de flor amarela; Citrus sinensis

Dinurothrips vezenyii Bagnall, 1919: 256 (Argentina: Tucumán) (desconhecido)

Bagnall, R.S. 1919. Brief descriptions of new Thysanoptera. X. Annals and Magazine of Natural History, $9^{\text {th }}$ Series, 4(22): 253-277.

Sinônimos: Dinurothrips vanzenyii Bagnall, 1919: 256 (Argentina: Tucuman) (desconhecido) - escrito incorretamente por Hood, 1958 (Brasil: Nova Teutônia, SC)

Bagnall, R.S. 1919. Brief descriptions of new Thysanoptera. X. Annals and Magazine of Natural History, $9^{\text {th }}$ Series, 4(22): 253-277.

Hood, J.D. 1958. The rediscovery of Dinurothrips vanzenyii Bagnall (Thysanoptera: Thripidae). Florida Entomologist, 41(2): 67-71.

Distribuicão no Brasil: SC (Nova Teutônia)

Hábitat: gramíneas

\section{Gênero Heliothrips}

Heliothrips haemorrhoidalis (Bouché, 1833: 42) (Thrips) (Europa: Berlin, Alemanha) (casa-de-vegetação) Bouché, P.F. 1933. Naturgeschichte der schädlichen und nützlichen Garte-Insekten und die bewähtesten Mittel zur Vertilgung der ersteren. Berlin. pp. 42-45.

Distribuicãa no Brasil: AM (Manaus), BA, MG (Carmo do Rio Claro), MS (Selvíria), PA, PE (Petrolina), RJ (Jardim Botânico, Jacarepaguá/Rio de Janeiro), RS, SP (Vargem Grande do Sul)

Hábitat: inflorescências de limão (Citrus sp.); unindentified hedge plant; numerosas plantas; macadâmia; folhas de videira; folhas de seringueira; folhas jovens de guaraná (Paullinia cupana)

Heliothrips zucchi Mound \& Monteiro, 1997: 156 (Brasil: Mata de Santa Genebra, Campinas, SP) (folhas de ?Meliaceae)

Mound, L.A. \& Monteiro, R.C. 1997. A review of the genus Heliothrips (Thysanoptera; Thripidae), with a new sister-species of the greenhouse thrips from South Eastern Brazil). Journal of the New York Entomological Society, 105(3-4): 154-160.

Distribuicão no Brasil: SP (Campinas, Piracicaba), RJ (Jacarepaguá/Rio de Janeiro)

Hábitat: folhas de ?Meliaceae; folhas de arbustos; ramos mortos

\section{Gênero Hercinothrips}

Hercinothrips bicinctus (Bagnall, 1919: 258) (Heliothrips) (Bélgica: Bruxelas)

Bagnall, R.S. 1919. Brief descriptions of new Thysanoptera. X. Annals and Magazine of Natural History, $9^{\text {th }}$ Series, 4(22): 253-277.

Distribuicão no Brasil: SP

Hábitat: rainha-da-noite

Hercinothrips femoralis (Reuter, 1891: 166) (Heliothrips) (Filândia: Helsinki)

Reuter, O.M. 1891. Thysanoptera, funna I finska orangerier. Meddelelser of Societas pro Fauna et Flora Fennica, 17: 160-167.

Sinônimos: Heliothrips apicalis Bondar, 1931: 86 (Brasil: BA) (Hiptis sp.)

Bondar, G. 1931. Un novo genero e tres novas especies de Thysanopteros Heliothripineos, encontrados na Bahia. Archivos do Instituto Biológico, São Paulo, 4: 83-88.

Distribuição no Brasil: BA

Hábitat: Hiptis sp. 


\section{Gênero Hoodothrips}

Hoodothrips constrictus (Hood, 1925: 51) (Astrothrips) (Trinidade) (Lantana sp.)

Hood, J.D. 1925. New neotropical Thysanoptera collected by C.B. Williams. Psyche, 32(1): 48-69.

Sinônimos:

Hoodothrips neivai Bondar, 1931: 84 (Brasil: BA) (Apocynaceae)

Bondar, G. 1931. Um novo genero e tres novas especies de Thysanopteros Heliothripineos, encontrados na Bahia. Arquivos do Instituto Biológico, 4: 83-88.

Tryphactothrips ipomoeae Moulton, 1933: 98 (Brasil: ? BA) (Ipomoea batatas)

Moulton, D. 1933. The Thysanoptera of South America. II. Revista de Entomologia, 3(1): 96-133.

Distribuicãa no Brasil: ?BA; SP (Piracicaba)

Hábitat: Ipomoea batatas; folhas de Dahlia variabilis; folhas de uma Apocynaceae; Acucena sp.; folhas e flores de pimentinha; folhas de Galinsoga ciliata (daninha botão de ouro)

Hoodothrips lineatus (Hood, 1927: 242) (Tryphactothrips) (Trinidade: Turure River) (planta não determinada) Hood, J.D. 1927. New Neotropical Thysanoptera collected by C.B. Williams. II. Psyche, 34(6): 230-246.

Sinônimo: Hoodothripoides braziliensis Mound, 1970: 54 (Brasil: Nova Teutônia, SC) (desconhecido)

Mound, L.A 1970. Studies on heliothripine Thysanoptera. Proceedings of the Royal Entomological Soceity of London, Series B (Taxonomy), 39(3-4): 41-56 + plate I.

Distribuicão no Brasil: SC (Nova Teutônia)

Hábitat: ramos secos; trepadeiras; Terminalia sp.

\section{Hoodothrips sp.}

Distribuição no Brasil: SP (Mata de Santa Genebra/Campinas)

Hábitat: Hepathorium maximilinae; citros

\section{Gênero Oneilliella}

Oneilliella williamsi (Hood, 1927: 239) (Hercothrips) (Trinidade: St. Annes) (várias plantas: coletando com rede)

Hood, J.D. 1927. New Neotropical Thysanoptera collected by C.B. Williams. II. Psyche, 34(6): 230-246.

Distribuicão no Brasil: SP (Mata de Santa Genebra/Campinas)

Hábitat: bambu; várias plantas (coletando com batidas)

Oneilliella sp. $\mathbf{n}$.

Distribuição no Brasil: SC (Nova Teutônia)

Habitat: serrapilheira

\section{Gênero Retithrips}

Retithrips syriacus (Mayet, 1890: 451) (Thrips (Heliothrips)) (Síria: Jaffa) (sem dado)

Mayet, V. 1890. Les insects de la vigne. Bibliotheque Du Progress Agricole Et Viticole: 448-453.

Sinônimos:

Stylothrips bondari Bondar, 1924: 46 (Brasil: BA) (roseira)

Bondar, G. 1924. Pragas das roseiras na Bahia (Stylothrips bondari Morgan). Correio Agrícola, 2(2): 46-47.

Retithrips aegyptiacus Marchal, 1910: 17 (Egito: Cairo)

Marchal, P. 1910. Sur un nouveau "Thrips", vivant sur la vigne en Egypte. Bulletin de la Société Entomologique d'Egypt, 1: 17-20

Distribuicão no Brasil: BA (Inharn Bupé), MG (João Pinheiro), PA, PE (Petrolina), RJ, SP (Piracicaba)

Hábitat: rosas; folhas maduras (parte inferior da copa) de eucalipto (Eucalyptus sp.); folhas de videira; galho seco de uma árvore 


\title{
Gênero Selenothrips
}

Selenothrips rubrocinctus (Giard, 1901: 263) (Physopus) (Guadalupe: Saint Calude) (folhas de cacau) Giard, A. 1901. Sur un thrips (Physopus rubrocincta nov. sp.) nuisible au Cacaoyer. Bulletin de la Société Entomologique de France, 15: 263-265.

Distribuição no Brasil: AM (Manaus), BA, MG, MS (Três Lagoas), PA, PE (Olinda, Petrolina), RJ, RS, SP (Piracicaba, Vargem Grande do Sul)

Hábitat: folhas de cacau (Theobroma cacao); folhas de mangueira; folhas de eucalipto (Eucalyptus urophylla); jambo branco (Eugenia aquea -Myrtaceae); folhas de mangostão (Garcinia mangostana); galho seco de árvore; macadâmia

\section{THRIPIDAE - THRIPINAE}

\author{
Gênero Anaphothrips
}

\section{Anaphothrips sp.}

Distribuição no Brasil: SP (Jaboticabal, Juquiá)

Hábitat: Chamaesyce hirta; Dracena marginata colorama

\section{Gênero Aneristothrips}

Aneristothrips (sic.) claripennis (Moulton, 1933: 229) (Mycterothrips) (Brasil: Santa Thereza, ES) (Achyroclime satureoides)

Moulton, D. 1933. The Thysanoptera of South America III. Revista de Entomologia, 3(2): 227-262

Distribuicão no Brasil: ES (Santa Thereza)

Hábitat: Achyroclime satureoides

\section{Gênero Arorathrips}

Arorathrips fulvus (Moulton, 1936: 182) (Chirothrips) (Havai: Paumalu, Oahu) (verificar hospedeiro na revista)

Moulton, D. 1936. Thysanoptera of the Hawaiiann Islands. Proceedings of the Hawaiian Entomological Society, 9(2): 181-188.

Sinônimo:

Chirothrips dolosus De Santis, 1967: 5 (Brasil: Pelotas, RS) (hábitat desconhecido)

De Santis, L. 1967. Dos nuevas especies de Tisanópteros del Brasil. Notas Comisión de Investigación Cientifica, Provincia de Buenos Aires, 4(6): 3-6.

Distribuicão no Brasil: RS, SP

Hábitat:

Arorathrips mexicanus (Crawford, 1909: 114) (Chirothrips) (México: Guadalajara) (flores de fumo Nicotiana tabacum)

Crawford, D.L. 1909. Some Thysanoptera of Mexico and the south, I. Pomona College Joumal of Entomology, 1(4): 109-119.

Distribuicãa no Brasil: MG, SP (Jaboticabal, Piracicaba)

Hábitat: inflorescência e folhs de gramineas, entre elas pé-de-galinha (Eleusine indica); seeds heads de Chloris guayana, folhas de cenoura

Arorathrips nigriceps (Hood, 1952: 147) (Chirothrips)(Brasil: Nova Teutônia, SC) (Dalbergia sp.)

Hood, J.D. 1952. Brasilian Thysanoptera III. Proceedings of the Biological Society of Washington, 65: 141-174. 
Distribuição no Brasil: SC (Nova Teutônia)

Hábitat: Dalbergia sp.

Arorathrips spiniceps (Hood, 1915: 12) (Chirothrips)

Hood, J.D. 1915. Descriptions of new American Thysanoptera. Insecutor Inscitiae Menstruus, 3: 1-40.

Distribuição no Brasil: SP (Piracicaba)

Hábitat: base de gramíneas

Arorathrips xanthius (Hood, 1933: 408) (Chirothrips) (Panamá: Barro Colorado Island/Zona do Canal) (ramo morto)

Hood, J.D. 1933. New thysanoptera from Panama. Joumal of the New York Entomological Society, 41(4): 407-434.

Distribuição no Brasil: SP (Jaboticabal)

Hábitat: gramíneas, entre elas Brachyaria plantaginea

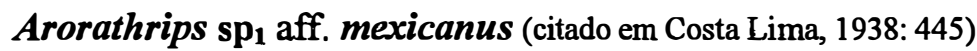

Costa Lima, A.M. da. 1938. Ordem Thysanoptera. In: Insetos do Brasil. Rio de Janeiro, ENA, 1938. tomo 1, n.2: 405-452.

Distribuição no Brasil:

Hábitat:

Arorathrips $\mathbf{s p}_{2}$.

Distribuicãa no Brasil: SP (Piracicaba)

Hábitat: folhas de gramineas

\section{Gênero Aurantothrips}

Aurantothrips orchidearum (Bondar, 1931:435) (Anaphothrips) (Brasil: BA)

Bondar, G. 1931. Uma nova praga das orquídeas (Anaphothripsorchidearum Bondar) e meio de combater este e outros thysanopterros damninhos. Chácaras e Quintaes, 44: 435: 436.

Distribuição no Brasil: BA, MG, RJ, SP (Piracicaba, Santos)

Hábitat: flores cor-de-rosa de Cattleya autumnalis; flores amarelas de Laelia glandis; Coryanthes sp.; Cyrtopodium sp.; Catasetum sp.; Miltonia sp.

\section{Gênero Bradinothrips}

Bradinothrips musae (Hood, 1956: 216) (Palleucothrips) (Equador: Quevedo) (banana)

Hood, J.D. 1956. Two new Thripidae (Thysanoptera) from banana. Proceedings of the Biological Society of Washington, 69: 215-218.

Distribuicão no Brasil: SP (Registro, Sete Barras)

Hábitat: frutos de banana (Musa sp.)

\section{Gênero Bregmatothrips}

Bregmatothrips venustus Hood, 1912: 67 (USA: Brownsville, Texas) (não mencionado)

Hood, J.D. 1912. New genera and species of North American Thysanoptera from the South and West. Proceedings of the Biological Society of Washington, 25: 61-76.

Distribuicão no Brasil: SP (Jaboticabal, Piracicaba)

Hábitat: folhas e flores de graminea (Eleusine indica) e outras daninhas 


\section{Gênero "Ceratothripoides"}

“Ceratothripoides"sensu lato lagoenacollus (Moulton, 1933: 130) (Taeneothrips) (Brasil: Santa Thereza, ES) (desconhecido)

Moulton, D. 1933. The Thysanoptera of South America II. Revista de Entomologia, 3(1): 96-133.

Distribuicão no Brasil: ES (Santa Thereza)

Hábitat: desconhecido; Casearia sylvestris

\section{Gênero Cercyothrips}

Cercyothrips striatus Morgan, 1925: 1 (Porto Rico: Rio Piedras) (arbusto trepadeiro ou trepadeira)

Morgan, A.C. 1925. A new genus, a new subgenus and seven new species of Thysanoptera from Porto Rico. Florida Entomologist, 9(1): 1-7.

Distribuicão no Brasil: sul do Brasil

Hábitat: arbusto trepadeiro ou cipó

\section{Gênero Chaetanaphothrips}

Chaetanaphothrips orchidii (Moulton, 1907: 43, 52) (Euthrips) (USA: Fruitvale, Alameda County, Califórnia)

Moulton, D. 1907. A contribution to our lnowledge of the Thysanoptera of California. Technical series, USDA

Bureau of Entomology, 12: 39-68.

Distribuicão no Brasil: SP (Mogi-Mirim)

Hábitat: frutos de citros

\section{Gênero Chaetisothrips}

Chaetisothrips sp. aff. Chaetisothrips striatus (Hood, 1935: 166) (Isochaetothrips) (Panamá: Barro Colorado Island, Canal da Zona) (flores de Coutarea hexandra)

Hood, J.D. 1935. Journal of the New York Entomological Society, 43(2): 143-171 (pranchas com figuras nas páginas 169 e 171)

Distribuicão no Brasil: RJ, RS, SP (Piracicaba)

Hábitat: flores cor-de-rosa de Coutarea hexandra (Asteraceae)

\section{Gênero Charassothrips}

Charassothrips incomparabilis (Johansen, 1983: 104) (Humboldthrips)

Johansen, R. M. 1983. Nuevas trips (Insecta: Thysanoptera; Terebrantia, Thripidae, Thripinae) de La Sierra Madre Oriental y del eje Volcánico Transversal de México. Anales del Instituto de Biologia. Universidad Nacional Autonoma de México, 53: 91-132.

Distribuicão no Brasil: SP (Piracicaba)

Hábitat: inflorescências de Piper spp.

Charassothrips urospathae Hood, 1954: 200 (Brasil: Belém, PA)(flores de Urospatha caudata)

Hood, J.D. 1954. Brasilian Thysanoptera V. Proceedings of the Biological Society of Washington, 67: 195-214.

Distribuição no Brasil: PA (Belém)

Hábitat: flores de Urospatha caudata 


\section{Gênero Chirothrips}

Chirothrips priesneri Hood, 1949: 14 (Brasil: Fazenda Salto, São Carlos, SP) (Andropogon sp. provavelmente condensatus)

Hood, J.D. 1949. Brasilian Thysanoptera I. Revista de Entomologia, 20(1-3): 3-88.

Distribuição no Brasil: SP (Serra da Cantateira/Franco da Rocha, São Carlos)

Hábitat: gramíneas, incluindo Andropogon sp., provavelmente $A$. condensatus

"Chirothrips" sensu lato pubescens (Hood, 1949: 20) (Brasil: Serra da Cantareira, Franco da Rocha, SP)

Hood, J.D. 1949. Brasilian Thysanoptera I. Revista de Entomologia, 20(1-3): 3-88.

Distribuicão no Brasil: SP (Franco da Rocha)

Hábitat: gramíneas

“Chirothrips” sensu lato sericatus (Hood, 1949: 18) (Brasil: Jacarepaguá, Rio de Janeiro, RJ)

Hood, J.D. 1949. Brasilian Thysanoptera I. Revista de Entomologia, 20(1-3): 3-88.

Distribuição no Brasil: RJ (Jacarepaguá/Rio de Janeiro, Rio de Janeiro), SP

Hábitat: Andropogon condensatus

“Chirothrips” sensu lato texanus Andre, 1939: 200 (USA: Denison, Texas) (trevo: coletando com rede)

Andre, F. 1939. A synopsis of the American species of Chirothrips Haliday (Thysanoptera). Proceedings of the Entomological Society of Washington, 41(6): 192-204.

Distribuicão no Brasil: SP (Serra da Cantareira)

Hábitat: várias gramíneas; trevo

\section{Gênero Coremothrips}

Coremothrips nubilicus (Hood, 1954: 213) (Brasil: Nova Teutônia, SC) (Dalbergia sp.)

Hood, J.D. 1954. Brasilian Thysanoptera V. Proceedings of the Biological Society of Washington, 67: 195-214.

Distribuicão no Brasil: SC (Nova Teutônia)

Hábitat: Dalbergia sp.

Coremothrips pallidus Hood, 1925: 52 (Trinidade: Evasdale, próximo a Sangre Grande) (em folhas de cacau, abacate e pera)

Hood, J.D. 1925. New Neotropical Thysanoptera collected by C.B. Williams. Psyche, 32(1): 48-69.

Distribuicão no Brasil: SP (Piracicaba)

Hábitat: folhas de Trema micrantha

\section{Gênero Corynothrips}

Corynothrips flavus Moulton, 1941: 314 (Brasil: MG) (Leguminosa "Fedegosa")

Moulton, D. 1941. Thysanoptera from Mingas Geraes, Brazil (second paper). Revista de Entomologia, 12(1-2): 314322.

Distribuicão no Brasil: MG

Hábitat: Leguminosa "Fedegosa"

Corynothrips stenopterus Williams, 1913: 210 (West Índia: Kingstown, St. Vicent) (Manihot utilissima) Williams, C.B. 1913. On two new species of Thysanoptera from the West Indies. Journal of Economic Biology, 8(4): 209-215.

Distribuicão no Brasil: SP

Hábitat: folhas de mandioca (Manihot utilissima) 


\section{Gênero Dendrothripoides}

Dendrothripoides innoxius (Kamy, 1914: 359) (Euthrips) (Indonésia: Semarang)

Kamy, H. 1914. Beiträge zur Kenntnis der Gallen von Java. Zweite Mitteilung über die javanischen Thysanopterocecidien und deren Bewohner. Zeitschrift für wissenschaftliche Insektenbiologie, 10(8-9): 355-369. Sinônimos:

Dendrothripoides ipomeae Bagnall, 1923: 625 (Índia: Maddur, Mysore) (Ipomoea staphylina)

Bagnall, R.S. 1923. Brief descriptions of new Thysanoptera. XIV. Annals and Magazine of Natural History, $9^{\text {th }}$ Series, 12: 624-631.

Heliothrips ipomeae Bondar, 1930: 345 (Brasil: BA) (Ipomoea sp.)

Bondar, G. 1930. Insectos damninhos e molestias da batata doce no Brasil. Correio Agrícola, 8(12): 343-348.

Distribuicão no Brasil: BA

Hábitat: batata doce; Ipomoea sp.

\section{Gênero Dorcadothrips}

Dorcadothrips xanthius (Williams, 1917: 59) (Physothrips) (Trinidade: Port of Spain) (orquideas Cattleya sp.) Williams, C.B. 1917. A new thrips damaging orchids in the West Indies. Bulletin of Entomological Research, 8(1): 59-61.

Distribuição no Brasil: SP

Hábitat: folhas de orquideas Cattleya labiata, Laelia purpurata e diversos híbridos de Cattleya

\section{Gênero Echinothrips}

Echinothrips asperatus Hood, 1957: 130 (Brasil: Nova Teutônia, SC) (folhas caidas)

Hood, J.D. 1957. New Brazilian Thysanoptera. Proceedings of the Biological Society of Washington, 70: 129-180.

Distribuição no Brasil: SC (Nova Teutônia), SP (Mata de Santa Genebra/Campinas)

Hábitat: folhas caídas; Ibantus sp.

Echinothrips mexicanus Moulton, 1911: 37 (México: Acapulco)

Moulton, D. 1911. Synopsis, catalogue and bibliography of North American Thysanoptera, with descriptions of new species. Technical Series, USDA Bureau of Entomology, 21: 1-56.

Distribuicão no Brasil: BA, SP (Campinas, Piracicaba)

Hábitat: Eucalyptus citriodora; abobrinha (Cucurbita pepo cv. Caserta), soja

Echinothrips pinnatus Hood, 1960: 57 (Brasil: Nova Teutônia, SC) (serrapilheira de floresta primária)

Hood, J.D. 1960. Six new Thysanoptera from Brazil. Revista Brasileira de Entomologia, 9 (1-4): 57-68.

Distribuição no Brasil: SC (Nova Teutônia)

Hábitat: serrapilheira de floresta primária

Echinothrips n. sp. (Brasil)

Distribuição no Brasil: MG (Sete Lagoas), PR, SP (Araras, Campinas)

Hábitat: soja; milho

\section{Gênero Enneothrips}

Enneothrips (Enneothripiella) flavens Moulton, 1941: 318 (Brasil: MG) (chá-da-Índia)

Moulton, D. 1941. Thysanoptera from Minas Geraes, Brazil (second paper). Revista de Entomologia, 12: 314-322.

Distribuição no Brasil: MG, SP (Campinas, Itápolis, Jaboticabal, Piracicaba, Tupi)

Hábitat: chá da Índia; amendoim (Arachis hypogaea) 
Enneothrips (Enneothripiella) fuscus Hood, 1954: 209 (Brasil: Corcovado, tha de Marajó, próximo à Breves, PA) (ramos mortos com folhas)

Hood, J.D. 1954. Brasilian Thysanoptera V. Proceedings of the Biological Society of Washington, 67: 195-214.

Distribuicão no Brasil: PA

Hábitat: ramos mortos com folhas

\section{Gênero Frankliniella}

Frankliniella annulipes Hood, 1915: 16 (Panamá: Bouquete) (flores de um arbusto comum, com folhas brancas grandes)

Hood, J.D. 1915. Description of new American Thysanoptera. Insecutor Insicitiae Menstruus, 3(1-4): 1-40.

Distribuicão no Brasil: SP (orquidário do Depto. de Genética/ESALQ/Piracicaba)

Hábitat: flores rosa de Cattleya autumnalis e amarelas de Laelia glandis

Frankliniella ?australis Morgan, 1925: 139 (Argentina: Villa Vincencio, prov. de Mendoza) (inflorescência de Trichocereus candicans, Cactaceae)

Morgan, A.C. 1925. Six new species of Frankliniella and a key to the American species. Canadian Entomologist, 57(6): 136-147.

Distribuicão no Brasil: PR (Guaira)

Hábitat: flores de uma Compositae

Frankliniella bertelsi (De Santis, 1967: 2) (Isochaetothrips) (Brasil: Pelotas, RS) (?)

De Santis, L. 1967. Una nueva especie de Isochaetothrips del Brasil (Thysanoptera: Thripidae). Revista de la Museo de La Plata n.s., Zoologia, 10(72): 1-3.

Distribuicão no Brasil: RS (Pelotas), SC (Nova Teutônia)

Hábitat: Morus nigra, Sambucus niger, Sapium sp., desconhecido/coletando com rede

Frankliniella bicolor Moulton, 1948: 105 (Brasil: MG) (Buddleyha variabilis)

Moulton, D. 1948. The genus Frankliniella Kamy, with keys for the determination of species (Thysanoptera).

Revista de Entomologia, 19(1-2): 55-114.

Distribuicão no Brasil: MG

Hábitat: Buddleyha variabilis

Frankliniella bondari Hood, 1941: 622 (Brasil: BA) (Polyanthes tuberosa)

Hood, J.D. 1941. A century of new American Thysanoptera III. Revista de Entomologia, 12(3): 547-678.

Distribuicão no Brasil: BA

Hábitat: Polyanthes tuberosa

Frankliniella brevicaulis Hood, 1937: 113 (Panamá: Juan Diaz) (arbusto?)

Hood, J.D. 1937. Studies on Neotropical Thysanoptera III. Revista de Entomologia, 7(1): 96-115.

Distribuicão no Brasil: ES, SP (Pariquera, Piracicaba, Sete Barras)

Hábitat: inflorescência e frutos de banana (Musa sp.), danificando os frutos; flores cor-de-rosa de Coutarea hexandra (Asteraceae); flores de quaresmeira (Tibouchina sp)

Frankliniella brunnea Priesner, 1932: 174 (México: Mexiko-Stadt)(Tithonia tubiformis)

Priesner, H. 1932. Neue Thysanopteren aus Mexiko, gesammelt von Prof. Dr. A. dampf. Teil 1. Wiener

Entomologischer Zeitung, 49: 170-185.

Sinônimo: Frankliniella tridacana Hood, 1937: 104 (Barro Colorado Island, Zona do Canal) (flores de Tridax procumbens)

Hood, J.D. 1937. Studies in Neotropical Thysanoptera III. Revista de Entomologia, 7(1): 96-115.

Distribuicão no Brasil: SP (Paranapanema)

Hábitat: flor de crisântemo 
Frankliniella caseariae Moulton, 1933: 111 (Brasil: Colônia Rio Bonito, Santa Leopoldina, ES) (Casearia sylvestris)

Moulton, D. 1933. The Thysanoptera of South America II. Revista de Entomologia, 3(1): 96-133.

Distribuicão no Brasil: ES (Colônia Rio Bonito/Santa Leopoldina)

Hábitat: Casearia sylvestris

Frankliniella condei John, 1927: 152 (Brasil: Fazenda Dumont, Colônia Santa Amélia, Ribeirão Preto, SP) (Bromeliaceae)

John, O. 1927. A new Frankliniella (Thysanoptera) from Brazil. Bulletin \& Annals de la Societé Entomologique de Belgique, 68(8): 152-154.

Sinônimo: Frankliniella condei f. albicans John, 1928: 154 (Brasil: Ribeirão Preto, SP)

Distribuição no Brasil: MG, SP (Holambra, Paranapanema, Ribeirão Preto, Taquaritinga)

Hábitat: Bromeliaceae; abacate; flores de roseira; folhas de chá-da-Índia; Citrus sp.; bromélia; inflorescências de manga, de laranja, de "murcot" e de limão; "arbusto-do-mato"; crisântemo

Frankliniella desantisi Sakimura \& O'Neill, 1979: 17 (Brasil: Nova Teutônia, SC) (coletando com rede)

Sakimura, K. \& K. O'Neill. 1979. Frankliniella, redefinition of genus and revision of the minuta group species (Thysanoptera: Thripidae). USDA Technical Bulletin 1572, 49pp.

Distribuição no Brasil: SC (Nova Teutônia)

Hábitat: desconhecido (coletando com rede); Myrtaceae

Frankliniella difficilis Hood, 1925: 73 (Martinica e Guadalupe) (várias flores/coletando com rede)

Hood, J.D. 1925. New species of Frankliniella (Thysanoptera). Bulletin of the Brooklyn Entomological Society, 20(2): 71-83.

Distribuição no Brasil: SP (Limeira), RJ (Guanabara/Rio de Janeiro)

Hábitat: flores de Poncirus trifoliata

Frankliniella distinguenda Bagnall, 1919: 264 (Paraguai: Puerto Max) (inflorescência de Vemonia sp.)

Bagnall, R.S. 1919. Brief descriptions of new Thysanoptera. X. Annals and Magazine of Natural History, $9^{\text {th }}$ Series, 4(22): 253-277.

Sinônimo:

Isochaetothrips varicomis Moulton, 1933: 127 (Brasil: Santa Thereza, ES) (Verbesina glabrata)

Moulton, D. 1933. The Thysanoptera of South America II. Revista de Entomologia, 3(1): 96-133.

Frankliniella verbesinae Sakimura, 1967: 168 (Brasil: Santa Thereza, ES) (Verbesina glabrata)

Sakimura, K. 1967. A preliminary note on a review of the genus Chaetisothrips Priesner (Thysanoptera: Thripidae).

Florida Entomologist, 50: 165-168.

Frankliniella vernoniae Priesner, 1921: 190 (Paraguai)

Priesner, H. 1921. Neue und wenig bekannte Thysanopteren der neotropischen Fauna aus der Sarnmlung des

Berliner Zoologischen Museums. Deutsche Entomologische Zeitschrift, 1921(3): 187-223.

Distribuição no Brasil: RJ, SP (Holambra, Paranapanema)

Hábitat: Verbesina glabrata; inflorescências de limão; inflorescências de laranja; Eugenia jaboticabae; rosa; desconhecido (no RJ)

Ref.: Moulton, D. 1933, 3(1) (todas hosp., exceto V. glabrata)

Frankliniella fulvipennis Moulton, 1933: 121 (Brasil: São Paulo, SP) (“arbusto-do-mato")

Moulton, D. 1933. The Thysanoptera of South America II. Revista de Entomologia, 3(1): 96-133.

Distribuição no Brasil: ES (Colônia Rio Bonito/Santa Leopoldina), SP (São Paulo)

Hábitat: “arbusto-do-mato"; flores de Leguminosa minosoidea; Vernonia polyanthes

Frankliniella fulvipes Bagnall, 1919: 265 (Argentina: Tucumán) (Solanum sp.?)

Bagnall, R.S. 1919. Brief descriptions of new Thysanoptera. X. Annals and Magazine of Natural History, $9^{\text {th }}$ Series, 4(22): 253-277.

Sinônimo: Frankliniella sulfuripes Hood, 1937: 98 (Panamá)

Distribuição no Brasil: MG, PR (Rio Azul), SC 
Hábitat: Solanum sp.; Acalypha sp.; várias plantas especialmente Solanaceae; flor de furno bravo

Frankliniella fusca (Hinds, 1902: 148, 154) (Euthrips) (USA: Massachusetts) (confirmar hospedeiro?)

Hinds, W.E. 1902. Contribution to a monograph of the insects of the order Thysanoptera inhabiting North America.

Proceedings of the United States National Museum, 26: 79-242.

Distribuicão no Brasil: SP

Hábitat: amendoim

Frankliniella fuscicornis Moulton, 1948: 94 (substituição do nome fulvicomis) (Brasil: São Paulo, SP)

(flores de limoeiro)

Moulton, D. 1948. The genus Frankliniella Kamy, with keys for the determination os species (Thysanoptera).

Revista de Entomologia, 19: 55-114.

Sinônimo: Frankliniella fulvicornis Moulton, 1933: 124 nec Uzel (Brasil: SP) (inflorescência de limão)

Moulton, D. 1933. The Thysanoptera of South America II. Revista de Entomologia, 3: 96-133.

Distribuicão no Brasil: SP (São Paulo)

Hábitat: flores de limoeiro

Frankliniella gardenia Moulton, 1948: 107 (México) (gardênia)

Moulton, D. 1948. The genus Frankliniella Karny, with keys for the determination os species (Thysanoptera).

Revista de Entomologia, 19: 55-114.

Distribuicãa no Brasil: SP (Mogi-Minim, Taquaritinga)

Hábitat: flores de laranja e de "murcot"; gardênia

Frankliniella gemina Bagnall, 1919: 265 (Paraguai: Puerto Max)

Bagnall, R.S. 1919. Brief descriptions of new Thysanoptera. X. Annals and Magazine of Natural History, $9^{\text {th }}$ Series, 4(22): 253-277.

Sinônimo: Frankliniella rodeos var. allochroos Moulton, 1933: 116 (Brasil: SP) (inflorescências de laranja e limão)

Moulton, D. 1933. The Thysanoptera of South America II. Revista de Entomologia, 3(1): 96-133.

Distribuicão no Brasil: PR (Guaíra), RJ, RS, SP

Hábitat: inflorescências de laranja e limão; jurubeba; Leguminosa mimosiodea; rosa; Ambrosia polystachya (planta daninha); flores de uma Compositae

Frankliniella hemerocallis Crawford, 1948: 83 (EUA: Lake Geneva, Wisconsin) (flores de Hemerocallis sp.) Crawford, J.C. 1948. A new species of Frankliniella on Hemerocallis (Thysanoptera, Thripidae). Proceedings of the Entomological Society of Washington, 50(4): 83-84.

Distribuição no Brasil: SP (Jaboticabal)

Hábitat: guizo-de-cascavel (Crotalaria incana)

Frankliniella insularis (Franklin, 1908: 715) (Euthrips) (Barbados) (flores de numerosas plantas?)

Franklin, H.F. 1908. On a collection of Thysanopterous insects from Barbados and St. Vicent Islands. Proceedings of the U.S. Nacional Museum, 33: 715-730.

Distribuição no Brasil: BA, ES (Colônia Rio Bonito/Santa Leopoldina), MG (Sete Lagoas), RJ (Estação Biológica do Jardim Botânico/Monte Itatyaia), RS, SC, SP (Holambra, Campinas, Limeira, Piracicaba)

Hábitat: Chrysanthemum sp.; feijão-guandu (Cajanus cajan); fumo; mangalô; Passiflora sp.; trapoeiraba; laranjeira; milho; flores vermelhas de Malvaviscus sp. (arbusto de jardim); Poncinus trifoliata; Rosa sp.; Bignoniacea; Cyperus surinamensis; flores e frutos de acerola; flores de quaresmeira (Tibouchina sp)

Frankliniella konoi Sakimura \& O'Neill, 1979: 22 (Brasil: Nova Teutônia, SC) (ramos mortos)

Sakimura, K \& K O'Neill. 1979. Frankliniella, redefinition of genus and revision of the minuta group species (Thysanoptera: Thripidae). USDA Technical Bulletin 1572, 49p.

Distribuicão no Brasil: SC (Nova Teutônia)

Hábitat: ramos mortos 
Frankliniella longipennis (Moulton, 1933: 126) (Exophthalmothrips) (Brasil: Santa Thereza, ES) (desconhecido/coletando com rede)

Moulton, D. 1933. The Thysanoptera of South America II. Revista de Entomologia, 3(1): 96-133.

Sinônimo: Exophthalmothrips moultoni Hood, 1941: 595 (Peru: Almirante, Dept. Amazonas) (flores de Fuchsia decussata)

Hood, J.D. 1941. A century of new American Thysanoptera III. Revista de Entomologia, 12(3): 547-678.

Distribuicão no Brasil: ES (Santa Thereza), RJ (Estação Biológica do Jardim botânico/Monte Itatiaya)

Hábitat: desconhecido

Frankliniella longispinosa Moulton, 1933: 113, 116 (Brasil: Santa Thereza, ES) (desconhecido)

Moulton, D. 1933. The Thysanoptera of South America II. Revista de Entomologia, 3: 96-133.

Distribuicão no Brasil: ES (Santa Thereza), MG

Hábitat: uma trepadeira

Frankliniella minor Moulton, 1948: 87 (revised status) (Brasil) (folhagem de citros)

Moulton, D. 1948. The genus Frankliniella Kamy, with keys for the determination os species (Thysanoptera).

Revista de Entomologia, 19(1-2): 55-114.

Sinônimo: Frankliniella. difficilis var. minor Moulton, 1948: 88

Moulton, D. 1948. The genus Frankliniella Kamy, with keys for the determination os species (Thysanoptera).

Revista de Entomologia, 19(1-2): 55-114.

Distribuição no Brasil: MG

Hábitat:

Frankliniella minuta (Moulton, 1907: 44, 56) (Euthrips) (USA: Berkeley, Califómia) (gramínea)

Moulton, D. 1907. A contribuition to our knowledge of the Thysanoptera of Califormia. United States Department of Agriculture, Bureau of Entomology Technical Series, 12: 39-68.

Distribuicão no Brasil: RS

Hábitat: em folhas de alfafa, melão, rosaceae, tomateiro, videira (pedúnculos florais) e outras plantas

Frankliniella nakaharai Sakimura \& O’Neill, 1979: 26 (Brasil: Nova Teutônia, SC) (não determinado)

Sakimura, K. \& K. O'Neill. 1979. Frankliniella, redefinition of genus and revision of the minuta group species (Thysanoptera: Thripidae). USDA Technical Bulletin 1572, 49pp.

Distribuição no Brasil: SC (Nova Teutônia)

Hábitat: não determinado; Morus nigra

Frankliniella occidentalis (Pergande, 1895: 392) (Euthrips) (folhas de damasco e inflorescência de laranja) Pergande, T. 1895. Observations on certain Thripidae. Insect Life, 7(5): 390-395.

Sinônimo: Euthrips tritici var. californicus Moulton, 1911: 28

Moulton, D. 1911. Synopsis, cataloque, and bibliography of North American Thysanoptera, with descriptions of new species. United States Department osf Agriculture, Bureau of Entomology Technical Series, 21: 1-56.

Distribuição no Brasil: AL (Chã de Pilar), PE (Camocim de São Felix), RJ (Cachoeiras de Macacu), RS (Vacaria), SP (Atibaia, Campinas, Holambra, Jaguariúna, Paranapanema, Santo Antonio de Posse)

Hábitat: flores e folhas de crisântemo; rosa; flores de violeta africana; flores cor-de-rosas e vermelhas de alstroemeria; flores de cravo; flores de girassol; folhas e frutos de pêssego; flores e frutos de pimentão; alfafa (?); feijão (?); melão (?); tomateiro (?); videira (?); outras plantas (?)

Frankliniella oxyura Bagnall, 1919: 267 (Paraguai: Department of Concepcion, Puerto Max) (não determinado)

Bagnall, R.S. 1919. Brief descriptions of new Thysanoptera. X. Annals and Magazine of Natural History, $9^{\text {th }}$ Series, 4(22): 253-277.

Distribuição no Brasil: ES (Colônia Rio Bonito/Santa Leopoldina, Santa Thereza,), MG, RJ (Itatiaia), SC (Nova Teutônia), PR (Guaíra) 
Hábitat: Ageratum conyzoides; flores de Agave sp.; violeta; rosa; Inga marginata; Verbesina glabrata; desconhecido (coletando com rede); Vernonia polyanthes; Uricacea (Compositae); flores de Compositae não determinada; flores não conhecidas; Eupatorium sp.; gramineas; flores de Compositae

Frankliniella peruviana Hood, 1937: 102 (Peru: vizinhança de Sani Beni, floresta tropical, região de Satipo, Dept. Yunin, 840m) (flores)

Hood, J.D. 1937. Studies on Neotropical Thysanoptera III. Revista de Entomologia, 7(1): 96-115.

Distribuicão no Brasil: SP (Piracicaba)

Hábitat: flores; folhas de gramíneas

Frankliniella rodeos Moulton, 1933: 113, 115 (Brasil: Santa Thereza; ES) (flores de várias plantas, incluindo Baccharis eleagnoides, Baccharis trinervis, Casearia sylvestris, Cyperus surinamensis, Sagittaria montevidensis, flores de laranja, limão e ameixeira, rosa selvagem, manga, jurubeba)

Moulton, D. 1933. The Thysanoptera of South America II. Revista de Entomologia, 3(1): 96-133.

Distribuicão no Brasil: ES (Santa Thereza), DF (Brasília), MG, RJ, RS, SP (Piracicaba)

Hábitat: flores de Piper sp.; abobrinha (Cucurbita pepo cv. Caserta)

Frankliniella schultzei (Trybom, 1910: 151) (Physopus)

Trybom, F. 1910. Physapoda, in Schultze, Zoologische und anthropologische Ergebnisse einer Forschungsreise im westlichen un zentralen Südafrika (1903-1905). Denkschriften Medizinisch-naturwissenschaftlichen Gesellschaft zu Jena, 16: 147-174.

Sinônimos: Frankliniella paucispinosa Moulton, 1933: 122 (Brasil: RJ) (ipoméia "moming glory")

Moulton, D. 1933. The Thysanoptera of South America II. Revista de Entomologia, 3(1): 96-133.

Distribuicão no Brasil: BA (Curaçã), GO (Capinópolis, Rio Verde e Santa Helena), MA (São Luís), MS (Angélica, Caarapó, Centralina, Dourados, Ponta Porã, Selvíria, Taquarussu e Vicentina), PE (Santa Maria da Boa Vista, Petrolina), PR (Alto Araguari, Assaí, Formosa do Oeste, Itaguajé, Juranda, Lobato, Londrina, Mercedes, Nova Santa Bárbara, Palotina, Rio Azul, Santa Cecília do Pavão, São José do Ivaí, São Sebastião da Amoreira e Vera Cruz do Oeste), RJ, RN (Mossoró), RS (Santa Maria, RS (Santa Cruz do Sul), SP (Araras, Artur Nogueira, Campinas, Iracemápolis, Iha Solteira, Jaboticabal, Mogi-Guaçu, Mogi-Mirim, Piracicaba, Paulínia), SC (Chapecó e Rio do Sul)

Hábitat: melancia (Citrullus vulgaris); folhas de algodão (Gossypium hirsutum); alface; melão; folha de girassol (Helianthus annuus); rosa; amendoim (Arachis hypogaea); soja (Glycine max); tomate (Lycopersicon esculentum); batata; flores de fumo; folhas de pepino; cebola; pimentão (Helianthus annuum); daninhas brássicas, incluindo mostarda (Sinapsis arvensis); Nicandra physaloides, flores de videira; nabo forrageiro; abobrinha; ipoméia ("moming glory")

Frankliniella serrata Moulton, 1933: 125 (Brasil: Santa Thereza, ES) (sem dado hospedeiro)

Moulton, D. 1933. The Thysanoptera of South America II. Revista de Entomologia, 3(1): 96-133.

Distribuicão no Brasil: ES (Rhono Claudio, Santa Thereza), RJ (Itatiaia), SC (Nova Teutônia)

Hábitat: desconhecido; Sagitaria montevidensis; Eupatorium sp.; Sambucus nigra

Frankliniella ?setipes Bagnall, 1919: 266 (Argentina: Tucumán) (desconhecido)

Bagnall, R.S. 1919. Brief descriptions of new Thysanoptera. X. Annals and Magazine of Natural History, $9^{\text {th }}$ Series, 4(22): 253-277.

Distribuicão no Brasil: SP (Santos)

Hábitat: desconhecido

Frankliniella simplex Priesner, 1924: 532 (México: Desierto de los Leones) (Senecio sp.)

Priesner, H. 1924. Neue Thysanopteren. Sitzungsberichte der Kaiserlichen Akademie der Wissenschaften, 133: 527542.

Distribuição no Brasil: SP (Piracicaba) 
Hábitat: flores de orquídea; Senecio sp.

Frankliniella speciosa Moulton, 1933: 117 (Brasil: Colônia Rio Bonito, Santa Leopoldina, ES) (Leguminosae) Moulton, D. 1933. The Thysanoptera of South America II. Revista de Entomologia, 3(1): 96-133.

Distribuicão no Brasil: ES (Colônia Rio Bonito/Santa Leopoldina)

Hábitat: leguminosa

Frankliniella trinidadensis Hood, 1941: 623 (Trinidade: St. Clair) (gramínea/coletando com rede) Hood, J.D. 1942. A century of new american Thysanoptera III. Revista de Entomologia, 12(3): 547-678.

Distribuicão no Brasil: SP (Piracicaba, São Pedro)

Hábitat: folhas de gramíneas

Frankliniella tritici (Fitch, 1855: 385) (Thrips) (EUA: Geneva, Wisconsin)

Fitch, A. 1855. The wheat thrips \& three-banded thrips. Gentleman, 6(24): 385-386.

Distribuicão no Brasil: RS

Hábitat: trigo

Frankliniella varipes Moulton, 1933:114, 118(Brasil: Santa Thereza, ES)(Inga marginata)

Moulton, D. 1933. The Thysanoptera of South America II. Revista de Entomologia, 3(1): 96-133.

Distribuição no Brasil: ES (Santa Thereza), RJ (Estação Biológica do Jardim Botânico/Monte Itatiaya), SP

Hábitat: Inga marginata; inflorescências de limão e laranja; planta desconhecida

Frankliniella williamsi Hood, 1915: 19 (EUA: Washington, D.C., Maryland) (? cascas de milho fresco)

Hood, J.D. 1915. Descriptions of new American Thysanoptera. Insecutor Inscitiae Menstruus, 3(1-4): 1-40.

Distribuição no Brasil: MG (Sete Lagoas), PR (Arapongas, Cascavel, Londrina), SC (Canoinhas), SP (Holambra?, Rafard)

Hábitat: folhas novas de milho (Zea mays); pimentão ( $F$. ?williamsi); algodão ( $F$. ?williamsi)

Frankliniella zucchini Nakahara \& Monteiro, 1999: 290 (Brasil: Piracicaba, SP) (abobrinha Cucurbita pepo cv. Caserta)

Nakahara, S. \& RC. Monteiro. 1999. Frankliniella zucchini (Thysanoptera; Thripidae), a new species and vector of tospovirus in Brazil. Proceedings of the Entomological Society of Washington, 101(2): 290-294.

Distribuicão no Brasil: SP (Piracicaba)

Hábitat: folhas e flores de abobrinha (Cucurbita pepo cv. Caserta)

Frankliniella sp. $\mathbf{n}_{1}$. (Brasil: Nova Teutônia, SC) (hospedeiro com nome ilegivel)

Distribuicão no Brasil: SC (Nova Teutônia)

Hábitat: hospedeiro com nome ilegivel; ramos mortos

Frankliniella n. sp. (Brasil)

Distribuicão no Brasil: SP (Piracicaba)

Hábitat: inserção das folhas de milho (Zea mays)

Observação: extremidade do abdome é escura e o restante do corpo é amarelo

Frankliniella sp.

Distribuicão no Brasil: SP (São Pedro)

Hábitat: folhas de gramineas

Frankliniella sp. $_{2}$.

Distribuição no Brasil: SP (Santos)

Hábitat: sem dado 
Frankliniella sp. $_{3}$ - grupo Frankliniella bruneri (Watson, 1926: 54) (Euthrips cephalica var. bruneri) Watson, J.R. 1926. Two new Thysanoptera from Florida (Thysanoptera). Florida Entomologist, 9: 53-55.

Distribuicão no Brasil: SP (Floramar?)

Hábitat: crisântemo

Frankliniella $\mathrm{sp}_{4 .}$ aff. a $\boldsymbol{F}$. gardenia

Distribuicão no Brasil: SP (Piracicaba)

Hábitat: flores cor-de-rosa de Coutarea hexandra (Asteracea)

\section{Gênero Hydatothrips}

Hydatothrips tricinctus (Hood, 1927: 231) (Sericothrips) (Martinica) (gramínea/coletando com rede) Hood, J.D. 1927. New tropical Thysanoptera collected by C.B. Williams II. Psyche, 34(6): 230-246.

Distribuição no Brasil: MG

Hábitat: gramíneas; Hydrangea sp.; rosa

Ref.: Moulton, D. 1941, 12(1-2)

\section{Gênero Leucothrips}

Leucothrips pictus Hood, 1952: 144 (Brasil: Nova Teutônia, SC) (planta não identificada)

Hood, J.D. 1952. Brasilian Thysanoptera III. Proceedings of the Biological Society of Washington, 65: 141-174.

Distribuição no Brasil: SC (Nova Teutônia)

Hábitat: planta não identificada

\section{Leucothrips sp.}

Distribuicão no Brasil: SP (Jaboticabal)

Hábitat: Parthenium hysterophorus

\section{Gênero Microcephalothrips}

Microcephalothrips abdominalis (Crawford, 1909: 157) (Thrips) (México: Guadalajara) (Várias Compositae, Solanum sp., Dacus sp. e outras)

Crawford, D.L. 1909. Thysanoptera of Mexico and the south, II. Pomona college Joumal of Entomology, 2(1): 153170.

Distribuicão no Brasil: PR (Curitiba), SP (Piracicaba)

Hábitat: cravo-de-defunto (Tagetes patula); flores de Compositae; flores de Ageratum sp.

\section{Gênero Neohydatothrips}

Neohydatothrips flavicollis (Hood, 1954: 204) (Sericothrips) (Brasil: Iha da Gipoia, próximo à Angra dos Reis, RJ) (ramos mortos e Eleusine indica)

Hood, J.D. 1954. Brasilian Thysanoptera V. Proceedings of the Biological Society of Washington, 67: 195-214.

Distribuição no Brasil: RJ (Illha da Gipoia/próximo à Angra dos Reis), SP (Piracicaba)

Hábitat: ramos mortos; Eleusine indica; soja

Neohydatothrips luculentus (Moulton, 1938: 376) (Sericothrips) (Brasil: Viçosa, MG) (Compositae desconhecida)

Moulton, D. 1938. Thysanoptera from Minas Geraes, Brazil. Revista de Entomologia, 9(3-4): 374-382.

Distribuição no Brasil: MG (Viçosa)

Hábitat: unknown shrub in woods; Compositae desconhecida 
Neohydatothrips portoricensis (Morgan, 1925: 3) (Sericothrips) (Porto Rico: Rio Pedros = ?Rio Piedras) (não mencionado)

Morgan, A.C. 1925. A new genus, a new subgenus and seven new species of Thysanoptera fdrom Porto Rico.

Florida Entomologist, 9(1): 1-7.

Sinônimos:

Neohydatothrips latereostriatus John, 1929: 34 (Brasil: Afonso Cláudio, ES) (coletando com rede)

John, O. 1919. A new species of Thysanoptera from Brazil, representing a new genus. Bulletin \& Annales de la Société Entomologique de Belgique, 69(2): 33-36.

Sericothrips portoricensis var. extremus Moulton, 1941: 316 (Brasil: MG) (folhagem de café)

Moulton, D. 1941. Thysanoptera from Minas Geraes, Brazil (second paper). Revista de Entomologia, 12(1-2): 314322.

Scirtothrips batatae Bondar, 1930: 347 (Brasil: BA) (Ipomoea batatas)

Bondar, G. 1930. Insectos damninhos e molestias da batata doce no Brasil. Correio Agricola, 8(12): 343-348.

Distribuicão no Brasil: BA, ES, MG, PR, SP (Campinas, Piracicaba)

Hábitat: Ipomoea batatas; folhagem de café; batata doce; desconhecido (bandejá d'água); folhas de uma trepadeira

Neohydatothrips ruginosus (Hood, 1954: 202) (Sericothrips) (Brasil: Itaguai, RJ) (uma trepadeira)

Hood, J.D. 1954. Brasilian Thysanoptera V. Proceedings of the Biological Society of Washington, 67: 195-214.

Distribuicão no Brasil: RJ (Itaguaí)

Hábitat: uma trepadeira

Neohydatothrips samayunkur (Kudô, 1995: 169) (Japão: Shizuioka) (Hydatothrips) (Tagetes erecta)

Kudô, I. 1995. A new species of Hydatothrips (Thysanoptera: Terebrantia: Thripidae) on marigold in Japan and the United States. Applied Entomology and Zoology, 30: 169-176.

Distribuição no Brasil: PR (Curitiba)

Hábitat: cravo-de-defunto (Tagetes patula); Tagetes spp. (Compositae)

Neohydatothrips signifer (Priesner, 1932: 172) (Sericothrips) (México: Chapingo) (Senecio salignus)

Priesner, H. 1932. Neue Thysanopteren aus Mexiko, gesammelt von Prof. Dr. A. dampf. Teil 1. Wiener Entomologische Zeitung, 49(3): 170-185.

Distribuicão no Brasil: MG, SP (Juquiá)

Hábitat: Compositae desconhecida; Senecio saligna; Dracena marginata colorama

Neohydatothrips sp $\mathbf{s p}_{\mathbf{\bullet}}$ (citado em Monteiro, R.C.; L.A. Mound \& R.A. Zucchi, 1996. Thrips species from three counties of the State of São Paulo. Anais da Sociedade Entomológica do Brasil, 25(2): 351-353)

Distribuição no Brasil: SP (Juquiá, Piracicaba)

Hábitat: desconhecida; Dracena marginata colorama

Neohydatothrips sp.

Distribuicão no Brasil: SP (Mata de Santa Genebra/Campinas)

Hábitat: flores de Celtia sp.

\section{Gênero Plesiothrips}

Plesiothrips amblycauda Hood, 1925: 53 (Trinidade) (folha de copo-de-leite)

Hood, J.D. 1925. New Neotropical Thysanoptera collected by C.B. Williams. Psyche, 32(1): 48-69.

Distribuicão no Brasil: MG

Hábitat: folhas de banana; folhagem de arroz

Plesiothrips frequens (Moulton, 1938: 377) (Thrips) (Brasil: Viçosa, MG) (desconhecido)

Moulton, D. 1938. Thysanoptera from Minas Geraes, Brazil. Revista de Entomologia, 9(3-4): 374-382.

Distribuicãa no Brasil: MG (Viçosa) 
Hábitat: desconhecido

Plesiothrips longicollis Hood, 1954: 18 (Brasil: Nova Teutônia, SC) (gramíneas)

Hood, J.D. 1954. Brasilian Thysanoptera IV. Proceedings of the Biological Society of Washington, 67: 17-54.

Distribuição no Brasil: SC (Nova Teutônia)

Hábitat: gramineas

Plesiothrips maculosus Hood, 1954: 212 (Brasil: Nova Teutônia, SC) (desconhecido)

Hood, J.D. 1954. Brasilian Thysanoptera V. Proceedings of the Biological Society of Washington, 67: 195-214.

Distribuição no Brasil: SC (Nova Teutônia)

Hábitat: desconhecido

Plesiothrips octarthrus Hood, 1925: 52 (Trinidade) (desconhecido)

Hood, J.D. 1925. New Neotropical Thysanoptera collected by C.B. Williams. Psyche, 32(1): 48-69.

Distribuicão no Brasil: MG

Hábitat: folhagem de arroz

Plesiothrips sculpticollis Hood, 1950: 1 (Brasil: São Carlos, SP) (gramineas)

Hood, J.D. 1950. Brasilian Thysanoptera II. Revista de Entomologia, 21(1-2): 1-113.

Distribuicão no Brasil: SP (São Carlos)

Hábitat: gramineas em floresta

Plesiothrips setiventris Hood, 1950: 3 (Brasil: Campo Grande, RJ) (gramineas misturadas em um pomar de laranja em uma colina)

Hood, J.D. 1925. Brasilian Thysanoptera I. Revista de Entomologia, 21(1-2): 1-113.

Distribuicão no Brasil: RJ (Campo Grande, Jacarepaguá/Rio de Janeiro)

Hábitat: várias gramíneas, incluindo Andropogon condensatus,

Plesiothrips sp. (citado em Monteiro, R.C.; L.A. Mound \& R.A. Zucchi, 1996. Thrips species from three counties of the State of São Paulo. Anais da Sociedade Entomológica do Brasil, 25(2): 351-353.

Distribuição no Brasil: SP (Piracicaba, São Pedro)

Hábitat: folhas de gramineas

\section{Gênero Prionothrips}

Prionothrips procerus Hood, 1954: 208 (Brasil: Nova Teutônia, SC) (ramos mortos)

Hood, J.D. 1954. Brasilian Thysanoptera V. Proceedings of the Biological Society of Washington, 67: 195-214.

Distribuição no Brasil: SC (Nova Teutônia), SP (Boracéa/Salesópolis)

Hábitat: ramos mortos

\section{Gênero Psectrothrips}

Psectrothrips interruptus (Hood, 1957: 131) (Pseudothrips) (Brasil: Nova Teutônia, SC) (planta não identificada)

Hood, J.D. 1957. New Brazilian Thysanoptera. Proceedings of the Biological Society of Washington, 70: 129-180.

Distribuição no Brasil: SC (Nova Teutônia)

Hábitat: planta não identificada

Psectrothrips longiceps (Hood, 1954: 211) (Pseudothrips) (Brasil: Serra da Cantareira, Franco da Rocha, SP) (folha de Yucca sp.)

Hood, J.D. 1954. Brasilian Thysanoptera V. Proceedings of the Biological Society of Washington, 67: 195-214.

Distribuicão no Brasil: SP (Franco da Rocha)

Hábitat: folha de Yucca sp. 


\section{Gênero Pseudodendrothrips}

Pseudodendrothrips alboniger Hood, 1952: 145 (Brasil: Nova Teutônia, SC) (Terminalia sp., plantas não identificadas)

Hood, J.D. 1952. Brasilian Thysanoptera III. Proceedings of the Biological Society of Washington, 65: 141-174.

Distribuicão no Brasil: SC (Nova Teutônia)

Hábitat: Terminalia sp.; plantas não identificadas

Pseudodendrothrips fulvus Hood, 1952: 146 (Brasil: Nova Teutônia, SC) (Dalbergia sp.)

Hood, J.D. 1952. Brasilian Thysanoptera III. Proceedings of the Biological Society of Washington, 65: 141-174.

Distribuição no Brasil: SC (Nova Teutônia)

Hábitat: Dalbergia sp.

Pseudodendrothrips stuardoi (Moulton, 1930: 73) (Graphidothrips) (Chile: San Felix) (Ficus carica)

Moulton, D. 1930. A new genus and species of Thysanoptera from Chile with notes on other species. Revista Chilena de Historia Naturale, 34: 272-275.

Distribuicão no Brasil: SP (Valinhos)

Hábitat: Ficus carica; folhagem de figueira

\section{Gênero Pseudothris}

Pseudothrips quadratus (Hood, 1954: 210) (Schistothrips) (Brasil: Nova Teutônia, SC) (várias plantas não identificadas)

Hood, J.D. 1954. Brasilian Thysanoptera V. Proceedings of the Biological Society of Washington, 67: 195-214.

Distribuicão no Brasil: SC (Nova Teutônia)

Hábitat: várias plantas não identificadas

\section{Gênero Rhamphothrips}

Rhamphothrips pandens Sakimura, 1983: 299 (Havai, Flórida e Jamaica?)

Sakimura, K. 1983. A new Rhamphothrips from Hawaii, Jamaica and Florida, and notes on $R$. pomeroyi (Thysanoptera: Thripidae). Proceedings fo the Hawaiian Entomological Society, 24: 299-303.

Distribuição no Brasil: SP (USNM)

Hábitat:

\section{Gênero Salpingothrips}

Salpingothrips minimus Hood, 1935: 158 (Panamá)

Hood, J.D. 1935. Eleven new Thripidae (Thysanoptera) from Panama. Journal of the New York Entomological Society, 43(2): 143-171.

Distribuição no Brasil:

Hábitat:

Salpingothrips sp. (espécimens do Brasil no USNM)

Distribuiç̃o no Brasil:

Hábitat:

\section{Gênero Scirtidothrips}

Scirtidothrips torquatus Hood, 1954: 207 (Brasil: Nova Teutônia, SC) (desconhecido)

Hood, J.D. 1954. Brasilian Thysanoptera V. Proceedings of the Biological Society of Washington, 67: 195-214. 
Distribuição no Brasil: SC (Nova Teutônia)

Hábitat: desconhecido

\section{Scirtidothrips sp.}

Distribuição no Brasil: SP (Piracicaba)

Hábitat: folhas de gramíneas

\section{Gênero Scirtothrips}

Scirtothrips bondari Moulton, 1933: 103, 104 (Brasil: BA) (Ipomoea batatas)

Moulton, D. 1933. The Thysanoptera of South America II. Revista de Entomologia, 3(1): 96-133.

Distribuicão no Brasil: BA

Hábitat: Ipomoea batatas

Scirtothrips manihoti (Bondar, 1924: 216) (Euthrips) (Brasil: BA) (Manihot utilissima)

Bondar, G. 1924. Dois males nas folhas da mandioca. Chacaras e Quintaes, 30: 215-219.

Distribuição no Brasil: BA, MG, RS, SP

Hábitat: folhas de mandioca (Manihot utilissima); planta desconhecida

Scirtothrips multistriatus Hood, 1954: 206 (Brasil: Belém, PA) (adventícios em ramos mortos de Hevea sp.; gramineas)

Hood, J.D. 1954. Brasilian Thysanoptera V. Proceedings of the Biological Society of Washington, 67: 195-214.

Distribuição no Brasil: PA

Hábitat: adventícios em ramos mortos de Hevea sp.; gramíneas

Scirtothrips panamensis Hood, 1935: 153 (Panamá: Barro Colorado Island, Zona do Canal) (vários brotos de arbustos não identificados crescendo em uma plantação de banana)

Hood, J.D. 1935. Eleven new Thripidae (Thysanoptera) from Panama. Journal of the New York Entomological

Society, 43(2): 143-171.

Distribuição no Brasil: SP (Piracicaba)

Hábitat: folhas de Memusa sp.

Scirtothrips sp. (citado em Costa Lima, A.M. da. 1936. In: Terceiro catálogo dos insetos que vivem nas plantas do Brasil.Ministério da Agricultura, Escola Nacional de Agronomia, Diresct. Estat. Produção, p. 111, 460p + iv)

Distribuicão no Brasil: RJ

Hábitat: folhas de Citrus

\section{Scirtothrips sp2}

Distribuição no Brasil: SP (Piracicaba)

Hábitat: folhas de mandioca

\section{Scirtothrips $\mathbf{s p}_{3}$}

Distribuição no Brasil: Jaboticabal (Sérgio de Freitas)

Hábitat: seringueira

\section{Gênero Scolothrips}

Scolothrips sexmaculatus (Pergande, 1894: 542) (Thrips) (USA: Los Angeles, Califórnia) (predando ácaro vermelho)

Pergande, T. 1894. Transactions of the St. Louis Academy, 5: 542

Distribuição no Brasil: BA, SP

Hábitat: predador de ácaro vermelho; Ipomoea sp. 


\section{Gênero Sericothrips}

Sericothrips daedalus Hood, 1954: 201 (Brasil: Nova Teutônia, SC) (Leuhea sp.)

Hood, J.D. 1954. Brasilian Thysanoptera V. Proceedings of the Biological Society of Washington, 67: 195-214.

Distribuicão no Brasil: SC (Nova Teutônia)

Hábitat: Leuhea sp.

Sericothrips fasciatus Moulton, 1938: 375 (Brasil: Viçosa, MG) (Compositae desconhecida)

Moulton, D. 1938. Thysanoptera from Minas Geraes, Brazil. Revista de Entomologia, 9(3-4): 374-382.

Distribuicão no Brasil: MG (Viçosa)

Hábitat: Compositae desconhecida

Sericothrips fimbriatus Hood, 1954: 200 (Brasil: Nova Teutônia, SC) (folha de Vitis sp.)

Hood, J.D. 1954. Brasilian Thysanoptera V. Proceedings of the Biological Society of Washington, 67: 195-214.

Distribuição no Brasil: SC (Nova Teutônia)

Hábitat: folhas de Vitis sp.

Sericothrips flavens Moulton, 1941: 316 (Brasil: MG) (arbusto desconhecido entre as árvores)

Moulton, D. 1941. Thysanoptera from Minas Geraes, Brazil (second paper). Revista de Entomologia, 12(1-2): 314322.

Distribuição no Brasil: MG

Hábitat: arbusto desconhecido entre as árvores

Sericothrips hemileucus Hood, 1952: 144 (Brasil: Boracéa, Salesópolis, SP) (ramos mortos)

Hood, J.D. 1952. Brasilian Thysanoptera III. Proceedings of the Biological Society of Washington, 65: 141-174.

Distribuicão no Brasil: SP (Boracéa/Salesópolis), SC (Nova Teutônia)

Hábitat: ramos mortos; Solanum sp.

Sericothrips maculicollis Hood, 1954: 203 (Brasil: Serra da Cantareira, Franco da Rocha, SP) (diversas vegetações)

Hood, J.D. 1954. Brasilian Thysanoptera V. Proceedings of the Biological Society of Washington, 67: 195-214.

Distribuicão no Brasil: SP (Franco da Rocha)

Hábitat: diversas plantas

Sericothrips paraensis Hood, 1954: 205 (Brasil: Belém, PA) (desconhecido: coletando com rede)

Hood, J.D. 1954. Brasilian Thysanoptera V. Proceedings of the Biological Society of Washington, 67: 195-214.

Distribuicão no Brasil: PA (Belém)

Hábitat: desconhecido

Sericothrips ruginosus Hood, 1954: 202 (Brasil: Itaguai, RJ) (uma trepadeira)

Hood, J.D. 1954. Brasilian Thysanoptera V. Proceedings of the Biological Society of Washington, 67: 195-214.

Distribuicão no Brasil: RJ (Itaguai)

Hábitat: uma trepadeira"

Sericothrips sidae Crawford, 1944: 200 (Brasil: São Paulo, SP) (Sida rhombifolia)

Crawford, J.C. 1944. A new Sericothrips from Brazil (Thysanoptera: Thripidae). Proceedings of the Entomological Society of Washington, 46(7): 200-201.

Distribuição no Brasil: SP (São Paulo)

Hábitat: Sida rhombifolia 
Sericothrips varius Moulton, 1941: 317 (Brasil: MG) (flores de rosa)

Moulton, D. 1941. Thysanoptera from Minas Geraes, Brazil (second paper). Revista de Entomologia, 12(1-2): 314322.

Distribuição no Brasil: MG

Hábitat: flores de roseira

\section{Gênero Stenchaetothrips}

Stenchaetothrips biformis (Bagnall, 1913: 237) (Bagnallia) (Inglaterra: Weston-on-the-Green, Oxford)

Bagnall, R.S. 1913. Further notes on new and rare British Thysanoptera (Terebrantia) with descriptions of new species. Journal of Economic Biology, 8(4): 231-240.

Sinônimo: Thrips (Bagnallia) oryzae (Williams; 1916: 353) (South Índia: Madurantakan) = Chloëthrips oryzae (Williams)

Williams, C.B. 1916. Thrips oryzae, sp. nov., injurious to rice in India. Bulletin of Entomological Research, 6(4): 353-355.

Distribuicão no Brasil: RS

Hábitat: espigas de arroz

Stenchaetothrips brasiliensis (Hood, 1954: 212) (Anaphidothrips) (Brasil: Campo Grande, RJ)

Hood, J.D. 1954. Brasilian Thysanoptera V. Proceedings of the Biological Society of Washington, 67: 195-214.

Distribuição no Brasil: RJ (Campo Grande)

Hábitat: Andropogon sp. (provavelmente A. condensatus)

Stenchaetothrips minutus (Deventer, 1906: 276, 281) (Thrips) (Java)

Deventer, W. van. 1906. Handboek ten dienste van de Suikerriet-Cultur en de Rietsuiker-Fabricabe op Java, 2: 275-

282.

Sinônimos:

Thrips minutus var. puttemansi Costa Lima, 1926: 32 (Brasil: SP) (bainha das folhas de cana-de-açúcar)

Costa Lima, A.M. da. 1926. A proposito de uma communicação do Dr. Puttemans sôbre o mosaico da canna de assucar. Chácaras e Quintaes, 34(1): 30-32.

Thrips saccharoni Moulton, 1928: 111 (Havai: Honolulu) (cana-de-açúcar)

Moulton, D. 1928. Thysanoptera of the Hawaiian Islands. Proceedings of the Hawaiian Entomological Society, 7: 105-134.

Distribuicão no Brasil: RJ, RS, SP (Rafard, Piracicaba, Jaboticabal)

Hábitat: folhas novas de cana-de-açúcar e de cana-taquara; folhas de pimentão (Capsicum annuum); folhas de gramineas; Sorghum bicolor, folhas de gramineas

\section{Gênero Taeniothrips}

Taeniothrips inconsequens (Uzel, 1895: 117) ((Physopus) (Bohemia) (?Prunus sp.)

Uzel, H. 1895. Monographie der Ordnung Thysanoptera. 472p. Königrätz.

Sinônimos:

Taeniothrips sp. Gb

Taeniothrips xanthius (Williams, 1917: 59) (Trinidade: Port of Spain) (orquideas Cattleya spp.)

Williams, C.B. 1917. A new thripsdamaging orchids in the West Ubdues. Bulletin of Entomological Research, 8(1):

59-61.

Distribuicão no Brasil: RS, SP

Hábitat: flores de pereira 


\section{Gênero Thrips}

Thrips australis (Bagnall, 1915: 592) (Isoneurothrips) (Westem Austrália: Mundaring Weir, Darling Range, Perth) (flores de Acacia sp.)

Bagnall, R.S. 1915. Brief descriptions of new Thysanoptera VI. Annals and Magazine of Natural History, $8^{\text {th }}, 15$ : 588-597.

Distribuicão no Brasil: MG, SP (Piracicaba)

Hábitat: folhas e flores de Eucalyptus spp. em ramos quebrados

Thrips palmi Kany, 1925: 10 (Sumatra: Medan) (fumo Nicotiana tabacum)

Karny, H.H. 1925. Die an tabak auf Java und Sumatra angetroffenen Blasenfüsser (Thysanoptera). Bulletin van het Deli Proefstataion, Medan, Sumatra, 23: 1-55.

Distribuicão no Brasil: BA (Teixeira de Freitas), DF (Brasília e arredores), GO (Goiânia, Luziânia, Santa Helena), MG (Bueno Brandão), PR (Assaí, Morretes), RJ (Cachoeiras de Macacu e Vassouras), RR (Normandia, Apiaú), SP (Artur Nogueira, Atibaia, Conchal, Elias Fausto, Holambra, Itapetininga, Itapira, Jaguariúna, Marília, Monte Mor, Paranapanema, Paulinia, Piedade, Pinhalzinho, Registro, São Miguel Arcanjo, Sete Barras)

Hábitat: folhas e flores de tomate (Lycopersicon esculentum); folhas, flores e frutos de pimentão (Capsicum annuum); folhas e flores de crisântemo (Chrysanthemum sp.); folhas de batata (Solanum tuberosum); folhas e frutos de berinjela (Solanum melongena); feijão (Phaseolus vulgaris); maria-pretinha (Solanum nigrum); folhas de pepino; folhas de feijão de corda (Vigna unguiculata); folhas de melancia; jiló; vagem; banana

Thrips simplex (Morison, 1930: 12) (Physothrips) (Austrália: Urrbrae) (flores de cravo) Morison, G.D. 1930. On a collection of Thysanoptera from South Australia. Bulletin of Entomological Research, 21(1): 9-14.

Distribuicão no Brasil: RJ, RS, SP (Capão Bonito)

Hábitat: folhas e flores de gladíolo

Thrips tabaci Lindeman, 1889: 61 (Basarabia)

Lindeman, K. 1889. Die schälichsten insekten des tabak in Bessarabien. Bulletin de la Société Imperiale des Naturalistes de Moscou, 1(15): 61-75.

Distribuição no Brasil: BA (Jequié), MG, ), PR (Londrina, Bandeirantes), RJ, RS, SC (Ituporanga), SP (Campinas, Paulinia, Piracicaba)

Hábitat: folhas de aspargo (Asparagus officinalis); folhas de cebolinha (Allium fistulosum); folhas de alho (Allium sativum); folhas de cebola (Allium cepa); folhas e flores de soja (Glycine max); gérbera 


\title{
Lista dos Tubulifera (Thysanoptera) constatados no Brasil
}

\author{
Phlaeothripidae - Idolothripinae \\ Gênero Accallurothrips
}

Accallurothrips conifer (Hood, 1925: 67) (Pygothrips) (Trinidade) (ramos mortos de Lagerstroemia sp. infestadas com bromeliáceas)

Hood, J.D. 1925. New Tropical Thysanoptera collected By C.B. Williams. Psyche, 32(1): 48-69.

Distribuicão no Brasil: AM (Lago Janauaca)

Habitat: Pyrethrum fogging

Accallurothrips fasciolatus (Hood, 1952: 165) (Pygothrips) (Brasil: Jacarepaguá, Rio de Janeiro, RJ) (ramos mortos)

Hood, J. D. 1952. Brasilian Thysanoptera III. Proceedings of the Biological Society of Washington 65: 141-174.

Distribuição no Brasil: RJ (JacarepaguáRio de Janeiro, Petrópolis)

Habitat: ramos mortos

Accallurothrips quadraticeps (Hood, 1952: 166) (Pygothrips) (Brasil: Campo Grande, RJ) (ramo morto) Hood, J. D. 1952. Brasilian Thysanoptera III. Proceedings of the Biological Society of Washington 65: 141-174. Distribuicão no Brasil: RJ (Campo Grande)

Habitat: ramo morto

\section{Gênero Actinothrips}

Actinothrips bondari Hood, 1928: 147 (Brasil: BA) (folhas mortas de cacau)

Hood, J.D. 1928. A new Actinothrips (Thysanoptera) from Brazil. Bulletin of the Brooklyn Entomological Society, 23(3): 147-150.

Distribuicão no Brasil: BA

Habitat: folhas mortas de cacau

Actinothrips femoralis Hood, 1950: 5 (Brasil: São Carlos, SP) (ramos mortos com folhas)

Hood, J.D. 1950. Brasilian Thysanoptera II. Revista de Entomologia, 21(1-2): 1-113.

Distribuição no Brasil: SP (São Carlos)

Habitat: ramos mortos com folhas, folha morta de palmácea no solo

Actinothrips gargantua De Santis, 1960: 57 (Brasil: Barueri, SP) (desconhecido)

De Santis, L. 1960. Un nuevo Tisanóptera gigante del Brasil. Actas y trabajos del Primer Congreso Sudamericano de zoologia, 3: 57-60.

Distribuição no Brasil: SP (Barueri)

Habitat: desconhecido

Actinothrips pedalis Hood, 1949: 76 (Brasil: Itanhaém, SP) (folhas mortas nos ramos)

Hood, J.D. 1949. Brasilian Thysanoptera I. Revista de Entomologia, 20(1-3): 3-88

Distribuição no Brasil: SP (Itanhaém), RJ (Ilha da Gipóia, próximo à Angra dos Reis, RJ)

Habitat: folhas mortas em ramos

Actinothrips $\mathrm{sp}_{1}$. trichaetus grupo

Distribuição no Brasil: SP (Piracicaba)

Habitat: saco-de-Adão 


\section{Actinothrips sp.}

Distribuicão no Brasil: SC (Nova Teutônia)

Habitat: desconhecido

\section{Gênero Allothrips}

Allothrips brasilianus Hood, 1956(1955): 101 (Brasil: Jacarepaguá, Rio de Janeiro, RJ) (ramos mortos)

Hood, J.D. 1956(1955). Brasilian Thysanoptera VI. Revista Brasileira de Entomologia, 4: 51-160.

Distribuição no Brasil: RJ (Jacarepaguá/Rio de Janeiro)

Habitat: ramos mortos

\section{Gênero Anactinothrips}

Anactinothrips fuscus Moulton, 1933: 418 (Brasil: ES) (planta desconhecida)

Moulton, D. 1933. The Thysanoptera of South America IV. Revista de Entomologia, 3(3): 385-419.

Distribuicão no Brasil: ES

Habitat: planta desconhecida

Anactinothrips gibbifer zur Strassen, 1980: 48 (Brasil: Manaus,AM) (Eschweilera sp.)

zur Strassen, R. 1980. Anactinothrips gibbifer n. sp. aus Baumlronen im Amazonas-Gebiet (Insecta: Thysanoptera:

Phlaeothripidae). Senckenbergiana Biologica, 61: 47-56.

Distribuicão no Brasil: AM (Manaus)

Habitat: Eschweilera sp.

Anactinothrips gustaviae Mound \& Palmer, 1983: 143 (Panamá)

Mound, L.A. \& J.M. Palmer. 1983. The generic and tribal classification of spore-feeding Thysanoptera (Phlaeothripidae: Idolothripinae). Bulletin of the British Museum (Natural History), Entomology, 46: 1-174.

Distribuicão no Brasil: AM (Manaus)

Habitat:

Anactinothrips handlirschii (Schmutz, 1909: 275) (Ophidothrips) (Brasil: RS) (desconhecido)

Schmutz, K. 1909. Zur Kenntnis einiger neuen Thysanopterengenera (Tubulifera). Annalen des Naturhistorischen Museums, Wien, 23(3-4): 273-281.

Distribuicão no Brasil: RS

Habitat: desconhecido

Anactinothrips nigricornis Hood, 1936: 143 (Guiana Britânica: Rockstone) (desconhecido)

Hood, J.D. 1936. Two new Anactinothrips from South America (Thysanoptea). Proceedings of the Royal Entomological Society of London, Series B (Taxonomy), 5(7): 143-147.

Sinônimo: Anactinothrips borgmeieri Hood, 1950: 34 (Brasil: Lar das Cegas, Jacarepaguá, Rio de Janeiro, RJ) (ramos mortos)

Hood, J.D. 1950. Brasilian Thysanoptera II. Revista de Entomologia, 21(1-2): 1-113.

Distribuicãa no Brasil: RJ (Jacarepaguá/Rio de Janeiro, Petrópolis)

Habitat: ramos mortos

Anactinothrips silvicola Hood, 1952: 167 (Brasil: Belém, PA) (folhas mortas de Astrocaryum sp., Heliconia sp. e outras, na floresta)

Hood, J.D. 1952. Brasilian Thysanoptera III. Proceedings of the Biological Society of Washington, 65: 141-174.

Distribuição no Brasil: PA (Belém)

Habitat: folhas mortas de Astrocaryrum sp., Heliconia sp. e outras, na floresta. 


\section{Gênero Compsothrips}

Compsothrips bicolor Priesner, 1921: 213 (Paraguai) (desconhecido)

Priesner, H. 1921. Neue und wenig bekannte Thysanopteren der neotropischen Fauna aus der Sammlung des Berliner Zoologischen Museums. Deutsche Entomologische Zeitung, 3: 187-223.

Distribuicão no Brasil: PE (Recife)

Habitat:

Compsothrips brasiliensis (Hood, 1952: 166) (Oedaleothrips) (Brasil: Serra da Cantareira, Franco da Rocha, $\mathrm{SP}$ ) (gramínea)

Hood, J.D. 1952. Brasilian Thysanoptera III. Proceedings of the Biological Society of Washington, 65: 141-174.

Distribuicão no Brasil: SP (Franco da Rocha)

Habitat: graminea

Compsothrips graminis (Hood, 1936: 265) (Oedaleothrips) (British West Índias, Patos Island, próximo a Trinidade) (graminea)

Hood, J.D. 1936. Studies in Neotropical Thysanoptera I. Revista de Entomologia 6(2): 248-279.

Distribuicão no Brasil:

Habitat: gramínea

\section{Gênero Cyphothrips}

Cyphothrips dorsalis Hood, 1952: 172 (Brasil: Belém, PA) (folhas mortas de assai Euterpe oleracea)

Hood, J.D. 1952. Brasilian Thysanoptera II. Proceedings of the Biological Society of Washington, 65: 141-174.

Distribuicão no Brasil: PA (Belém)

Habitat: folhas mortas de assai (Euterpe oleracea)

\section{Gênero Diceratothrips}

Diceratothrips bicornis Bagnall, 1908: 194 (Brasil: Santarem, PA) (desconhecido)

Bagnall, R.S. 1908. On some new genera and species of Thysanoptera. Transactions of the Natural History Society of Northumberland, 3(1): 183-217.

Distribuição no Brasil: AM (Ilha Marchantia)

Habitat: desconhecido, sob casca de árvore

Diceratothrisps cornutus Hood, 1952: 156 (Brasil: Belém, PA) (graminea alta)

Hood, J.D. 1952. Brasilian Thysanoptera III. Proceedings of the Biological Society of Washington, 65: 141-174.

Distribuição no Brasil: PA (Belém)

Habitat: gramínea e ramo morto de caju

Diceratothrisps nigricauda (Hood, 1925: 67) (Pygothrips) (Trinidade) (ramos)

Hood, J.D. 1925. New neotropical Thysanoptera collected by C.B. Williams. Psyche, 32(1): 48-69.

Distribuicão no Brasil: AM (Lago Janauaca)

Habitat: ramos, "Pyrethrum fogging"

Diceratothrisps robustus (Schmutz, 1909: 278) (Eulophothrips) (Brasil: RS) (desconhecido)

Schmutz, K. 1909. Zur Kenntnis einiger neuen Thysanopterengenera (Tubulifera). Annalen des Naturhistorischen Museums, Wien, 23(3-4): 273-281. 342-347.

Distribuicão no Brasil: RS

Habitat: desconhecido 


\section{Gênero Diplacothrips}

Diplacothrips piceus Hood, 1952: 161 (Brasil: Belém, PA) (ramos mortos)

Hood, J.D. 1952. Brasilian Thysanoptera III. Proceedings of the Biological Society of Washington, 65: 141-174.

Distribuição no Brasil: PA (Belém)

Habitat: ramos mortos

\section{Gênero Elaphrothrips}

Elaphrothrips amazonicus Johansen, 1979: 95 (Peru: Shapajilla, a $11 \mathrm{~km}$ a NE de Tingo Maria) (ramos e folhas secas)

Johansen, RM. 1978. Seis nuevas especies de Elaphrothrips Buffa, 1909 (Thysanoptera: Phlaeothripidae) de Brasil y Perú. Anales del Instituto de Biología, Universidad Nacional de México, Serie Zoologia, 49(1): 95-114.

Distribuição no Brasil:

Habitat:

Elaphrothrips angustifrons (Bergroth, 1888: xxx) (Phloeothrips) (Brasil: Blumenau, SC) (? Drosera sp.)

Bergroth, E. 1888. Diagnose d'une nouvelle spèce de Thysanoptères. Annales de lá Societé Entomologiqué de Belgique, Bulletin ou Comptes-Rendus des Séances de la Societé Entomologiqué de Belgique, 3(98): xxx-xxai.

Distribuicão no Brasil: SC (Blumenau)

Habitat: desconhecido (? Drosera sp.)

Elaphrothrips borgmeieri Hood, 1956(1955): 62 (Brasil: Belém, PA) (trepadeiras e gramíneas altas mortas misturadas)

Hood, J.D. 1956(1955). Brasilian Thysanoptera VI. Revista Brasileira de Entomologia, 4: 51-160.

Distribuicão no Brasil: PA (Belém)

Habitat: gramíneas mortas, folhas mortas e ramos mortos de trepadeiras e gramineas altas

Elaphrothrips brasiliensis Johansen, 1978: 104 (Brasil) (não mencionado)

Johansen, R.M. 1978. Seis nuevas especies de Elaphrothrips Buffa, 1909 (Thysanoptera: Phlaeothripidae) de Brasil y Perú. Anales del Instituto de Biología, Universidad Nacional de México, Serie Zoologia, 49(1): 95-114.

Distribuição no Brasil: não mencionado

Habitat: não mencionado

Elaphrothrips costalimai Hood, 1956(1955): 57 (Brasil: Jacarepaguá, Rio de Janeiro, RJ) (folhas mortas e dependuradas de Dahlia sp.)

Hood, J.D. 1956(1955) Brasilian Thysanoptera VI. Revista Brasileira de Entomologia, 4: 51-160.

Distribuição no Brasil: RJ (Jacarepaguá/Rio de Janeiro), PA (Praia de Icoaraci/Belém), SP (Barueri)

Habitat: folhas mortas e dependuradas de Dahlia sp., folhas secas de Sechium edule

Elaphrothrips gracilis Moulton, 1933: 409, 410 (Brasil: Afonso Cláudio, ES) (desconhecido: coletando com rede)

Moulton, D. 1933. The Thysanoptera of South America IV. Revista de Entomologia, 3(3): 385-419.

Distribuicãa no Brasil: ES (Afonso Cláudio)

Habitat: desconhecido

Elaphrothrips laevicollis (Bagnall, 1910: 375) (Dicaiothrips) (Venezuela: Los Tejes) (sem dado)

Bagnall, R.S. 1910. A contribution towards a knowledge of the neotropical Thysanoptera. Journal of the Linnean

Society, Zoology, 30(201): 369-387.

Distribuicão no Brasil: SP (Piracicaba)

Habitat: vagens abertas de Sesbania sp.

Elaphrothrips macateei Hood, 1956(1955): 60 (Brasil: Belém, PA) (ramo morto de uma árvore não identificada)

Hood, J.D. 1956(1955). Brasilian Thysanoptera VI. Revista Brasileira de Entomologia, 4: 51-160. 
Distribuicão no Brasil: PA (Belém)

Habitat: ramo morto de uma árvore não identificada

Elaphrothrips nitidus (Bagnall, 1910: 372) (Dicaiothrips) (Brasil: Serra dos Órgãos, RJ) (desconhecido) Bagnall, R.S. 1910. A contribution towards a knowledge of the neotropical Thysanoptera. Journal of the Linnean Society, Zoology, 30(201): 369-387.

Distribuicão no Brasil: RJ (Serra dos Órgãos, altitude 600-1000 m)

Habitat: desconhecido

Elaphrothrips palustris (Hood, 1952: 168) (Palinothrips) (Brasil: São Carlos, SP) (gramínea em brejo) Hood, J.D. 1952. Brasilian Thysanoptera III. Proceedings of the Biological Society of Washington, 65: 141-174. Distribuicão no Brasil: SP (São Carlos)

Habitat: gramínea em brejo

Elaphrothrips schottii (Heeger, 1852: 139) (Thrips) (Brasil) (desconhecido)

Heeger, E. 1852. Beiträge zur Naturgeschichte der Physapoden (Blassenfusse). Sitzungsberichte der Kaiserlichen Akademie der Wissenschaften, Wien, 9: 123-141.

Distribuição no Brasil: sem dado

Habitat: desconhecido

Elaphrothrips snodgrassi Hood, 1956(1955): 66 (Brasil: Serra da Cantareira, Franco da Rocha, SP) (ramos mortos caídos com folhas)

Hood, J.D. 1956(1955). Brasilian Thysanoptera VI. Revista Brasileira de Entomologia, 4: 51-160.

Distribuicão no Brasil: SP (Franco da Rocha)

Habitat: ramos caídos com folhas

Elaphrothrips unicolor Moulton, 1933: 415 (Brasil: ?BA - coletado por Bondar) (dado não mencionado)

Moulton, D. 1933. The Thysanoptera of South America IV. Revista de Entomologica, 3(3): 385-419.

Distribuicão no Brasil: BA?, SP (Boracéa/Salesópolis), RJ (Ilha da Gipoia, próximo à Angra dos Reis), SC (Nova Teutônia), PR (Curitiba)

Habitat: ramos e folhas mortas; galhos ocos e casca de Schinus terebinthifolius

Elaphrothrips ?propinquus (Bagnall, 1910: 377) (Dicaiothrips) (Venezuela: Los Trincheras) (não mencionado)

Bagnall, R.S. 1910. A contribution towards a knowledge of the neotropical Thysanoptera. Journal of the Linnean Society, Zoology, 30(201): 369-387.

Distribuição no Brasil: AM (Manaus)

Habitat: folhas jovens de guaraná (Paullinia cupana)

Elaphrothrips sp. (citado em Monteiro et al. 1996)

Distribuição no Brasil: SP (Piracicaba, Ubatuba)

Habitat: folhas de pupunha (Bactris gasipaes), vagens de Sesbania sp.

\section{Gênero Ethirothrips}

Ethirothrips firmus (Hood, 1952: 162) (Gastrothrips) (Brasil: Itanhaém, SP) (ramos mortos)

Hood, J.D. 1952. Brasilian Thysanoptera II. Proceedings fo the Biological Society of Washington, 65: 141-174.

Distribuicão no Brasil: SP (Itanhaém)

Habitat: ramos mortos 


\section{Gênero Gastrothrips}

Gastrothrips abditus Hood, 1935: 177 (Panamá: Barro colorado Island, Zona do Canal) (em cavidades de ramos mortos de uma árvores não determinada)

Hood, J.D. 1935. Some new or little known Thysanoptera of the family Phlaeothripidae. Revista de Entomologia, 5(2): 159-199.

Sinônimos:

Hoplothrips brasiliensis Moulton, 1938: 378 (Brasil: Viçosa, MG) (galhos mortos de mandioca)

Moulton, D. 1938. Thysanoptera from Minas Geraes, Brazil. Revista de Entomologia, 9(3-4): 374-382.

Probolothrips hambletoni Moulton, 1941: 320 (Brasil: MG) (hastes mortas de mandioca)

Moulton, D. 1941. Thysanoptera from Minas Geraes, Brazil (second paper). Revista de Entomologia, 12(1-2): 314322.

Distribuicão no Brasil: RJ (Jacarepaguá/Rio de Janeiro, Itaguai, Campo Grande, Tha da Gipóia), SP (Serra da Cantareira), MG (Viçosa)

Habitat: hastes e ramos mortos de mandioca, burrous e outros recesses em ramos mortos

Gastrothrips fumipennis Hood, 1952: 163 (Brasil: Nova Teutônia, SC) (ramos secos)

Hood, J.D. 1952. Brasilian Thysanoptera III. Proceedings fo the Biological Society of Washington, 65: 141-174.

Distribuicãa no Brasil: SC (Nova Teutônia)

Habitat: ramos secos

Gastrothrips mandiocae (Moulton, 1941: 321) (Dichaetothrips) (Brasil: MG) (ramos mortos de mandioca) Moulton, D. 1941. Thysanoptera from Minas Geraes, Brazil (second paper). Revista de Entomologia, 12(1-2): 314322.

Sinônimo: Gastrothrips oeceticola De Santis, 1943 ou 1940?: 92 (Argentina, Coronel Vidal, Província de Buenos Aires)

De Santis, L. 1940. Notas tisanopterologicas referidas a especies nuevas y conocidas de la Republica Argentina. Revista de la Facultad de Agronomia (Tercera Época), Universidad Nacional de La Plata, 25: 89-96.

Distribuicão no Brasil: MG, SC (Nova Teutônia), SP (São Carlos, Itanhaém), RJ (Tha da Gipóia)

Habitat: ramos mortos de mandioca, ramos mortos

Gastrothrips procerus Hood, 1956(1955): 99 (Brasil: Nova Teutônia, SC) (ramos secos)

Hood, J.D. 1956(1955). Brasilian Thysanoptera VI. Revista Brasileira de Entomologia, 4: 51-160.

Distribuição no Brasil: SC (Nova Teutônia), PR (Ponta Grossa)

Habitat: ramos secos; tufos de gramineas

Gastrothrips sp.

Distribuicão no Brasil: SP (Piracicaba)

Habitat: vagens abertas de Sesbania sp.

\section{Gênero Neosmerinthothrips}

Neosmerinthothrips annulipes (Hood, 1950: 13) (Gastrothrips) (Brasil: Jacarepaguá, Rio de Janeiro, RJ) (ramos mortos)

Hood, J.D. 1950. Brasilian Thysanoptera II. Revista de Entomologia, 21(1-2): 1-113.

Distribuição no Brasil: RJ (Jacarepaguá/Rio de Janeiro, Itaguai)

Habitat: ramos mortos

Neosmerinthothrips diversicolor (Moulton, 1933: 404) (Galactothrips) (Brasil: BA) (Galactia sp.)

Moulton, D. 1933. The Thysanoptera of South America IV. Revista de Entomologia, 3(3): 385-419.

Distribuição no Brasil: BA

Habitat: Galactia sp. 
Neosmerinthothrips hamiltoni Mound \& Palmer, 1983: 46 (Brasil: Campus da FFCLRP, Ribeirão Preto, SP) (galho oco de Glyricidia sp.)

Mound, L.A. \& J.M. Palmer. 1983. The generic and tribal classification of spore-feeding Thysanoptera (Phlaeothripidae: Idolothripinae). Bulletin of the British Museum (Natural History), Entomology Series, 46(1): 1174.

Distribuição no Brasil: AM?, SP (Ribeirão Preto)

Habitat: galho oco de Glyricidia sp.

Neosmerinthothrips paulistarum (Hood, 1950: 25) (Gastrothrips) (Brasil: São Carlos, SP) (ramo morto)

Hood, J.D. 1950. Brasilian Thysanoptera II. Revista de Entomologia, 21(1-2): 1-113.

Distribuicão no Brasil: SP (São Carlos)

Habitat: ramo morto

Neosmerinthothrips picticornis (Hood, 1936: 272) (Gastrothrips) (Brasil: Angra dos Reis, RJ) (ramos mortos de fruta-do-donde Anona squamosa)

Hood, J.D. 1936. Studies in Neotropical Thysanoptera I. Revista de Entomologia, 6(2): 248-279

Distribuição no Brasil: RJ (Angra dos Reis, Petrópolis), SP (Itanhaém, Serra da Cantareira/Franco da Rocha)

Habitat: ramos mortos de Anona squamosa, ramos mortos de várias espécies

Ref.: Hood, J.D. 1950. Brasilian Thysanoptera II. Revista de Entomologia, 21(1-2): 1-113

Neosmerinthothrips plaumanni (Hood, 1950: 20) (Gastrothrips) (Brasil: Nova Teutônia, SC) (ramos secos, ramos mortos)

Hood, J.D. 1950. Brasilian Thysanoptera II. Revista de Entomologia, 21(1-2): 1-113.

Distribuição no Brasil: SC (Nova Teutônia)

Habitat: ramos secos, ramos mortos

Neosmerinthothrips variipes (Hood, 1950: 16) (Gastrothrips) (Brasil: Jacarepaguá, Rio de Janeiro, RJ) (reentrâncias em ramos mortos)

Hood, J.D. 1950. Brasilian Thysanoptera II. Revista de Entomologia, 21(1-2): 1-113.

Distribuição no Brasil: RJ (Jacarepaguá/Rio de Janeiro), SP (São Carlos)

Habitat: ramos mortos

Neosmerinthothrips? sp. (citado em Monteiro et al., 1996)

Distribuição no Brasil: SP

Habitat:

\section{Gênero Phacothrips}

Phacothrips ocelloides (Hood, 1950: 9) (Gastrothrips) (Brasil: Jacarepaguá, Rio de Janeiro, RJ) (ramos mortos, onde a espécie aparentemente frequente em escavações de Coleoptera)

Hood, J.D. 1950. Brasilian Thysanoptera II. Revista de Entomologia, 21(1-2): 1-113.

Distribuiccão no Brasil: RJ (Jacarepaguá/Rio de Janeiro, Campo Grande), SP (Itanhaém, São Carlos), MG (Viçosa)

Habitat: ramos mortos

Phacothrips? sp. (citado em Monteiro et al., 1996)

Distribuicão no Brasil: SP (Piracicaba)

Habitat: ramo morto no chao, com liquen

\section{Gênero Pygothrips}

Pygothrips callipygus Hood, 1952: 164 (Brasil: Belém, PA) (ramos mortos de Hevea sp.)

Hood, J.D. 1952. Brasilian Thysanoptera III. Proceedings fo the Biological Society of Washington, 65: 141-174.

Distribuicão no Brasil: PA (Belém)

Habitat: ramo morto de Hevea sp. 
Pygothrips longiceps Hood, 1952: 164 (Brasil: Campo Grande, RJ) (várias gramíneas, em urn pomar de laranja, em uma colina)

Hood, J.D. 1952. Brasilian Thysanoptera III. Proceedings fo the Biological Society of Washington, 65: 141-174.

Distribuição no Brasil: RJ (Campo Grande), MG (Viçosa)

Habitat: várias gramíneas

Pygothrips magnicauda Hood, 1954: 45 (Brasil: Belém, PA) (ramos mortos de Hevea sp.)

Hood, J.D. 1954. Brasilian Thysanoptera IV. Proceedings of the Biological Society of Washington, 67: 17-54.

Distribuicão no Brasil: PA (Belém), ?AM (Manaus)

Habitat: ramos mortos de Hevea sp.

\section{Gênero Saurothrips}

Saurothrips assai Hood, 1952: 171 (Brasil: Belém, PA) (folhas mortas de assaí Euterpe oleracea)

Hood, J.D. 1952. Brasilian Thysanoptera III. Proceedings fo the Biological Society of Washington, 65: 141-174.

Distribuição no Brasil: PA (Belém)

Habitat: folhas mortas de assai Euterpe oleracea

\section{Gênero Zactinothrips}

Zactinothrips elegans Hood, 1936: 447 (Peru: arredores de Sani Beni - floresta tropical, região de Satipo, Dept. Yunin, 840m) (ramos secos, com folhas secas)

Hood, J.D. 1936. Studies in Neotropical Thysanoptera II. Revista de Entomologia, 6(3-4): 424-460.

Distribuição no Brasil: $0^{\circ} 35^{\prime} \mathrm{N}, 66^{\circ} 41^{\prime} \mathrm{W}$

Habitat: ramos secos, com folhas secas.

Zactinothrips modestus Hood, 1941: 230 (Peru: Rioja (Minas, Rio Seco), Dept. San Martin, floresta a cerca de $900 \mathrm{~m}$ de altura) (ramos e folhas mortas)

Hood, J.D. 1941. A century of new American Thysanoptera II. Revista de Entomologia, 12(1-2): 139-243.

Distribuiç̃o no Brasil:

Habitat:

\section{Gênero Zeugmatothrips}

Zeugmatothrips borgmeieri Hood, 1949: 80 (Brasil: Jacarepaguá, Rio de Janeiro, RJ) (folhas mortas de canade-açúcar - confirmar)

Hood, J.D. 1949. Brasilian Thysanoptera I. Revista de Entomologia, 20(1-3): 3-88.

Distribuição no Brasil: RJ (JacarepaguáRio de Janeiro), SP (Itanhaém)

Habitat: folhas mortas de cana-de-açúcar, graminea morta, folhas mortas e outros restos no solo

Zeugmatothrips cinctus Hood, 1952: 170 (Brasil: Belém, PA) (folhas mortas de vários gêneros de palmáceas e Heliconia psittacorum)

Hood, J.D. 1952. Brasilian Thysanoptera III. Proceedings fo the Biological Society of Washington, 65: 141-174.

Distribuição no Brasil: PA (Belém)

Habitat: folhas mortas de vários gêneros de palmáceas e Heliconia psittacorum, folhas mortas de Astrocaryum murumure

Zeugmatothrips femoralis Hood, 1952: 169 (Brasil: Belém, PA) (ramo morto de Hevea brasiliensis caido no chão, na sombra)

Hood, J.D. 1952. Brasilian Thysanoptera III. Proceedings fo the Biological Society of Washington, 65: 141-174.

Distribuicão no Brasil: PA (Belém)

Habitat: ramo morto de Hevea brasiliensis 
Zeugmatothrips gracilis Hood, 1952: 171 (Brasil: Belém, PA) (graminea cortada)

Hood, J.D. 1952. Brasilian Thysanoptera III. Proceedings fo the Biological Society of Washington, 65: 141-174.

Distribuicão no Brasil: PA (Belém)

Habitat: graminea cortada

Zeugmatothrips mumbaca Hood, 1952: 169 (Brasil: Belém, PA) (folhas mortas de Astrocaryum mumbaca, em uma densa capoeira upland)

Hood, J.D. 1952. Brasilian Thysanoptera III. Proceedings fo the Biological Society of Washington, 65: 141-174.

Distribuição no Brasil: PA (Belém)

Habitat: folhas mortas de Astrocaryum mumbaca

Zeugmatothrips niger Hood, 1952: 168 (Brasil: Belém, PA) (folhas mortas de assai Euterpe oleracea)

Hood, J.D. 1952. Brasilian Thysanoptera III. Proceedings fo the Biological Society of Washington, 65: 141-174.

Distribuição no Brasil: PA (Belém)

Habitat: folhas mortas de assai (Euterpe oleracea)

Zeugmatothrips pallidulus Hood, 1958: 225 (Brasil: Linha Falcão, SC) (folhas caídas)

Hood, J.D. 1958. A new Zeugmatothrips from Brazil (Thysanoptera, Phlaeothripidae). Proceedings of the

Entomological Society of Washington, 60(5): 225-228.

Distribuicão no Brasil: SC (Linha Falcão)

Habitat: folhas caídas

Zeugmatothrips peltatus Hood, 1949: 85 (Brasil: Boracéa, 850m, município de Salesópolis, SP) (gramineas mortas no chão)

Hood, J.D. 1949. Brasilian Thysanoptera I. Revista de Entomologia, 20(1-3): 3-88.

Distribuição no Brasil: SP (Boracéa/Salesópolis)

Habitat: gramineas mortas no chão

\section{Phlaeothripidae - Phlaeothripinae}

\section{Gênero Acantothrips}

Acantothrips amoenus Hood, 1949: 45 (Brasil: Jacarepaguá, Rio de Janeiro, RJ) (ramos mortos)

Hood, J.D. 1949. Brasilian Thysanoptera I. Revista de Entomologia, 20(1-3): 3-88.

Distribuição no Brasil: RJ (Jacarepaguá/Rio de Janeiro, Ilha da Gipoia)

Habitat: ramos mortos

Acantothrips palmi Hood, 1958: 117 (Brasil: Nova Teutônia, SC) (ramos mortos)

Hood, J.D. 1958. Acantothrips palmi, a new thrips from Brazil (Thysanoptera: Phlaeothripidae). Entomological News, 69(5): 117-121.

Distribuicão no Brasil: SC (Nova Teutônia)

Habitat: ramos mortos

\section{Gênero Adraneothrips}

Adraneothrips abdominalis Hood, 1925: 55 (West Índias: St. Thomas) (gramineas)

Hood, J.D. 1925. New neotropical Thysanoptera collected by C.B. Williams. Psyche, 32(1): 48-69.

Distribuição no Brasil: RJ (Ilha da Gipoia, próximo à Angra dos Reis; Itaguá), SP (Serra da Cantareira, próximo a Franco da Rocha)

Habitat: gramineas, incluindo Andropogon ?condensatus 
Adraneothrips acutus Hood, 1950: 60 (Brasil: Boracéa, municipio de Salesópolis, SP) (Andropogon sp., provavelmente $A$. condensatus)

Hood, J.D. 1950. Brasilian Thysanoptera II. Revista de Entomologia, 21(1-2): 1-113.

Distribuicão no Brasil: SP (Boracéa/Salesópolis)

Habitat: Andropogon sp.

Adraneothrips alternatus Hood, 1925: 55 (Trinidade) (folhas mortas de banana - página superior ao longo da nervura central)

Hood, J.D. 1925. New neotropical Thysanoptera collected by C.B. Williams. Psyche, 32(1): 48-69.

Distribuicão no Brasil: RJ (Illha da Gipoia), SP (Serra da Cantareira/Franco da Rocha, Piracicaba), PA, PR

Habitat: folhas mortas de banana - página superior ao longo da nervura central, ramos motos, serrapilheira, folhas de cenoura

Adraneothrips brasiliensis Hood, 1950: 64 (Brasil: Boracéa, município de Salesópolis, SP) (ramos e folhas mortos)

Hood, J.D. 1950. Brasilian Thysanoptera II. Revista de Entomologia, 21(1-2): 1-113.

Distribuicão no Brasil: SP (Piracicaba, Boracéa/Salesópolis, São Paulo), RJ (Petrópolis, Jacarepaguá/Rio de Janeiro)

Habitat: ramos e folhas mortos; folhas mortas de banana, folhas caidas na sombra, ramo morto

Adraneothrips lepidus Hood, 1950: 87 (Brasil: São Carlos, SP) (graminea)

Hood, J.D. 1950. Brasilian Thysanoptera II. Revista de Entomologia, 21(1-2): 1-113.

Distribuicão no Brasil: SP (São Carlos), RJ (Itaguai)

Habitat: gramíneas

Adraneothrips obliquus Hood, 1950: 71 (Brasil: Jacarepaguá, Rio de Janeiro, RJ) (ramos mortos com folhas) Hood, J.D. 1950. Brasilian Thysanoptera II. Revista de Entomologia, 21(1-2): 1-113.

Distribuiç̃a no Brasil: RJ (Jacarepaguá/Rio de Janeiro)

Habitat: ramos mortos com folhas

Adraneothrips pulchellus Hood, 1950: 83 (Brasil: Fazenda Salto, São Carlos, SP) (vines e arbustos misturados)

Hood, J.D. 1950. Brasilian Thysanoptera II. Revista de Entomologia, 21(1-2): 1-113.

Distribuição no Brasil: SP (São Carlos)

Habitat: vines e arbustos misturados

Adraneothrips septimanus Hood, 1950: 77 (Brasil: Santo Amaro, SP) (Andropogon sp., provavelmente $A$. condensatus)

Hood, J.D. 1950. Brasilian Thysanoptera II. Revista de Entomologia, 21(1-2): 1-113.

Distribuicão no Brasil: SP (Santo Arnaro)

Habitat: Andropogon sp.

Adraneothrips silvaticus Hood, 1950; 68 (Brasil: Jacarepaguá, Rio de Janeiro, RJ) (folhas de banana mortas e dependuradas, em floresta úmida)

Hood, J.D. 1950. Brasilian Thysanoptera II. Revista de Entomologia, 21(1-2): 1-113.

Distribuicão no Brasil: RJ (Jacarepaguá/Rio de Janeiro)

Habitat: folhas de banana mortas e dependuradas

Adraneothrips spadix Hood, 1950: 58 (Brasil: Jacarepaguá, Rio de Janeiro, RJ) (folhas de cana-de-açúcar morta e restos no solo)

Hood, J.D. 1950. Brasilian Thysanoptera II. Revista de Entomologia, 21(1-2): 1-113.

Distribuicão no Brasil: RJ (Jacarepaguá/Rio de Janeiro)

Habitat: folhas mortas de cana-de-açúcar, restos no solo

Adraneothrips stenocephalus Hood, 1938: 362 (EUA: Clearwater, Flórida) (ciperáceas)

Hood, J.D. 1938. New Thysanoptera from Florida and North Carolina. Revista de Entomologia, 8(3-4): 348-420.

Distribuição no Brasil: SP (Fazenda Salto/São Carlos) 
Habitat: Andropogon sp. provavelmente $A$. condensatus

Adraneothrips tibialis (Hood, 1914: 39) (Haplothrips) (Porto Rico: Rio Piedras) (folhas de cana-de-açúcar de internódios pequenos e menos desenvolvidos)

Hood, J.D. 1914. Two Porto Rican Thysanooptera from sugar cane. Insecutor Inscitiae Menstruus, 2(3): 38-41.

Distribuicão no Brasil: RJ (Jacarepaguá/Rio de Janeiro, Itaguaí, Campo Grande

Habitat: bambu, gramíneas, cana-de-açúcar, ramos mortos, folhas de Musa paradisiaca, Piper aduncum (checar todos hosp.)

Adraneothrips transversus Hood, 1950: 74 (Brasil: São Carlos, SP) (ramos mortos)

Hood, J.D. 1950. Brasilian Thysanoptera II. Revista de Entomologia, 21(1-2): 1-113.

Distribuição no Brasil: SP (São Carlos)

Habitat: ramos mortos

Adraneothrips tupi Hood, 1950: 80 (Brasil: Angra dos Reis, RJ) (ramos mortos)

Hood, J.D. 1950. Brasilian Thysanoptera II. Revista de Entomologia, 21(1-2): 1-113.

Distribuicão no Brasil: RJ (Angra dos Reis), SP (Boracéa/Salesópolis, Itanhaém)

Habitat: ramos mortos, graminea

Adraneothrips uniformis Hood, 1925: 56 (Trinidade) (folhas mortas de banana)

Hood, J.D. 1925. New Neotropical Thysanoptera collected by C.B. Williams. Psyche, 32(1): $48-69$

Distribuicão no Brasil: SP (Serra da Cantareira/Franco da Rocha, Fazenda Salto/São Carlos e Itanhaém)

Habitat: ramos mortos no solo; ramos mortos e morrendo de laranja, folhas mortas

Adraneothrips vespicola De Santis, 1981: 1 (Brasil)

De Santis, L. 1981. Descripción de una nueva especies de tisanóptero (Insecta) de Brasil con notas sobre otras dos de la Republica Argentina. Dusenia, 12: 1-3.

Distribuicão no Brasil:

Habitat:

Adraneothrips sp.

Distribuicão no Brasil: SP (Piracicaba, Rio Claro)

Habitat: folhas mortas de Eucalyptus sp.

\section{Gênero Aleurodothrips}

Aleurodothrips fasciapennis (Franklin, 1908: 727) (Cryptothrips) (Barbados: Glendor, St. Michael) (folha de limão)

Franklin, H.J. 1908. On a collection of Thysanopterous insects from Barbados and St. Vicent Islands. Proceedings of the U.S. National Museum, 33: 715-730.

Distribuição no Brasil: SP (Piracicaba)

Habitat: citros

\section{Gênero Amynothrips}

Amynothrips andersoni O’Neill, 1968: 179 (Argentina: Castelar, Provincia de Buenos Aires) (Alternanthera philoxeroides)

O'Neill, K. 1968. Amynothrips andersoni, a new genus and species injurious to alligatorweed (Thysanoptera: Phlaeothripidae). Proceedings of the Entomological Society of Washington, 70(2): 175-183

Distribuicão no Brasil: PA (Belém), AM (Lago Careira, Ilha Careira), PR (Araucaria, próximo a Foz do Rio Iguaçu; localidade próxima à Curitiba, no Rio Iguaçu); RS (Porto Alegre, tha do Pavão) 
Habitat: Alternanthera philoxeroides; Alternanthera hassleriana; introduzido nos Estados Unidos para controle de planta daninha jacaré

\section{Gênero Blepharidothrips}

Blepharidothrips sphaerops Hood, 1952: 149 (Brasil: B oracéa, município de Salesópolis, SP) (ramos secos) Hood, J.D. 1952. Brasilian Thysanoptera III. Proceedings of the Biological Society of Washington, 65: 141-174. Distribuição no Brasil: SP (Boracéa/Salesópolis)

Habitat: ramos secos

\section{Gênero Bradythrips}

Bradythrips hesperus Hood \& Williams, 1925: 68 (em Hood, 1925: 68) (Güiana Britânica: Issoro) (base de Imperata caudata)

Hood, J.D. 1925. New neotropical Thysanoptera collected by C.B. Williams. Psyche, 32(1): 48-69.

Distribuição no Brasil:

Habitat:

\section{Gênero Carathrips}

Carathrips bandeirantium Hood, 1950: 56 (Brasil: São Carlos, SP) (ramos mortos, incluindo os de laranja) Hood, J.D. 1950. Brasilian Thysanoptera II. Revista de Entomologia, 21(1-2): 1-113.

Distribuição no Brasil: SP (São Carlos)

Habitat: ramos mortos, incluindo os de laranja

Carathrips delicatulus (Hood, 1939: 590) (Hoplothrips) (EUA: Pledger, Texas) (sob a casca de ramos mortos) Hood, J.D. 1939. New North American Thysanoptera, principally from Texas. Revista de Entomologia, 10(3): 550619.

Distribuição no Brasil: RJ (Angra dos Reis, Campo Grande, JacarepaguáRio de Janeiro)

Habitat: ramos mortos todos de espécies brasileiras

Carathrips ferrugineus Hood, 1950: 43 (Brasil: Boracéa, Salesópolis, SP) (ramos mortos)

Hood, J.D. 1950. Brasilian Thysanoptera II. Revista de Entomologia, 21(1-2): 1-113.

Distribuicão no Brasil: SP (Boracéa/Salesópolis), RJ (Petrópolis)

Habitat: ramos mortos

Carathrips grandiceps Hood, 1950: 53 (Brasil: Serra da Cantareira, Franco da Rocha, SP) (ramos mortos; algumas gramineas mortas)

Hood, J.D. 1950. Brasilian Thysanoptera II. Revista de Entomologia, 21(1-2): 1-113.

Distribuicão no Brasil: SP (Serra da Cantareira/Franco da Rocha; São Carlos)

Habitat: ramos mortos, gramíneas vivas e mortas

Carathrips impensus Hood, 1956(1955): 137 (Brasil: Rondon, PR) (ramos mortos)

Hood, J.D. 1956(1955). Brasilian Thysanoptera VI. Revista Brasileira de Entomologia, 4: 51-160.

Distribuicão no Brasil: PR (Rondon), SC (Nova Teutônia)

Habitat: ramos mortos

Carathrips interruptus Hood, 1950: 49 (Brasil: São Carlos, SP) (ramos mortos e caídos de várias árvores, incluindo os de laranja e café)

Hood, J.D. 1950. Brasilian Thysanoptera II. Revista de Entomologia, 21(1-2): 1-113.

Distribuicão no Brasil: SP (São Carlos)

Habitat: ramos mortos e caídos de várias árvores, incluindo os de laranja e café, ramos mortos de citros 
Carathrips mediamericanus (Hood, 1933: 412) (Trichothrips) (Panamá: Porto Bello) (ramos mortos de várias espécies)

Hood, J.D. 1933. New Thysanoptera from Panama. Journal of the New York Entomological Society, 41(4): 407-434

Distribuicão no Brasil: RJ (Jacarepaguá/Rio de Janeiro, Itha da Gipóia, Itaguai), SP (São Carlos; Serra da Cantareira/Franco da Rocha)

Habitat: ramos mortos

Carathrips pallidiventris Hood, 1950: 46 (Brasil: Angra dos Reis, RJ) (ramos mortos caídos no chão)

Hood, J.D. 1950. Brasilian Thysanoptera II. Revista de Entomologia, 21(1-2): 1-113.

Distribuição no Brasil: RJ (Angra dos Reis), SP (Boracéa/Salesópolis)

Habitat: ramos mortos caídos no chão

Carathrips plaumanni Hood, 1956(1955): 134 (Brasil: Nova Teutônia, SC) (ramos mortos) Hood, J.D. 1956(1955). Brasilian Thysanoptera VI. Revista Brasileira de Entomologia, 4: 51-160.

Distribuição no Brasil: SC (Nova Teutônia)

Habitat: ramos mortos

Carathrips sp. (citado em Monteiro et al., 1996)

Distribuicão no Brasil: SP (Piracicaba)

Habitat: ramo morto com líquen; ramos de citros vivos com líquen, galhos mortos

\section{Gênero Cartomothrips}

Cartomothrips browni Stannard, 1962: 38 (Austrália: Victoria, McCrea, Arthur's Seat) (dead leafy wattle branches)

Stannard, L.J. 1962. Cartomothrips, a new genus based on two new species from the Australian region (Thysanoptera: Phlaeothripidae). Proceedings of the Royal Entomological Society of London (B) 31: (3-4): 38-40.

Distribuicão no Brasil: SP (Rio Claro, Piracicaba)

Habitat: cápsulas das sementes de Eucalyptus sp., folhas mortas de Eucalyptus sp.

\section{Gênero Chamaeothrips}

Chamaeothrips jucundus Hood, 1954: 44 (Brasil: Nova Teutônia, SC) (folhas caídas)

Hood, J.D. 1954. Brasilian Thysanoptera IV. Proceedings of the Biological Society of Washington, 67: 17-54.

Distribuição no Brasil: SC (Nova Teutônia)

Habitat: folhas caidas

\section{Gênero Chirothripoides}

Chirothripoides typicus Bagnall, 1914: 505 (Trinidade: Verdant Vale, Arima) (desconhecido: coletado em uma janela)

Bagnall, R.S. 1915. On a collection of Thysanoptera from the West Indies, with descriptions of new genera and species. Joumal of the Linnean Society, Zoology, 32(220): 495-507.

Distribuição no Brasil:

Habitat: desconhecido

\section{Gênero Chorithrips}

Chorithrips heptatoma Hood, 1957: 171 (Brasil: Nova Teutônia, SC) (sob folhas caídas)

Hood, J.D. 1957. New Brazilian Thysanoptera. Proceedings of the Biological Society of Washington, 70: 129-180.

Distribuição no Brasil: SC (Nova Teutônia)

Habitat: folhas caidas 
Chorithrips octatoma Hood, 1957: 171 (Brasil: Rio Caraguatá, RS) (folhas caidas)

Hood, J.D. 1957. New Brazilian Thysanoptera. Proceedings of the Biological Society of Washington, 70: 129-180.

Distribuicão no Brasil: RS (Rio Caraguatá)

Habitat: folhas caidas

\section{Gênero Chortothrips}

Chortothrips valens (Hood, 1950: 104) (Eurythrips) (Brasil: Jacarepaguá, Rio de Janeiro, RJ) (gramíneas altas e grossas, incluindo Andropogon condensatus)

Hood, J.D. 1950. Brasilian Thysanoptera II. Revista de Entomologia, 21(1-2): 1-113.

Distribuição no Brasil: RJ (Jacarepaguá/Rio de Janeiro), SP (Itanhaém)

Habitat: gramíneas altas e grossas, incluindo Andropogon condensatus)

\section{Gênero Chthonothrips}

Chthonothrips nigrocinctus Hood, 1957: 143 (Brasil: Nova Teutônia, SC) (folhas caidas)

Hood, J.D. 1957. New Brazilian Thysanoptera. Proceedings of the Biological Society of Washington, 70: 129-180.

Distribuição no Brasil: SC (Nova Teutônia)

Habitat: folhas caidas

\section{Gênero Docessissophothrips}

Docessissophothrips brasiliensis (Schmutz, 1909: 276) (Polyphemothrips) (Brasil: RS) (desconhecido)

Schmutz, K. 1909. Zur Kenntmis einiger neuen Thysanopterengenera (Tubulifera). Annalen des Naturhistorischen Museums, Wien, 23: 273-281.

Distribuicão no Brasil: RS

Habitat: desconhecido

Docessissophothrips dotatus (Hood, 1956(1955): 108) (Polyphemothrips) (Brasil: Belém, PA) (ramos mortos de Hevea sp. caída no chão)

Hood, J.D. 1956(1955). Brasilian Thysanoptera VI. Revista Brasileira de Entomologia, 4: 51-160.

Distribuicão no Brasil: PA (Belém)

Habitat: ramos mortos de Hevea sp. caída no chão

Docessissophothrips tenuiceps (Hood, 1937: 285) (Polyphemothrips) (Peru: vizinhança de Rioja, floresta a cerca de $900 \mathrm{~m}$ de altura, Dept. San Martin) (ramos mortos de árvores caídas no chão há quatro ou cinco meses)

Hood, J.D. 1937. Studies on Neotropical Thysanoptera IV. Revista de Entomologia, 7(2-3): 255-296.

Distribuicão no Brasil:

Habitat:

Docessissophothrips travassosi (Hood, 1949: 55) (Polyphemothrips) (Brasil: Boracéa, Salesópolis, SP) (ramos mortos)

Hood, J.D. 1949. Brasilian Thysanoptera I. Revista de Entomologia, 20(1-3): 3-88.

Distribuicãa no Brasil: SP (Boracéa/Salesópolis), RJ (Petrópolis), SC (Nova Teutônia)

Habitat: ramos mortos

Docessissophothrips villicornis (Hood, 1949: 59) (Polyphemothrips) (Brasil: Jacarepaguá, Rio de Janeiro, $\mathrm{RJ})$ (ramos mortos)

Hood, J.D. 1949. Brasilian Thysanoptera I. Revista de Entomologia, 20(1-3): 3-88.

Distribuição no Brasil: RJ (Jacarepaguá/Rio de Janeiro)

Habitat: ramos mortos; folhas caídas, mortas e secas de manga Mangifera indica 


\section{Gênero Dolichothrips}

\section{Dolichothrips sp.}

Distribuicãa no Brasil: SP (Piracicaba)

Habitat: planta herbácea (folhas e flores), flores de quaresmeira (Tibouchina sp.)

\section{Gênero Epomisothrips}

Epomisothrips araucariae Hood, 1954: 50 (Brasil: Curitiba, PR) (folhas de Araucaria brasiliensis) Hood, J.D. 1954. Brasilian Thysanoptera IV. Proceedings of the Biological Society of Washington, 67: 17-54. Distribuicão no Brasil: PR (Curitiba)

Habitat: folhas e ramos de Araucaria brasiliensis

\section{Gênero Eschatothrips}

Eschatothrips cerinus Hood, 1957: 167 (Brasil: Nova Teutônia, SC) (sob folhas caídas)

Hood, J.D. 1957. New Brazilian Thysanoptera. Proceedings of the Biological Society of Washington, 70: 129-180.

Distribuição no Brasil: SC (Nova Teutônia)

Habitat: sob folhas caídas

Eschatothrips decoratus Hood, 1957: 168 (Brasil: Nova Teutônia, SC) (sob folhas caídas)

Hood, J.D. 1957. New Brazilian Thysanoptera. Proceedings of the Biological Society of Washington, 70: 129-180.

Distribuicão no Brasil: SC (Nova Teutônia)

Habitat: sob folhas caídas

Eschatothrips pachyurus (Hood, 1954: 41) (Orthothrips) (Brasil: Nova Teutônia, SC) (não mencionado)

Hood, J.D. 1954. Brasilian Thysanoptera IV. Proceedings of the Biological Society of Washington, 67: 17-54.

Distribuição no Brasil: SC (Nova Teutônia)

Habitat: desconhecido

Eschatothrips variegatus (Hood, 1954: 41) (Orthothrips) (Brasil: Belém, PA) (folhas mortas de assai Euterpe oleracea)

Hood, J.D. 1954. Brasilian Thysanoptera IV. Proceedings of the Biological Society of Washington, 67: 17-54.

Distribuição no Brasil: PA (Belém)

Habitat: folhas mortas de Euterpe oleracea

\section{Gênero Eupathithrips}

Eupathithrips atripes Hood, 1950: 31 (Brasil: Fazenda Salto, São Carlos, SP) (ramos e folhas mortas) Hood, J.D. 1950. Brasilian Thysanoptera I. Revista de Entomologia, 21(1-2): 1-113.

Distribuicão no Brasil: SP (Fazenda Salto/São Carlos)

Habitat: ramos e folhas mortas

Eupathithrips meizon Hood, 1956(1955): 151 (Brasil: São Paulo, SP) (ramos secos)

Hood, J.D. 1956(1955) Brasilian Thysanoptera VI. Revista Brasileira de Entomologia, 4: 51-160.

Distribuição no Brasil: SP (São Paulo)

Habitat: ramos secos

Eupathithrips silvestrii (Buffa, 1908: 123 ou 124?) (Heterothrips) (Argentina? - em M\&M) (Trinidade ou Paraguai - em Moulton) (em fendas de uma árvore de cacau morta)

Buffa, P. 1908. Tisanotteri nuovi - nota Preliminare. Redia, 4 ou 5?: 123-125: 124.

Distribuição no Brasil: SP (Piracicaba)

Habitat: ramo morto (protegido e recebendo bastante sol) 


\section{Gênero Eurythrips}

Eurythrips alarius Hood, 1957: 134 (Brasil: Nova Teutônia, SC) (não dado)

Hood, J.D. 1957. New Brazilian Thysanoptera. Proceedings of the Biological Society of Washington, 70: 129-180.

Distribuição no Brasil: SC (Nova Teutônia)

Habitat: não mencionado

Eurythrips bifasciatus (Hood, 1954: 37) (Porcothrips) (Brasil: Nova Teutônia, SC) (desconhecido)

Hood, J.D. 1954. Brasilian Thysanoptera IV. Proceedings of the Biological Society of Washington, 67: 17-54.

Distribuição no Brasil: SC (Nova Teutônia)

Habitat: não mencionado

Eurythrips bisetosus (Hood, 1954: 35) (Porcothrips) (Brasil: Nova Teutônia, SC) (desconhecido)

Hood, J.D. 1954. Brasilian Thysanoptera IV. Proceedings of the Biological Society of Washington, 67: 17-54.

Distribuicão no Brasil: SC (Nova Teutônia)

Habitat: desconhecido

Eurythrips braquiaulus Gerdes, 1980: ? (Brasil)

Gerdes, C.F. 1980. Eurythrips and Terthrothrips (Thysanoptera: Phlaeothripidae) from southem Brasil, with one new species, new collection sites, and key. Proceedings of the Entomological Society of Washington, 86(?): 400-410.

Distribuicão no Brasil: SC (Irany)

Habitat:

Eurythrips citricornis (Hood, 1954: 36) (Porcothrips) (Brasil: Nova Teutônia, SC) (não dado)

Hood, J.D. 1954. Brasilian Thysanoptera IV. Proceedings of the Biological Society of Washington, 67: 17-54.

Sinônimo: Euthrips occipitalis Hood, 1957: 139 (Brasil: Nova Teutônia, SC) (sob folhas caidas)

Hood, J.D. 1957. New Brazilian Thysanoptera. Proceedings of the Biological Society of Washington, 70: 129-180.

Distribuicão no Brasil: SC (Nova Teutônia)

Habitat: sob folhas caídas

Eurythrips conformis Hood, 1957: 136 (Brasil: Nova Teutônia, SC) (folhas caidas)

Hood, J.D. 1957. New Brazilian Thysanoptera. Proceedings of the Biological Society of Washington, 70: 129-180.

Distribuicão no Brasil: SC (Nova Teutônia), RS (Erechim)

Habitat: folhas caidas

Eurythrips costalimai Hood, 1950: 99 (Brasil: Jacarepaguá/Rio de Janeiro, RJ) (Andropogon condensatus)

Hood, J.D. 1950. Brasilian Thysanoptera II. Revista de Entomologia, 21(1-2): 1-113.

Distribuicão no Brasil: RJ

Habitat:

Eurythrips cruralis Hood, 1957: 140 (Brasil: Nova Teutônia, SC) (folhas caidas)

Hood, J.D. 1957. New Brazilian Thysanoptera. Proceedings of the Biological Society of Washington, 70: 129-180.

Distribuição no Brasil: SC (Nova Teutônia)

Habitat: folhas caidas

Eurythrips defectus (Hood, 1957: 144) (Plemmelothrips) (Brasil: Nova Teutônia, SC) (sob folhas caidas)

Hood, J.D. 1957. New Brazilian Thysanoptera. Proceedings of the Biological Society of Washington, 70: 129-180.

Distribuicão no Brasil: SC (Nova Teutônia)

Habitat: sob folhas caídas

Eurythrips elongatus Hood, 1957: 140 (Brasil: Nova Teutônia, SC) (sob folhas caídas)

Hood, J.D. 1957. New Brazilian Thysanoptera. Proceedings of the Biological Society of Washington, 70: 129-180.

Distribuicão no Brasil: SC (Nova Teutônia)

Habitat: sob folhas caídas 
Eurythrips hemimeres Hood, 1957: 142 (Brasil: Nova Teutônia, SC) (sob folhas caídas)

Hood, J.D. 1957. New Brazilian Thysanoptera. Proceedings of the Biological Society of Washington, 70: 129-180.

Distribuição no Brasil: SC (Nova Teutônia)

Habitat: sob folhas caídas

Eurythrips modestus (Bagnall, 1917: 24) (Malacothrips) (St. Vicent Island) (gramineas)

Bagnall, R.S. 1917. On a collection of Thysanoptera from St. Vincent, with descriptions of new genera and species. Joumal of Zoological Research, 2(1): 21-27.

Sinônimo: Eurythrips xanthozonus Hood, 1957: 138 (Brasil: Belém, PA) (gramíneas, algumas parcialmente mortas) Hood, J.D. 1957. New Brazilian Thysanoptera. Proceedings of the Biological Society of Washington, 70: 129-180.

Distribuição no Brasil: PA (Belém)

Habitat: gramineas, algumas parcialmente mortas

Eurythrips musivi Hood, 1957: 137 (Brasil: Nova Teutônia, SC) (sob folhas caídas)

Hood, J.D. 1957. New Brazilian Thysanoptera. Proceedings of the Biological Society of Washington, 70: 129-180.

Distribuição no Brasil: SC (Nova Teutônia)

Habitat: sob folhas caídas

Eurythrips nigriceps Hood, 1957: 141 (Brasil: Nova Teutônia, SC) (folhas caídas)

Hood, J.D. 1957. New Brazilian Thysanoptera. Proceedings of the Biological Society of Washington, 70: 129-180.

Distribuicão no Brasil: SC (Nova Teutônia)

Habitat: folhas caídas

Eurythrips nigricornis Hood, 1960: 61 (Brasil) (Nova Teutônia, SC) (gramineas mortas)

Hood, J.D. 1960. Six new Thysanoptera from Brazil. Revista Brasileira de Entomologia, 9: 57-68.

Distribuição no Brasil: SC (Nova Teutônia)

Habitat: gramineas mortas

Eurythrips peccans Hood, 1957: 135 (Brasil: Nova Teutônia, SC) (folhas caídas)

Hood, J.D. 1957. New Brazilian Thysanoptera. Proceedings of the Biological Society of Washington, 70: 129-180.

Distribuicão no Brasil: SC (Nova Teutônia)

Habitat: folhas caídas

Eurythrips phaeomelus Gerdes, 1980: ? (Brasil)

Gerdes, C.F. 1980. Eurythrips and Terthrothrips (Thysanoptera: Phlaeothripidae) from southern Brasil, with one new species, new collection sites, and key. Proceedings of the Entomological Society of Washington, 86(?): 400-410.

Distribuicão no Brasil: SC (Seara)

Habitat:

Eurythrips pusillus Hood, 1957: 135 (Brasil: Nova Teutônia, SC) (folhas caídas)

Hood, J.D. 1957. New Brazilian Thysanoptera. Proceedings of the Biological Society of Washington, 70: 129-180.

Distribuição no Brasil: SC (Nova Teutônia), RS (Erechim)

Habitat: folhas caídas

Eurythrips setosus Hood, 1950: 108 (Brasil: Itaguaí, RJ) (ramos mortos)

Hood, J.D. 1950. Brasilian Thysanoptera II. Revista de Entomologia, 21(1-2): 1-113

Distribuicão no Brasil: RJ (Itaguaí), SP (Itanhaém)

Habitat: ramos mortos, gramineas

Eurythrips simplex (Hood, 1954: 36) (Porcothrips) (Brasil: Nova Teutônia, SC) (desconhecida)

Hood, J.D. 1954. Brasilian Thysanoptera IV. Proceedings of the Biological Society of Washington, 67: 17-54.

Distribuicão no Brasil: SC (Nova Teutônia)

Habitat: desconhecido 
Eurythrips striolatus Hood, 1957: 138 (Brasil: Nova Teutônia, SC) (sob folhas caídas)

Hood, J.D. 1957. New Brazilian Thysanoptera. Proceedings of the Biological Society of Washington, 70: 129-180.

Distribuicão no Brasil: SC (Nova Teutônia)

Habitat: sob folhas caidas

Eurythrips subflavus Hood, 1950: 102 (Brasil: Itaguaí, RJ) (gramineas)

Hood, J.D. 1950. Brasilian Thysanoptera II. Revista de Entomologia, 21(1-2): 1-113

Distribuicão no Brasil: RJ (Itaguaí)

Habitat: gramíneas

Eurythrips tarsalis Hood, 1925: 220 (USA: East Falls Church, Virginia).(base de folhas de Andropogon sp.) Hood, J.D. 1925. Four new Phloeothripidae from the United States (Thysanoptera). Canadian Entomologist, 57(9): 218-222.

Sinônimo: Eurythrips morulops Hood, 1950: 96 (Brasil: Boracéa, Salesópolis, SP) (single clamp of standing Andropogon sp. provavelmente $A$. condensatus)

Hood, J.D. 1950. Brasilian Thysanoptera II. Revista de Entomologia, 21(1-2): 1-113.

Distribuicão no Brasil: SP (Boracéa/Salesópolis; Itanhaém), PA (Belém)

Habitat: gramíneas, incluindo $A$. condensatus

Eurythrips trifasciatus (Hood, 1954: 38) (Porcothrips) (Brasil: Nova Teutônia, SC) (desconhecido)

Hood, J.D. 1954. Brasilian Thysanoptera IV. Proceedings of the Biological Society of Washington, 67: 17-54.

Distribuicão no Brasil: SC (Nova Teutônia)

Habitat: desconhecido

\section{Gênero Glyptothrips}

Glyptothrips bucca (Hood, 1957: 167) (Erkosothrips) (Brasil: Nova Teutônia, SC) (sob folhas caídas) Hood, J.D. 1957. New Brazilian Thysanoptera. Proceedings of the Biological Society of Washington, 70: 129-180. Distribuição no Brasil: SC (Nova Teutônia)

Habitat: sob folhas caídas

Glyptothrips divergens (Hood, 1957: 164) (Orthothrips) (Brasil: Nova Teutônia, SC) (sob folhas caidas) Hood, J.D. 1957. New Brazilian Thysanoptera. Proceedings of the Biological Society of Washington, 70: 129-180. Distribuição no Brasil: SC (Nova Teutônia)

Habitat: sob folhas caídas

Glyptothrips fuscipes (Hood, 1954: 40) (Orthothrips) (Brasil: Nova Teutônia, SC) (desconhecido) Hood, J.D. 1954. Brasilian Thysanoptera IV. Proceedings of the Biological Society of Washington, 67: 17-54.

Distribuicão no Brasil: SC (Nova Teutônia)

Habitat: não mencionado

Glyptothrips hylaeus (Hood, 1950: 111) (Sagenothrips) (Brasil: Serra da Cantareira, Franco da Rocha, SP) (folhas mortas no chão em uma floresta)

Hood, J.D. 1950. Brasilian Thysanoptera II. Revista de Entomologia, 21(1-2): 1-113

Distribuicão no Brasil: SP (Serra da Cantareira/Franco da Rocha), ?SC (Nova Teutônia)

Habitat: sob folhas caídas

Glyptothrips longiceps (Hood, 1954: 39) (Orthothrips) (Brasil: Nova Teutônia, SC) (não dado)

Hood, J.D. 1954. Brasilian Thysanoptera IV. Proceedings of the Biological Society of Washington, 67: 17-54.

Distribuicão no Brasil: SC (Nova Teutônia)

Habitat: não mencionado

Glyptothrips saltuarius (Hood, 1957: 165) (Orthothrips) (Brasil: Nova Teutônia, SC) (sob folhas caídas) Hood, J.D. 1957. New Brazilian Thysanoptera. Proceedings of the Biological Society of Washington, 70: 129-180. Distribuição no Brasil: SC (Nova Teutônia) 
Habitat: sob folhas caidas

Glyptothrips silvaticus (Hood, 1957: 166) (Erkosothrips) (Brasil: Nova Teutônia, SC) (sob folhas caídas) Hood, J.D. 1957. New Brazilian Thysanoptera. Proceedings of the Biological Society of Washington, 70: 129-180. Distribuição no Brasil: SC (Nova Teutônia)

Habitat: sob folhas caídas

Glyptothrips subcalvus (Hood, 1954: 33) (Porcothrips) (Brasil: Nova Teutônia, SC) (desconhecido)

Hood, J.D. 1954. Brasilian Thysanoptera IV. Proceedings of the Biological Society of Washington, 67: 17-54.

Distribuicão no Brasil: SC (Nova Teutônia)

Habitat: desconhecido

\section{Gênero Gomphiothrips}

Gomphiothrips tibouchinae Moulton, 1933: 404 (Brasil: SP) (Tibouchina sp.)

Moulton, D. 1933. The Thysanoptera of South America IV. Revista de Entomologia, 3(3): 385-419.

Distribuição no Brasil: SP (São Paulo)

Habitat: Tibouchina sp.

\section{Gênero Gynaikothrips}

Gynaikothrips ficorum (Marchal, 1908: 252)(Phloeothrips) (Algéria: Alger)

Marchal, P. 1908. Sur une nouvelle espèce de thrips (Thysanoptera) nuisable aux Ficus en Algérie. Bulletin de la Societé Entomologique de France, 14 ou 1908?: 252-253.

Distribuição no Brasil: AM, CE, ES, RJ, PA, RB, PE, RS, SC, SP

Habitat: Ficus sp.

\section{Gênero Haplothrips}

Haplothrips alpester Priesner, 1914: 263

Priesner, 1914. Ent. Zeits.Frankf. a.M., 28: ?-?.

Distribuicão no Brasil: RS (Montenegro)

Habitat: em geral: Compositae, Chrysanthemum spp., Stachys spp., Hieracium spp., Amica montana, Trapogon spp., Bupthalmum spp., Senecio spp. e Carduus spp.

Haplothrips gowdeyi (Franklin, 1908: 725) (Anthothrips) (Barbados)

Franklin, H.J. 1908. On a collection of Thysanopterous insects from Barbados and St. Vicent Islands. Proceedings of the United States Nacional Museum, 33: 715-730.

Distribuição no Brasil: BA, ES, MG, RJ, RS (Santa Cruz do Sul), SP (Santos, Vargem Grande do Sul, Holambra, Piracicaba, Atibaia)

Habitat: algodão, crisântemo, macadâmia,(Macadamia integrifolia), girassol, Hepathorium maximilianae, abobrinha (Cucurbita pepo cv. Caserta), folhas e base da touceira de gramineas

Haplothrips sp. (Trybomiella)

Distribuicão no Brasil: SP (Piracicaba, São Pedro)

Habitat: amendoim, Vernonia sp.

\section{Gênero Holcothrips}

Holcothrips achmaeae Hood, 1954: 51 (Brasil: Rio de Janeiro, RJ) (folhas de Achmaeae sp.)

Hood, J.D. 1954. Brasilian Thysanoptera IV. Proceedings of the Biological Society of Washington, 67: 17-54.

Distribuição no Brasil: RJ (Rio de Janeiro)

Habitat: folhas de Achmaeae sp. 


\section{Gênero Holopothrips}

Holopothrips affinis (Bagnall, 1924: 633) (Phrasterothrips) (Brasil) (desconhecido)

Bagnall, R.S. 1924. Brief description of new Thysanoptera XIV. Annals and Magazine of Natural History, $9^{\text {th }}$ Series, 14(84): 625-640.

Distribuição no Brasil:

Habitat:

Holopothrips ananasi Costa Lima, 1935: 43 (Brasil: RJ) (Ananas sativus)

Costa Lima, A.M. da. 1935. Um novo thysanóptero praga do abacaxi. O Campo, 6(8): 43-44.

Distribuição no Brasil: RJ (Rio de Janeiro, Deodoro)

Habitat: Ananas sp.

Holopothrips balteatus Hood, 1956(1955): 141 (Brasil: Nova Teutônia, SC) (folhas de uma Lauraceae)

Hood, J.D. 1956(1955). Brasilian Thysanoptera VI. Revista Brasileira de Entomologia, 4: 51-160.

Distribuicão no Brasil: SC (Nova Teutônia)

Habitat: folhas de uma Lauraceae

Holopothrips conducans (Priesner, 1921: 210) (Phrasterothrips) (Paraguai: San Bemardim)

Priesner, H. 1921. Neue und wenig bekannte Thysanopteren der neotropischen Fauna aus der Sammlung des Berliner Zoologischen Museums. Deutsche Entomologische Zeitung, 3: 187-223.

Sinônimos:

Phrasterothrips brasiliensis Bagnall, 1924: 632 (Brasil) (sem dados)

Bagnall, R.S. 1924. Brief description of new Thysanoptera XIV. Annals and Magazine of Natural History, $9^{\text {th }}$ Series, 14(84): 625-640.

Diploacantothrips fuscus Moulton, 1933: 240 (Brasil: Santa Theresa, ES) (galhas em Anister sp.)

Moulton, D. 1933. The Thysanoptera of South America III. Revista de Entomologia, 3(2): 227-262.

Distribuição no Brasil: RJ (Manguinhos), ES (Santa Theresa), SP (lbaté)

Habitat: galhas em Myrica sp., galhas em Anister sp., galhas em planta arbustiva não determinada

Holopothrips erianthi (Hood, 1954: 52) (Homorothrips) (Brasil: Piracicaba, SP) (graminea Erianthus asper)

Hood, J.D. 1954. Brasilian Thysanoptera IV. Proceedings of the Biological Society of Washington, 67: 17-54.

Distribuição no Brasil: SP (Piracicaba)

Habitat: Erianthus asper

Holopothrips fulvus Morgan, 1929: 6 (Brasil: BA) (algodão)

Morgan, A.C. 1929. A new genus and five new species of Thysanoptera foreign to the United States. Proceedings of the Entomological Society of Washington, 31(1): 1-9.

Sinônimo: Holopothrips anacardii Hood, 1942: 581 (Brasil: BA) (Anacardium occidentale)

Hood, J.D. 1942. A century of new American Thysanoptera II. Revista de Entomologia, 13(3): 547-678.

Distribuição no Brasil: BA, AM (Manaus) e ?PA

Habitat: algodão, folhas de Caryocar villosum

Holopothrips graminis Hood, 1956(1955): 149 (Brasil: Piracicaba, SP) (graminea Erianthus asper)

Hood, J.D. 1956(1955). Brasilian Thysanoptera VI. Revista Brasileira de Entomologia, 4: 51-160.

Distribuição no Brasil: SP (Piracicaba)

Habitat: gramínea (Erianthus asper)

Holopothrips hambletoni Hood, 1938: 235 (Brasil: SP) (planta não identificada)

Hood, J.D. 1938. Studies in Neotropical Thysanoptera VII. Revista de Entomologia, 9(1-2): 218-247.

Sinônimo: Holopothrips certus Moulton, 1938: 379 (Brasil: MG) (arbusto desconhecido na mata)

Hood, J.D. 1938. Thysanoptera from Minas Geraes, Brazil. Revista de Entomologia, 9(3-4): 374-382.

Distribuição no Brasil: SP, MG

Habitat: planta não identificada, arbusto desconhecido na mata 
Holopothrips hilaris Hood, 1938: 233 (Brasil: SP) (folhagem de uma planta herbácea pequena nas matas) Hood, J.D. 1938. Studies in Neotropical Thysanoptera VII. Revista de Entomologia, 9(1-2): 218-247. Distribuicão no Brasil: SP

Habitat: planta herbácea

Holopothrips inversus Hood, 1956(1955): 146 (Brasil: Itanhaém, SP) (principalmente de vegetação viva misturada)

Hood, J.D. 1956(1955). Brasilian Thysanoptera VI. Revista Brasileira de Entomologia, 4: 51-160.

Distribuicão no Brasil: SP (Itanhaém)

Habitat: vegetação viva misturada

Holopothrips jaboticabae (Hood, 1954: 54) (Anoplothrips) (Brasil: São Carlos, SP) (jaboticaba)

Hood, J.D. 1954. Brasilian Thysanoptera IV. Proceedings of the Biological Society of Washington, 67: 17-54.

Distribuicão no Brasil: SP (São Carlos)

Habitat: jaboticaba

Holopothrips omercooperi (Bagnall, 1924: 633) (Phrasterothrips) (Brasil)

Bagnall, R.S. 1924. Brief description of new Thysanoptera XIV. Annals and Magazine of Natural History, $9^{\text {th }}$ Series, 14(84): 625-640.

Distribuicão no Brasil: sem dados

Habitat: sem dados

Holopothrips pennatus Moulton, 1938: 379 (Brasil: MG) (Apocinaceae lenhosa)

Moulton, D. 1938. Thysanoptera from Minas Geraes, Brazil. Revista de Entomologia, 9(3-4): 374-382.

Sinônimo: Holopothrips arachnionis Hood, 1956(1955): 139 (Brasil: Jacarepaguá, Rio de Janeiro, RJ) (vegetação baixa misturada no jardim)

Hood, J.D. 1956(1955). Brasilian Thysanoptera VI. Revista Brasileira de Entomologia, 4: 51-160.

Distribuição no Brasil: RJ (Jacarepaguá), MG (Viçosa)

Habitat: Apocinaceae lenhosa; vegetação baixa misturada no jardim

Holopothrips pictus Hood, 1941: 584 (Brasil: SP) (folhagem de uma pequena planta herbácea na mata)

Hood, J.D. 1941. A century of new Americxan Thysanoptera III. Revista de Entomologia, 12(3): 547-678.

Distribuição no Brasil: SP

Habitat: folhagem de uma pequena planta herbácea na mata

Holopothrips signatus Hood, 1914: 50 (Tobago Island - ?Panamá) (Hura creptans)

Hood, J.D. 1914. Two new Thysanoptera from Panama. Insecutor Inscitiae Menstruus, 2: 49-53.

Distribuicão no Brasil: MG

Habitat:

Holopothrips tupi Hood, 1956(1955): 143 (Brasil: Serra da Cantareira, Franco da Rocha, SP) (folhas de um arbusto ou árvore não identificado)

Hood, J.D. 1956(1955). Brasilian Thysanoptera VI. Revista Brasileira de Entomologia, 4: 51-160.

Distribuicão no Brasil: SP (Serra da Cantareira/Franco da Rocha)

Habitat: folhas de um arbusto ou árvore não identificado

\section{Gênero Holothrips}

Holothrips aberrans (Hood, 1956(1955): 88) (Adelothrips) (Brasil: Santarém, PA) (ramos mortos)

Hood, J.D. 1956(1955). Brasilian Thysanoptera VI. Revista Brasileira de Entomologia, 4: 51-160.

Distribuicão no Brasil: PA (Santarém)

Habitat: ramos mortos 
Holothrips adelos (Mound, 1968: 146) (Polyphemothrips) (Brasil)

Mound, L.A. 1968. A review of R.S. Bagnall's Thysanoptera collections. Bulletin of the British Museurn (Natural History), Entomology, Supplement II: 1-181.

Sinônimo: Adelothrips caudatus Hood, 1955: 90 (Brasil: Belém, PA) (ramos mortos)

Hood, J.D. 1956(1955). Brasilian Thysanoptera VI. Revista Brasileira de Entomologia, 4: 51-160.

Distribuição no Brasil: PA (Belém)

Habitat: ramos mortos

Holothrips amplus Hood, 1952: 160 (Brasil: Nova Teutônia, SC) (ramos secos)

Hood, J.D. 1952. Brasilian Thysanoptera III. Proceedings of the Biological Society of Washington, 65: 141-174.

Distribuicão no Brasil: SC (Nova Teutônia)

Habitat: ramos secos

Holothrips aspericaudus Hood, 1952: 161 (Brasil: Boracéa, Salesópolis, SP) (ramos mortos)

Hood, J.D. 1952. Brasilian Thysanoptera III. Proceedings of the Biological Society of Washington, 65: 141-174.

Distribuicão no Brasil: SP (Boracéa/Salesópolis)

Habitat: ramos mortos

Holothrips bellulus (Hood, 1956(1955): 92) (Adelothrips) (Brasil: Belém, PA) (folhas mortas de Euterpe oleracea)

Hood, J.D. 1956(1955). Brasilian Thysanoptera VI. Revista Brasileira de Entomologia, 4: 51-160.

Distribuicão no Brasil: PA (Belém)

Habitat: folhas mortas de Euterpe oleracea

Holothrips bucallis (Hood, 1956(1955): 94) (Adelothrips) (Brasil: Belém, PA) (ramos mortos de Hevea sp.)

Hood, J.D. 1956(1955). Brasilian Thysanoptera VI. Revista Brasileira de Entomologia, 4: 51-160.

Distribuição no Brasil: PA (Belém)

Habitat: ramos mortos de Hevea sp.

Holothrips bursarius (Hood, 1957: 174) (Polyphemothrips) (Brasil: Rondon, PR) (ramos mortos)

Hood, J.D. 1957. New Brazilian Thysanoptera. Proceedings of the Biological Society of Washington, 70: 129-180.

Distribuição no Brasil: PR (Rondon)

Habitat: ramos mortos

Holothrips conicurus (Hood, 1942: 611) (Adelothrips) (Peru: Roja (Rio Negro), Dept. San Martin) (ramos e folhas mortas)

Hood, J.D. 1942. A century of new American Thysanoptera III. Revista de Entomologia, 13: 547-678.

Distribuição no Brasil: PA (Belém)

Habitat: ramo morto de Hevea sp.

Holothrips cornutus (Hood, 1956(1955): 96) (Adelothrips) (Brasil: Nova Teutônia, SC) (ramos mortos)

Hood, J.D. 1956(1955). Brasilian Thysanoptera VI. Revista Brasileira de Entomologia, 4: 51-160.

Distribuição no Brasil: SC (Nova Teutônia)

Habitat: ramos mortos

Holothrips eucharis (Hood, 1956(1955): 84) (Adelothrips) (Brasil: Fordlândia, Rio Tapajós, PA) (gramineas mortas)

Hood, J.D. 1956(1955). Brasilian Thysanoptera VI. Revista Brasileira de Entomologia, 4: 51-160.

Distribuicão no Brasil: PA (Fordlândia/Rio Tapajós)

Habitat: gramineas mortas

Holothrips formosus (Hood, 1952: 158) (Adelothrips) (Brasil: Jacarepaguá, Rio de Janeiro, RJ) (ramos mortos) Hood, J.D. 1952. Brasilian Thysanoptera III. Proceedings of the Biological Society of Washington, 65: 141-174.

Distribuicão no Brasil: RJ (Jacarepaguá/Rio de Janeiro, Ilha da Gipoia)

Habitat: ramos mortos 
Holothrips graminicolus (Hood, 1952: 157) (Adelothrips) (Brasil: Jacarepaguá, Rio de Janeiro, RJ) (Andropogon sp. e outras gramineas)

Hood, J.D. 1952. Brasilian Thysanoptera III. Proceedings of the Biological Society of Washington, 65: 141-174.

Distribuição no Brasil: RJ (JacarepaguáRio de Janeiro)

Habitat: Andropogon sp. e outras gramíneas

Holothrips ingens Karny, 1911: 502 (Paraguai) (desconhecido)

Karny, H. 1911. Neue Phloeothripiden-Genera. Zoologischen Anzeiger, 38(22/23): 501-504.

Sinônimo: Adelothrips fenestralis Hood, 1949: 67 (Brasil: São Carlos, SP) (ramos mortos)

Distribuição no Brasil: SP (São Carlos, Boracéa/Salesópolis), RJ (Jacarepaguá/Rio de Janeiro, Ilha da Gipoia)

Habitat: ramos mortos

Holothrips lanei (Hood, 1949: 63) (Adelothrips) (Brasil: Boracéa, Salesópolis) (ramos mortos)

Hood, J.D. 1949. Brasilian Thysanoptera I. Revista de Entomologia, 20(1-3): 3-88.

Distribuição no Brasil: SP (Boracéa/Salesópolis, São Carlos)

Habitat: ramos mortos

Holothrips palmarum (Hood, 1952: 157) (Adelothrips) (Brasil: Belém, PA) (Euterpe sp. e Astrocaryum sp.) Hood, J.D. 1952. Brasilian Thysanoptera III. Proceedings of the Biological Society of Washington, 65: 141-174.

Distribuição no Brasil: PA (Belém)

Habitat: Euterpe sp., Astrocaryum sp.

Holothrips procerus Hood, 1952: 160 (Brasil: Boracéa, Salesópolis, SP) (ramos mortos)

Hood, J.D. 1952. Brasilian Thysanoptera III. Proceedings of the Biological Society of Washington, 65: 141-174.

Distribuição no Brasil: SP (Boracéa/Salesópolis)

Habitat: ramos mortos

Holothrips umbricola (Hood, 1952: 159) (Adelothrips) (Brasil: Boracéa/Salesópolis, SP) (ramos mortos em floresta densa e úmida)

Hood, J.D. 1952. Brasilian Thysanoptera III. Proceedings of the Biological Society of Washington, 65: 141-174.

Distribuicão no Brasil: SP (Boracéa/Salesópolis), RJ (Petrópolis)

Habitat: ramos mortos em floresta densa e úmida, ramos mortos na sombra

\section{Holothrips sp.}

Distribuição no Brasil:

Habitat:

\section{Gênero Hoplandrothrips}

Hoplandrothrips affinis Hood, 1915: 241 (Guiana Inglesa: Berbice) (entre bainha das folhas "leaf sheats"de cana-de-açúcar)

Hood, J.D. 1915. A new Hoplandothrips (Thysanoptera) from British Guiana. Canadian Entomologist, 47: 241-244.

Distribuicão no Brasil: SP (São Carlos), RJ, MG

Habitat: gramíneas, folhagem de arroz

Hoplandrothrips albipes Hood, 1952: 152 (Brasil: Jacarepaguá, Rio de Janeiro, RJ) (ramo e folhas mortas) Hood, J.D. 1952. Brasilian Thysanoptera III. Proceedings of the Biological Society of Washington, 65: 141-174.

Distribuição no Brasil: RJ (Jacarepaguá/Rio de Janeiro)

Habitat: ramos e folhas mortas

Hoplandrothrips brasiliensis Hood, 1954: 45 (Brasil: Iha da Gipoia, perto de Angra dos Reis, RJ) (ramos mortos)

Hood, J.D. 1954. Brasilian Thysanoptera IV. Proceedings of the Biological Society of Washington, 67: 17-54.

Distribuicão no Brasil: RJ (Tha da Gipoia, Jacarepaguá/Rio de Janeiro)

Habitat: ramos mortas, folha morta e dependurada de Dahlia sp. 
Hoplandrothrips erythrinae (Priesner, 1925: 27) (Phloeothrips) (Suriname: Paramaribo) (Erythrina glauca) Priesner, H. 1925. Neue Thysanopteren. Deutsche Entomologische Zeitung, 1925?: 13-28.

Distribuicão no Brasil: RJ e PR

Habitat:

Hoplandrothrips flavipes Bagnall, 1923: 628 (África - Kenya: Kijalie)

Bagnall, RS. 1923. Brief descriptions of new Thysanoptera. XIII. Annals and Magazine of Natural History, $9^{\text {th }}$ Series, 12(71): 624-631.

Distribuição no Brasil: PA (Belém)

Habitat: vine morta sobre uma graminea alta

Hoplandrothrips fusciflavus Hood, 1952: 151 (Brasil: Iha da Gipoia, RJ) (ramos mortos)

Hood, J.D. 1952. Brasilian Thysanoptera III. Proceedings of the Biological Society of Washington, 65: 141-174.

Distribuicão no Brasil: RJ (Tha da Gipoia, Jacarepaguá/Rio de Janeiro), SP (São Carlos)

Habitat: ramos mortos

Hoplandrothrips longirostris Hood, 1954: 46 (Brasil: Petrópolis, RJ) (ramos mortos)

Hood, J.D. 1954. Brasilian Thysanoptera IV. Proceedings of the Biological Society of Washington, 67: 17-54.

Distribuicão no Brasil: RJ (Petrópolis), SP (Boracéa/Salesópolis)

Habitat: ramos mortos

Hoplandrothrips ommatus Hood, 1952: 154 (Brasil: Jacarepaguá, Rio de Janeiro, RJ) (gramíneas e uma "sedge")

Hood, J.D. 1952. Brasilian Thysanoptera III. Proceedings of the Biological Society of Washington, 65: 141-174.

Distribuicão no Brasil: RJ (Jacarepaguá/Rio de Janeiro)

Habitat: gramineas e uma "sedge"

Hoplandrothrips sides (Moulton, 1933: 396) (Phloeothrips (Hoplandrothrips)) (Brasil: BA) (Psidium sp, romã)

Moulton, J.D. 1933. The Thysanoptera of South America IV. Revista de Entomologia, 3(3): 385-419.

Distribuicão no Brasil: BA

Habitat: Psidium sp., romã

Hoplandrothrips variegatus Hood, 1952: 153 (Brasil: Boracéa, Salesópolis, SP) (ramos mortos)

Hood, J.D. 1952. Brasilian Thysanoptera II. Proceedings of the Biological Society of Washington, 65: 141-174.

Distribuição no Brasil: SP (Boracéa/Salesópolis), RJ (Jacarepaguá, Rio de Janeiro, RJ)

Habitat: ramos mortos

Hoplandrothrips xanthopoides Bagnall, 1917: 22 (St. Vicent) (sem dado)

Bagnall, RS. 1917. On a collection of Thysanoptera from St. Vicent, with description of four new species. Journal of Zoological Research, 2: 21-27.

Distribuicão no Brasil: PA, RJ, SP

Habitat:

Hoplandrothrips sp.

Distribuição no Brasil: SC

Habitat:

\section{Gênero Hoplothrips}

Hoplothrips agrestis Hood, 1956(1955): 126 (Brasil: Belém, PA) (Andropogon sp. com vegetação viva e morta misturada)

Hood, J.D. 1956(1955). Brasilian Thysanoptera VI. Revista Brasileira de Entomologia, 4: 51-160.

Distribuição no Brasil: PA (Belém)

Habitat: Andropogon sp. com vegetação viva e morta misturada 
Hoplothrips bahiaensis Moulton, 1933: 235 (Brasil: BA) (algodão)

Moulton, D. 1933. The Thysanoptera of South America III. Revista de Entomologia, 3(3): 385-419.

Distribuicão no Brasil: BA

Habitat: algodão

Hoplothrips dentiger Hood, 1949: 40 (Brasil: Boracéa, Salesópolis, SP) (ramos mortos)

Hood, J.D. 1949. Brasilian Thysanoptera I. Revista de Entomologia, 20(1-3): 3-88.

Distribuicão no Brasil: SP (Boracéa/Salesópolis, Piracicaba)

Habitat: ramos mortos, ramos vivos de citros com líquen

Hoplothrips detector Hood, 1956(1955): 131 (Brasil: São Paulo, SP) (ramos mortos)

Hood, J.D. 1956(1955). Brasilian Thysanoptera VI. Revista Brasileira de Entomologia, 4: 51-160.

Distribuicãa no Brasil: SP (Sào Paulo)

Habitat: ramos mortos

Hoplothrips dissonus Hood, 1956(1955): 129 (Brasil: Boracéa, Salesópolis, SP) (sob casca de árvore em decomposição)

Hood, J.D. 1956(1955). Brasilian Thysanoptera VI. Revista Bvrasileira de Entomologia, 4: 51-160.

Distribuição no Brasil: SP (Boracéa/Salesópolis)

Habitat: sob a casca de árvore em decomposição

Hoplothrips lacteus Hood, 1954: 21 (Brasil: Boracéa, Salesópolis, SP) (ramos mortos na borda de uma floresta úmida)

Hood, J.D. 1954. Brasilian Thysanoptera IV. Proceedings of the Biological Society of Washington, 67: 17-54.

Distribuição no Brasil: SP (Boracéa/Salesópolis)

Habitat: ramos mortos

Hoplothrips orbiculatus Hood, 1954: 22 (Brasil: Serra da Cantareira, Franco da Rocha, SP) (ramos mortos no chão)

Hood, J.D. 1954. Brasilian Thysanoptera IV. Proceedings of the Biological Society of Washington, 67: 17-54.

Distribuição no Brasil: SP (Serra da Cantareira/Salesópolis)

Habitat: ramos mortos no chão

Hoplothrips palmarius Hood, 1956(1955): 124 (Brasil: Belém, PA) (folhas mortas de Bactris maraja)

Hood, J.D. 1956(1955). Brasilian Thysanoptera VI. Revista Brasileira de Entomologia, 4: 51-160.

Distribuicão no Brasil: PA (Belém)

Habitat: folhas mortas de Bactris maraja; folhas mortas de Euterpe oleracea

Hoplothrips psidii Moulton, 1933: 236 (Brasil: BA) (Psidium sp.)

Moulton, D. 1933. The Thysanoptera of South America II. Revista de Entomologia, 3(2): 227-262.

Sinônimo: Plecothrips bruneri Watson, 1933: 18 (Brasil: BA)

Watson, J.L. Two new species of Plectothrips. Florida Entomologist, 17: 16-18 \& 33-34.

Distribuição no Brasil: BA

Habitat: Psidium sp.

Hoplothrips spissicornis Hood, 1952: 148 (Brasil: Belém, PA) (ramos mortos de Hevea sp.)

Hood, J.D. 1952. Brasilian Thysanoptera III. Proceedings of the Biological Society of Washington, 65: 141-174.

Distribuicão no Brasil: PA (Belém)

Habitat: ramos mortos de Hevea sp.

Hoplothrips testaceus Hood, 1954: 21 (Brasil: Nova Teutônia, SC) (ramos mortos)

Hood, J.D. 1954. Brasilian Thysanoptera IV. Proceedings of the Biological Society of Washington, 67: 17-54.

Distribuicão no Brasil: SC (Nova Teutônia)

Habitat: ramos mortos 


\section{Hoplothrips sp.}

Distribuição no Brasil:

Habitat:

\section{Gênero Hyidiothrips}

Hyidiothrips nanellus Hood, 1957: 180 (Brasil: Nova Teutônia, SC) (ramos mortos) Hood, J.D. 1957. New Brazilian Thysanoptera. Proceedings of the Biological Society of Washington, 70: 129-180. Distribuição no Brasil: SC (Nova Teutônia)

Habitat: ramos mortos

Hyidiothrips tesselatus Hood, 1952: 174 (Brasil: Nova Teutônia, SC) (Solanum sp.)

Hood, J.D. 1952. Brasilian Thysanoptera III. Proceedings of the Biological Society of Washington, 65: 141-174.

Distribuicão no Brasil: SC (Nova Teutônia)

Habitat: Solanum sp.

\section{Gênero Karnyothrips}

Karnyothrips flavipes (Jones, 1912: 18) (Anthothrips) (EUA: Califörnia)

Jones, P.R 1912. Some new Califomia and Georgia Thysanoptera. U.S.D.A. Bureau of Entomology, Technical Series 23: 1-24.

Distribuição no Brasil: SP (Fazenda Ribeirão Jaguariúna), RJ, MG (Carmo do Rio Claro)

Habitat: Citrus sp.

Karnyothrips franciscanus (Hood, 1949: 26) (Watsoniella) (Brasil: Petrópolis, RJ) (ramos mortos e folhas mortas, incluindo as de bambu)

Hood, J.D. 1949. Brasilian Thysanoptera I. Revista de Entomologia, 20(1-3): 3-88.

Distribuição no Brasil: RJ (Petrópolis), SP (Boracéa/Salesópolis)

Habitat: ramos e folhas mortas, incluindo os de bambu

Karnyothrips melaleucus (Bagnall, 1911: 61) (Hindsiana) (Dinamarca: Copenhague) (flor de uma Cruciferae em casa-de-vegetação)

Bagnall, RS. 1911. Descriptions of three new Scandinavian Thysanoptera (Tubulifera) Entomologist's Montly Magazine, 47: 60-63.

Distribuiç̃a no Brasil: MG, SP (Piracicaba), RJ

Habitat: galhos mortos

Karnyothrips merrilli (Watson, 1920: 7, 12) (Haplothrips)

Watson, J.L. 1920. An apparently new Haplothrips from Cuba. Florida Entomologist, 4: 7,12.

Sinônimo: Kamyothrips tenuis Moulton, 1938: 381 (Brasil: MG) (galhos mortos de mandioca)

Moulton, D. 1938. Thysanoptera from Minas Geraes, Brazil. Revista de Entomologia, 9(3-4): 374-382.

Distribuição no Brasil: MG (Viçosa)

Habitat:

Karnyothrips nigriflavus (Ramałishna)?

Referência?

Distribuição no Brasil: RJ (Petrópolis)

Habitat: bambu morto e morrendo, ramos de várias árvores

Karnyothrips venustus (Moulton, 1941: 319) (Cryptothrips ou Cephalothrips) (Brasil: MG) (folhagem de citros)

Moulton, D. 1941. Thysanoptera from Minas Geraes, Brazil (second paper). Revista de Entomologia, 12(1-2): 314 322.

Distribuição no Brasil: MG, SP (Piracicaba) 
Habitat: folhagem de citros, haste morta de mandioca, madeira morta com líquen, ramo de citros vivo com líquen, ramos e galhos mortos

\section{Karnyothrips sp.}

Distribuição no Brasil: SP (Rafard, Piracicaba)

Habitat: pimentão, ramo morto (protegido e recebendo bastante sol)

\section{Gênero Leptothrips}

Leptothrips mali (Fitch, 1855 ou 1854?: 808 ou 806?) (Phloeothrips) (EUA: New York)

Fitch, A. 1855. The apple thrips, Phloeothrips mali. Report on the noxious, beneficial, and other insects of the state of New York. Transactions of the New York State Agriculture Society, 14: 806-808.

Distribuição no Brasil:

Habitat:

Leptothrips tenuiceps Hood, 1950: 93 (Brasil: Fazenda Salto, São Carlos, SP) (arbustos e laranjeira)

Hood, J.D. 1950. Brazilian Thysanoptera II. Revista Brasileira de Entomologia, 21(1-2): 1-113.

Distribuicão no Brasil: SP (Fazenda Salto, São Carlos)

Habitat: arbustos e laranjeira

Leptothrips vittipennis Hood, 1938: 221 (Panamá: Juan Diaz) (arbusto Compositae)

Hood, J.D. 1938. Studies in Neotropical Thysanoptera VII. Revita de Entomologia, 9(1-2): 218-247.

Distribuição no Brasil: RJ (Jardim do "Lar das Cegas", Jacarepaguá/Rio de Janeiro, tha Gipoia/próximo à Angra dos Reis), SP

Habitat: vegetação misturada no jardim, gramíneas (Eleusine indica)

\section{Leptothrips sp.}

Distribuição no Brasil: SP (São Pedro)

Habitat: Vernonia sp.

\section{Gênero Liothrips}

Liothrips adisi zur Strassen, 1978: 551 (Brasil) (folhas, flores e brotos novos de guaraná)

zur Strassen, R. 1978. A new species of Liothrips from the Amazonas harmful to guarana plantations (Insecta: Thysanoptera: Phlaeothripidae). Senckenbergiana Biologica, 61: 47-56.

Distribuição no Brasil: AM (Manaus)

Habitat: folhas jovens de guaraná (Paullinia cupana)

Liothrips anonae Moulton, 1933: 248 (Brasil: BA) (Anona squamosa)

Moulton, D. 1933. The Thysanoptera of South America III. Revista de Entomologia, 3(2): 227-262.

Distribuicão no Brasil: BA

Habitat: Myrtus sp.

Liothrips bondari Moulton, 1933: 247 (Brasil: BA) (Myrtus sp.)

Moulton, D. 1933. The Thysanoptera of South America III. Revista de Entomologia, 3(2): 227-262.

Distribuicão no Brasil: BA

Habitat:

Liothrips brasiliensis Moulton, 1933: 255 (Brasil: Colônia Rio Bonito, ES) (um legume)

Moulton, D. 1933. The Thysanoptera of South America III. Revista de Entomologia, 3(2): 227-262.

Distribuição no Brasil: ES (Colônia Rio Bonito), MG

Habitat: um legume 
Liothrips capnodes (Hood, 1956(1955): 106) (Rhyncothrips) (Brasil: Boracéa, município de Salesópolis, SP) (ramos mortos)

Hood, J.D. 1956(1955). Brasilian Thysanoptera VI. Revista Brasileira de Entomologia, 4: 51-160.

Distribuicão no Brasil: SP (Boracéa/Salesópolis)

Habitat: ramos mortos

Liothrips condei Moulton, 1933: 250 (Brasil: Santa Thereza, ES) (Serjania sp. ou Paullinia sp.)

Moulton, D. 1933. The Thysanoptera of South America III. Revista de Entomologia, 3(2): 227-262.

Distribuicão no Brasil: ES (Santa Thereza)

Habitat:

Liothrips didymopanicis Del Claro \& Mound, 1996 (Brasil: Uberlândia, MG) (Didymoparax vinosum)

Del-Claro, K. \& L.A. Mound. 1996. Phenology and description of a new species of Liothrips (Thysanoptera: Phlaeothripidae) from Didymopanax (Araliaceae) in Brazilian cerrado. Revista de Biologia Tropical, 44: 193-197.

Distribuicão no Brasil: MG (Uberlândia)

Habitat: Didymoparax vinosum

Liothrips distinctus Moulton, 1938: 380 (Brasil: MG) (arbusto desconhecido na mata)

Moulton, D. 1938. Thysanoptera from Minas Geraes, Brazil. Revista de Entomologia, 9(3-4): 374-382.

Distribuição no Brasil: MG

Habitat: arbusto desconhecido na mata

Liothrips errabundus zur Strassen, 1975: 82 (Brasil)

zur Strassen, R 1975. Thysanopterogische Notizen (3). Senckenbergiana Biologica, 56: 75-88.

Sinônimo: Liothrips claripennis Moulton, 1933: 253 (Brasil: Afonso Cláudio, ES) (coletando com rede)

Distribuição no Brasil: ES

Habitat:

Liothrips mendesi Moulton, 1933: 251 (Brasil: Monte Itatyaia, RJ) (não determinado)

Moulton, D. 1933. The Thysanoptera of South America III. Revista de Entomologia, 3(2): 227-262.

Distribuição no Brasil: RJ (Itatiaya)

Habitat: sem dados

Liothrips nigriculus (Hood, 1956(1955): 104) (Rhyncothrips) (Brasil: Boracéa, municipio de Salesópolis, SP) (ramos mortos)

Hood, J.D. 1956(1955). Brasilian Thysanoptera VI. Revista Brasileira de Entomologia, 4: 51-160.

Distribuição no Brasil: SP (Boracéa/Salesópolis)

Habitat: ramos mortos

Liothrips salti Moulton, 1933: 256 (Colômbia: Sevilla) (folha de banana)

Moulton, D. 1933. The Thysanoptera of South America III. Revista de Entomologia, 3(2): 227-262.

Distribuicão no Brasil:

Habitat:

Liothrips satanas (Priesner, 1923: 105) (Suriname: Paramaribo) (galhas de uma determinada planta)

Priesner, H. 1923. Ein Beitrag zur Kenntnis der ThysanopterenSurinams. Tijdschrift voor Entomologie, 66: 88-111. Distribuição no Brasil: AM (Manaus)

Habitat: folhas jovens de guaraná (Paullinia cupana)

Liothrips seticollis Kamy, 1912: 471 (Paraguai) (desconhecido)

Karny, H. 1912. On the genera Liothrips and Hoodia. Transactions of the Entomological Society of London, 60(2): 470-475.

Distribuicão no Brasil: MG

Habitat: 
Liothrips tropicus (Schmutz, 1909: 342) (Smerintothrips) (Brasil) (inflorescência)

Schmutz, K. 1909. Zur Kenntmis einiger neuen Thysanopterengenera (Tubulifera). Annalen des Naturhistorischen Museums, Wien, 23: 342-347.

Distribuição no Brasil: SC (Blumenau)

Habitat: sem dados

Liothrips unicolor Moulton, 1933: 254 (Brasil: ES) (desconhecido)

Moulton, D. 1933. The Thysanoptera of South America III. Revista de Entomologia, 3(2): 227-262.

Distribuicão no Brasil: ES

Habitat: desconhecido

Liothrips vernoniae Moulton, 1933: 249 (Brasil: Santa Thereza, ES) (Vernonia sp.)

Moulton, D. 1933. The Thysanoptera of South America III. Revista de Entomologia, 3(2): 227-262.

Distribuicão no Brasil: ES (Santa Thereza), RJ (São Bento), SP, RCM 39

Habitat: Vernonia sp., Compositae

\section{Liothrips $\mathbf{s p}_{1}$.}

Distribuicão no Brasil: AM (Lago Janauca)

Habitat: pyrethrum fogging

\section{Liothrips $\mathbf{s p}_{2}$.}

Distribuição no Brasil: SP (Piracicaba)

Habitat: citros (Citrus sp.), folhas retorcidas de goiaba, galhos mortos

\section{Gênero Lissothrips}

Lissothrips dispar Hood, 1954: 23 (Brasil: Belém, PA) (musgo em árvore de Hevea sp.)

Hood, J.D. 1954. Brasilian Thysanoptera IV. Proceedings of the Biological Society of Washington, 67: 17-54.

Distribuição no Brasil: PA (Belém)

Habitat: musgo em Hevea sp.

Lissothrips eburifer Hood, 1954: 23 (Brasil: Nova Teutônia, SC) (ramos mortos)

Hood, J.D. 1954. Brasilian Thysanoptera IV. Proceedings of the Biological Society of Washington, 67: 17-54.

Distribuição no Brasil: SC

Habitat: ramos mortos

Lissothrips flavidus Hood, 1960: 65 (Brasil: Nova Teutônia, SC) (litter from primeval forest)

Hood, J.D. 1960. Six new Thysanoptera from Brazil. Revista Brasileira de Entomologia, 9: 57-68.

Distribuição no Brasil: SC (Nova Teutônia)

Habitat: litter from primeval forest

Lissothrips obesus Hood, 1950: 38 (Brasil: Boracéa, Salesópolis, SP) (ramos mortos próximos a beirada de uma floresta úmida chuvosa)

Hood, J.D. 1950. Brasilian Thysanoptera II. Revista de Entomologia, 21(1-2): 1-113.

Distribuição no Brasil:

Habitat:

\section{Gênero Lonchothrips}

Lonchothrips linearis Hood, 1957: 177 (Brasil: Rio Caraguatá, Mato Grosso do Sul) (ramos mortos) Hood, J.D. 1957. New Brazilian Thysanoptera. Proceedings of the Biological Society of Washington, 70: 129-180.

Distribuição no Brasil: MS (Rio Caraguatá)

Habitat: ramos mortos 


\section{Gênero Macrophthalmothrips}

Macrophthalmothrips allops Hood, 1954: 47 (Brasil: Belém, PA) (ramos mortos, incluindo alguns de Hevea sp.)

Hood, J.D. 1954. Brasilian Thysanoptera IV. Proceedings of the Biological Society of Washington, 67: 17-54.

Distribuição no Brasil: PA

Habitat: ramos mortos, incluindo os de Hevea sp.

Macrophthalmothrips diasi Hood, 1949: 50 (Brasil: São Carlos, SP) (ramos mortos de laranja, café e outros) Distribuicão no Brasil: SP (São Carlos)

Habitat: ramos mortos de laranja, café e outros

Macrophthalmothrips femoralis Hood, 1941: 605 (Peru: Vizinhança de Celendin, Dept. Cajamarca) (arbusto frequentemente contendo ramos secos com musgo)

Hood, J.D. 1941. A century of new American Thysanoptera III. Revista de Entomologia, 12(3): 547-678.

Distribuição no Brasil:

Habitat:

Macrophthalmothrips hemipteroides (Priesner, 1921: 215) (Ophthalmothrips) (Paraguai) (desconhecido)

Priesner, H. 1921. Neue und Wenig bekannte Thysanopteren der neotropischen Fauna aus der Sammlung des Berliner Zoologischen Museums. Deutsche Entomologische Zeitschrift (Berliner entomologische Zeitschrift und Deutsche Entomologische Zeitschrift in Wiedervereinigung) 3: 187-223.

Distribuicão no Brasil: PR (Rondon), SC (Nova Teutônia), SP (Piracicaba)

Habitat: ramo morto

\section{Gênero Malacothrips}

Malacothrips fasciatus Hood, 1952: 150 (Brasil: Nova Teutônia, SC) (gramineas)

Hood, J.D. 1952. Brasilian Thysanoptera III. Proceedings of the Biological Society of Washington, 65: 141-174.

Distribuição no Brasil: SC (Nova Teutônia), SP (Piracicaba)

Habitat: base da touceira de gramineas

Malacothrips mediater Hood, 1952: 151 (Brasil: Nova Teutônia, SC) (gramineas)

Hood, J.D. 1952. Brasilian Thysanoptera III. Proceedings of the Biological Society of Washington, 65: 141-174.

Distribuição no Brasil: SC (Nova Teutônia)

Habitat: gramineas

Malacothrips vigilatus (Hood, 1957: 132) (Udeothrips) (Brasil: Rio Caraguatá, MS) (folhas caidas)

Hood, J.D. 1957. New Brazilian Thysanoptera. Proceedings of the Biological Society of Washington, 70: 129-180.

Distribuicão no Brasil: MS (Rio Caraguatá), BA

Habitat: folhas caidas

\section{Gênero Menothrips}

Menothrips ebriosus Hood, 1957: 175 (Brasil: Rio Caraguatá, MS) (ramos mortos)

Hood, J.D. 1957. New Brazilian Thysanoptera. Proceedings of the Biological Society of Washington, 70: 129-180. Distribuição no Brasil: Mato Grosso (Rio Caraguatá)

Habitat: ramos mortos

\section{Gênero Mystrothrips}

Mystrothrips clavatoris Hood, 1954: 32 (Brasil: Nova Teutônia, SC) (sem dado) Hood, J.D. 1954. Brasilian Thysanoptera IV. Proceedings of the Biological Society of Washington, 67: 17-54. Distribuicão no Brasil: SC (Nova Teutônia) 
Habitat: sem dado

\section{Gênero Neoheegeria}

Neoheegeria dalmatica Schmutz, 1909: 344 (Europa: Dalmatia) (Phlomis fruticosa)

Schmutz, K. 1909. Zur Kenntnis einiger neuen Thysanopterengenera (Tubulifera). Annalen des Naturhistorischen Museums, Wien, 23: 342-347.

Distribuicão no Brasil: RS (Montenegro)

Habitat:?

\section{Gênero Neurothrips}

Neurothrips frontalis Hood, 1952: 155 (Brasil: Boracéa, Salesópolis, SP) (ramos mortos)

Hood, J.D. 1952. Brasilian Thysanoptera II. Proceedings of the Biological Society of Washington, 65: 141-174.

Distribuição no Brasil: SP (Boracéa/Salesópolis, Piracicaba, Rio Claro)

Habitat: ramos mortos, galho morto, gramineas, folhas mortas de Eucalyptus sp.

Neurothrips n. sp.

Distribuicão no Brasil: SP (Piracicaba, Rio Claro)

Habitat: galho morto, gramineas, folhas mortas de Eucalyptus sp.

\section{Gênero Orthothrips}

Orthothrips angustus Hood, 1954: 39 (Brasil: Nova Teutônia, SC) (não dado)

Hood, J.D. 1954. Brasilian Thysanoptera IV. Proceedings of the Biological Society of Washington, 67: 17-54.

Distribuicão no Brasil: SC (Nova Teutônia)

Habitat: não dado

Orthothrips exilis Hood, 1954: 42 (Brasil: Belém, PA) (folhas mortas de assí Euterpe oleracea))

Hood, J.D. 1954. Brasilian Thysanoptera IV. Proceedings of the Biological Society of Washington, 67: 17-54.

Distribuição no Brasil: PA (Belém)

Habitat: folhas mortas de assai Euterpe oleracea

Orthothrips leptura Hood, 1952: 151 (Brasil: Belém, PA) (folhas mortas de Astrocaryum mumbaca)

Hood, J.D. 1952. Brasilian Thysanoptera II. Proceedings of the Biological Society of Washington, 65: 141-174.

Distribuição no Brasil: PA (Belém)

Habitat: folhas mortas de Astrocaryum mumbaca

Orthothrips stilifer (Hood, 1957: 170) (Apoxythrips) (Brasil: Nova Teutônia, SC) (não dado)

Hood, J.D. 1957. New Brazilian Thysanoptera. Proceedings of the Biological Society of Washington, 70: 129-180.

Distribuição no Brasil: SC (Nova Teutônia)

Habitat: folhas caidas

\section{Gênero Pedoeothrips}

Pedoeothrips nigritus Hood, 1954: 43 (Brasil: Nova Teutônia, SC) (não dado)

Hood, J.D. 1954. Brasilian Thysanoptera IV. Proceedings of the Biological Society of Washington, 67: 17-54.

Distribuição no Brasil: SC (Nova Teutônia), SP (Juquiá)

Habitat: sem dado, Dracena marginata colorama

\section{Gênero Plagiothrips}

Plagiothrips eugeniae (Costa Lima, 1935: 28) (Gynaikothrips) (Brasil: RJ) (galhas em folhas de Eugenia sp.) Costa Lima, A.M. da. 1935. Tisanopterocecidias do Brasil. O Campo, 6(7): : 25-29.

Sinônimo: Gynaikothrips priesneri Costa Lima, 1937 
Costa Lima, A.M. da. 1937. Insetos do Brasil. XX. Thysanoptera. O Campo, 8(89): 17-24.

Distribuicão no Brasil: RJ

Habitat: galhas em folhas de Eugenia sp.

\section{Gênero Plectrothrips}

Plectrothrips antennatus Hood, 1908: 370

Hood, J.D. 1908. New genera and species of Illinois Thysanoptera. Bulletin Illinois State Laboratory of Natural History, 8: 361-379.

Distribuicão no Brasil: SP (Ribeirão Preto)

Habitat:

Plectrothrips bicuspis Hood, 1957: 174 (Brasil: Rondon, PR) (ramos mortos)

Hood, J.D. 1957. New Brazilian Thysanoptera. Proceedings of the Biological Society of Washington, 70: 129-180.

Distribuicão no Brasil: PR (Rondon)

Habitat: ramos mortos

Plectrothrips glaber Hood, 1954: 49 (Brasil: Nova Teutônia, SC) (ramos mortos)

Hood, J.D. 1954. Brasilian Thysanoptera IV. Proceedings of the Biological Society of Washington, 67: 17-54.

Distribuição no Brasil: SC, RJ

Habitat: ramos mortos

Plectrothrips hoodi Okajima, 1981: 322 (Brasil)

Okajima, S. 1981. A revision of the tribe Plectrothripini of fungus-geeding Thysanoptera (Phlaeothripidae:

Phlaeothripinae). Systematic Entomology, 6: 291-336.

Distribuiç̃̃o no Brasil: PR, MS

Habitat:

Plectrothrips nigricornis Okajima, 1981: 326 (Brasil: PR)

Okajima, S. 1981. A revision of the tribe Plectrothripini of fungus-geeding Thysanoptera (Phlaeothripidae:

Phlaeothripinae). Systematic Entomology, 6: 291-336.

Distribuicãa no Brasil:

Habitat:

Plectrothrips parvus Priesner, 1921: 203 (Paraguai unter Rinde) (desconhecido)

Priesner, H. 1921. Neue und Wenig bekannte Thysanopteren der neotropischen Fauna aus der Sammlung des Berliner Zoologischen Museums. Deutsche Entomologische Zeitschrift (Berliner entomologische Zeitschrift und Deutsche Entomologische Zeitschrift in Wiedervereinigung) 3: 187-223.

Distribuicão no Brasil: PR (Rondon)

Habitat: ramo morto

Plectrothrips rotundus Okajima, 1981: 329 (Brasil: PR)

Okajima, S. 1981. A revision of the tribe Plectrothripini of fungus-geeding Thysanoptera (Phlaeothripidae: Phlaeothripinae). Systematic Entomology, 6: 291-336.

Distribuicão no Brasil: PR

Habitat:

Plectrothrips thoracicus Hood, 1954: 48 (Brasil: Belém, PA) (Hevea sp.)

Hood, J.D. 1954. Brasilian Thysanoptera IV. Proceedings of the Biological Society of Washington, 67: 17-54.

Distribuicão no Brasil: PA (Belém)

Habitat: Hevea sp., Bixa sp.

Pleurothrips collaris (Hood, 1925: 58) (Eurythrips) (Trinidade) (árvore morta na floresta) Hood, J.D. 1925. New neotropical thysanoptera collected by C.B. Williams. Psyche, 32(1): 48-69.

Distribuição no Brasil: PA 
Habitat:

\section{Gênero Podothrips}

Podothrips bambusae Hood, 1949: 33 (Brasil: Jacarepaguá, RJ) (bambu)

Hood, J.D. 1949. Brasilian Thysanoptera I. Revista de Entomologia, 20(1-3): 3-88.

Distribuição no Brasil: RJ (Jacarepaguá/Rio de Janeiro), SP (Piracicaba e outros)

Habitat: bambu, ramos mortos

Podothrips brasiliensis Hood, 1949: 36 (Brasil: Serra da Cantareira, Franco da Rocha, SP) (gramínea)

Hood, J.D. 1949. Brasilian Thysanoptera I. Revista de Entomologia, 20(1-3): 3-88.

Distribuição no Brasil: SP (Serra da Cantareira/Franco da Rocha)

Habitat: gramínea

Podothrips paraensis Hood, 1952: 147 (Brasil: Belém, PA) (ramos mortos de Hevea sp.)

Hood, J.D. 1952. Brasilian Thysanoptera III. Proceedings of the Biological Society of Washington, 65: 141-174.

Distribuicão no Brasil: PA (Belém)

Habitat: ramos mortos de Hevea sp.

\section{Gênero Preeriella}

Preeriella discors Hood, 1957: 179 (Brasil: Nova Teutônia, SC) (Trichilia sp.)

Hood, J.D. 1957. New Brazilian Thysanoptera. Proceedings of the Biological Society of Washington, 70: 129-180.

Distribuição no Brasil: SC (Nova Teutônia)

Habitat: Trichilia sp.

Preeriella fumosa Hood, 1957: 178 (Brasil: Nova Teutônia, SC) (Solanum sp.)

Hood, J.D. 1957. New Brazilian Thysanoptera. Proceedings of the Biological Society of Washington, 70: 129-180.

Distribuição no Brasil: SC (Nova Teutônia)

Habitat: Solanum sp., em capoeira, sem dados

Preeriella macilenta Hood, 1957: 177 (Brasil: Belém, PA) (trepadeirass mortas de árvore Papaya sp.)

Hood, J.D. 1957. New Brazilian Thysanoptera. Proceedings of the Biological Society of Washington, 70: 129-180.

Distribuição no Brasil: PA (Belém)

Habitat: ramos mortos de Papaya sp.

Preeriella marginata Hood, 1957: 178 (Brasil: Nova Teutônia, SC) (Ahchornea sp. ou Trichilia sp.)

Hood, J.D. 1957. New Brazilian Thysanoptera. Proceedings of the Biological Society of Washington, 70: 129-180.

Distribuicão no Brasil: SC (Nova Teutônia)

Habitat: Ahchornea sp. ou Trichilia sp., Cedrella sp.

\section{Gênero Priesnerothrips}

Priesnerothrips amazonicus (Hood, 1954: 50) (Myopothrips) (Brasil: Belém, PA) (ramos mortos) Hood, J.D. 1954. Brasilian Thysanoptera IV. Proceedings of the Biological Society of Washington, 67: 17-54. Distribuicão no Brasil: PA (Belém), MS

Habitat: ramos mortos

\section{Gênero Pristothrips}

Pristothrips aaptus Hood, 1925: 62 (Trinidade) (cacau)

Hood, J.D. 1925. New Tropical Thysanoptera collected by C.B. Williams. Psyche, 32(1): 48-69

Distribuicão no Brasil: SC, SP (Piracicaba e outros?) 
Habitat: ramos vivos de citros com líquen

\section{Gênero Psalidothrips}

Psalidothrips conciliatus Hood, 1956(1955): 110 (Brasil: Belém, PA) (ramos mortos de Heliconia psittacorum)

Hood, J.D. 1956(1955). Brasilian Thysanoptera VI. Revista Brasileira de Entomologia, 4: 51-160.

Distribuição no Brasil: PA (Belém)

Habitat: ramos mortos de Heliconia pasittacorum, Ravenala guianensis e Astrocaryum mumbaca

Psalidothrips dissidens Hood, 1956(1955): 120 (Brasil: Belém, PA) (folhas mortas socratea Iriartea exorrhiza) Hood, J.D. 1956(1955). Brasilian Thysanoptera VI. Revista Brasileira de Entomologia, 4: 51-160.

Distribuicão no Brasil: PA (Belém)

Habitat: folhas mortas de socratea (Iriartea exorrhiza)

Psalidothrips longistylus Okajima, 1983: 8 (Trinidade)

Okajima, S. 1983. Studies on some Psalidothrips species with key to the world species (Thysanoptera:

Phlaeothripidae). Joumal of Natural History, 17: 1-13.

Distribuição no Brasil: AM

Habitat:

Psalidothrips retifer Hood, 1956(1955): 117 (Brasil: Belém, PA) (folhas mortas de Euterpe oleracea)

Hood, J.D. 1956(1955). Brasilian Thysanoptera VI. Revista Brasileira de Entomologia, 4: 51-160.

Distribuição no Brasil: PA (Belém)

Habitat: folhas mortas de Euterpe oleracea

Psalidothrips umbraticus Hood, 1956(1955): 114 (Brasil: Belém, PA) (folhas mortas de Astrocaryum mumbaca em uma densa capoeira)

Hood, J.D. 1956(1955). Brasilian Thysanoptera VI. Revista Brasileira de Entomologia, 4: 51-160.

Distribuicão no Brasil: PA (Belém)

Habitat: folhas mortas de Astrocaryum mumbaca

\section{Gênero Pseudophilothrips}

Pseudophilothrips ichini (Hood, 1949: 42) (Liothrips) (Brasil: Jacarepaguá, Rio de Janeiro, RJ) (folhas jovens de Ichinus terebenthifolius)

Hood, J.D. 1949. Brasilian Thysanoptera I. Revista de Entomologia, 20(1-3): 3-88.

Distribuição no Brasil: RJ (Jacarepaguá/Rio de Janeiro; Itha da Gipóia, próximo à Angra dos Reis), PR??? (Paraquara)

Habitat: folhas jovens de Ichinus terebintifolius

\section{Gênero Schasothrips}

Schasothrips anadenus Hood, 1957: 133 (Brasil: Nova Teutônia, SC) (folhas caídas)

Hood, J.D. 1957. New Brazilian Thysanoptera. Proceedings of the Biological Society of Washington, 70: 129-180.

Distribuicão no Brasil: SC (Nova Teutônia), RS (Erechim)

Habitat: sob folhas caídas

\section{Gênero Sedulothrips}

Sedulothrips tristis Hood, 1934: 434 (Panamá: Barro Colorado Island, Zona do Canal) (ramos mortos de várias árvores)

Hood, J.D. 1934. New Thysanoptera from Panama. Joumal of the New York Entomological Society, 41: 407-434.

Distribuicão no Brasil: PA (parte superior do Rio Paru do Oeste), SP (Piracicaba) 
Habitat: rachaduraa em um tronco de árvore; galhos mortos

Sedulothrips vigilans (Hood, 1913: 123) (Polyommatothrips) (Panamá)

Hood, J.D. 1913. On a collection of Thysanoptera from Panama. Psyche, 20(4): 119-124.

Sinônimo: Sedulothrips brevispinosus Moulton, 1933: 401 (Brasil) (sem dados) (coletado por O Conde, ?ES)

Moulton, D. 1933. The Thysanoptera of South America IV. Revista de Entomologia, 3(3): 385-419.

Distribuicão no Brasil: SP (Piracicaba), ? ES

Habitat: ramo morto com líquen

\section{Sedulothrips sp.}

Distribuicão no Brasil: SP (Piracicaba)

Habitat: ramo morto (protegido e recebendo bastante sol)

\section{Gênero Smicrothrips}

Smicrothrips particula Hood, 1952: 173 (Brasil: Nova Teutônia, SC) (Lantana sp.)

Hood, J.D. 1952. Brasilian Thysanoptera III. Proceedings of the Biological Society of Washington, 65: 141-174.

Distribuicão no Brasil: SC (Nova Teutônia)

Habitat: Lantana sp.

\section{Gênero Sophiothrips}

Sophiothrips comptus Hood, 1956(1955): 69 (Brasil: Nova Teutônia, SC) (sem dado)

Hood, J.D. 1956(1955). Brasilian Thysanoptera VI. Revista Brasileira de Entomologia, 4: 51-160.

Distribuição no Brasil: SC (Nova Teutônia)

Habitat: sem dado

Sophiothrips politus Hood, 1956(1955): 72 (Brasil: Belém, PA) (ramos mortos de Hevea sp.)

Hood, J.D. 1956(1955). Brasilian Thysanoptera VI. Revista Brasileira de Entomologia, 4: 51-160.

Distribuicão no Brasil: PA (Belém)

Habitat: ramos mortos de Hevea sp.

Sophiothrips scamosus Hood, 1933: 426 (Panamá: Porto Bello) (sob a casca de ramos mortos)

Hood, J.D. 1933. Joumal of the New York Entomological Society, 41(4): 407-434

Distribuição no Brasil: RJ (Jacarepaguá/Rio de Janeiro)

Habitat: ramos mortos

Sophiothrips verrucosus Hood, 1956(1955): 75 (Brasil: Jacarepaguá, Rio de Janeiro, RJ) (ramos mortos) Hood, J.D. 1956(1955). Brasilian Thysanoptera VI. Revista Brasileira de Entomologia, 4: 51-160.

Distribuicão no Brasil: RJ (Jacarepaguá/Rio de Janeiro)

Habitat: ramos mortos

\section{Gênero Strepterothrips}

Strepterothrips brasilianus (Hood, 1952: 155) (Arcyothrips) (Brasil: Nova Teutônia, SC) (ramos mortos) Hood, J.D. 1952. Brasilian Thysanoptera III. Proceedings of the Biological Society of Washington, 65: 141-174. Distribuicão no Brasil: SC (Nova Teutônia), SP (São Carlos)

Habitat: ramos mortos

Strepterothrips conradi Hood, 1933: 431 (Panamá: Barro Colorado Island, Zona do Canal) (ramos mortos de várias espécies de árvores e vines)

Distribuição no Brasil: PA

Habitat: 


\section{Gênero Symphyothrips}

Symphyothrips caliginosus Hood, 1952: 163 (Brasil: Nova Teutônia, SC) (ramos secos)

Hood, J.D. 1952. Brasilian Thysanoptera III. Proceedings of the Biological Society of Washington, 65: 141-174.

Distribuicão no Brasil: SC

Habitat: ramos secos

\section{Gênero Terthrothrips}

Terthrothrips balteatus Hood, 1957: 152 (Brasil: Nova Teutônia, SC) (sob folhas caídas)

Hood, J.D. 1957. New Brazilian Thysanoptera. Proceedings of the Biological Society of Washington, 70: 129-180.

Distribuicão no Brasil: SC (Nova Teutônia)

Habitat: sob folhas caídas

Terthrothrips bicinctus Hood, 1954: 32 (Brasil: Nova Teutônia, SC) (não dado)

Hood, J.D. 1954. Brasilian Thysanoptera IV. Proceedings of the Biological Society of Washington, 67: 17-54.

Distribuicãa no Brasil: SC (Nova Teutônia)

Habitat: não dado

Terthrothrips brunneus Hood, 1957: 148 (Brasil: Nova Teutônia, SC) (sob folhas caídas)

Hood, J.D. 1957. New Brazilian Thysanoptera. Proceedings of the Biological Society of Washington, 70: 129-180. Distribuicão no Brasil: SC (Nova Teutônia)

Habitat: sob folhas caídas

Terthrothrips bucculentus Hood, 1957: 145 (Brasil: Nova Teutônia, SC) (sob folhas caídas)

Hood, J.D. 1957. New Brazilian Thysanoptera. Proceedings of the Biological Society of Washington, 70: 129-180.

Distribuição no Brasil: SC (Nova Teutônia)

Habitat: sob folhas caídas

Terthrothrips bullifer Hood, 1957: 149 (Brasil: Nova Teutônia, SC) (sob folhas caídas)

Hood, J.D. 1957. New Brazilian Thysanoptera. Proceedings of the Biological Society of Washington, 70: 129-180.

Distribuição no Brasil: SC (Nova Teutônia)

Habitat: sob folhas caídas

Terthrothrips carens Hood, 1957: 147 (Brasil: Nova Teutônia, SC) (sob folhas caídas)

Hood, J.D. 1957. New Brazilian Thysanoptera. Proceedings of the Biological Society of Washington, 70: 129-180.

Distribuição no Brasil: SC (Nova Teutônia), RS (Erechim)

Habitat: sob folhas caídas

Terthrothrips fuscatus Hood, 1954: 29 (Brasil: Nova Teutônia, SC) (não mencionado)

Hood, J.D. 1954. Brasilian Thysanoptera IV. Proceedings of the Biological Society of Washington, 67: 17-54.

Distribuicão no Brasil: SC (Nova Teutônia)

Habitat: não mencionado

Terthrothrips hebes Hood, 1957: 152 (Brasil: Nova Teutônia, SC) (sob folhas caidas)

Hood, J.D. 1957. New Brazilian Thysanoptera. Proceedings of the Biological Society of Washington, 70: 129-180.

Distribuicão no Brasil: SC (Nova Teutônia)

Habitat: sob folhas caídas

Terthrothrips impolitus Hood, 1957: 153 (Brasil: Nova Teutônia, SC) (sob folhas caídas)

Hood, J.D. 1957. New Brazilian Thysanoptera. Proceedings of the Biological Society of Washington, 70: 129-180.

Distribuicão no Brasil: SC (Nova Teutônia)

Habitat: sob folhas caídas 
Terthrothrips irretitus Hood, 1957: 150 (Brasil: Nova Teutônia, SC) (sob folhas caidas)

Hood, J.D. 1957. New Brazilian Thysanoptera. Proceedings of the Biological Society of Washington, 70: 129-180.

Distribuicão no Brasil: SC (Nova Teutônia)

Habitat: sob folhas caídas

Terthrothrips luteolus Hood, 1957: 151 (Brasil: Nova Teutônia, SC) (sob folhas caídas)

Hood, J.D. 1957. New Brazilian Thysanoptera. Proceedings of the Biological Society of Washington, 70: 129-180.

Distribuição no Brasil: SC (Nova Teutônia)

Habitat: sob folhas caídas)

Terthrothrips marginatus Gerdes, 1984: 405 (Brasil)

Gerdes, C.F. 1984 ou 1980 ? Eurythrips and Terthrothrips (Thysanoptera: Phlaeothripidae) from southern Brazil, with one new species, new collection sites, and key. Proceedings of the Entomological Society os Washington, 86: 400-410.

Distribuicão no Brasil: SC (Seara)

Habitat: sem dado

Terthrothrips peltatus Hood, 1957: 146 (Brasil: Nova Teutônia, SC) (sob folhas caídas)

Hood, J.D. 1957. New Brazilian Thysanoptera. Proceedings of the Biological Society of Washington, 70: 129-180.

Distribuicão no Brasil: SC (Nova Teutônia)

Habitat: sob folhas caídas

Terthrothrips percultus Hood, 1957: 154 (Brasil: Nova Teutônia, SC) (sob folhas caidas)

Hood, J.D. 1957. New Brazilian Thysanoptera. Proceedings of the Biological Society of Washington, 70: 129-180.

Distribuição no Brasil: SC (Nova Teutônia)

Habitat: sob folhas caídas

Terthrothrips sanguinolentus Bergroth, 1896: 66 (Phloeothrips) (Brasil: Blumenau, SC) (desconhecido)

Bergroth, E. 1896. Annales de la Société Entomologiqué de Belgique, 40(2): 66-67.

Distribuicão no Brasil: SC (Blumenau)

Habitat: sem dado

Terthrothrips serratus Hood, 1954: 31 (Brasil: Nova Teutônia, SC) (não dado)

Hood, J.D. 1954. Brasilian Thysanoptera IV. Proceedings of the Biological Society of Washington, 67: 17-54.

Distribuicão no Brasil: SC (Nova Teutônia)

Habitat: não dado

Terthrothrips unicinctus Hood, 1954: 30 (Brasil: Nova Teutônia, SC) (não dado)

Hood, J.D. 1954. Brasilian Thysanoptera IV. Proceedings of the Biological Society of Washington, 67: 17-54.

Distribuicão no Brasil: SC (Nova Teutônia)

Habitat: não dado

Terthrothrips viduus Hood, 1957: 147 (Brasil: Nova Teutônia, SC) (sob folhas caidas)

Hood, J.D. 1957. New Brazilian Thysanoptera. Proceedings of the Biological Society of Washington, 70: 129-180.

Distribuicão no Brasil: SC (Nova Teutônia)

Habitat: sob folhas caídas

Terthrothrips sp.

Distribuição no Brasil: SP (Piracicaba)

Habitat: coletado com arnadilha de sucção

\section{Gênero Trachythrips}

Trachythrips epimeralis Hood, 1941: 195 (Brasil: Anápolis, GO) (genipapo Genipapa americana, Rubiaceae) Hood, J.D. 1941. A century of new American Thysanoptera II. Revista de Entomologia, 12(1-2): 139-243. 
Distribuicão no Brasil: GO (Anápolis), RS, SP (Piracicaba)

Habitat: genipapo (Genipapa americana), folhas de Eucalyptus sp. mortas no chão

\section{Gênero Trichinothrips}

Trichinothrips callypechys Hood, 1952: 147 (Brasil: Santarém, PA) (ramos mortos)

Hood, J.D. 1952. Brasilian Thysanoptera III. Proceedings of the Biological Society of Washington, 65: 141-174.

Distribuição no Brasil: PA (Santarém), RJ (Jacarepaguá/Rio de Janeiro)

Habitat: ramos mortos, vegetação baixa misturada

Trichinothrips latifrons Hood, 1956(1955): 83 (Brasil: São Carloṡ, SP) (ramos mortos e morrendo de laranjeira)

Hood, J.D. 1956(1955). Brasilian Thysanoptera VI. Revista Brasileira de Entomologia, 4: 51-160.

Distribuição no Brasil: SP (São Carlos)

Habitat: ramos mortos e morrendo de laranjeira

Trichinothrips panamensis Hood, 1935: 91 (Panamá)

Hood, J.D. 1935. Ten new Thysanoptera from Panama. Proceedings of the Biological Society of Washington, 48: 86106.

Distribuicão no Brasil: SP

Habitat:

Trichinothrips sensilis Hood, 1952: 148 (Brasil: Jacarepaguá, Rio de Janeiro, RJ) (ramos mortos)

Hood, J.D. 1952. Brasilian Thysanoptera III. Proceedings of the Biological Society of Washington, 65: 141-174.

Distribuição no Brasil: PA (Belém), RJ (Jacarepaguá)

Habitat: ramos mortos

\section{Gênero Tropothrips}

Tropothrips borgmeieri Hood, 1949: 71 (Brasil: Petrópolis, RJ) (ramos mortos em locais sombreados)

Hood, J.D. 1949. Brasilian Thysanoptera I. Revista de Entomologia, 20(1-3): 3-88.

Distribuição no Brasil: RJ (Petrópolis, Jacarepaguá/Rio de Janeiro)

Habitat: ramos mortos em locais sombreados

\section{Gênero Trybomia}

Trybomia gossypii (Hood, 1915: 33)(Ommathrips) (Peru: Dept. de Piura, Catacaos) (algodão) Hood, J.D. 1915. Descriptions of new American Thysanoptera. Insecutor Inscitiae Menstruus, 3(1-4): 1-40.

Distribuicão no Brasil: SC (Nova Teutônia)

Habitat: sem dado

Trybomia intermedius (Bagnall, 1910: 384) (Liothrips) (Venezuela: Los Adjuntas) (flores)

Bagnall, R.S. 1910. A contribution towards a knowledge of the neotropical Thysanoptera. Journal of the Linnean Society, Zoology, 30(201): 369-387.

Sinônimo: Trybomia intermedia fiebrigi Priesner, 1921: 195 (Brasil: MG) (Tibouchina mutabilis)

Priesner, H. 1921. Neue und wenig bekannte Thysanopteren der neotropischen Fauna aus der Sammlung des Berliner Zoologischen Museums. Deutsche Entomologische Zeitung, 3: 187-223.

Distribuição no Brasil: MG, RJ, SP

Habitat: Cyperus surinamensis e outros hospedeiros; Tibouchina mutabilis; flores de crisântemo

Trybomia mendesi Moulton, 1933: 259 (Brasil: Jardo, Botânico da Estação Biológica, Monte Itatiaya, RJ) (sem dado)

Moulton, D. 1933. Three new Urothripidae from Panama. Proceedings of the Biological Society of Washington, 46: 213-216.

Distribuiç̃o no Brasil: RJ (Monte Itatiaya) 
Habitat: sem dado

Trybomia sp.

Distribuicão no Brasil: SP (Mata de Santa Genebra/Campinas, Piracicaba)

Habitat: Datura sp., folhas de árvore

\section{Gênero Trypanothrips}

Trypanothrips coxalis Hood, 1957: 173 (Brasil: Nova Teutônia, SC) (ramos mortos)

Hood, J.D. 1957. New Brazilian Thysanoptera. Proceedings of the Biological Society of Washington, 70: 129-180. Distribuicão no Brasil: SC (Nova Teutônia)

Habitat: ramos mortos

\section{Gênero Tylothrips}

Tylothrips achaetus (Hood, 1957: 158) (Phragmothrips) (Brasil: Nova Teutônia, SC) (sob folhas caídas)

Hood, J.D. 1957. New Brazilian Thysanoptera. Proceedings of the Biological Society of Washington, 70: 129-180.

Distribuição no Brasil: SC (Nova Teutônia)

Habitat: sob folhas caídas

Tylothrips brasiliensis (Hood, 1957: 162) (Copiothrips) (Brasil: Nova Teutônia, SC) (sob folhas caídas) Hood, J.D. 1957. New Brazilian Thysanoptera. Proceedings of the Biological Society of Washington, 70: 129-180. Distribuição no Brasil: SC (Nova Teutônia)

Habitat: sob folhas caídas

Tylothrips caelatoris (Hood, 1954: 34) (Porcothrips) (Brasil: Nova Teutônia, SC) (não dado)

Hood, J.D. 1954. Brasilian Thysanoptera IV. Proceedings of the Biological Society of Washington, 67: 17-54.

Distribuicão no Brasil: SC (Nova Teutônia)

Habitat: não dado

Tylothrips cochlearius (Hood, 1954: 27) (Terthrothrips) (Brasil: Nova Teutônia, SC) (não dado)

Hood, J.D. 1954. Brasilian Thysanoptera IV. Proceedings of the Biological Society of Washington, 67: 17-54.

Distribuição no Brasil: SC (Nova Teutônia)

Habitat: não dado

Tylothrips consobrinus (Hood, 1954: 25) (Terthrothrips) (Brasil: Nova Teutônia, SC) (não dado)

Hood, J.D. 1954. Brasilian Thysanoptera IV. Proceedings of the Biological Society of Washington, 67: 17-54.

Distribuição no Brasil: SC (Nova Teutônia)

Habitat: não dado

Tylothrips crassus (Hood, 1954: 28) (Terthrothrips) (Brasil: Nova Teutônia, SC) (não dado)

Hood, J.D. 1954. Brasilian Thysanoptera IV. Proceedings of the Biological Society of Washington, 67: 17-54.

Distribuicão no Brasil: SC (Nova Teutônia)

Habitat: não dado

Tylothrips flaviventris (Hood, 1957: 160) (Copiothrips) (Brasil: Nova Teutônia, SC) (sob folhas caídas) Hood, J.D. 1957. New Brazilian Thysanoptera. Proceedings of the Biological Society of Washington, 70: 129-180. Distribuição no Brasil: SC (Nova Teutônia)

Habitat: sob folhas caídas

Tylothrips forticauda (Hood, 1954: 34) (Porcothrips) (Brasil: Nova Teutônia, SC) (não dado)

Hood, J.D. 1954. Brasilian Thysanoptera IV. Proceedings of the Biological Society of Washington, 67: 17-54.

Distribuicão no Brasil: SC (Nova Teutônia)

Habitat: não dado 
Tylothrips fulvescens (Hood, 1957: 161) (Copiothrips) (Brasil: Nova Teutônia, SC) (sob folhas caídas) Hood, J.D. 1957. New Brazilian Thysanoptera. Proceedings of the Biological Society of Washington, 70: 129-180. Distribuição no Brasil: SC (Nova Teutônia)

Habitat: sob folhas caídas

Tylothrips fuscifrons (Hood, 1957: 159) (Copiothrips) (Brasil: Nova Teutônia, SC) (sob folhas caídas) Hood, J.D. 1957. New Brazilian Thysanoptera. Proceedings of the Biological Society of Washington, 70: 129-180. Distribuição no Brasil: SC (Nova Teutônia)

Habitat: sob folhas caídas

Tylothrips gracilis (Hood, 1954: 29) (Terthrothrips) (Brasil: Nova Teutônia, SC) (sem dado)

Hood, J.D. 1954. Brasilian Thysanoptera IV. Proceedings of the Biological Society of Washington, 67: 17-54.

Distribuicão no Brasil: SC (Nova Teutônia)

Habitat: sem dado

Tylothrips inuncatus (Hood, 1957: 157) (Phragmothrips) (Brasil: Nova Teutônia, SC) (sob folhas caídas) Hood, J.D. 1957. New Brazilian Thysanoptera. Proceedings of the Biological Society of Washington, 70: 129-180. Distribuicão no Brasil: SC (Nova Teutônia)

Habitat: sob folhas caídas

Tylothrips longulus (Hood, 1954: 26) (Terthrothrips) (Brasil: Nova Teutônia, SC) (sem dado) Hood, J.D. 1954. Brasilian Thysanoptera IV. Proceedings of the Biological Society of Washington, 67: 17-54. Distribuição no Brasil: SC (Nova Teutônia)

Habitat: sem dado

Tylothrips majusculus (Hood, 1957: 155) (Phragmothrips) (Brasil: Nova Teutônia, SC) (sob folhas caidas) Hood, J.D. 1957. New Brazilian Thysanoptera. Proceedings of the Biological Society of Washington, 70: 129-180. Distribuicão no Brasil: SC (Nova Teutônia)

Habitat: sob folhas caídas

Tylothrips minor (Hood, 1954: 28) (Terthrothrips) (Brasil: Nova Teutônia, SC) (não dado)

Hood, J.D. 1954. Brasilian Thysanoptera IV. Proceedings of the Biological Society of Washington, 67: 17-54.

Distribuição no Brasil: SC (Nova Teutônia)

Habitat: não dado

Tylothrips paulus (Hood, 1957: 156) (Phragmothrips) (Brasil: Nova Teutônia, SC) (sob folhas caidas)

Hood, J.D. 1957. New Brazilian Thysanoptera. Proceedings of the Biological Society of Washington, 70: 129-180. Distribuição no Brasil: SC (Nova Teutônia), RS (Erechim)

Habitat: sob folhas caidas

Tylothrips striaticeps (Hood, 1954: 26) (Terthrothrips) (Brasil: Nova Teutônia, SC) (não dado) Hood, J.D. 1954. Brasilian Thysanoptera IV. Proceedings of the Biological Society of Washington, 67: 17-54. Distribuição no Brasil: SC (Nova Teutônia)

Habitat: não dado

Tylothrips subglaber (Hood, 1957: 161) (Copiothrips) (Brasil: Nova Teutônia, SC) (sob folhas caidas) Hood, J.D. 1957. New Brazilian Thysanoptera. Proceedings of the Biological Society of Washington, 70: 129-180. Distribuicão no Brasil: SC (Nova Teutônia)

Habitat: sob folhas caidas

Tylothrips ustulatus (Hood, 1957: 163) (Copiothrips) (Brasil: Nova Teutônia, SC) (sob folhas caídas) Hood, J.D. 1957. New Brazilian Thysanoptera. Proceedings of the Biological Society of Washington, 70: 129-180. Distribuicão no Brasil: SC (Nova Teutônia)

Habitat: sob folhas caídas 
Tylothrips sp.

Distribuicão no Brasil: SP (Juquiá)

Habitat: Dracena marginata colorama

\section{Gênero Xyphalothrips}

\section{Xyphalothrips sp.}

Distribuicão no Brasil:SP (Piracicaba)

Habitat: vagens abertas de Sesbania sp.

\section{Gênero Williamsiella}

Williamsiella bicoloripes Hood, 1925: 60 (Trinidade) (em feixes de madeira)

Hood, J.D. 1925. New Neotropical Thysanoptera collected by C.B. Williams. Psyche, 32(1): 48-69

Distribuição no Brasil: SC (Nova Teutônia)

Habitat: ramo seco

Williamsiella brasiliensis Priesner, 1937: 347 (Phthirothrips) (Brasil)

Priesner, H. 1937. Thysanopterologica V: Zwei neue Phlaeothripiden. Arbeiten uber morphologische und taxonomische Entomologie, 4: 347-350.

Distribuição no Brasil:

Habitat:

Williamsiella breviceps (Hood, 1925: 39) (Lissothrips) (Trinidade) (ramos mortos)

Hood, J.D. 1925. New Neotropical Thysanoptera collected by C.B. Williams. Psyche, 32(1): 48-69

Distribuição no Brasil:

Habitat:

Williamsiella brevisetis (Hood, 1950: 28) (Phthirothrips) (Brasil: Petrópolis, RJ) (ramos mortos e caídos de várias árvores no chão do monastério "Lar das Cegas")

Hood, J.D. 1950. Brasilian Thysanoptera II. Revista de Entomologia, 21(1-2): 1-113.

Distribuicão no Brasil: RJ (Petrópolis), SP (Boracéa/Salesópolis)

Habitat:

Williamsiella capitulatus (Hood, 1960: 63) (Lissothrips) (Brasil: Nova Teutônia, SC) (litter from primeval forest)

Hood, J.D. 1960. Six new Thysanoptera from Brazil. Revista Brasileira de Entomologia, 9: 57-68.

Distribuição no Brasil: SC (Nova Teutônia)

Habitat: serrapilheira de floresta pninána

Williamsiella longiceps (Hood, 1960: 59) (Phthirothrips) (Brasil)

Hood, J.D. 1960. Six new Thysanoptera from Brazil. Revista Brasileira de Entomologia, 9: 57-68.

Distribuição no Brasil: SC (Nova Teutônia)

Habitat: serrapilheira de floressta primána

Williamsiella nemoralis (Hood, 1954: 25) (Phthirothrips) (Brasil: Belém, PA) (folha morta de Ravenala guianensis em uma densa capoeira)

Hood, J.D. 1954. Brasilian Thysanoptera IV. Proceedings of the Biological Society of Washington, 67: 17-54.

Distribuição no Brasil: PA (Belém)

Habitat: folha morta de Ravenala guianensis

Williamsiella tricosus (Hood, 1960: 67) (Lissothrips) (Brasil: Nova Teutônia, SC) (serrapilheira de floresta primána)

Hood, J.D. 1960. Six new Thysanoptera from Brazil. Revista Brasileira de Entomologia, 9: 57-68.

Distribuição no Brasil: SC (Nova Teutônia) 
Habitat: serrapilheira de floresta primánias

Williamsiella ventralis (Hood, 1954: 24) (Lissothrips) (Brasil: Nova Teutônia, SC) (ramos mortos)

Hood, J.D. 1954. Brasilian Thysanoptera IV. Proceedings of the Biological Society of Washington, 67: 17-54. Distribuicão no Brasil: SC (Nova Teutônia)

Habitat: ramos mortos

\section{Gênero Zaliothrips}

Zaliothrips imitator Hood, 1956(1955): 78 (Brasil: São Carlos, SP) (ramos mortos)

Hood, J.D. 1956(1955). Brasilian Thysanoptera VI. Revista Brasileira de Entomologia, 4: 51-160.

Distribuicão no Brasil: SP (São Carlos)

Habitat: ramos mortos

Zaliothrips longisetosus Hood, 1956(1955): 79 (Brasil: Boracéa, Salesópolis, SP) (ramos mortos) Hood, J.D. 1956(1955). Brasilian Thysanoptera VI. Revista Brasileira de Entomologia, 4: 51-160.

Distribuição no Brasil: SP (Boracéa/Salesópolis)

Habitat: ramos mortos

Zaliothrips luteolus (Hood, 1956(1955): 80) (Brasil: Belém, PA) (ramos mortos de Hevea sp.) Hood, J.D. 1956(1955). Brasilian Thysanoptera VI. Revista Brasileira de Entomologia, 4: 51-160. Distribuicão no Brasil: PA (Belém)

Habitat: ramos mortos de Hevea sp., Andropogon sp. e outras vegetações vivas e mortas 


\section{O GÊNERO Frankliniella NO BRASIL}

\section{RESUMO}

Das 298 espécies descritas do gênero Frankliniella, apenas 160 são válidas. No Brasil, um total de 40 espécies foi constatado, sendo 18 descritas com base em material nativo. Quatorze espécies não foram incluídas no estudo por vários motivos. Uma chave para identificação de 27 espécies de Frankliniella é apresentada, incluindo uma espécie nova, aqui descrita, assim como a caracterização morfológica e a ilustração de cada espécie.

\section{SUMMARY}

The genus Frankliniella has 298 described species, however 160 valid names. In Brazil, 40 species were collected, 18 based in native specimens. Fourteen species were not included in this study for different reasons. A key to identify 27 Frankliniella species is presented, including a new species, here described, and the morphological characterization and illustrations of each one.

\subsection{Introdução}

Frankliniella Kamy, 1910 é o segundo maior gênero da familia Thripidae, com cerca de 300 (298) espécies descritas, sendo 160 o número de espécies válidas (Nakahara, 1997; Nakahara \& Monteiro, 1999). A maioria das espécies (152) foi descrita da América do Sul por J.D. Hood e D. Moulton (Mound \& Marullo, 1996). Um total de 40 espécies é registrado no Brasil.

Os adultos de Frankliniella caracterizam-se por apresentar: antena geralmente com 8 segmentos ( 7 segmentos em 3 espécies, não constatadas no Brasil); segmentos antenais III e IV 
com sensilos em forma de furca; cabeça com cerdas ocelares I presentes e III, variáveis em posição (posição 1: anterior ao triângulo ocelar; 2: entre as duas tangentes paralelas dos ocelos anteriores e postesriores; 3: dentro do triângulo ocelar; 4: entre as tangentes dos ocelos posteriores); olhos compostos com geralmente 4 omatídeos coloridos (5 em F. schultzei, ausente em poucas espécies); pronoto geralmente com dois pares cerdas longas na margem anterior (ântero-marginal e ântero-angular, embora algumas vezes sejam reduzidos) e dois pares de cerdas póstero-angulares longas; margem posterior do pronoto com o par de cerdas medianas (pm I) menores que o par de cerdas submedianas (pm II); cerdas medianas do mesonoto com inserção próxima à margem anterior do esclerito; asas geralmente completamente desenvolvidas em ambos os sexos, ambas nervuras com uma fileira completa de cerdas, escama geralmente com 5 cerdas venais e 1 cerda discal; tergito II com três cerdas látero-marginais e uma cerda na extremidade doo ângulo anterior do pleurotergito; tergitos V-VIII com um ctenídeo em cada lateral, no VIII em posição ântero-lateral em relação ao espiráculo; esternito I com três cerdas ântero-medianas pequenas; esternitos III-VII sem cerdas discais; três pares de cerdas marginais no esternito VII, com inserção na margem ou próxima a ela (Mound \& Nakahara, 1993; Mound \& Marullo, 1996).

A chave para as espécies apresentada por Moulton (1933) foi baseada principalmente na coloração, assim como sua revisão sobre as espécies do gênero (Moulton, 1948), com chaves de identificação para espécies, agnupando-as em subgêneros. Muitas das espécies descritas por Moulton foram colocadas em sinonimias por outros pesquisadores. Mound \& Marullo (1996) apresentaram uma chave para 80 espécies da região do Caribe e América Central e Nakahara (1997) listou as espécies do mundo. Sakimura \& O'Neill (1979) revisaram o grupo minuta e descreveram novas espécies, redefinindo o gênero de forma mais ampla e compreensiva. Mound \& Nakahara (1993) reuniram os caracteres morfológicos que podem ser utilizados para definir o gênero e revisaram muitos dos problemas de reconhecimento de espécies devido à variações na cor e estrutura, o que não havia sido considerado adequadamente por outros pesquisadores.

Embora o gênero seja muito bem caracterizado e apresente grande diversidade, as espécies de Frankliniella são freqüentemente difíceis de serem distingüidas, por serem muito similares. Algumas espécies podem ser caracterizadas por um ou vários caracteres, mas outras espécies, como $F$. occidentalis, não apresentam caracteres que as definam. Algumas espécies variam em cor e estruturas anatômicas em função do sexo (machos geralmente mais claros que as fêmeas), maturação do adulto (adultos recém-emergidos são mais claros), região geográfica (temperatura, altitude, entre outros), fonte alimentar (hospedeiro), armazenagem (meio 
preservante, tempo e temperatura) e preparo de lâminas de microscopia. Por essas razões, e também pela ausência de estudos e grande número de espécies descritas, muitas das espécies foram sinonimizadas e os táxons reconfirmados.

\subsection{Material e Métodos}

As observações morfológicas foram realizadas em espécimens depositados nas coleções da ESALQ/USP (Entomologia) e do USNM e em outros espécimens, preparados e montados em lâminas de microscopia, segundo o método descrito em Mound \& Ptikin, (1972) e Mound \& Manullo (1996). Nesse caso, o preparo e a montagem foram feitos sob microscópio estereoscópico e as observações morfológicas, sob microscópio biológico. $\mathbf{O}$ meio de montagem foi o Hoyer ou o bálsamo-do-canadá.

Os caracteres morfológicos dos adultos das espécies de Frankliniella foram ilustrados com auxílio de uma câmara clara acoplada a um microscópio biológico. A terminologia utilizada foi baseada em Palmer et al. (1989) e Mound \& Marullo (1996). As escalas nas figuras medem $0,1 \mathrm{~mm}$. A terminologia utilizada encontra-se no Anexo 2.

A chave apresentada inclui 27 espécies, sendo uma nova. A descrição da nova espécie será incluída neste trabalho, porém, ainda não será atribuído nome espécífico, pois de acordo com o Capítulo III, Artigo 9, item 11 do ICNZ (International Code of Zoological Nomenclature) (1985), a tese não é considerada publicação apropriada para a atribuição de novos nomes. Algumas dessas espécies provavelmente serão reconhecidas como sinonímias quando estudos de campo adicionais forem conduzidos. Esta chave não inclui a forma marrom escura, quase negra, de $F$. occidentalis, que não foi registrada no Brasil. Mas, é conveniente mencionar que essa forma é comumente encontrada em grandes altitudes. Também, com relação à $F$. schultzei, há duas formas, variando em coloração: a marrom e a amarela. A forma marrom de $F$. schultzei é a comumente encontrada no País, mas a forma amarela, também conhecida como "sulfurea", ainda não foi constatada. No entanto, independentemente da coloração, ambas as formas, tanto de $F$. occidentalis como de $F$. schultzei, apresentam as mesmas características morfológicas e necessitam de estudos posteriores para determinar os padrões de variação cromática.

Assim, as espécies de Frankliniella incluídas no estudo foram: F. annulipes Hood, 1915, F. bertelsi (De Santis, 1967), F. bondari Hood, 1941, F. brevicaulis Hood, 1937, F. brunnea Priesner, 1932, F. condei John, 1927, F. desantisi Sakimura \& O'Neill, 1979, F. distinguenda 
Bagnall, 1919, F. fulvipennis Moulton, 1933, F. fulvipes Bagnall, 1919, F. gardeniae Moulton, 1948, F. gemina Bagnall, 1919, F. hemerocallis Crawford, 1948, F. insularis (Franklin, 1908), F. longipennis ((Moulton, 1933), F. longispinosa Moulton, 1933, F. occidentalis (Pergande, 1895), $F$. oxyura Bagnall, 1919, $F$. peruviana Hood, 1937, F. rodeos, Moulton, 1933, $F$. schultzei (Trybom, 1910), F. simplex Priesner, 1924, F. trinidadensis Hood, 1941, F. varipes Moulton, 1933, F. williamsi Hood, 1915, F. zucchini Nakahara \& Monteiro, 1999 e Frankliniella sp. $n$.

As ilustrações da antena, cabeça, pronoto, metanoto, tergito abdominal VIII e tergito abdominal IX das 27 espécies de Frankliniella relacionadas na chave encontram-se nas pranchas I-XIV.

Os espécimens estudados encontram-se depositados no Museu de Entomologia, ESALQ/USP, em Piracicaba, SP (Apêndice 1) e no United States Nacional History Museum (USNM), em Washington, D.C., EUA. (Apêndice 2).

\subsection{Resultados e Discussão}

A nova espécie de Frankliniella faz parte da coleção do Dr. J.D. Hood, depositada no USNM, Washington, D.C., onde foi reconhecida como tal pelo Dr. Laurence A. Mound.

\subsubsection{Frankliniella sp. n. (Figura 27 das pranchas II, IV, VIII, IX, XI e XIV)}

Fêmea: macróptera. Corpo de coloração marrom-dourado, cabeça e abdome um pouco mais escuros, asas anteriores marrons com o $1 / 4$ basal claro; pernas amarelas, exceto a margem externa sombreada de marrom dos fêmures anteriores; cerdas do corpo e das asas anteriores marromescuras. Segmento antenal I marrom; II quase totalmente marrom, distalmente menos escuro; III amarelo, com sombra marrom-clara distalmente; IV amarelo na metade proximal, com sombra marrom-clara distalmente; V amarelo no $1 / 4$ basal, com sombra marrom clara distalmente; VI a VIII com sombra marrom claro.

Cabeça: comprimento aproximadamente igual ao do pronoto e tão longa quanto larga; bochechas um tanto estreitas. Área interocelar lisa, sem esculturação, posteriormente as olhos compostos com estrias transversais amplamente espaçadas. Diâmetro do ocelo anterior $13 \mu$. Cerda ocelar I 
pequena, aproximadamente 7-9 $\mu$ de comprimento, longo à frente do ocelo anterior; cerdas ocelares II pequenas, laterais ao ocelo anterior e alinhadas com sua margem posterior, $12 \mu$; cerdas ocelares III bem desenvolvidas, na posição 2 , separadas por aproximadamente 1,5 vezes o diâmetro do ocelo anterior, 43-45 $\mu$ de comprimento. Cerda pós-coular (PO) i ausente; PO ii 7-9 $\mu$ de comprimento; PO iii 5-7 $\mu$ de comprimento, caudais aos ocelos posteriores e alinhadas com a margem posterior dos olhos compostos; PO iv maiores 21-28 $\mu$ long, caudais aos olhos compostos; PO v laterais e menores que iv, mas maiores que ii e iii, aproximadamente 12-14 $\mu$. Cone bucal arredondado, menor que a cabeça, extendendo até a metade do pró-esterno.

Antena: aproximadamente duas vezes maior que a cabeça; pedicelo do segmento III com uma leve angulação; segmentos III e IV com sensilos em formato de furca; segmento VIII distintamente mais longo que o VII.

Pronoto: retangular, mais largo (1/6) que longo, esculturação com estrias transversais irregularmente espaçadas e ocasionais anastomoses. Três pares de cerdas ântero-marginais (am) (um par maior entre dois pares menores); cerdas ântero-angulares (aa) aproximadamente 2.0 vezes maiores que as cerdas am; cerdas póstero-marginais e 1/6 do comprimento do pronoto (pm) II aproximadamente tão longas quanto as I; pm II, V e VI com aproximadamente o mesmo comprimento e menores que I; pm IV tão longa quanto II; cerdas póstero-angulares (pa) bem desenvolvidas, par interno menor que o externo, par externo aproximadamente 0.4 vezes o comprimento do pronoto.

Mesonoto: subtrapezoidal; área anterior com angulação e lisa, com 2 sensilos campaniformes; parte mediana e posterior esculturada, com algumas linhas oblíquas unidas; lateralmente com estrias longitudinais; 2 pares de cerdas próximas/sobre a margem postgerior (par interno menor que o externo).

Metanoto: terço anterior com esculturação mediana mais larga que longa; terços mediano e posterior com esculturação mais longa que larga; esculturação das laterais mais longas que largas; linhas da esculturação median fracas. Cerdas medianas inseridas na margem anterior, um pouco maiores que a $1 / 2$ do comprimento do metanoto; sensilos campaniformes ausentes. 
Asas anteriores: pouco convergente em direção ao ápice pontiagudo; cerdas da franja onduladas. Primeira nervura da asa anterior com 19-20 (19) cerdas; nervura posterior com 16-17(12-15) cerdas; escama com 5 cerdas marginais e 1 cerda discal.

Abdome: tergitos esculturados (poucas linhas transversais amplamente espaçadas) apenas lateralmente às cerdas medianas e sensilos campaniformes; cerdas medianas pequenas, no VII (12,70 $\mu$ de comprimento); margem posterior com craspedo de lobos curtos e truncados, lateralmente com formato triangular; par de ctenídeos (um em cada lateral) no IV (vestigial) ao VIII; VIII com pente póstero-marginal completo com aproximadamente 13 microtríquias, em formato triangular; IX com 2 pares de poros, cerdas $B_{1} 1 / 6$ do comprimento das $B_{2} ; X$ longitudinalmente dividido no $3 / 4$ distal. Margem posterior dos esternitos também com craspedo, como nos tergitos; II com 1 ou 2 cerdas discais; III com 2 áreas glandulares pequenas, circulares e claras (diâmetro transversal 5-7 $\mu$ ) no 1/3 anterior, em formato de poro.

Mediç̃es (fêmea holótipo, em microns): Comprimento do corpo 1761. Cabeça, comprimentol45; largura atrás dos olhos 160; cerda ocelar III 45; cerda pós-ocular iv 26. Pronoto, comprimento 152; largura 174; cerdas principais, menor am 10, am 24; aa 48, pm I 14, pm II 14, pa interna 57, pa externa 69. Asa anterior, comprimento 893. Tergito VIII - pente póstero-marginal, comprimento das cerdas medianas 14, diâmetro da base dos dentes 11 . Tergito $\mathrm{IX}$, comprimento 88, cerdas $B_{1} 119$, cerdas $B_{2} 143$; cerdas $B_{3} 155$. Tergito $X$, comprimento 76 , cerdas $B_{1} 133$. Antena - segmentos I-VIII, comprimento 24, 38, 55, 45, 40, 52, 178.

Holótipo: fêmea; Brasil: Nova Teutônia, Estado de Santa Catarina; hospedeiro desconhecido (nome da planta ilegivel); 27.v.1949; Fritz Plaumann col.; lâmina n 1874 da coleção do Dr. Hood; depositada no USNM, Washington, Maryland, EUA. Parátipo: fêmea; Brasil: Nova Teutônia, Estado de Santa Catarina; ramos mortos; 5.v.1949; Fritz Plaumann col.; lâmina ${ }^{\circ}$ 2018-S da coleção do Dr. Hood; depositada no USNM, Washington, Maryland, EUA.

Comentários: Essa espécie é descrita de apenas duas fêmeas coletadas no Brasil. Embora isso não seja considerado uma boa prática taxonômica, especialmente em um gênero tão difícil como Frankliniella. No entanto, a presença de um par de áreas glandulares no esternito abdominal III foi assinalada somente em três espécies descritas, $F$. australis Morgan, 1925, $F$. cestrum 
Moulton, 1926 e $F$. argentinae Moulton, 1935, as duas últimas sinonimizadas com $F$. australis Morgan, 1925: 137 (Nakahara, 1997), tendo em vista que os exemplares usados na descrição dessas espécies possuem, além de outras características, essa também em comum. Contudo, Frankliniella sp. n. não apresenta as cerdas PO I e apresenta 1-2 cerdas discais no esternito II, enquanto $F$. australis possui as cerdas PO I e não apresenta cerdas discais no esternito II. É uma espécie cuja relação comprimento/largura da cabeça, pronoto, metanoto e tergito IX mais próxima do valor 1. A cabeça é bem distinta, tão longa quanto larga; pronoto com cerdas am pouco desenvolvidas (1/6 do comprimento do pronoto); metanoto sem sensilo campaniforme; cerdas medianas do metanoto pouco maiores que a $1 / 2$ do comprimento do mesmo.

\subsubsection{Chave de identificação das espécies de Frankliniella}

Das 40 espécies de Frankliniella constatadas no Brasil, 18 foram descritas com base em material nativo. Embora represente quase 1/4 do total de espécies do gênero, provavelmente esse número subestima o real, pela falta de coletas e pelas já realizadas não serem representativas quanto aos hospedeiros/hábitats e à distribuição geográfica. Do total, 14 espécies não foram incluídas por várias motivos. Oito espécies relatadas foram descritas com base em um único espécimen ( $F$. bicolor, $F$. caseariae, $F$. fuscicornis, $F$. konoi e $F$. speciosa) ou em amostras muito pequenas ( $F$ : minor, $F$. nakaharai e $F$. serrata), e o caráter através do qual elas foram diferenciadas parece não ser confiável. Os registros de três espécies ( $F$. fusca, $F$. minuta e $F$. tritici), catalogadas por Silva et al. (1968), são duvidosos e foram realizados sem acompanhamento taxonômico. $F$. difficilis não foi incluída nesse estudo por falta de material para estudo. As espécies $F$. australis e $F$. setipes necessitam confirmação e estão depositadas no Seckenberg Museum, Frankfurt (SMF).

As espécies $F$. rodeos e $F$. distinguenda não podem ser separadas de $F$. gemina com segurança. Esses três nomes provavelmente representam um complexo de espécies, que somente poderá ser esclarecido através de estudos de campo detalhados das plantas hospedeiras, combinado com o exame da extensão de variação em ambos os sexos e nas ninfas. No entanto, coletas recentes revelaram novas ocorrências, especialmente de $F$. rodeos junto a $F$. zucchini, espécie vetora de vírus, o que determinou a inclusão de ambas espécies neste estudo. 


\section{Chave para as espécies de Frankliniella (fềmeas)}

1. Cerdas ocelares III raramente 1,5 vezes maior que o diâmetro longitudinal dos ocelos posteriores 2

$1^{\prime}$. Cerdas ocelares III pelo menos 2,5 vezes maiores que o diâmetro dos ocelos posteriores ...... 4

2. Corpo de coloração marrom-amarelada a marrom; asas marrom-claras com bordas mais escuras; cerdas am e aa do pronoto maiores que as cerdas discais; cerdas pm II do pronoto $\geq$ 3 vezes o comprimento do par I; tergito abdominal IX com cerdas $\mathrm{B}_{1}$ distintamente menores

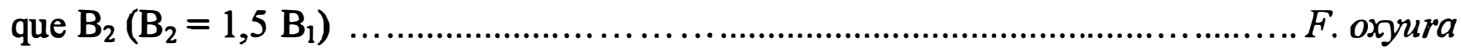

2'. Corpo de coloração amarelo claro; asas amarelo-claras; cerdas am e aa do pronoto com comprimento igual ao das cerdas discais; cerdas pm II do pronoto tão longas quanto as pm I; tergito abdominal IX com cerdas $\mathrm{B}_{1}$ aproximadamente do mesmo comprimento de $\mathrm{B}_{2}$

3. Pedicelo do segmento antenal VI constricto na base, formando um pescoço; cerdas pa mais externas do pronoto aproximadamente 2.5-3.0 vezes maiores que as pa internas, cerdas medianas do metanoto aproximadamente 0,3 vezes o comprimento do mesmo ... F. desantisi

3'. Pedicelo do segmento antenal VI sem pescoço; cerdas pa externas e internas do pronoto iguais em comprimento, mas o par mais interno mais conspícuo; cerdas medianas do metanoto aproximadamente 0,5 vezes o comprimento do mesmo

F. bertelsi

4. Olhos compostos com 5 omatídeos (2 ventrais e 3 ântero-laterais) com diâmetro maior que os omatídeos normais 5

4'. Omatideos dos olhos compostos com mesmo diâmetro 6

5. Diâmetro dos omatídeos maiores 1.5-2.0 maior que o diâmetro dos omatídeos normais; cabeça sem esculturação dorsal ao redor dos olhos; cerdas ocelares III próximas, na posição 3 ou 3/4; formato da cabeça normal, mais larga que longa (comprimento $=0,75$ largura); cerdas pm II do pronoto bem desenvolvidas, aproximadamente 4 vezes maiores que pm I; tergito VIII com pente póstero-marginal completo e irregular, com dentes pequenos; cerdas $\mathrm{B}_{1}$ do tergito IX 0,8 vezes maiores que as $\mathrm{B}_{2}$; asas anteriores uniformemente marrom claras; segmentos antenais III-V não tão longos F. fulvipennis 
5'. Diâmetro dos omatídeos maiores 3.0 vezes maiores que o diâmetro dos omatídeos normais; cabeça com esculturação dorsal ao redor dos olhos; cerdas ocelares III com inserção próxima, mas na posição 2; formato da cabeça afilando em direção à base, com comprimento semelhante à maior largura;cerdas pm II do pronoto não desenvolvidas, não nitidamente maiores que pm I; tergito VIII com pente póstero-marginal completo e regular, com dentes maiores; cerdas $B_{1}$ do tergito $\mathrm{XX} 0.8$ vezes o comprimento das $\mathrm{B}_{2}$; asas anteriores bicoloridas com base clara; segmentos antenais III-V, respectivamente, aproximadamente 6,7 e 8 vezes maiores que seu diâmetro basal F. longipennis

6. Micrópteros; metanoto com esculturação transversal estriada e paralela, com as linhas das estrias afastadas; cerdas medianas do metanoto inseridas atrás da margem anterior, na mesma direção, mas caudalmente, em relação ao par lateral; tergitos III-V com 3 pares de cerdas medianas discais longas, aproximadamente 0.8 vezes o comprimento do tergito; tergito VIII com cerdas discais medianas, aproximadamente 0,7 vezes o comprimento do tergito; pente póstero-marginal completo e irregular, com dentes pequenos e de inserção e base irregulares; cabeça levemente prolongada em frente aos olhos F. trinidadensis

6'. Macrópteros (raramente micrópteros); cerdas medianas do metanoto inseridas na margem anterior, entre o par lateral; tergitos III-V com cerdas medianas discais não alongadas; tergito VIII com cerdas discais medianas menores que 0,5 vezes o comprimento do tergito; cabeça prolongada ou não em frente aos olhos

7. Cabeça levemente prolongada em frente aos olhos; corpo de coloração amarela ...F. bondari

7'. Cabeça não prolongada em frente aos olhos; coloração do corpo variável 8

8. Asas anteriores distintamente bicoloridas, escuras com base distintamente mais claras; corpo escuro 9

8'. Asas anteriores uniformes em coloração, escuras ou claras; corpo escuro, claro ou bicolorido 14

9. Cerdas medianas do corpo, particularmente as do pronoto, exremamente desenvolvidas (am $>0,5$ vezes o comprimento do pronoto); cerdas medianas do metanoto alcançando ou quase a margem posterior do metanoto; tíbias anteriores amarelas e posteriores bicoloridas (pálida no terço basal) 
9'. Cerdas do pronoto menores que 0,5 vezes o comprimento do mesmo; cerdas medianas do metanoto não alcançando a margem posterior do metanoto; tíbias anteriores amarelas ou bicoloridas 12

10. Segmento antenal III > que 2 vezes o diâmetro da base e com cerdas desenvolvidas que alcançam a metade do segmento antenal IV

10'. Segmento antenal III $\leq$ que 2 vezes o diâmetro da base e-com cerdas que atingem apenas o $1 / 3$ basal do segmento antenal IV F. varipes

11. Tíbias medianas amarelas e posteriores marrons, com base clara; am do pronoto aproximadamente $3 / 4$ o comprimento do pronoto; tergito IX com cerdas $B_{1}$ distintamente menores que $\mathrm{B}_{2}$ F. longispinosa

11'. Tíbias medianas bicoloridas; am do pronoto pouco > que a 1/2 do comprimento do mesmo; tergito IX com cerdas $B_{1}$ iguais a $B_{2}$ F. annulipes

12. Todas as tíbias amarelas, contrastando com os fêmures marrons; cerda pós-ocular I ausente F. fulvipes

12'. Tíbias anteriores amarelas, medianas e posteriores marrons; par de cerdas pós-oculares I presentes

13. Metanoto sem sensilo campaniforme; cerdas ocelares III na posição 2, dentro do triângulo ocelar; bochechas com lados mais paralelos; segmentos antenais III e IV sem conspícua constrição apical; tergito VIII com pente póstero-marginal completo, com dentes pequenos F. hemerocallis

13'. Metanoto com sensilo campaniforme; cerdas ocelares III fora do triângulo ocelar (posição 1 ou 1/2); bochecha com lados mais arqueados, levemente constrictos posteriormente; segmentos antenais III e IV com contrição apical, formando um pescoço; tergito VIII com pente póstero-marginal com folha medianamente (falha de 2 ou 3 dentes, embora a base dos mesmos seja visível) F. insularis 
14. Cerdas ocelares III com inserção bastante próxima, na posição $3 / 4$ ou 4 , dentro do triângulo ocelar; segmento antenal VI com sensilo com base amplamente alongada na metade distal interna; tergito VIII com pente póstero-marginal completamente ausente ou quase ausente (com apenas 2 ou 3 dentes pequenos e de base larga lateralmente); metanoto sem sensilo campaniforme; coloração do corpo geralmente marrom, raramente amarela; asas amarelas F. schultzei

14'. Cerdas ocelares III com inserção afastada, próximas a margem anterior do triângulo ocelar (posição 2 ou 2/3); segmento antenal VI com sensilo com base afilada; tergito VIII com pente póstero-marginal geralmente completo e regular ou não; metanoto com sensilo campaniforme; coloração do corpo escura, clara ou bicolorida 15

15. Coloração do corpo marrom-escura 16

15'. Corpo de coloração amarelo uniforme ou amarelado com manchas marrons na porção mediana dos tergitos abdominas (bicolorido) 20

16. Cerdas PO I presentes 17

16'. Cerdas PO I ausentes 19

17. Segmento antenal III com pedicelo simples e VI com a parte distal sem constrição distinta; cerdas $B_{1}$ do tergito IX menores que $B_{2}\left(B_{1}=0,6 B_{2}\right)$; pernas marrom-escuras, exceto tarsos e tíbias anteriores (amarelo-amarronzadas ou amarronzadas) F. simplex

17'. Segmento antenal III com pedicelo diferente; cerdas $B_{1}$ aproximadamente 0,8 vezes $B_{2} \ldots 18$

18. Segmento antenal III em forma de cogumelo; segmentos antenais mais constrictos F. peruviana

18'. Segmento antenal III em forma de taça; segmentos antenais mais esbelto F. brevicaulis

19. Relação comprimento/largura das principais partes do corpo diferente de 1; cabeça com formato normal; esternito II sem cerdas discais; esternito III sem áreas glandulares; cerdas am do pronoto bastante desenvolvidas; metanoto com sensilo campaniforme; pedicelo segmento antenal III dilatado no ápice F. brunnea

19'. Relação comprimento largura das principais partes do corpo próxima a 1; cabeça alongada; esternito II com um cerda discal; esternito III com 2 áreas glandulares claras e circulares 
(uma em cada lateral); cerdas am do pronoto não tão desenvolvidas; metanoto sem sensilo campaniforme Frankliniella sp.n.

20. Pedicelo do segmento antenal III com disco expandido 21

$20^{\prime}$. Pedicelo do segmento antenal III sem disco expandido 22

21. Disco do pedicelo do segmento antenal III muito conspícuo, mais largo que a base do segmento (aproximadamente 2 vezes a largura da base do pedicelo); tergito IX sem microtríquias F. condei

21'. Disco do pedicelo do segmento antenal III distinto, mas não muito conspícuo, mais estreito que a base do segmento; tergito IX com microtríquias na metadecentral proximal F. gardeniae

22. Coloração do corpo variável; se amarela, tergitos abdominais geralmente com manchas marrons medianamente, variáveis em tamanho (desde uma pequena mancha até manchas maiores, que cobrem quase todo o tergito); tergito VIII com pente póstero-marginal completo, mas irregular, dentes medianos em comprimento F. occidentalis

22'. Corpo completamente amarelo; tergito VIII com pente póstero-marginal completo e regular, com dentes longos e finos 23

23. Esternito abdominal II com uma cerda discal (algumas vezes, duas); segmento antenal segment III amarelo F. williamsi

23'. Esternito abdominal II sem cerda discal; segmento antenal III parcialmente ou completamente marrom

24. Quatro pares de cerdas pós-oculares (par 1 ausente); segmentos antenais III-V marrons na metade apical; cerdas ocelares III inseridas entre as margens anteriores dos ocelos posteriores, separadas pela largura do ocelo anterior F. zucchini

24'. Cinco pares de cerdas pós-oculares (par 1 presente); segmentos antenais III-V quase completamente marrons; cerdas ocelares III alinhadas com a margem anterior dos ocelos posteriores ou um pouco antes e separadas por uma distância maior que a largura do ocelo anterior 25 
25. Cerdas ocelares III menos desenvolvidas, comprimento igual ao diâmetro longitudinal do ocelos posteriores, na posição $3 / 4$ ou 4 ; tergito abdominal IX com cerdas Bl levemente maiores que o comprimento médio do tergito abdominal $\mathrm{X}$ F. distinguenda

25'. Cerdas ocelares III mais desenvolvidas, comprimento maior que o diâmtro longitudinal dos ocelos posteriores, na posição $3 / 4$ ou levemente anterior (posição 2); tergito abdominal IX com cerdas $\mathrm{Bl}$ iguais ao comprimento médio do tergito abdominal

26. Cerdas am do pronoto aproximadamente $1 / 3$ do comprimento do mesmo; $\mathrm{pm}_{4}$ igual a $\mathrm{pm}_{2} \mathrm{e}$ $\mathrm{pm}_{3}$ F. gemina

26'. Cerdas am do pronoto aproximadamente $1 / 4$ do comprimento do mesmo; pm ${ }_{4}$ mais conspícua que $\mathrm{pm}_{2}$ e $\mathrm{pm}_{3}$ F. rodeos

\subsubsection{Caracterização morfológica das espécies de Frankliniella}

F. annulipes Hood, 1915 (Figura 1 das pranchas I, III, V, IX, X e XII)

Espécie grande e escura. Comprimento: aproximadamente $1,7 \mathrm{~mm}$. Coloração geral do corpo: marrom-escura, quase preta; asas bicoloridas (marrom-escuras, com o terço basal distintamente claro); tíbias anteriores amarelas, medianas e posteriores bicoloridas (base amarelo-clara e restante marrom). Segmentos antenais intermediários amarelados. Cerdas do corpo marromescuras. Pronoto com cerdas principais bastante desenvolvidas e discais pouco. Semelhante a varipes, da qual diferencia-se por apresentar tíbias medianas bicoloridas. Região Sudeste (SP). Sem importância econômica.

F. bertelsi (De Santis, 1967) (Figura 2 das pranchas I, III, V, IX, X e XII)

Comprimento do corpo: aproximadamente $1,1 \mathrm{~mm}$. Coloração geral do corpo: amarela. Pronoto: cerdas am todas pequenas e do mesmo tamanho das discais. Cerdas ocelares III pequenas. Região Sul (RS e SC). Sem importância econômica.

F. bondari Hood, 1941 (Figura 3 das pranchas I, III, V, LX, X e XII)

Comprimento: aproximadamente $1,2 \mathrm{~mm}$. Coloração geral do corpo: amarelo-claro, sem qualquer marca ou pigmentação. Asas anteriores amareladas. Cerdas principais do corpo claras. Cabeça levemente prolongada em frente aos olhos; sem cerdas PO I. Pronoto: mais largo que longo, com os cinco pares de cerdas principais desenvolvidos (am 0,2 vezes tão longa quanto o 
segmento) e 2 cerdas ântero-marginais secundárias. Metanoto reticulado na parte mediana e sem sensilos campaniformes. Tergito abdominal VIII com pente póstero-marginal incompleto medianamente (alguns lobos apenas), com 3 ou 4 microtríquias em cada lateral. Tergito abdominal LX com cerdas extremamente longas em relação ao comprimento do tergito. Região Nordeste (BA). Sem importância econômica.

F. brevicaulis Hood, 1937 (Figura 4 das pranchas I, III, V, IX, X e XII)

Comprimento do corpo: 1,2-1,5 mm. Coloração geral do corpo: marrom. Asas anteriores marrom-claras. Pernas amarelas e fêmures com sombreado marrom ao longo da superficie externa. Cabeça: mais estreita posteriormente; cerdas ocelares III na posição 1/2. Antena: pedicelo do segmento III com formato de xícara; segmento VI com a parte apical distintamente constricta. Tergito abdominal VIII com pente completo, com dentes pequenos, curtos e amplamente espaçados. Região Sudeste (ES, SP e Pariquera). Importância econômica: em bananeira (Musa sp.), danificando os frutos.

F. brunnea Priesner, 1932 (Figura 5 das pranchas I, III, V, IX, X e XII)

Foi coletada ate o momento uma única fêmea. Comprimento do corpo: 1,1 a 1,5 mm. Coloração geral do corpo: marrom-escura. Asas anteriores de coloração marrom-escura. Permas amarelasamarronzadas, fêmures distintamente sombreados de marrom ao longo da superfície externa, tíbias sombreadas mais levemente; tíbias postesriores escuras. Cabeça sem cerdas PO I, embora haja espécimens com tais cerdas presentes; cerdas ocelares III longas, na posição 1/2. Antena: pedicelo do segmento III com pequena dilatação; segmento III geralmente escuro com área basal levemente mais pálida. Pronoto: 2 ântero-marginais cerdas menores. Tergito abdominal IV sem ctenídeo. Tergito abdominal VIII com pente póstero-marginal completo, irregular e microtríquias curtas. Região Sudeste (SP). Sem importância econômica.

F. condei John, 1927 (Figura 6 das pranchas I, III, V, DX, X e XII)

Comprimento do corpo: aproximadamente $1,1 \mathrm{~mm}$. Coloração geral do corpo: amarelada. Asas anteriores claras, amareladas. Cerdas do corpo marroms. Antena: pedicelo do segmento III com forma de disco expandido e conspícuo, mais largo que a base do segmento. Metanoto com esculturação estriada transversal na parte anterior e longitudinal, nas partes mediana e posterior. Tergito abdominal VIII com pente póstero-marginal completo. Região Sudeste (MG e SP). Sem importância econômica. 
F. desantisi Sakimura \& ONeill, 1979 (Figura 7 das pranchas I, III, VI, IX, X e XII)

Comprimento do corpo: 1,3-1,5 mm. Coloração do corpo amarela, sem manchas. Antena: pedicelo do segmento III levemente dilatado; segmento antenal VI distintamente constricto na base. Cabeça sem cerdas desenvolvidas. Pronoto com comprimento próximo à largura; cerdas pa (par intemo e par externo) e pm II desenvolvidas, embora apenas pa extemas conspícuas. Metanoto com cerdas medianas afastadas da margem anterior e pouco desenvolvidas, menores que as laterais. Tergito abdominal VIII com pente póstero-marginal completo. Tergito abdominal LX com cerdas um pouco maiores que o comprimento do tergito. Região Sul (SC). Sem importância econômica.

F. distinguenda Bagnall, 1919 (Figura 8 das pranchas I, III, VI, IX, X e XII) Comprimento do corpo: aproximadamente $1,0 \mathrm{~mm}$. Coloração do corpo: amarela clara. Muito semelhante a gemina e rodeos, das quais diferencia-se por ser pouco menor e delgada. Antena: cerdas ocelares III com comprimento próximo ao diâmetro longitudinal dos ocelos posteriores, na posição $3 / 4$ ou 4 . Tergito abdominal IX com cerdas $B_{1}$ levemente maiores que o comprimento médio do tergito X. Região Sudeste (RJ e SP). Sem importância econômica.

\section{F. fulvipennis Moulton, 1933 (Figura 9 das pranchas I, III, VI, IX, X e XII)}

Comprimento do corpo: 1,4-1,5 mm. Coloracão geral do corpo: marrom. Asas anteriores uniformemente marrom-claras. Olhos compostos com 5 omatídeos (2 ventrais e 3 ânterolaterais) com diâmetro aproximadamente 1,5 a 2,0 vezes maior que o diâmetro dos omatídeos normais. Cabeça sem esculturação dorsal ao redor dos olhos; formato da cabeça mais larga que longa; cerdas ocelares III na posição 2 ou 2/3. Antena: segmento V marrom na base ou com área basal pálida pequena (menor que o terço basal). Pronoto com cerdas pm II 4 vezes maiores que pm I. Tergito abdominal IV sem ctenídeo ou ctenídeo vestigial. Tergito abdominal VIII com pente completo, irregular e com microtríquias pequenas. Segmentos antenais não tão alongados. Região Sudeste (ES e SP). Sem importância econômica.

F. fulvipes Bagnall, 1919 (Figura 10 das pranchas I, III, VI, IX, X e XIII)

Comprimento do corpo: $1,3 \mathrm{~mm}$. Coloração geral do corpo: marrom. Asas anteriores bicoloridas, marrom-escuras $\mathrm{cm}$ o quarto basal distintamente mais claro. Tíbias posteriores amarelas, algumas vezes amarronzadas. Antena: base do segmentos VI e junção dos segmentos III-V amarelos; segmento VIII 2 vezes mais longo que o VII. Tíbias e tarsos amarelos. Cabeça: mais 
estreita posteriornente; cerdas PO I ausentes. Antena: segmento VIII alongado. Tergito abdominal IV com ctenídeo vestigial. Tergito abdominal VIII com pente póstero-marginal completo, irregular e com microtríquias de medianas. Regiões Sudeste (MG) e Sul (PR e SC). Sem importância econômica.

F. gardenia Moulton, 1948 (Figura 11 das pranchas I, III, VI, LX, X e XIII)

Comprimento do corpo: 1,2 mm. Coloração geral do corpo: amarela, sem manchas escuras. Cerdas do corpo escuras. Asas anteriores claras. Assim como condei, apresenta antena com segmento II longo e pedicelo do segmento III com disco expandido. No entanto, a expansão é menor que o diâmetro basal do segmento. A base do segmento III é levemente dilatada Cabeça: cerdas ocelares III na posição2. Pronoto: com duas cerdas ântero-marginais pequenas. Tergito abdominal IV com ctenídeo vestigial. Tergito abdominal VIII com pente completo e ? longo. Tergito abdominal IX com pequenas microtríquias anteriormente. Região Sudeste (SP). Importância economica: em citros, danificando botões florais.

F. gemina Bagnall, 1919 (Figura 12 das pranchas I, III, VI, DX, X e XIII)

Comprimento do corpo: $1,5 \mathrm{~mm}$ ?. Coloração do corpo: amarela clara. Cabeça: cerdas ocelares III mais longas que o diâmetro longitudinal dos ocelos posteriores. Pronoto: cerdas am com comprimento aproximadamente $1 / 3$ do pronoto; $\mathrm{pm}_{4}$ igual a $\mathrm{pm}_{2}$ e $\mathrm{pm}_{3}$. Tergito abdominal IX com cerdas $B_{1}$ iguais ao comprimeto do tergit abdominal X. Regiões Sudeste (RJ e SP) e Sul (PR e RS). Sem importância econômica.

F. hemerocallis Crawford, 1948 (Figura 13 das pranchas I, III, VI, IX, XI e XIII)

Corpo de coloração escura. Cabeça larga, como em insularis, mas cerdas ocelares III dentro do triângulo ocelar, na posição 2. Segmentos antenais III e IV sem pescoço apical. Pronoto: cerdas submedianas (pm II) menores que o dobro do comprimento das medianas (pm I). Metanoto sem sensilo campaniforme e com retículos amplos. Tergito abdominal VIII com pente completo e dentes pequenos e de base larga. Região Sudeste (SP). Sem importância econômica.

F. insularis (Franklin, 1908) (Figura 14 das pranchas I, III, VI, IX, XI e XIII)

Comprimento do corpo: Corpo grande de coloração escura. Cabeça: larga, mais estreita posteriormente; cerdas ocelares III fora do triângulo ocelar (posição 1 ou 1/2). Antena: segmentos III e IV com prolongamento apical; segmento VI com sensilo com base ampla. Pronoto: cerdas 
submedianas (pm II) maiores que o dobro do comprimento das medianas (pm I). Metanoto com sensilo campaniforme e esculturação transversal estriada na $1 / 2$ anterior e estriada longitudinal na posterior. Tergito abdominal VIII com falha mediana. Regiões Nordeste (BA), Sudeste (ES, MG, RJ e SP) e Sul (RS e SC). Sem importância econômica.

\section{F. longipennis (Moulton, 1933) (Figura 15 das pranchas II, IV, VII, LX, XI e XIII)}

Comprimento do corpo: ao redor de $1,9 \mathrm{~mm}$. Coloração do corpo: marrom-claro. Asas anteriores bicoloridas, escuras com base clara. Cabeça: afilando posteriormente, tão longa quanto a maior largura e com esculturação ao redor dos olhos; olhos compostos com 5 omatídeos (2 ventrais e 3 ântero-laterais) aproximadamente 3 vezes maiores que os normais. Pronoto com cerdas pm II com comprimento aproximadamente igual ao de pm I. Tergito abdominal VIII com pente póstero-marginal completo e regular. Região Sudeste (ES e RJ). Sem importância econômica.

\section{F. longispinosa Moulton, 1933 (Figura 16 das pranchas II, IV, VII, LX, XI e XIII)}

Comprimento do corpo: ao redor de $1,7 \mathrm{~mm}$. Coloração geral do corpo: escura. Asas anteriores bicoloridas, escuras com base distintamente clara. As principais cerdas do corpo são marrons e excepcionalmente longas, particularmente as da cabeça e do pronoto. Tíbias anteriores e medianas amarelas e posteriores bicoloridas (marrons, com base clara). Região Sudeste (ES e MG). Sem importância econômica.

F. occidentalis (Pergande, 1895) (Figura 17 das pranchas II, IV, VII, IX, XI e XIII)

Comprimento do corpo: 1,5 mm. Embora haja espécimens com corpo de coloração variável, da amarela ao marrom-escuro, nunca são completamente amarelas. No Brasil foram constatados somente espécimens amarelos, com manchas marrons nos tergitos abdominais. Antena: pedicelo do segmento antenal III normal, sem dilatação; segmento III amarelo na base, com ápice sombreado; segmentos IV-V amarelos na base. Tíbias posteriores parcialmente amarelas. Tergito abdominal IV com ctenídeo vestigial. Tergito abdominal VIII com pente completo e irregular, dentes de comprimento mediano. Regiões Nordeste (AL e PE), Sudeste (RJ e SP) e Sul (RS). Praga principalmente de flores ornamentais, também foi constatada em pimentão e pêssego, causando danos. Sua presença no sul do País provavelmente é errônea (não há voucher specimens para esclarecimento). 
F. oxyura Bagnall, 1919 (Figura 18 das pranchas II, IV, VII, IX, XI e XIV)

Comprimento do corpo: aproximadamente 1,0 mm. Coloração geral do corpo: marrom. Cabeça: sem cerdas desenvolvidas; cerdas PO I ausentes. Pronoto: cerdas am pouco maiores que as discais, mas não conspícuas; aa (par interno e externo); pa (par interno e externo) e pm um pouco mais desenvolvidas. Metanoto: cerdas medianas com mesmo comprimento das laterais. Tergito abdominal VIII completo, com dentes longos. Tergito abdominal IX com cerdas $\mathrm{B}_{1}$ distintamente menores que $B_{2}$. Regiões Sudeste (ES, MG e RJ) e Sul (SC e PR). Sem importância econômica.

F. peruviana Hood, 1937 (Figura 19 das pranchas II, IV, VII, IX, XI e XIV)

Comprimento do corpo: aproximadamente 1,2 mm. Coloração geral do corpo: marrom-escura. Asas anteriores de coloraçao morrom-clara. Antena: pedicelo do segmento III em formato de cogumelo, mas com a parte apical retilínea; segmento VI com a parte apical distintamente constricta. Tergito abdominal VIII com pente póstero-marginal completo e irregular (regular na parte mediana). Tergito abdominal $\mathrm{XX}$ com $\mathrm{B}_{1}$ distintamente menor que $\mathrm{B}_{2}$. Região Sudeste (SP). Sem importância econômica.

F. rodeos Moulton, 1933 (Figura 20 das pranchas II, IV, VII, IX, XI e XIV)

Comprimento do corpo: aproximadamente 1,2-1,3 mm. Coloração geral do corpo: amarela. Tamanho intermediário entre $F$. distinguenda e $F$. gemina. Assim como em $F$. gemina, as cerdas ocelares III são mais longas que o diâmetro longitudinal dos ocelos posteriores e as cerdas $\mathrm{B}_{1}$ do tergito IX são tão longas quanto o comprimento do tergito abdominal $\mathrm{X}$. No entanto, as cerdas am do pronoto são menores (1/4 do comprimento do pronoto) e $\mathrm{pm}_{4}$ é mais conspícua que $\mathrm{pm}_{2} \mathrm{e}$ $\mathrm{pm}_{3}$. Segundo sua descrição, a coloração do corpo é amarelo-amarronzado claro, o crescente ocelar é marrom e os segmentos antenais intermediários III e IV são amarelos na metade distal e marrom-claros na distal e o $\mathrm{V}$ é marrom só na extremidade apical, diferenciando-se de $F$. gemina, cuja coloração é amarelo-alaranjada, crescente ocelar é vermelho-alaranjado e os segmentos antenais intermediários são mais escuros. Região Centro-Oeste (DF), Sudeste (ES, MG, RJ e SP) e Sul (RS). Sem importância econômica.

F. schultzei (Trybom, 1910) (Figura 21 das pranchas II, IV, VII, IX, XI e XIV)

Comprimento do corpo: ao redor de 1,5 mm. Coloração geral do corpo: marrom. Asas anteriores claras. A forma clara de $F$. schultzei, ainda não constatada no Brasil, não foi incluída na chave, 
mas apresenta as mesmas características morfológicas da forma escura. Cabeça: cerdas ocelares III com inserção próxima, na poisição $3 / 4$ ou 4 , dentro do triângulo ocelar. Segmento antenal VI com sensilo de base ampla na metade distal interna do segmento. Metanoto sem sensilo campaniforme. Tergito VIII com pente póstero-marginal totalmente ausente ou quase (2 ou 3 dentes pequenos e de base larga em cada lateral). Regiões Nordeste (BA, MA, PE e RN), Centro-Oeste (GO e MS), Sudeste (SP) e Sul (PR e RS). Importância econômica: praga de várias culturas, incluindo algodoeiro, melancia, alface e tomateiro. É vetora de Tospovirus.

\section{F. simplex Priesner, 1924 (Figura 22 das pranchas II, IV, VII, IX, XI e XIV)}

Comprimento do corpo: 1,4 mm grande. Coloração do corpo: marrom-escura. Cabeça: cerdas ocelares III na posição 2/3. Antena: pedicelo do segmento III simples, segmento III marrom ou marrom-amarelado, segmento VI com a parte distal sem constrição distinta. Pronoto: cerdas am aproximadamente $1 / 3$ do comprimento do pronoto. Tergito abdominal IV com ctenídeo. Tergito abdominal VIII com pente póstero-marginal completo e com microtríquias medianas. Tergito IX: cerdas $B_{1}$ pequenas, menores que o comprimento do segmento. Regiões Sudeste (SP). Sem importância econômica.

\section{F. trinidadensis Hood, 1941(Figura 23 das pranchas II, IV, VIII, IX, XI e XIV)}

Comprimento do corpo: 1,0 mm. Coloração geral do corpo: amarela. Cabeça: levemente prolongada em frente aos olhos; cerdas ocelares III na posição II. Antena: pedicelo do segmento III levemente dilatado. Micróptera (lobos das asas anteriores menores que $0,4 \mathrm{~mm}$, nas fêmeas). Pronoto: com 4 cerdas ântero-marginais pequenas, quase sem a presença de cerdas discais. Metanoto: sem sensilo campaniforme; esculturação com estrias transversais amplamente espaçadas; inserção das cerdas medianas afastadas da margem anterior, caudalmente e quase na mesma direção (um pouco mais intema) que as laterais. Ctenídeos abdominais não desenvolvidos. Tergitos abdominais com 3 pares de cerdas longas, aproximadamente 0,65 vezes tão longas quanto o segmento. Tergito abdominal VIII com pente póstero-marginal completo, irregular e atípico. Tergito abdominal $\mathrm{XX}$ com cerdas $\mathrm{B}_{1}$ distintamente menores que $\mathrm{B}_{2}$. Região Sudeste (SP). Sem importância econômica.

F. varipes Moulton, 1933 (Figura 24 das pranchas II, IV, VIII, IX, XI e XIV)

Comprimento do corpo: aproximadamente $1,4 \mathrm{~mm}$. Coloração geral do corpo: escura e asas bicoloridas. Tíbias medianas completamente amarelas. Cabeça com constrição posterior?; cerdas 
ocelares III na posição 2/3. Pronoto: cerdas principais longas e quase sem cerdas discais. Metanoto: com cerdas longas. Pente póstero-marginal completo e com microtríquias medianas em comprimento. Tergito IV com par de ctenídeos bem desenvolvidos. Tergito VIII: pente completo, com dentes finos, de bases largas e amplamente espeçados. Tergito IX com cerdas $\mathrm{B}_{1}$ menores que $B_{2}$. Região Sudeste (ES, RJ e SP). Sem importância econômica.

F. williamsi Hood, 1915 (Figura 25 das pranchas II, IV, VIII, IX, XI e XIV)

Comprimento do corpo: aproximadamente $1,1 \mathrm{~mm}$. Coloração geral do corpo: amarela. Antena: segmento VII pelo menos 2,5 vezes tão longo quanto largo. Esternito abdominal II com 1 ou 2 cerdas discais. Tergito abdominal VIII: pente póstero-marginal completo e com dentes longos. Tergito abdominal IX: cerdas $\mathrm{B}_{1}$ distintamente menores que $\mathrm{B}_{2}\left(\mathrm{~B}_{1}\right.$ maior que 110 microns; $\mathrm{B}_{2}$ maior que 130 microns). Região Sudeste (MG e SP) e Sul (PR e SC). Importância econômica: em milho.

F. zucchini Nakahara \& Monteiro, 1999 (Figura 26 das pranchas II, IV, VIII, IX, XI e XIV)

Comprimento do corpo: 1,2-1,4 mm. Coloração do corpo: amarela. Asas anteriores amarelas. Cerdas do corpo marrom. Cabeça: cerda PO I ausente; cerdas ocelares III inseridas entre as margens anteriores dos ocelos posteriores, separadas pela largura do ocelo anterior. Tergito VIII com pente completo e com microtríquias medianos. Semelhante à gemina, rodeos e distinguenda, das quais diferencia-se pela ausência das cerdas PO I, pela coloração dos segmentos antenais III-V e pela posição das cerdas ocelares III e a distância entre suas bases. Regiões Sudeste (SP) e Centro-Oeste (DF). Importância econômica: vetora do Tospovirus ZLCV (zucchini lethal chlorosis virus) em abobobrinha, causadora da doença ZLD (zucchini lethal chlorosis) . 

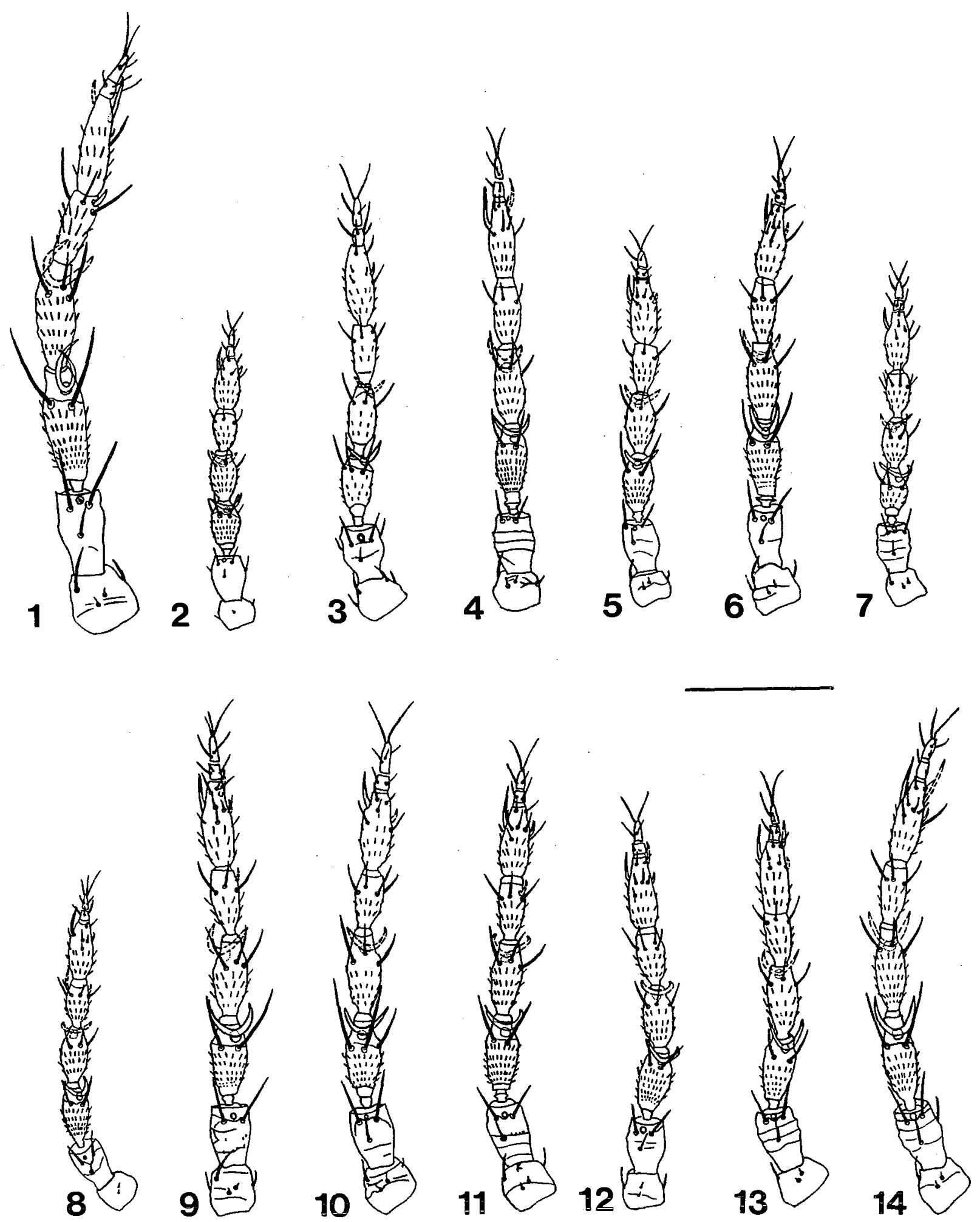

Prancha I. Figuras 1-14. Antena direita da 9 de Frankliniella spp. 1. F. annulipes; 2. F. bertelsi; 3. F. bondari; 4. F. brevicaulis; 5. F. brunnea; 6. F. condei; 7. F. desantisi; 8. F. distinguenda; 9. F. fulvipennis; 10. F. fulvipes; 11. F. gardenia; 12. F. gemina; 13. F. hemerocallis; 14. F. insularis. 

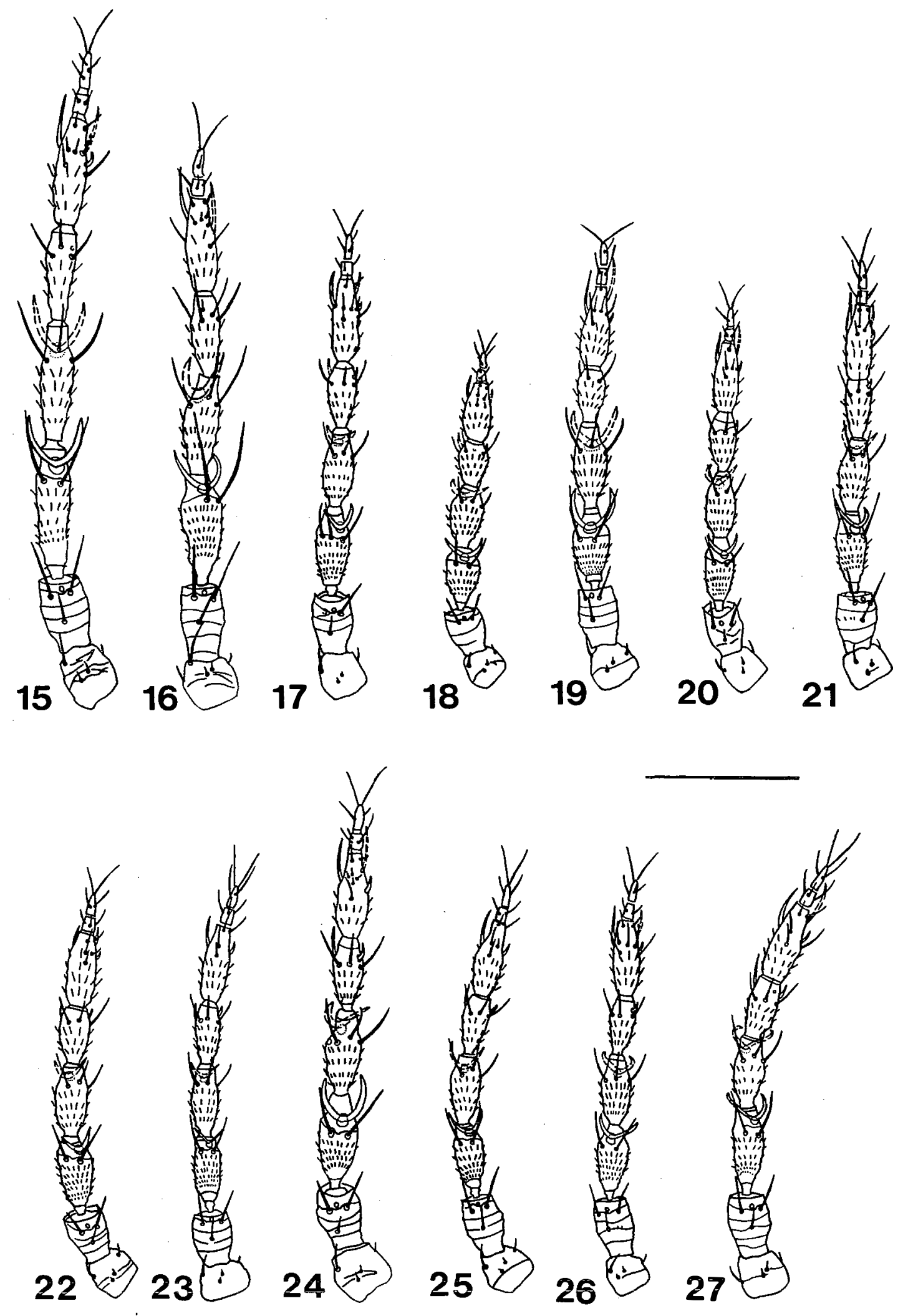

Prancha II. Figuras 15-27. Antena direita da $q$ de Frankliniella spp. 15. F. longipennis; 16. F. longispinosa; 17. $F$. occidentalis; 18. $F$. oxyura; 19. F. peruviana; 20. $F$. rodeos; $21 . F$. schultzei; 22. F. simplex; 23. F. trinidadensis; 24. F. varipes; 25. F. williamsi; 26. F. zucchini; 27. Frankliniella sp. n. 

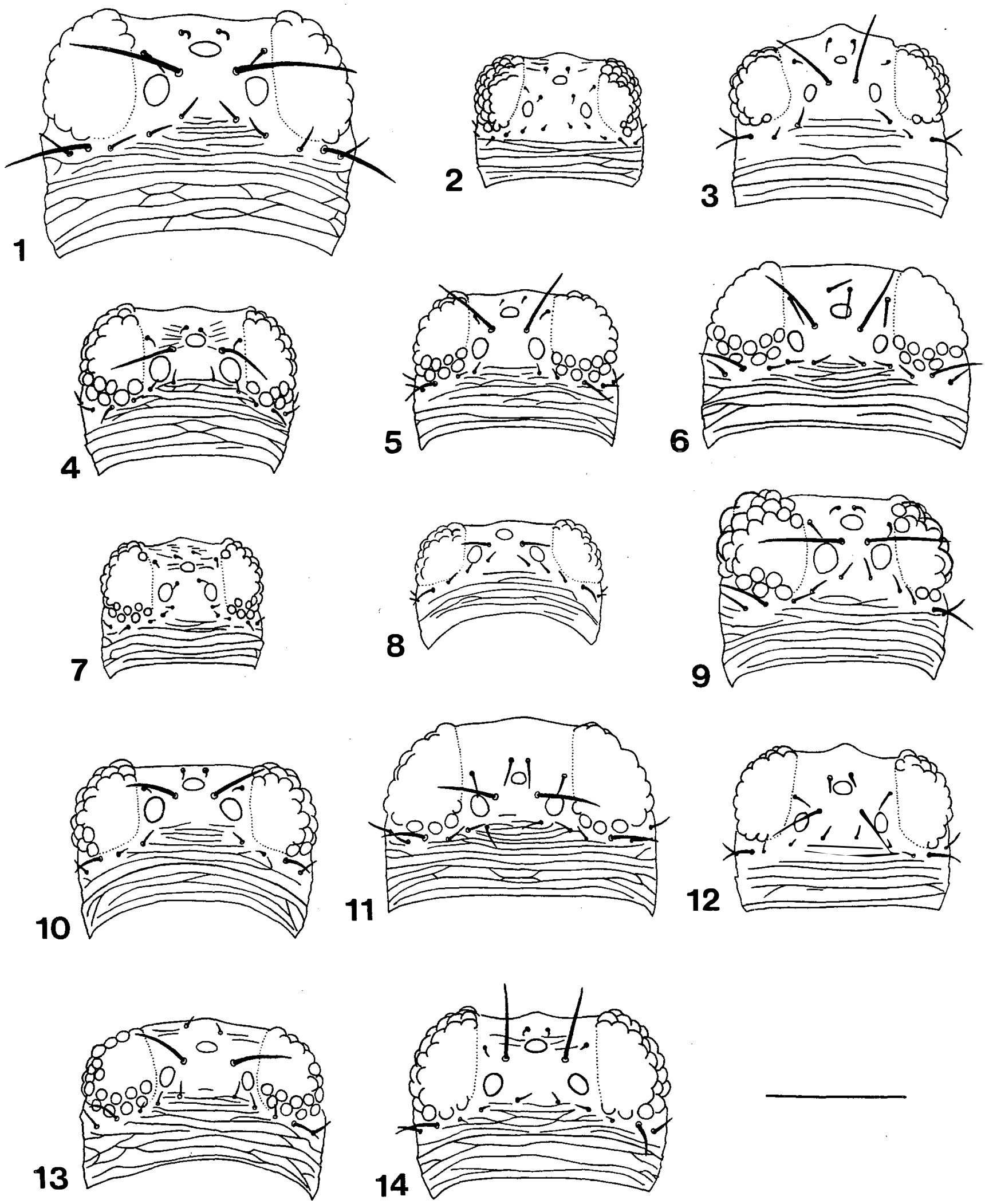

Prancha III. Figuras 1-14. Cabeça (dorsal) da + de Frankliniella spp. 1. F. annulipes; 2. F. bertelsi; 3. F. bondari; 4. F. brevicaulis, 5. F. brunnea, 6. F. condei; 7. F. desantisi; 8. F. distinguenda; 9. F. fulvipennis; 10. F. fulvipes; 11. F. gardenia; 12. F. gemina; 13. F. hemerocallis; 14. F. insularis. 

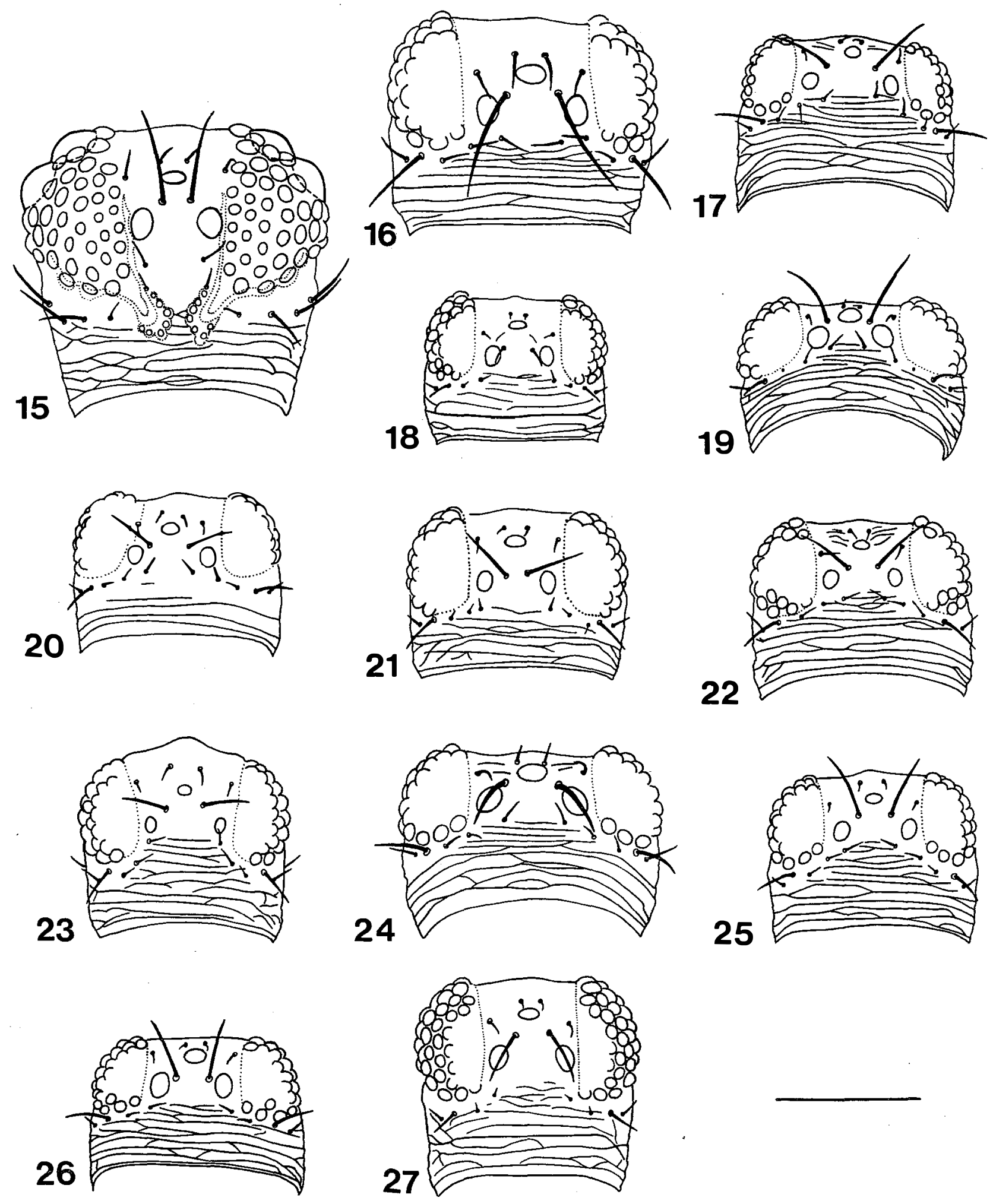

Prancha IV. Figuras 15-27. Cabeça (dorsal) da of de Frankliniella spp. 15. F. longipennis; 16. F. longispinosa; 17. F. occidentalis; 18. $F$. oxyura; 19. F. peruviana; 20. F. rodeos; $21 . F$. schultzei; 22. F. simplex; 23. F. trinidadensis; 24. F. varipes; 25. F. williamsi; 26. F. zucchini; 27. Frankliniella sp. n. 

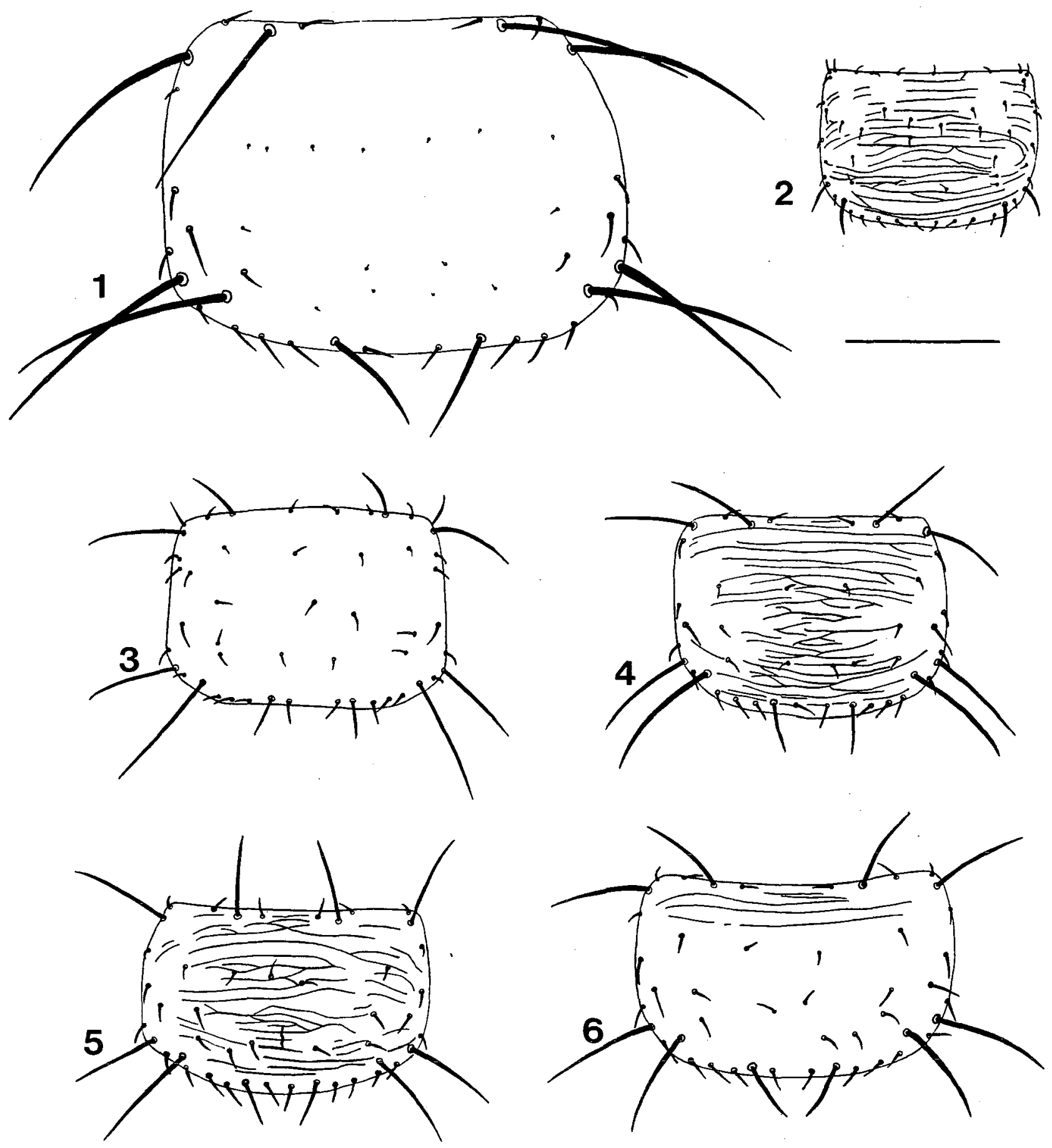

Prancha V. Figuras 1-6. Pronoto da + de Frankliniella spp. 1. F. annulipes; 2. F. bertelsi; 3. F. bondari; 4. F. brevicaulis; 5. F. brunnea; 6. F. condei. 

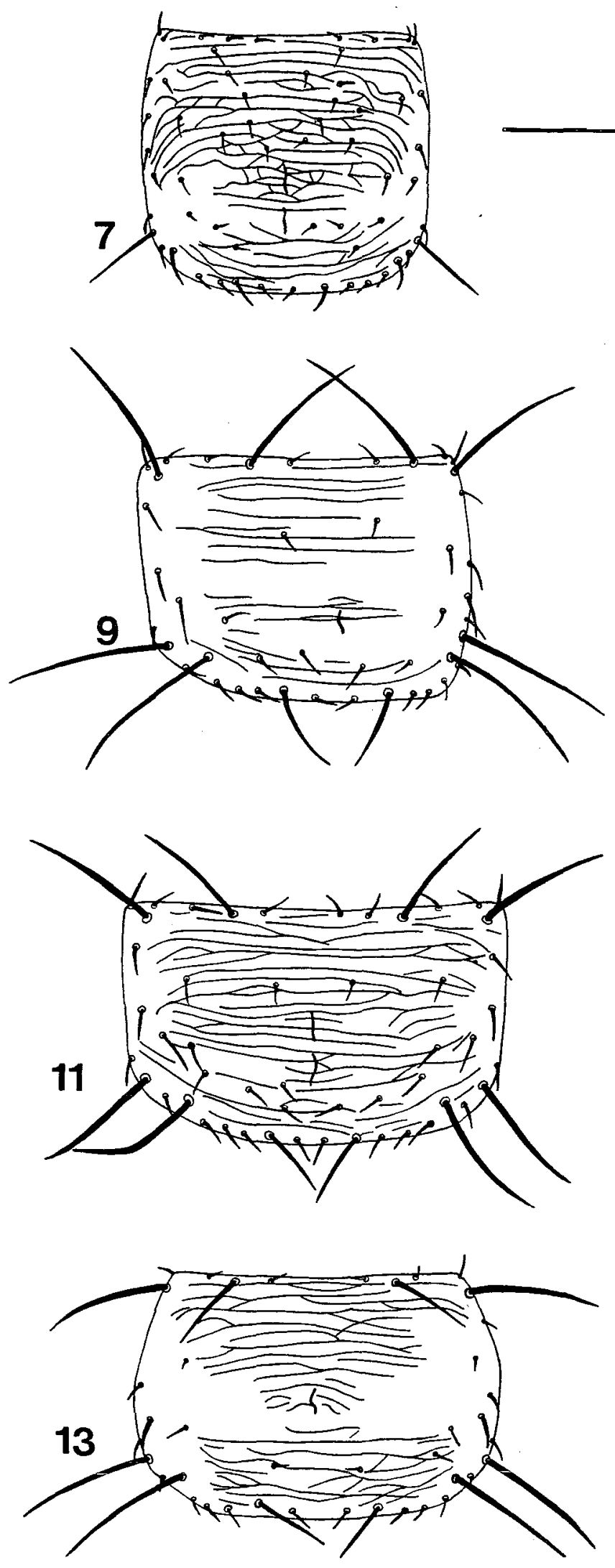
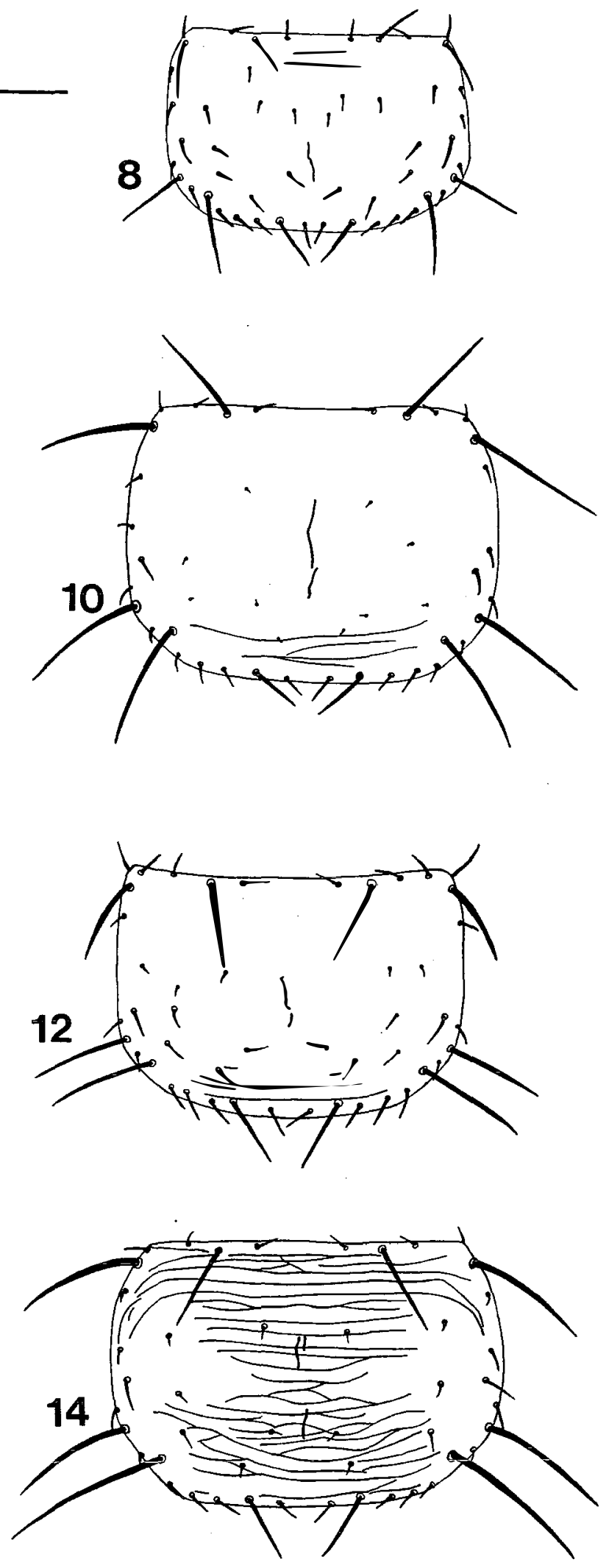

Prancha VI. Figuras 7-14. Pronoto da 9 de Frankliniella spp. 7. F. desantisi; 8. F. distinguenda; 9. F. fulvipennis; 10. F. fulvipes; 11. F. gardenia; 12. F. gemina; 13. F. hemerocallis; 14. F. insularis. 


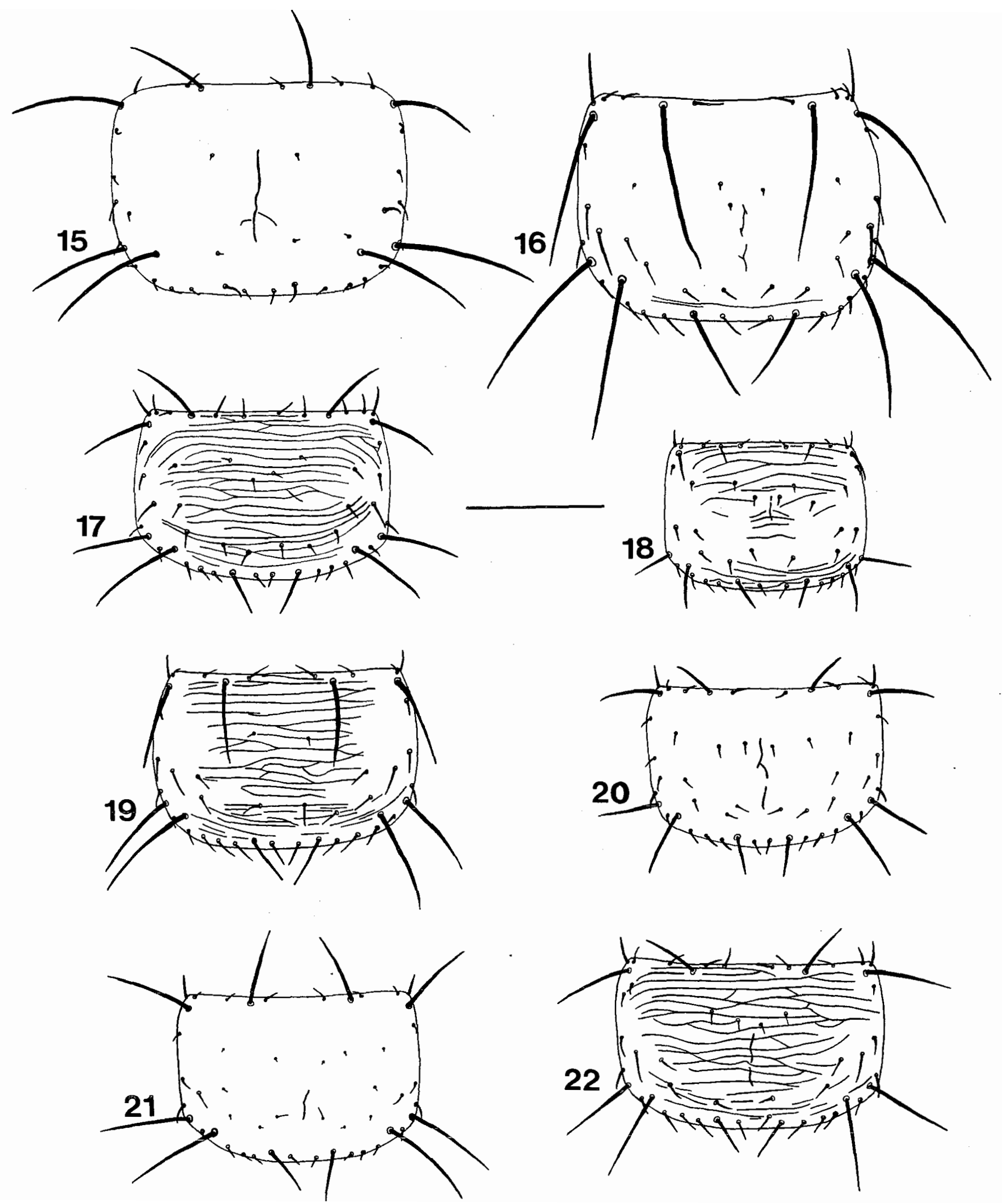

Prancha VII. Figuras 15-22. Pronoto da + de Frankliniella spp. 15. F. longipennis; 16. F. longispinosa; 17. F. occidentalis; 18. F. oxyura; 19. F. peruviana; 20. F. rodeos; 21. F. schultzei; 22. F. simplex. 

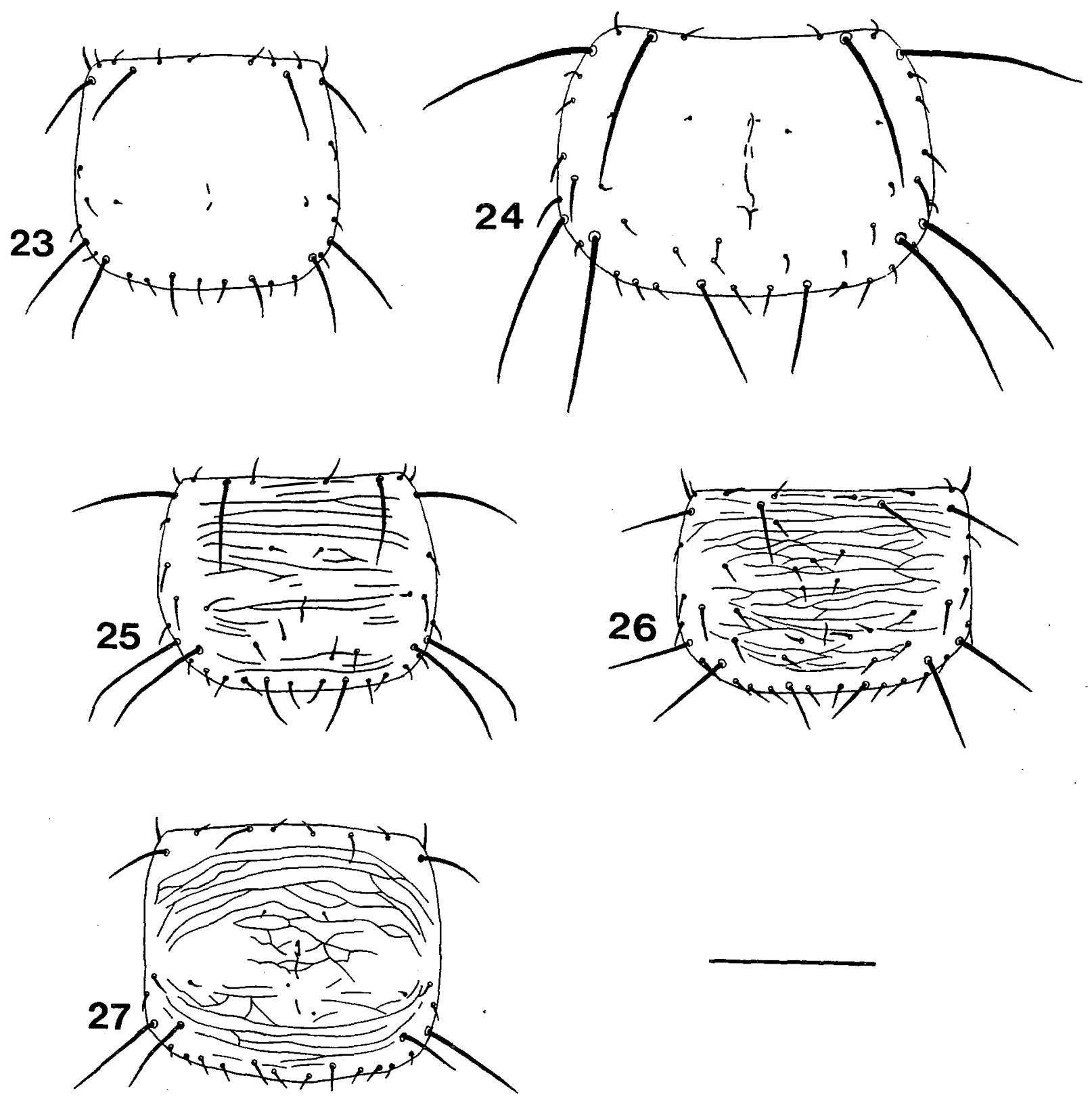

Prancha VIII. Figuras 23-27. Pronoto da + de Frankliniella spp. 23. F. trinidadensis; 24. F. varipes; 25. F. williamsi; 26. F. zucchini; 27. Frankliniella sp. n. 

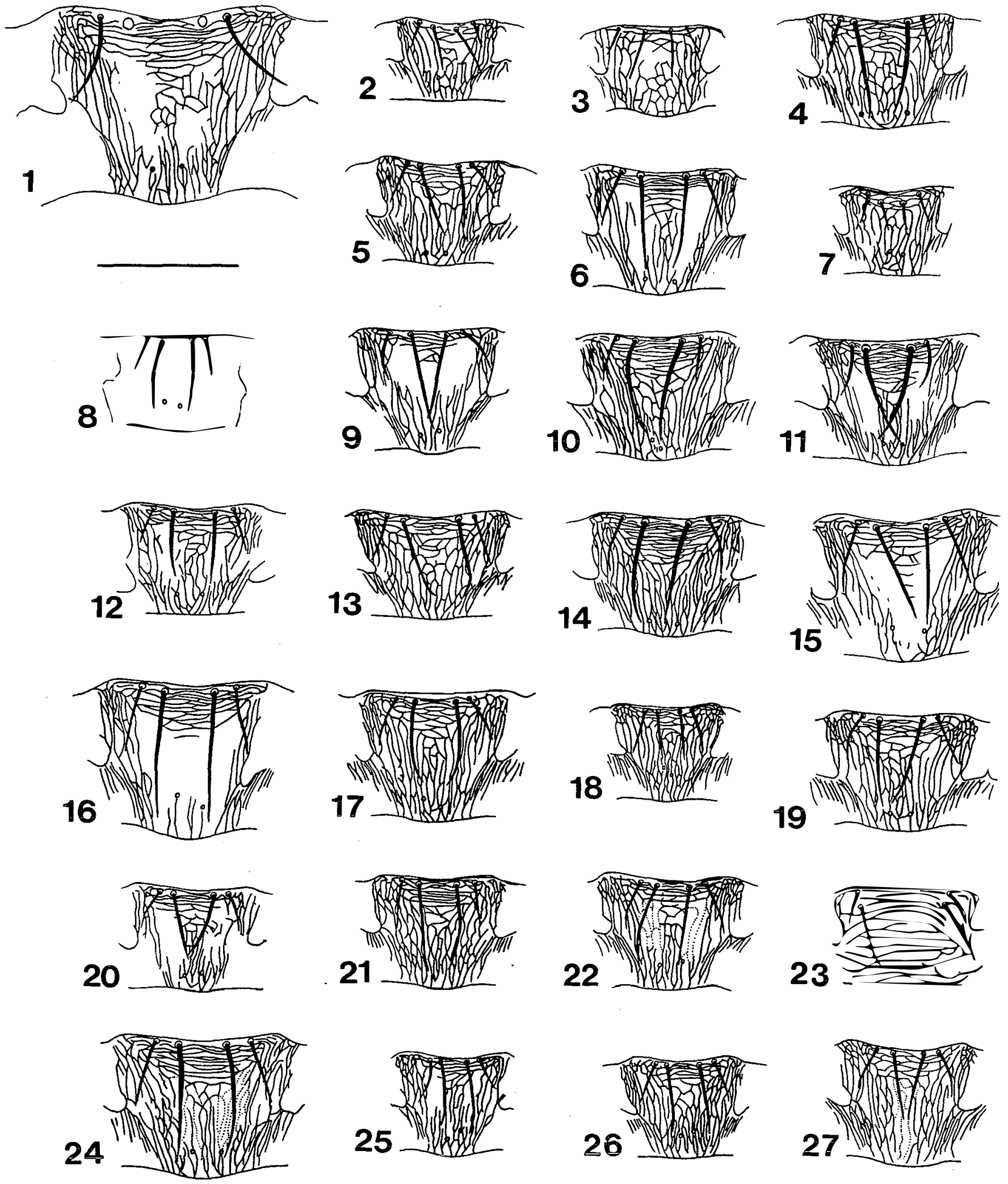

Prancha IX. Figuras 1-27. Metanoto da + de Frankliniella spp. 1. F. annulipes; 2. F. bertelsi; 3. F. bondari; 4. F. brevicaulis; 5. F. brunnea; 6. F. condei; 7. F. desantisi; 8. F. distinguenda; 9. F. fulvipennis; 10. F. fulvipes; 11. F. gardenia; 12. F. gemina; 13. F. hemerocallis; 14. F. insularis; 15. F. longipennis; 16. F. longispinosa; 17. F. occidentalis; 18. F. oxyura; 19. F. peruviana; 20. F. rodeos; 21. F. schultzei; 22. F. simplex; 23. F. trinidadensis; 24. F. varipes; 25. F. williamsi; 26. F. zucchini; 27. Frankliniella sp. n. 

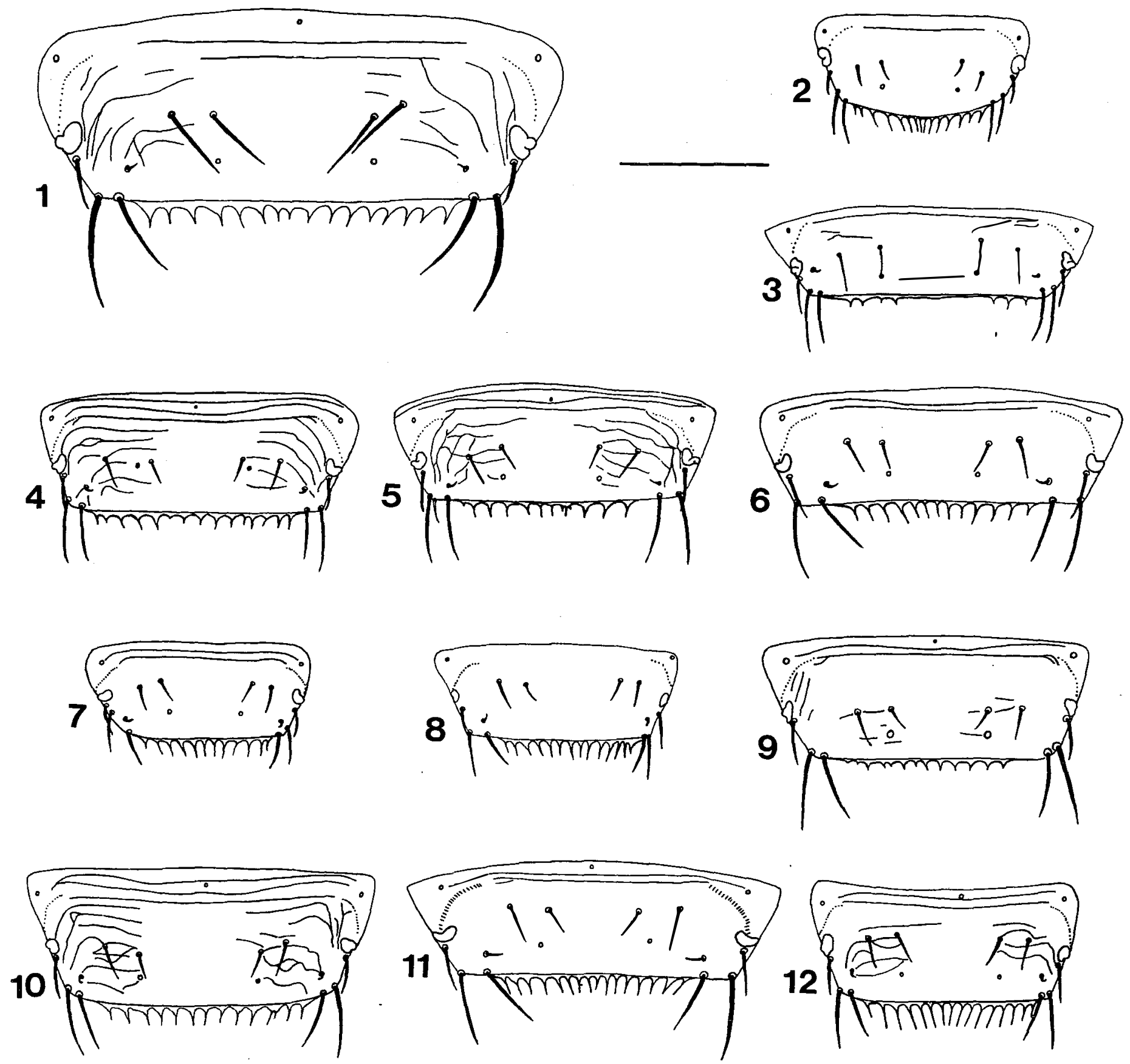

Prancha X. Figuras 1-12. Tergito abdominal VIII da $q$ de Frankliniella spp. 1. F. annulipes; 2. F. bertelsi; 3. F. bondari; 4. F. brevicaulis; 5. F. brunnea; 6. F. condei; 7. F. desantisi; 8. F. distinguenda; 9. F. fulvipennis; 10. F. fulvipes; 11. F. gardenia; 12. F. gemina. 

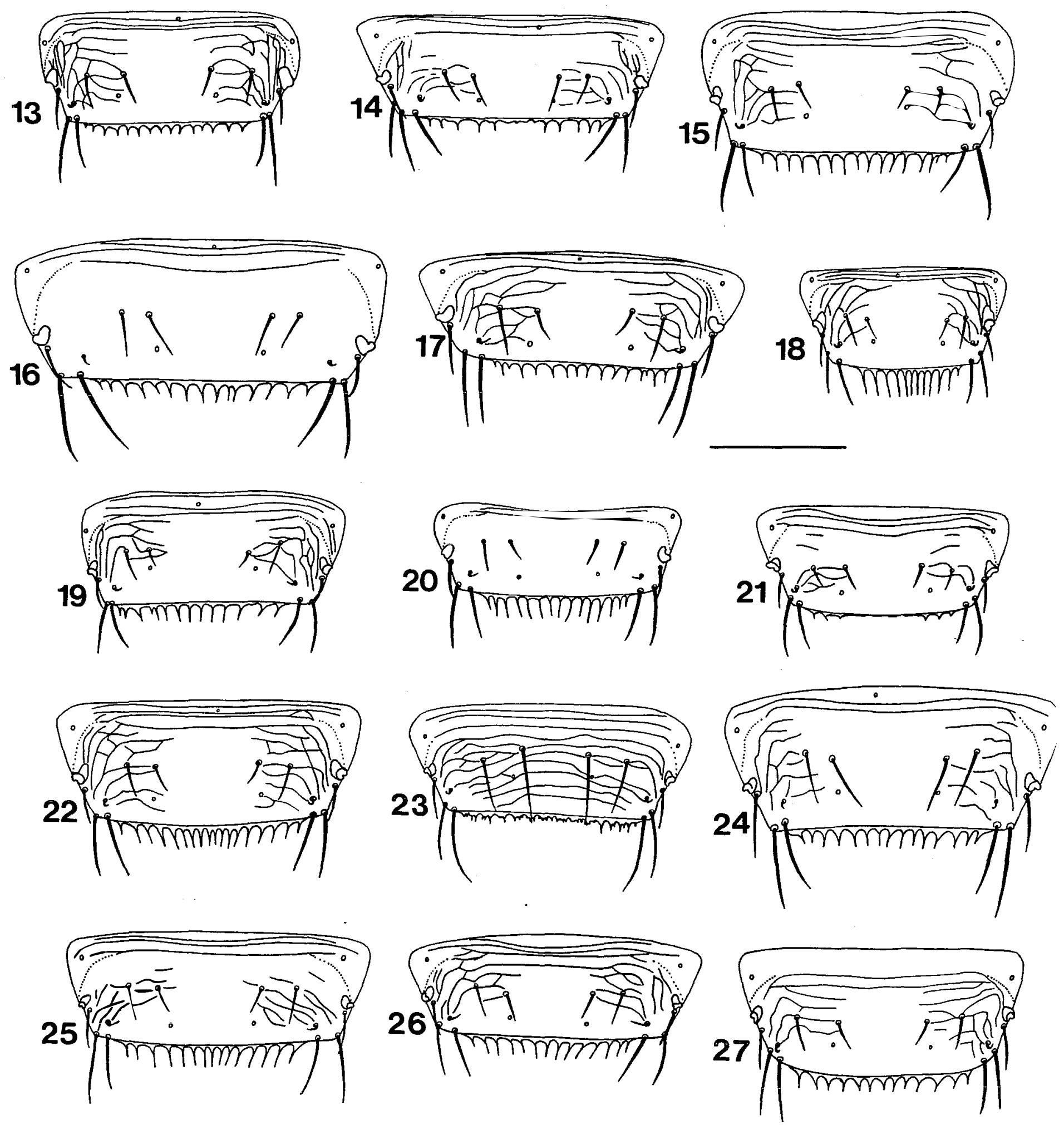

Prancha XI. Figuras 13-27. Tergito abdominal VIII da 9 de Frankliniella spp. 13. F. hemerocallis; 14. F. insularis; 15. F. longipennis; 16. F. longispinosa; 17. F. occidentalis; 18. F. oxyura; 19. F. peruviana; 20. F. rodeos; 21. F. schultzei; 22. F. simplex; 23. F. trinidadensis; 24. F. varipes; 25. F. williamsi; 26. F. zucchini; 27. Frankliniella sp. n. 


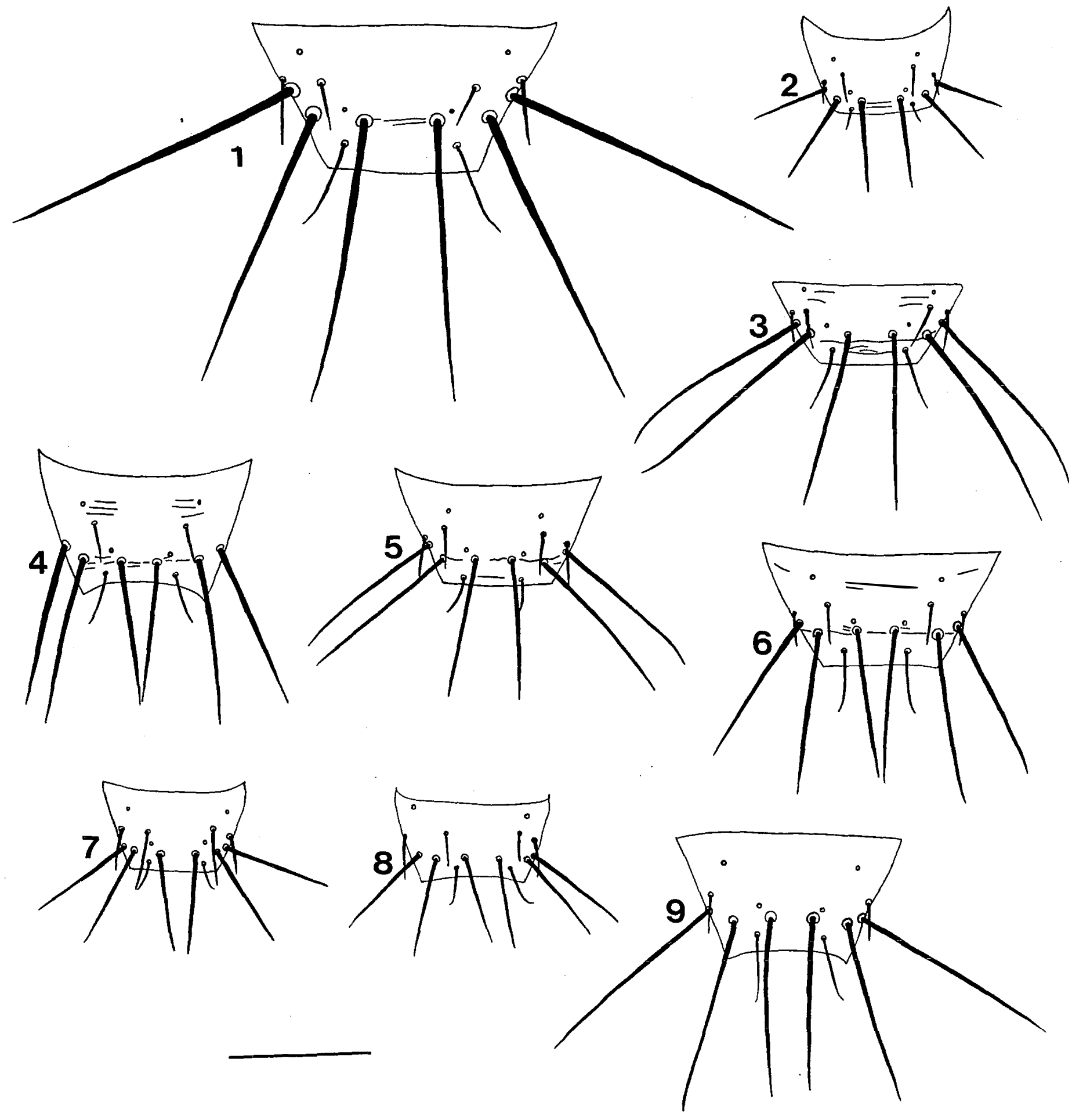

Prancha XII. Figuras 1-9. Tergito abdominal IX da $९$ de Frankliniella spp. 1. F. annulipes; 2. F. bertelsi; 3. F. bondari; 4. F. brevicaulis; 5. F. brunnea; 6. F. condei; 7. F. desantisi; 8. F. distinguenda; 9. F. fulvipennis. 

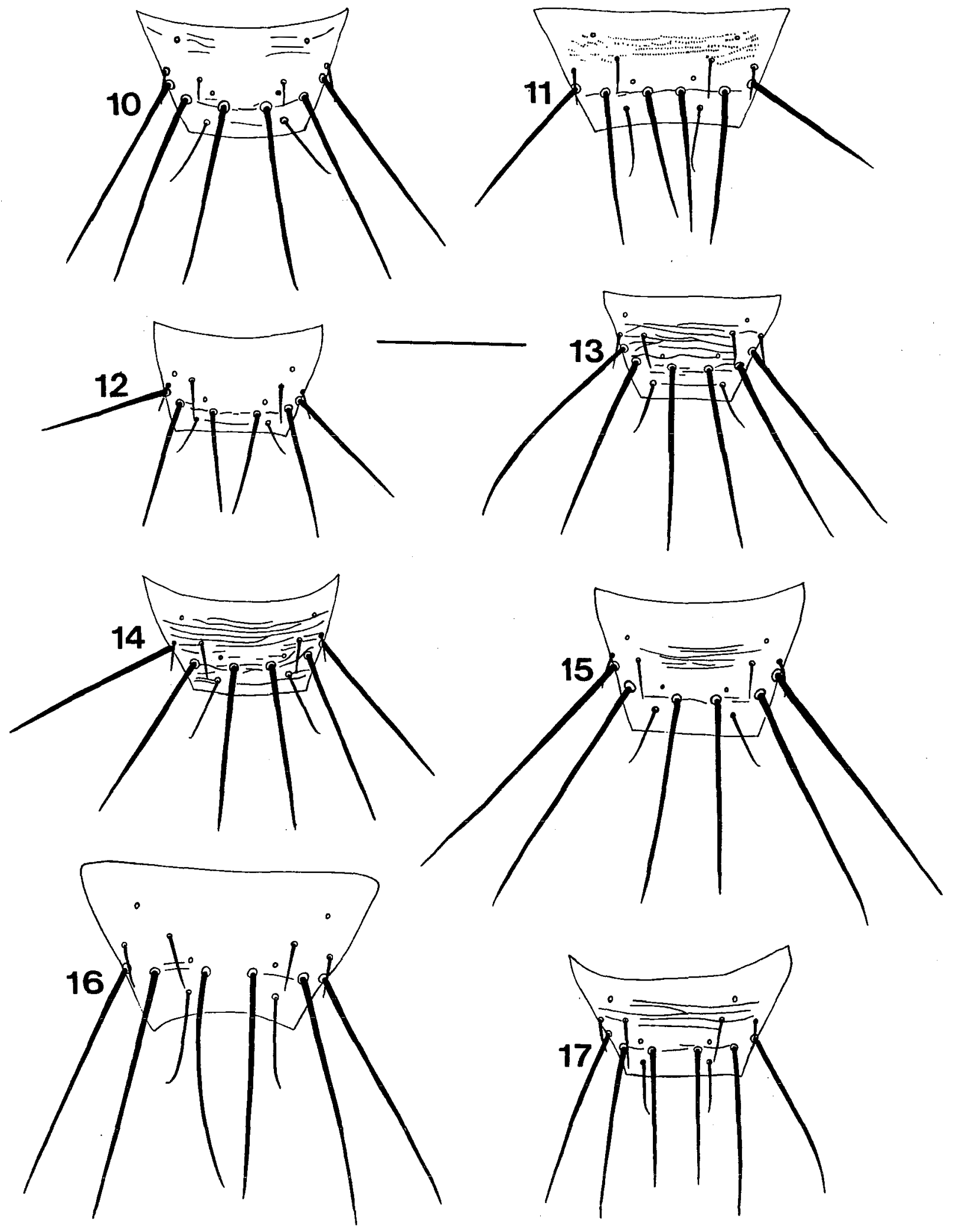

Prancha XIII. Figuras 10-17. Tergito abdominal IX da $\odot$ de Frankliniella spp. 10. F. fulvipes; 11. F. gardenia; 12. F. gemina; 13. F. hemerocallis; 14. F. insularis; 15. F. longipennis; 16. F. longispinosa; 17. F. occidentalis. 

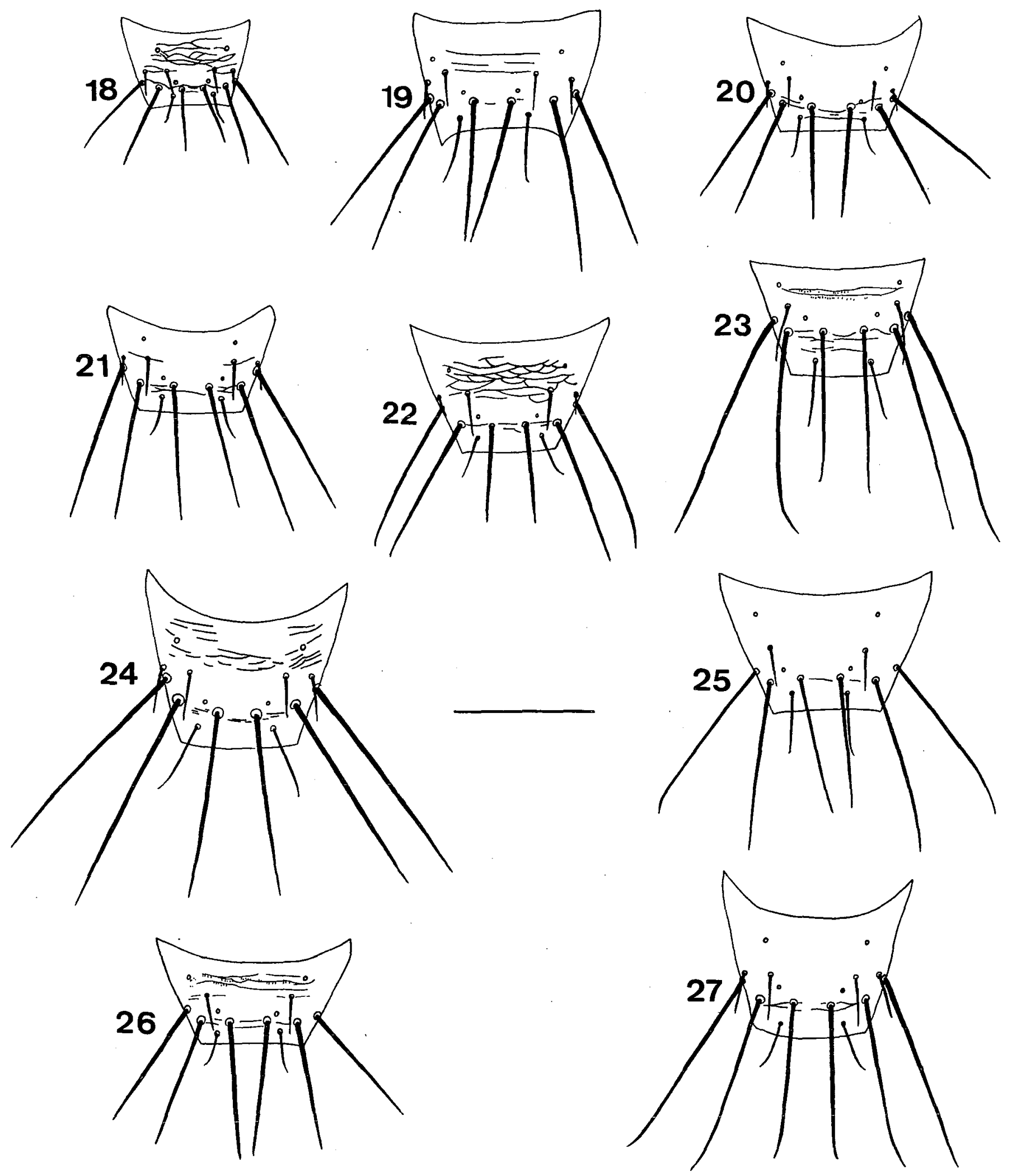

Prancha XIV. Figuras 18-27. Tergito abdominal IX da $q$ de Frankliniella spp. 18. F. oxyura; 19. F. peruviana; 20. F. rodeos; 21. F. schultzei; 22. F. simplex; 23. F. trinidadensis; 24. F. varipes; 25. F. williamsi; 26. F. zucchini; 27. Frankliniella sp. n. 


\section{O GÊNERO Thrips NO BRASIL}

\section{RESUMO}

O gênero Thrips é representado no Brasil por quatro espécies, três delas de importância agrícola e uma associada a flores de Eucalyptus spp. Uma chave para as espécies é apresentada, com comentários sobre a caracterização morfológica.

\section{SUMMARY}

The genus Thrips is represented by four species in Brazil, three of them are considered important as crop pests and one is associated with Eucalyptus flowers. A key to these species is presented, with comments on their morphological characters.

\subsection{Introdução}

O gênero Thrips apresenta aproximadamente 280 espécies (Nakahara, 1994), na sua maioria da região Holoártica e do Velho Mundo. Nenhuma das espécies de Thrips é endêmica ao sul do México, sendo T. addendus a única espécie nativa do México. Na América do Norte, há cerca de 62 espécies (43 endêmicas) (Mound \& Marullo, 1996). Apenas quatro espécies foram relatadas no Brasil: T. australis (Bagnall, 1915: 592), T. palmi Karny, 1925, T. simplex (Morison, 1930) e T. tabaci Lindeman, 1889 (Nakahara, 1994; Mound \& Marullo, 1996, Monteiro et al., 1995; Monteiro et al., 1999). Thrips minutus v. puttemansi Lima (1926) e Thrips saccharoni Moulton (1933), apesar de também registradas, representam uma espécie de outro gênero, Stenchaetothrips minutus (Deventer, 1906) (Nakahara, 1994). Thrips oryzae Williams, 
1916 foi transferido para outro gênero, sendo o nome válido atualmente Stenchaetothrips oryzae. Embora não seja em sua maioria um gênero do Novo Mundo, possivelmente o número reduzido de espécies relatadas no Brasil esteja relacionado à ausência de levantamentos.

0 gênero Thrips caracteriza-se por apresentar: antena com 7 ou 8 segmentos (7 na maioria das espécies); segmentos antenais III e IV com sensilos em forma de furca; cabeça sem cerdas ocelares I, cerdas ocelares II menores que as III, cerdas ocelares III em posição 1 ou 2; olhos compostos geralmente sem omatídeos coloridos (exceto em duas espécies); pronoto com 2 pares de cerdas póstero-angulares longas e 3 pares de cerdas póstero-marginais; asas anteriores geralmente com a primeira nervura de cerdas interrompida, com 2-8 cerdas distais, nervura posterior com a nervura de cerdas completa, escama geralmente com 5 cerdas marginais e 1 discal; tergito II com 3 ou 4 cerdas laterais; tergitos V-VIII com um ctenídeo em cada lateral, no VIII em posição póstero-mediana em relação ao espiráculo; esternito I com cerdas ânteromedianas presentes ou não (Nakahara, 1994).

\subsection{Material e Métodos}

As observações morfológicas foram realizadas nos espécimens montados em lâminas de microscopia das coleções da ESALQ/USP (Entomologia). No caso de exemplares não montados em lâminas, inicialmente fez-se o preparo e a montagem dos espécimens, segundo o método descrito em Mound \& Ptikin, (1972) e Mound \& Marullo (1996), para posterior observação. As espécies de Thrips examinadas pertencem à coleção da ESALQ/USP (Entomologia) (Apêndice 3).

\subsection{Resultados e Discussão}

Das quatro espécies de tripes constatadas no Brasil, três são pragas de uma ou mais culturas e T: australis encontra-se associada a flores de Eucalyptus spp. T. simplex é uma praga de gladíolo (palma de Santa Rita) e encontra-se amplamente dispersa pelo mundo. Além dos danos diretos que causam, $T$ : palmi e $T$ : tabaci são polífagas e também vetoras de vírus. T. tabaci parece ter preferência por lilliáceas (alho, aspargo, cebola e cebolinha) e, embora tenha sido comumente relatada como praga do algodoeiro na literatura agrícola brasileira, nenhum 
indivíduo foi coletado recentemente na cultura (Monteiro et al., 1998). Representando uma das espécies mais importantes para a Horticultura atualmente, $T$. palmi está presente no Estado de São Paulo pelo menos desde 1992 e hoje encontra-se disperso por várias localidades, tendo sido constatado em todas as regiões geográficas brasileliras, em várias culturas, mas principalmente em solanáceas (batata) e cucurbitáceas (melancia, melão) pimentão, berinjela e feijão (Monteiro et al., 1995; Monteiro et al., 1999 ; Monteiro 2000)

A diferenciação das fêmeas de T. australis, T. palmi, T. simplex e T' tabaci é baseada principalmente em caracteres morfológicos simples e de fácil visualização, como a primeira nervura da asa anterior e o número de segmentos antenais, mas também em outros caracteres que dependem de um preparo adequado e uma boa montagem, como a inserção das cerdas ocelares III, número de cerdas laterais no tergito abdominal II e número de póros no IX. Ainda, há alguns caracteres distintivos, mas visíveis mais facilmente em material recém-coletado ou bempreservado, como a coloração do crescente ocelar e do corpo.

\subsubsection{Chave para as espécies de Thrips}

\section{Chave de identificação das espécies de Thrips (fềmeas)}

1. Primeira nervura da asa anterior quase completa (sem internupação ma fileira de cerdas) T. australis

1'. Primeira nervura da asa anterior incompleta (interrompida)

2. Antena com 8 segmentos; corpo de coloração marrom-escura; metanoto com esculturação reticulada; asa anterior com 5-8 cerdas discais T. simplex

2'. Antena com 7 segmentos; corpo de coloração amarela, amarela-amarronzada ou marrom; esculturação do metanoto diferente; asa anterior com 2-4 cerdas discais 3

3. Cerdas ocelares III fora do triângulo ocelar; tergito abdominal II com 4 cerdas marginais; pleurotergitos abdominais sem microtríquias; crescente ocelar vermelho e corpo de coloração amarela T. palmi 
3'. Cerdas ocelares III dentro do triângulo ocelar; tergito abdominal II com 3 cerdas marginais; pleurotergitos abdominas com numerosas fileiras de microtriquias; crescente ocelar acinzentado e corpo de coloração amarela ou amarela-amarronzada T. tabaci

\subsubsection{Caracterização morfológica das espécies de Thrips}

Thrips australis. Comprimento do corpo: 1,4 a 1,6 mm. Coloração do corpo: amarela e marrom; parte posterior da cabeça e região ocelar marrons; tórax marrom e amarelo; parte mediana dos tergitos abdominas I-VIII, todo tergito IX e $\mathrm{X}$ marrons; pernas amareladas; asas anteriores claras; cerdas do corpo escuras; crescente ocelar vermelho; antena marrom, exceto 1/2 basal do segmento III e parte basal dos segmentes IV e V, que são amarelos. Segmentos antenais III, IV e VI com lados paralelos; cerdas curtas, mas conspícuas no pronoto e asas anteriores; asas anteriores com primeira nervura de cerdas quase completa; metanoto reticulado poligonalmente; numerosas cerdas acessórias nos estemitos e pleurotergitos abdominais.

Thrips palmi. Comprimento do corpo: 1,0 a 1,2 mm. Coloração do corpo: amarelo-dourado; crescente ocelar vermelho; asas anteriores claras, amareladas; cerdas escuras (marrons). Antena com segmento I amarelo-claro, II amarelo mais escuro, III amarelo nos 2/3 basais e marrom no 1/3 apical, IV e V amarelos na metade basal e marrons na apical, VI marrom, com a base clara algumas vezes, e VIII marrom. Abdome completamente amarelo, cerdas principais marrons, crescente ocelar vermelho, cerdas ocelares III fora do triângulo ocelar, na posição 1 ou $1 / 2$, metanoto com escultura convergendo posteriormente, tergito abdominal II com quatro cerdas laterais, tergito VIII com pente póstero-marginal completo, esternito I com três 3 cerdas ântero medianas.

Thrips simplex. Comprimento do corpo: 1,5 - 1,8 mm. Coloração do corpo: marrom-escura; pernas marrons, exceto os tarsos, tibías anteriores e ápice das tíbias medianas e posteriores, e base dos fềmures, que são marrom-amarelados; crescente ocelar vermelho; asas anteriores marrom-claras, com o 1/4 basal distintamente mais claro; cerdas escuras; antena marrom, exceto a extremidade apical do II e o todo segmento III, que são marrons-amarelados. Asa anteriores com 5-8 cerdas distais na primeira nervura, antena com 8 segmentos e segmento III marrom- 
amarelado; metanoto reticulado com dobras internas nos réticulos e cerdas acessórias nos esternitos abdominais.

Thrips tabaci. Comprimento do corpo 1,0 a 1,3 mm. Coloração do corpo: variável da amarelaclara, com manchas nos tergitos abdominais, ao marrom; pernas mais claras que o tórax, crescente ocelar acinzentado; asas anteriores amareladas ou levemente sombreadas, cerdas escuras (marrons ou marrom-amareladas); antena marrom, exceto segmento I, amarelo-claro, amarelado ou marrom; segmento II marrom, mais escuro que I, bases dos segmentos III e IV e segmento $\mathrm{V}$ amarelados com extremidade apical marrom. Metanoto reticulado medianamente e sem sensilo campaniforme; tergito abdominal IX sem o par de sensilos anteriores; tergito VIII com pente póstero-marginal completo e com microtríquias bem desenvolvidas; asas anteriores com 4-6 cerdas distais. 


\section{GÊNEROS DE PANCHAETOTHRIPINAE}

\section{Resumo}

Dez gêneros de Panchaetothripinae são constatados no Brasil, incluindo o registro de Oneilliella. Dentre os gêneros mais importantes economicamente, destacam-se Caliothrips, Heliothrips, Retithrips e Selenothrips. Uma chave para os gêneros é apresentada, juntamente com a caracterização morfológica dos mesmos.

\section{Summary}

Ten Panchaetothripinae genera occur in Brazil, including the record of Oneilliella. Among the most important genera are: Caliothrips, Heliothrips, Retithrips and Selenothrips. A key to the genera is presented and comments on some morphological characters are presented.

\subsection{Introdução}

Os gêneros e espécies de Panchaetothripinae foram bastante estudados do ponto de vista taxonômico (Wilson, 1975), sendo constatados 14 dos 35 gêneros dessa subfamília na região Neotropical (Nonaka \& Okajima, 1992). No Brasil, foram registrados 13 espécies em 9 gêneros (Wilson 1975, Mound \& Marullo 1996, Mound \& Monteiro 1997, Monteiro et al. 1999). Embora o gênero Oneilliella não tenha sido registrado no Brasil, uma fềmea pertencente a esse gênero, coletada no Brasil na década de 1950, encontra-se depositada na coleção do USNM. Recentemente, mais uma fêmea foi coletada na Mata de Santa Genebra, em Campinas, SP, aumentando, assim, para 10 o número de gêneros registros no País.

As caracteristícas morfológicas para reconhecimento dos gêneros de Panchaetothripinae são bem definidas. Os gêneros constatados no Brasil são: Arachisothrips, Brachyurothrips, Caliothrips, Dinurothrips, Heliothrips, Hercinothrips, Hoodothrips, Oneilliella, Retithrips e Selenothrips. Dezesseis espécies foram constatadas, a seguir 
relacionadas em ordem alfabética: Arachisothrips millsi, Brachyurothrips anomalus, Caliothrips cinctipennis, Caliothrips fasciatus, Caliothrips insularis, Caliothrips phaseoli, Dinurothrips hookeri, Dinurothrips vezenyii, Heliothrips haemorrhoidalis, Heliothrips zucchi, Hercinothrips bicinctus, Hercinothrips femoralis, Hoodothrips constrictus, Hoodothrips lineatus, Oneilliella williamsi, Retithrips syriacus e Selenothrips rubrocinctus.

\subsection{Material e Métodos}

As observações morfológicas foram realizadas nos espécimens montados em lâminas de microscopia das coleções da ESALQ/USP (Entomologia) (Apêndice 4) e do USNM, Washington, D.C. (não listadas) No caso de exemplares não montados em lâminas, inicialmente fez-se o preparo e a montagem dos espécimens, segundo o método descrito em Mound \& Ptikin, (1972) e Mound \& Manullo (1996).

A chave a separa os 10 gêneros relatados no Brasil: Arachisothrips, Brachyurothrips, Caliothrips, Dinurothrips, Heliothrips, Hercinothrips, Hoodothrips, Oneilliella, Retithrips e Selenothrips.

\subsection{Resultados e Discussão}

A subfamilia Panchaetothripinae reúne normalmente espécies com corpo de coloração escura e bastante reticulado. Muitas das espécies preferem se alimentar em tecidos foliares já desenvolvidos, ao contrário da maioria dos tripes, e apresentam movimentos bastante lentos, o que facilita acoleta e observação. Particularmente lento é Dinurothrips hookeri, que foi coletado recentemente infestando folhas de orquideas e folhas de maria-sem-vergonha. Algumas espécies passam a fase pupal no hospedeiro, como foi observado nas associações: $D$. hookeri/maria-semvergonha, $R$ syriacus/eucalipto, $S$. rubrocinctus/eucalipto e $S$. rubrocinctus/mangueira.

Várias espécies são consideradas se destacam pelos danos causados, entre elas Caliothrips phaseoli, Heliothrips hemorrhoidalis, Retithrips syriacus e Selenothrips rubrocinctus. 


\subsubsection{Chave de identificação dos gêneros de Panchaetothripinae}

1. Asas anteriores com calosidades salientes e superficie coberta com pequenos tubérculos na base, mas com microtríquias sobre a superficie na parte distal; sensilos dos segmentos antenais III e IV furcados; tarsos com 1 segmento ............................ Retithrips

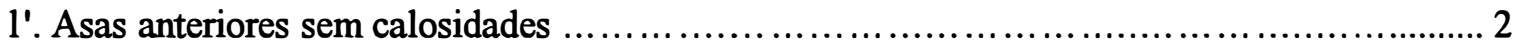

2. Sensilos dos segmentos antenais III e IV simples ................................. 3

$2^{\prime}$. Sensilos dos segmentos antenais III e IV furcados .................................... 6

3. Asas extremamente atípicas, longas, largas e bastante infladas nos $2 / 3$ basais e com reticulação poligonal conspícua; segmento antenal VIII muito longo, cerca de 3,5 vezes maior que a largura do segmento antenal II ...............................Arachisothrips

3'. Asas afiladas; segmento antenal VIII menor, cerca de 1.5 a 2.0 vezes maior que a largura do segmento antenal II ..................................................................... 4

4. Superficie do corpo quase sem reticulação, exceto parte do metanoto; asas anteriores com cerdas compridas e escuras; segmento antenal IV prolongada em um pescoço apical afilado, segmento antenal III com a parte basal longa e afilada; tergito X sem divisão longitudinal dorsalmente Brachyurothrips

4'. Superficie do corpo extremamente reticulada, incluindo a cabeça, pronoto, meso e metanoto e abdome; segmentos antenais III e IV diferentes; tergito X com divisão longitudinal completa dorsalmente 5

5. Tergito X 2.5 vezes mais longo que largo; cerdas medianas dos tergitos pequenas, distância entre suas bases maior que 5 vezes seu comprimento; retículos esculturados ao redor dos olhos compostos longos e poligonais; coloração das pernas não distintamente mais pálidas que a do corpo; asas anteriores com ápice ponteagudo e curvo em direção à cabeça; tarsos com 1 ou 2 segmentos Dinurothrips

5'. Tergito X tão longo quanto largo; cerdas medianas dos tergitos longas e bastante próximas, distância entre suas bases igual ao seu comprimento; retículos ao redor dos olhos compostos não distintos do restante da esculturação da cabeça; corpo escuro, mas pernas amareloclaras; asas anteriores com ápice arredondado; tarsos com 1 segmento Heliothrips 
6. Tergito II com numerosas microtriquias conspícuas e recurvadas ântero-lateralmente; cerdas principais das asas anteriores fusiformes e achatadas; tergito $\mathrm{X}$ assimétrico, com o lado direito mais curto que o esquerdo); cerdas medianas dos tergitos V-VII levemente curvas no ápice; tergito VIII com pente póstero-marginal completo; asas anteriores bicoloridas (3 faixas transversais claras e 2 escuras); antenas com 6 ou 8 segmentos ............ Hoodothrips

6'. Tergito II sem numerosas microtriquias conspícuas e recurvadas ântero-lateralmente; cerdas principais das asas anteriores normais; tergito $\mathrm{X}$ simétrico; antenas com 8 segmentos 7

7. Retículos da esculturação da cabeça e tórax com marcas internas; tarsos com 1 segmento; asas anteriores geralmente bicoloridas, com faixas transversais claras e escuras .. Caliothrips

7'. Retículos da cabeça e tórax sem marcas internas; tarsos com 1 ou 2 segmentos .... 8

8. Metanoto com área escura em formato de V; asas anteriores uniformemente escuras com cerdas escuras Selenothrips

8'. Metanoto sem área escura em formato de V; asas anteriores não uniformemente marromescuras 9

9. Cabeça com laterais (laterais) bastante convexas medianamente, mas constricta atrás dos olhos e na base; asas anteriores escuras, com uma pequena mancha branca próximo à base; asas anteriores com a primeira nervura com fileira de cerdas incompleta e segundo nervura, completa; tarsos com 1 segmento Oneilliela

9'. Cabeça com laterais mais ou menos paralelas; asas anteriores escuras mas com ápice pálido e com bandas ransversais claras medianas e sub-basais variavelmente desenvolvidas; asas anteriores com fileira de cerdas na primeira e segunda nervuras completa; tarsos com 2 segmentos Hercinothrips

\subsubsection{Caracterização morfológica dos gêneros de Panchaetothripinae}

Além da coloração escura do corpo, os Panchaetothripinae presentes no Brasil geralmente apresentam antena com 8 segmentos (exceto Hoodothrips brevipilus e Hoodothrips constrictus), tarsos com 1 segmento (exceto Dinurothrips vezenyii e Hercinothrips femoralis), asas afiladas e normais (exceto em Arachisothrips sp. e Retithrips sp.) e tergitos abdominais com 
linha escura (exceto Arachisothrips sp.). Os sensilos dos segmentos antenais III e IV são simples nos gêneros Arachisothrips., Brachyurothrips, Dinurothrips e Heliothrips, e furcados nos demais.

Arachisothrips. Comprimento: menor que 1,0 mm. Corpo: sem reticulação, exceto a cabeça., que é totalmente reticulada. Cabeça: palpos maxilares com 2 segmentos. Antenas: longas e bastante afiladas; 8 segmentos, segmentos III e IV com sensilos simples, segmento VIII 3,5 vezes maior que a largura do segmento II. Pronoto: cerdas pequenas. Metanoto com cerdas medianas longas. Asas anteriores bastante distintas, infladas nos $2 / 3$ basais, longas e largas, e cobertas com retículos hexagonais. Pernas: tarsos com 1 segmento e 1 garra. Abdome: com formato orbicular e esculturação fraca; tergitos sem pente póstero-marginal, $\mathrm{X}$ sem divisão longitudinal; a fêmea apresenta duas áreas glandulares grandes nos tergitos abdominais VII e VIII. A. millsi: corpo e pernas amarelo-amarronzadas; asas anteriores com mancha mais escura sub-apical; metanoto com apenas um par de cerdas (par mediano).

Brachyurothrips. Corpo: sem reticulação, exceto a parte posterior da cabeça (posterior aos olhos) e o metanoto, que é pouco reticulado. Cabeça: constricta posteriormente. Antena: 8 segmentos, III e IV com sensilos simples. Asas anteriores: marroms; cerdas longas e escuras; segunda nervura apresenta apenas uma cerda. Abdome: sem reticulação; tergitos com cerdas medianas próximas e longas; segmento IX longo. Os machos apresentam a área glandular alongada e bastante fina. As caracteristicas apresentadas pelas asas e antenas diferencia Brachyurothrips de Selenothrips, que apresenta segmentos antenais III e IV com sensilos furcados e a segunda nervura das asas anteriores com uma fileira completa.

Caliothrips. Corpo com esculturação reticulada e geralmente marcas internas dentro dos retículos. Cabeça: sem constrição posterior, laterais paralelas. Antena: 8 segmentos. Asas anteriores: geralmente apresentam faixas claras e escuras; primeira nervura de cerdas incompleta e segunda, completa; ápice afilado.

Dinurothrips. Comprimento do corpo: grande. Coloração do corpo: marrom-escura. Corpo fortemente reticulado. Cabeça: totalmente reticulada. Asas: bem desenvolvidas (macrópteros) ou não (micrópteros); cerdas pequenas. Tarsos: 1 ou 2 segmentos. Metanoto: metafurca não se estende anteriormente, em contraste com Caliothrips e Hercinothrips. Abdome: cerdas medianas 
dos tergitos pequenas e separadas; segmento $\mathrm{X}$ bastante alongado; machos apresentam área glandular em formato de "U". D. hookeri apresenta tarsos com 1 segmento e mesonoto sem divisão longitudinal mediana, enquanto que em $D$. vezenyii os tarsos apresentam 2 segmentos e mesonoto é dividido.

Heliothrips. Corpo reticulado. Cabeça reticulada, com acentuada constrição posterior e cerdas pequenas, de difícil visualização, cone bucal arredondado, palpos maxilares com 2 segmentos. Antena: segmento III e IV com sensilos simples e sem microtríquias, VIII longo e afilado. Asas anteriores: marrons e uniformes; ápice arredondado; cerdas muito pequenas, franja pósteromarginal lisa. Abdome: cerdas medianas dos tergitos próximas e medianas. $H$. haemorrhoidalis: pernas amarelas, tergitos abdominais com craspedo.

Hercinothrips. Coloração do corpo: marrom-escura. Asas anteriores: bicoloridas ou não; cerdas das nervuras longas e escuras; ápice afilado. Tarsos: dois segmentos. Cabeça: reticulada e com constrição posterior. H. femoralis: asas anteriores bicoloridas, com faixas claras e escuras, mas com ápice claro e também uma mancha sub-basal e mediana claras; ambas as nervuras com fileira completa de cerdas.

Hoodothrips. Antena: sensilos dos segmentos III e IV furcados. Abdome: tergito II com numerosas microtríquias recurvadas na parte ântero-lateral; tergito $\mathrm{X}$ com divisão longitudinal mediana incompleta. $H$. lineatus apresenta uma par de expansões em formato de acúleo nas laterais dos segmentos abdominais V, VI e VII; asas bicoloridas, com 3 faixas transversais claras e 2 escuras e cerdas das nervuras fusiformes e achatadas.

Oneilliella. Cabeça: com laterais arqueadas (mais larga após os olhos); ocelos em uma pequena elevação. Corpo (cabeça, pronoto, meso e metanotos, parte mediana dos tergitos abdominais I e II) com esculturação reticulada sem marcas internas, exceto ao longo da margem posterior da cabeça. Antena: 8 segmentos; segmentos III e IV com sensilos furcados. Asas: marromamareladas com a parte mediana e uma pequena mancha circular na base mais claras; primeira nervura de cerdas incompleta e segunda completa; ápice afilado. Tíbias com 1 segmento. Abdome: tergitos com craspedo; tergito $\mathrm{X}$ sem divisão mediana longitudinal. A única espécie do gênero foi descrita com base em uma única fêmea. 
Retithrips. Corpo pequeno e largo; antena conspícua e asas anteriores com calosidades. Segmentos antenais III e IV com sensilos furcados.

Selenothrips. Corpo de coloração escura. Corpo (cabeça, pronoto, meso e metanotos e partes medianas dos tergitos abdominas) com esculturação composta de linhas transversais formando retículos largos. Cabeça: com constrição posterior; cone bucal curto e arredondado, palpos maxilares com dois segmentos. Antena: 8 segmentos, segmentos III e IV vasiformes, com sensilos furcados e sem microtríquia. Pronoto: duas vezes mais largo que longo, com cerdas bem desenvolvidas. Asas anteriores: marrom-uniformes; ambas as nervuras com fileira completa de cerdas; franja posteriore ondulada; superficie coberta com fileiras transversais de microtríquias; ápice afilado. Pernas: tarsos com 1 segmento. Abdome: tergitos com retículos poligonais cobrindo os terços laterais; par de cerdas medianas bem desenvolvidas; microtríquias presentes póstero-medianamente nos tergitos intermediários, especialmente no VIII; tergito VIII com pente póstero-marginal completo com dentes longos; tergito $\mathrm{X}$ não dividido longitudinalmente. 
ANEXOS 


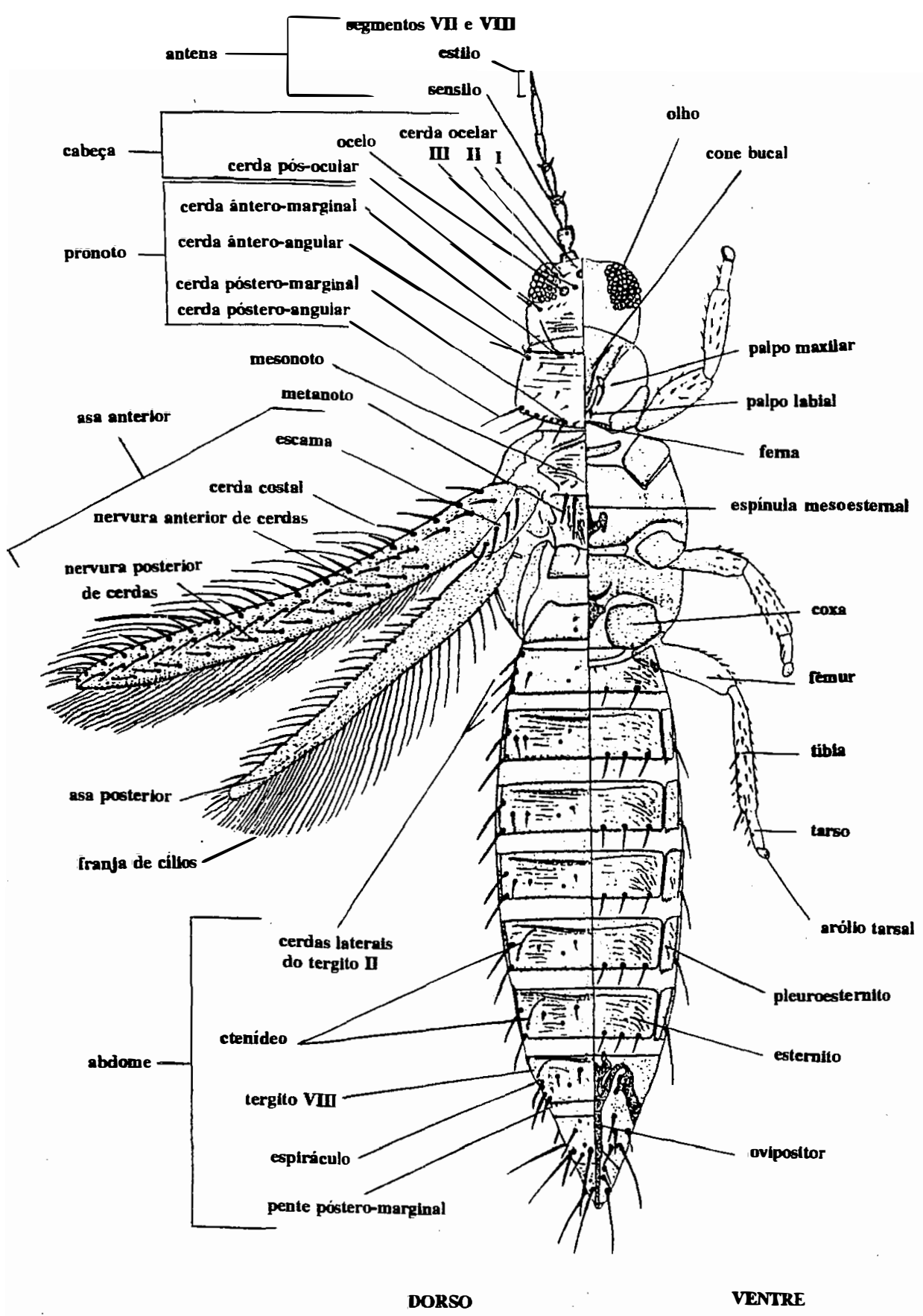

ANEXO 1. Morfologia de Thysanoptera (Terebrantia) (adaptado de Palmer et al., 1989). 


\section{ANEXO 2 \\ $\underline{\text { Terminologia morfológica }}$}

Áreas glandulares: áreas de formato, largura e comprimento variáveis, freqüentemente presentes nos esternitos abdominais dos machos.

Cerdas: processos destacáveis em forma de pêlo ou espinho, com um soquete basal.

\section{- Cerdas ocelares:}

$\mathrm{I}=$ par de cerdas anterior ao ocelo anterior.

II = par de cerdas ântero-lateral ao ocelo anterior.

III = par de cerdas entre os ocelos (dentro do triângulo ocelar) ou lateral (fora), mas caudal ao ocelo anterior $\mathrm{e}$ anterior aos ocelos posteriores.

- Cerdas pós-oculares (PO): cerdas encontradas posteriormente aos ocelos e olhos compostos, identificadas por POi, POii, POiii, POiv, POv e POvi, sendo POi o mais interno e Povi o mais externo. POiv o que se encontra normalmente desenvolvido no gênero Frankliniella.

- Cerdas principais do pronoto: o gênero Frankliniella normalmente apresenta 5 pares de cerdas desenvolvidas no pronoto, chamadas de principais:

$$
\begin{aligned}
& \text { - } \mathrm{am}=\text { cerdas ântero-marginais ( } 1 \mathrm{par}) . \\
& \text { - } \mathrm{a} \mathrm{a}=\text { cerdas ântero-angulares (lpar). } \\
& \text { - } \mathrm{pm}=\text { cerdas póstero-marginais ( } 1 \mathrm{par} \text { ). } \\
& \text { - } \mathrm{pa}=\text { cerdas póstero-angulares ( } 2 \text { pares: interno e externo). }
\end{aligned}
$$

- Cerdas discais do pronoto: incluem todas as cerdas pequenas do pronoto, com exceção daquelas na margem posterior.

\section{- Cerdas do metanoto}

- cerdas medianas: mais internas.

- cerdas laterais: mais externas.

\section{- Cerdas abdominais}

- cerdas ântero-medianas: 1-3 microcerdas na parte ântero-mediana do esternito I.

- cerdas laterais: 3 ou 4 cerdas sobre ou próximo à margem lateral do tergito II.

- cerdas $\mathrm{B}_{1}, \mathrm{~B}_{2}$ e $\mathrm{B}_{3}$ : cerdas mais desenvolvidas posteriomente nos tergitos IX eX.

- cerdas discais: par de cerdas na parte centro-mediana dos tergitos ou esternitos I-VIII. 
Craspedum: extensão membranosa da margem posterior dos tergitos e esternitos abdominais, que pode ser denteado, em forma de concha ou inteiro.

Crescente ocelar: coloração em forma crescente, que circunda cada ocelo.

Ctenídeo: fileira de microtríquias submarginais presente nos tergitos abdominais V-VIII e, algumas vezes, também no IV (vestigial).

Estrias e reticulações: marcas na cutícula; as vezes, são chamadas reticulações.

Furca: apodema localizado no meso e metatórax.

Laterais da cabeça: margem lateral da cabeça posterior aos olhos.

Microtríquia: processos da cutícula, pequenos, finos, em formato de espinho ou denteado.

Omatídeos coloridos dos olhos compostos: encontrados na parte ventral do olho, em números definidos e em um padrão distinto em cada espécie.

Pente póstero-marginal: microtríquias na margem posterior do tergito VIII, que pode ser completa ou não, com cerdas de comprimento, espessura e base variáveis.

\section{$\underline{\text { Sensilos }}$}

- Nas antenas (segmentos antenais III e IV):

- Sensilos simples: em forma de pelo

- Sensilos furcados: em forma de furca

- No metanoto: 1 par de sensilos campaniformes

- No abdome: um par medianamente nos tergitos abdominais I-VIII um ou dois pares no tergito abdominal IX um par no tergito abdominal $\mathrm{X}$

Triânqulo ocelar: triângulo formado por linhas imaginárias que unem as margens externas dos 3 ocelos. 


\section{REFERÊNCIAS BIBLIOGRÁFICAS}

Gaston, K.J. \& Mound, L.A. Taxonomy, hypothesis testing and the biodiversity crisis. Proceedings of the Royal Society of London B, v.251, p.139-142, 1933.

Heming, B.S. Order Thysanoptera. In: STEHR, F.W. Imature insects. Dubuque: Kendall/Hunt Publishing Company, 1991. v.2, cap.28, p.1-21.

Hood, J.D. J. Douglas Hood: Bibliography of scientific papers. Revista de Entomologia, vol.19, n.3, p.499-508, 1948.

International Code of Zoological Nomenclature: Code international de nomenclature zoologique. 3. Ed. London: ITZN/British Museum/UPC, 1985.

Jacot-Guillarmod, C.F. Catalogue of the Thysanoptera of the world (parts 1-4, Terebrantia; parts 5-6, Tubulifera). Annals of the Cape Provincial Museums (Natural History), vol. 7, p.11724, 1970-1979

Jacot-Guillarmod, C.F \& Brothers, D.J. Catalogue of the Thysanoptera of the world (part 7). Annals of the Cape Provincial Museums (Natural History), vol.17, n.1, p.1-94, 1986.

Lewis, T. Thrips: their biology, ecology and economic importance. London: Academic Press, 1973, 349p.

Lewis, T. An introduction to the Thysanoptera; a survey of the group. In: International Conference on Thrips, Burlington, 1989. Towards understanding Thysanoptera; 
proceeding, edited by B.L. Parker and othrs. Radnor, USDA/Forest Service/Northeastern Forest Experiment Station, p.3-22, 1991. (USDA. General Technical Report, NE-147).

Monteiro, R.C. Thrips palmi. In: E.F. Vilela, F. Cantor \& R.A Zucchi (eds.) Histórico e impacto das pragas introduzidas no Brasil, com ênfase na fruticultura (in press), 2000.

Monteiro, R.C.; Zucchi, R.A.; Mound, L.A. Record of Thrips palmi Kamy, 1925 (Thysanoptera, Thripidae) in the State of São Paulo, Brazil. Revista de Agricultura, vol. 70, n.1, p.53-55, 1995.

Monteiro, R.C.; Zucchi, R.A.; Mound, L.A. Thrips tabaci Lind.: é realmente uma praga do algodoeiro no Brasil? Anais da Sociedade Entomológica do Brasil, vol.27, n.3, p. 489494, 1998.

Monteiro, R.C.; Mound, L.A.; Zucchi, R.A. Thrips (Thysanoptera) as pests of plant production in Brazil. Revista Brasileira de Entomologia, vol.43, n.3/4 (in press), 1999.

Moritz G. Morphogenetic development of some species of the Order Thysanoptera (Insecta). Thrips Biology and Management. New York: Plenum Press Corp., Proceedings. 1995.

Moulton, D . A new species of Frankliniella from Chile. Revista Chilena de Historia Natural Pura y Aplicada, vol.30, p.9-10, 1926.

Moulton, D. Thysanoptera of South America (I). Revista de Entomologia, vol.2, n.4, p.451484, 1932.

Moulton, D. Thysanoptera of South America (II). Revista de Entomologia, vol.3, n.1, p.96$133,1933 a$.

Moulton, D. The Thysanoptera of South America (III). Revista de Entomologia, vol.3, n.2, p.277-262, 1933b.

Moulton, D. The Thysanoptera of South America (IV). Revista de Entomologia, vol.3, n.3, p.385-419, 1933 c. 
Moulton, D. The Thysanoptera of South America (Conclusion). Revista de Entomologia, vol.3, n.4, p.447-458, $1933 \mathrm{~d}$.

Moulton, D. Two new species of Thysanoptera in Argentina and records of other species. Anales de la Sociedad Cientifica Argentina, vol.12, n.6, p.254-257, 1935.

Moulton, D. Thysanoptera from Minas Geraes, Brasil. Revista de Entomologia, vol.9, n.3/4, p.374-382, 1938.

Moulton, D. Thysanoptera from Minas Geraes, Brasil (Second Paper). Revista de Entomologia, vol.12, n.1/2, p.314-322, $1941 .$.

Moulton, D. The genus Frankliniella Kamy, with keys for the determination of species (Thysanoptera). Revista de Entomologia, vol.19, n.1-2, p. 55-114, 1948.

Mound, L.A. Thrips and whitefly. In: Gibbs, A.J. (ed.). Viruses and Invertebrates. Amsterdam: North Holland Publishing Co. p.229-242, 1973.

Mound, L.A. Biological diversity. In: Lewis, T. (ed.). Thrips as crop pests. Wallingford: CAB International, 1996. cap.6, p.197-215, 1997.

Mound, L.A. \& Marullo, R. The thrips of Central and South America: an introduction (Thysanoptera: Thripidae). Memoirs on Entomology International, vol. 6, p.1-487, 1996.

Mound, L.A. \& Monteiro, R.C. A review of the genus Heliothrips (Thysanoptera; Thripidae), with a new sister-species of the greenhouse thrips from South Eastem Brazil. Journal of the New York Entomological Society, vol.105, n.3-4, p.154-160, 1997.

Mound, L.A. \& Nakahara, S. The genus Frankliniella (Thysanoptera: Thripidae): character assessment at generic and specidf levels. Journal of Pure and Applied Zoology, Zoology, vol.4, p.287-295, 1993. 
Mound, L.A. \& Ptikin, B.R. Microscopic whole mounts of thrips (Thysanoptera). Entomologist's Gazette, vol.23, n.2, p.121-125, 1972.

Mound, L.A. \& Teulon, D.A.J. Thysanoptera as phytophagous opportunists. In: Parker, B.L.; Skinner, M; Lewis, T. (eds). Thrips biology and management. New York: Plenum Publishing Corporation. p.3-20, 1995.

Mound, L.A.; Heming, B.S.; Palmer, J.M. Phylogenetic relationships between the families of recent Thysanoptera (Insecta). Zoological Journal of the Linnean Society of London, vol.69, p.111-141, 1980 .

Nakahara, S. The genus Thrips Linnaeus (Thysanoptera: Thripidae) of the new World. USDA, Technical Bulletin n. 1822, p.1-183, 1994.

Nakahara, S. Annotated list of the Frankliniella species of the World (Thysanoptera: Thripidae). Contributions on Entomology, International, vol.2, n. 4, p. 355-389, 1997.

Nonaka, T. \& Okajima, S. Two new genera and species of the subfamily Panchaetothripinae (Thysanoptera: Thripidae) from Southeast Asia. Bulletin of the Biogeographical Society of Japan, vol. 47, p.103-108, 1992.

O'Neill, K. J.Douglas Hood: bibliography os scientific papers, chiefly on thrips, and index of new names, 1948-1969. Proceedings of the Entomological Society of Washington, vol.76, n.3, p.297-309, 1974.

Palmer, J.M.; Mound, L.A.; du Heaume, G.J. Thysanoptera. Wallingford, CAB. 74p. (Cie guides to insects of importance to man, 2), 1989.

Sakimura, K. The present status of thrips-borne virus. In: Maramorosch, K. (ed.). Biological transmission of disease agents. New York: Academic Press, 1962, p.33-40.

Sakimura, K. \& K O'Neill. Frankliniella, redefinition of genus and revision of the minuta group species (Thysanoptera: Thripidae). USDA Technical Bulletin n.1572, 1979, 49p. 
Silva, A.G.A., C.R. Gonçalves, D.M. Galvão, A.J.L. Gonçalves, J. Gomes, M.N. Silva \& L. Simoni. Ordem Thysanoptera. In: Quarto catálogo de insetos que vivem nas plantas do Brasil. Rio de Janeiro, Ministério da Agricultura, t.1, p.18-33, 1968.

Takahashi, R. Metamorphosis of Thysanoptera. Zoological Magazine (Tokyo), vol.35, p.85, 1921.

Wilson, T.H. A monograph of the subfamily Panchaetothripinae (Thysanoptera: Thripidae). Memoirs of the American Entomological Institute, vol. 23: 1-354, 1975. 
APÊNDICES 


\section{APÊNDICE 1}

\section{Material examinado do gênero Frankliniella depositado \\ na coleção do Museu de Entomologia da ESALO/USP}

F. annulipes - Brasil: Piracicaba, SP, em flores rosa de Cattleya autumnalis e amarelas de Laelia glandis, 20.I.1995, L.A. Mound col. (1 fềmea).

F. brevicaulis - Brasil: Piracicaba, SP, em inflorescência de bananeira, 27.X.1993. P. De Grande col.; ?, SP, inflorescência de banana var. nanicão (danificando frutos), 26.IV.1996, Paulo col.

F. brunnea - Brasil: Paranapanema, SP, flores de crisântemo, 05.VI.1994; V. Matida col. (1 fềmea) .

F. condei - Brasil: Holambra, SP, flores de crisântemo, VI.1994, A.L. Lourenção col. (2 fềmeas); Paranapanema, SP, flores de crisântemo, 05.VI.1994, V. Matida col. (1 fềmea); Taquaritinga, SP, botão floral de "murcot", XI.1997, F.M. Isler col. (3 fềmeas).

F. distinguenda - Brasil: Holambra, SP, flores de crisântemo, VI.1994, A.L. Lourenção col. (1 fêmeas); Paranapanema, SP, flores de crisântemo, 05.VI.1994, V. Matida col. (1 fêmea).

F. fulvipes - Brasil: fumo bravo (Johan); Rio do Sul, SC, fumo, ?1997, Hokko col.; Araranguá, SC, fumo, ?1997, Hokko col.

F. gardeniae - Brasil: Mogi-Mirim, SP, flores de laranja, 15.IX.1997, O. Nakano col.; Taquaritinga, SP, botões florais de "murcot", XI.1997, F.M. Isler col. (1 fềmea).

F. hemerocallis - Brasil: Jaboticabal, SP, guizo-de-cascavel (Crotalaria incana), M. Goretii col. (1 fềmea)

F. insularis - Brasil: Campinas, SP, em flores de feijão-guandu (Cajanus cajan), VI.1993, A.L. Lourenção col.; Brasil: Piracicaba, SP, em flores e frutos de acerola, 16.III.1995, V.A. Costa col.; Piracicaba, SP, em flores vermelhas de Malvaviscus sp., 21.I.1995, L.A. Mound col. 
F. occidentalis - Brasil: Artur Nogueira, SP, folhas novas de crisântemo (mudas novas), 19.IX.1994, S.F. Bandeira col.; Atibaia, SP, flores de crisântemo, 19.V.1993, E.C. Bergmann col.; Atibaia, SP, flores de crisântemo; 03.III.1994; Atibaia, SP, flores de crisântemo, 05.VIII.1997, M. Gonçalves; Campinas, SP, flores de crisântemo, V.1992, A.L. Lourenção col.; Campinas, SP, flores de violeta africana, 17.VIII.1994, P. Caldari Jr. col.; Campinas, SP, flores de violeta africana, 17.VIII.1994, P. Caldari Jr. col.; Holambra, SP, flores de crisântemo, 1993, Agro Hokko col.; Holambra, SP, flores de crisântemo, I.1995, E. Steinberg; Holambra, SP, folhas de crisântemo, 25.VIII.1997, O. Femandes col.; Holambra, SP, flores e botões de crisântemo, IX.1997, O. Fernandes col.; Holambra, SP, folhas e flores de solidáster, I.1995, E. Steinberg col.; Holambra, SP, flores de violeta africana, V.1993, A.L. Lourenção col.; Jaguariúna, SP, folhas de crisântemo, 12.III.1994, D. Pagotto; Paranapanema, SP, 05.VII.1994, flores de crisântemo, V. Matida col.; Paranapanema, SP, frutos de pêssego, 13.X.1994, L. Wadt col.; Paranapanema, SP, folhas novas de pêssego, 13.X.1994, L. Wadt col.; Chã de Pilar, AL, crisântemo, 1998 ?.

F. peruviana - Brasil: Piracicaba, SP, folhas de gramineas, 18.I.1995, L.A. Mound \& R.C.Monteiro col. (1 fềmea).

F. rodeos - Brasil: Piracicaba, SP, inflorescências de Piper sp. (1 fềmea), 22.I.1995, L.A. Mound col.; Piraacicaba, SP, flores e folhas de abobrinha (Cucurbita pepo cv. Caserta), 12 e 14.VII.1995, R.C. Monteiro col.

F. schultzei - Brasil: Campinas, SP, folhas de algodão, 19.I.1993, R.C. Monteiro col.; Campinas, SP, folhas de soja, XI.1991, A.L. Lourenção col.; Campinas, SP, folhas de tomate (casa-devegetação), XII.1996, A.L. Lourenção; Holambra, SP, gladíolo, 31.I.1995, E. Steinberg col.; Ilha Solteira, SP, folhas novas de melancia, 14.XI.1993, C. Almeida col; Mogi-Guaçu, SP, folha de algodão, XII.1995, A.L. Lourenção; Paulínia, SP, folhas de cebola, 27.VI.1997; Piracicaba, SP, folhas de girassol, 23.VIII.1993, A.R Tavares col.; São Luís, MA, alface, 1996?, G.A. Ramos; Petrolina, PE, flores de tomate industrial, 18.VI.1996, I.B. Albert Jr. col.; Petrolina, PE, flores de melão, 19.VI.1996, I.B. Albert Jr. col.; Santa Maria da Boa Vista, PE, algodão, 11.VII.1996, I.B. Albert Jr. col.; Curaçã, BA, melancia, 10.VII.1996, I.B. Albert Jr. col.; Angélica, MS, flor de algodão, 08.III.1993, P. Riquetti col.; Dourados, MS, folhas de algodão, 26.XII.1995, Marco 
col.; Selvíria, MS, algodão, XII.1994, Papa col.; Vicentina, MS, flores de algodão, 11.XII.1993, Cido Gomes col.; Rio Verde, GO, flores de algodão; Alto Araguari, PR, folhas de algodão, 06.XII.1995, Otair col.; Assai, PE, folha de algodão, ?1995; Formosa do Oeste, PR, folhas de algodão, 11.XII.1995; Itagué, PR, folhas de algodão, 23.XI.1995, J. Rodrigues col.; Juranda, PR, folhas de algodão, 13.XII.1995; Lobato, PR, folhas de algodão, 23.XI.1995, J. Rodrigues col.; Mercedes, PR, folhas de algodão, 14.XII.1995; Nova Santa Bárbara, PR, folhas de algodão, 24.XI.1995, J. Rodrigues col.; Palotina, PR, folhas de algodão, 13.XII.1995, Otair col.; Ponta Porã, PR, folhas de algodão, 20.XII.1995, Marco col.; Santa Cecília do Pavão, PR, folhas de algodão, 24.XI.1995, J. Rodrigues col.; São José do Ivai, PR, folhas de algodão, 28.XI.1995, J. Rodrigues col.; São Sebastião da Amoreira, PR, folhas de algodão, 24.XI.1995, J. Rodrigues col.; Veru Cruz do Oeste, PR, folhas de algodão, 07.XII.1995, Otair col.; Mossoró, RN, pimentão industrial, 18.X.1997, A.V. da Silva Filho col.

F. simplex - Brasil: Piracicaba, SP, flores de orquídea, 20.I.1995, L.A. Mound e R.C. Monteiro col. (1 fềmea).

F. trinidadensis - Brasil: Piracicaba, SP, gramíneas (1 fềmea); São Pedro, SP, folhas de gramíneas, 29.I.1995, L.A. Mound col. (1 macho).

F. williamsi - Brasil: Piracicaba, SP, folhas de tomate, 09.DX.1993, T.A. Giustolin col.; Sete Lagoas, MG, em folhas de milho, VIII.1994, I. Cruz col.; Arapongas, PR, folha de milho novo, 24.DX.1996, L.F. Weber col.; Cascavel, PR, milho, 01.X.1997, M. Fogaça col.; Canoinhas, SC, bainha das folhas de milho, XI.1994, J.M. Zonta col.; Rafard, SP, pimentão, 14.VI.1991, D. Leite col.

F. zucchini - Brasil: Piracicaba, SP, folhas e flores de abobrinha (Cucurbita pepo cv. Caserta), 12 e 14.VII.1995, R.C. Monteiro col. 


\section{APÊNDICE 2}

\section{Material examinado do gênero Frankliniella depositado na colecãa do}

\section{.United States National History Museum (USNM), em Washington, D.C.}

F. annulipes - México: Brownsville, em gardênias, 22.XII.1936, Callaghan \& Pearson col. (1 fềmea) (USNM).

F. bertelsi - Brasil: Nova Teutônia, Santa Catarina, Morus nigra, 05.XI.1949, Fritz Plaumann col., Hood n 1784 (2 fêmeas) (USNM).

F. bondari - Brasil: Bahia, on Polyanthes tuberosa, Hood $\mathrm{n}^{\circ}$ 1007, Gregório Bondar col. (2 fềmeas, parátipos); EUA: Pahokee, Flórida, em tubar rose, 30.VII.1948, G.W. Dekle col. (1 fềmea); México: San Juan del Rio, bulbos de Polianthes laredo, 17.II.1945, Leary, Chapman and Walt Cary col. (1 fềmea) (USNM).

F. brevicaulis - Panamá: Juan Diaz, arbusto ao lado da estrada, 22.I.1934, James Zetek col., Hood n ${ }^{\circ} 1087$ (2 fềmeas, parátipos); Brasil: São Paulo, em frutos de banana, 12.II. 1964, J.A. Martinez col. (1 fềmea); Brasil: São Paulo, banana - danificando frutos, 15.X.1969, R.N. Williams col. (1 macho) (USNM).

F. brunnea: Panamá: Barro Colorado Island (Gatum Lake, Panama Canal), em flores de Tridax procumbens, 25.VII.1933, J.D. Hood col., Hood n ${ }^{\circ} 1012$ (2 fêmeas, parátipos da sinonímia $F$. tridacana); Guatemala: Chimaltenango, Tagetes calendula e Tithonia sp., x.1991, R.C. Lambe (1 fềmea); Venezuela: El Tocuyo, Tagetes minuta, IV.1972, R. Urtiaga (1 fềmea) (USNM).

F. condei - Brasil: Videira, Santa Catarina, flores de Prunus persica var. nuscipersica, 20.IX.1995, E.R. Hickel (2 fêmeas) (USNM).

F. desantisi - Brasil: Nova Teutônia, Santa Catarina, Myrtaceae, 10-12.X.1949, Fritz Plaumann col., Hood n 2064 (1 fềmea); Brasil: Nova Teutônia, Santa Catarina, coletando com redadas, 29.XII.1948, Hood n 2016 (1 fềmea) (USNM). 
F. distinguenda - Martinica: próximo a Fort de França, gramíneas, 14.III.1915, C.B. Williams col. (1 fêmea); Paraguai, Fiebrig col. (1 fềmea, cótipo da sinonimia F. vernoniae) (USNM).

F. fulvipennis - Brasil, Ex. coleção do Dr. Moulton (2 fềmeas, parátipos); Guatemala: interceptado em Los Angeles, Califórnia, EUA, em rosa (flor de corte), 01.III.1987, M. Richter col. (1 fềmea) (USNM).

F. fulvipes - Panamá: Chorrera, flores de Solanum subinerme, 13.VIII.1933, James Zetek col., Hood. n ${ }^{\circ} 1055$ (1 fềmea, parátipo da sinonímia F. sulfuripes); Brasil, Ex. coleção do Dr. Moulton (1 fềmea) (USNM).

F. gardeniae - Costa Rica: San Isidro, flores "Valencia", 04.XII.1995, C. Childers col. (1 macho); Costa Rica: próximo a San Carlos, V.1996, em flores de citros, C. Childers col. (1 fêmea); México: Brownsville, em gardênia, 11.III.1937, C.L. Pamell col. (1 fềmea) (USNM).

F. gemina - Uruguai: Montevidéo, em flores de Trifolium repens, 11.IV. 1945, Penacol col. (2 fềmeas); Uruguai: Montevidéo, em flores de cruciferas, 11.IV.1945, Penacol. Col. (1 fềmea); Argentina: Mendoza, "pear peach or plum", IX.1968, P. Mansur col. (1 fềmea) (USNM).

F. hemerocallis - EUA: Lake Geneva, Wisconsin, em Hemerocallis sp., 07.VII.144, E.J. Kraus col. (2 fêmeas); EUA: Glen Dale, Maryland, Hemerocallis sp., 09.IV.1969, F.F. Smith e R.E. Webb col. (1 fềmea) (USNM).

F. insularis - Brasil: Bahia, em flores de Passiflora, G. Bondar col., Hood n 758 (1 fềmea); Brasil: Bahia, em várias plantas, G. Bondar col., Hood n 1008 (1 fềmea); Brasil: 17.V.1990, V.H. Paes Bueno col. (1 macho) (USNM).

F. longipennis - Peru: Almirante, Dept. Amazonas, em flores de Fuchsia decussata, 21.XII.1936, F. Woytkowski col., Hood $\mathrm{n}^{\circ} 1171$ (2 fềmeas, parátipos da sinonímia Exophthalmothrips moultoni); Brasil: Pinhal Preto, SC, serrapilheira, XI.1957, F. Plaumann col. (1 fềmea) (USNM).

F. longispinosa - Brasil: Minas Gerais, em folhagem de trepadeira desconhecida, 06.IV.1933; Hambleton, col. (2 fêmea) (USNM). 
F. occidentalis - EUA: Spalding Co., GA, crisântemo (em casa-de-vegetação), 12.II.1998, R. Beshear col. (1 fềmea); EUA: Johnston, Iowa, Medicago sativa, 20.II.1991, J. Campbell col. (1 fêmea); EUA: Rosalia, Idaho, "barley", 26.VI.1992, N. Matteson col. (1 fêmea); EUA: Edwards Co., Texas, Hymeroxys odorata, 13.II.1991, T.0. Robins col. (1 fềmea e 1 macho); EUA: Johnston, Iowa, Glycine max, 28.II.1991. J. Campbell col. (1 fêmea); EUA: Golden Gate N.R.A., San Francisco, Califórnia, Cryophytum crystallinum, 12.VII.1992; S. Nakahara col. (1 fềmea) (USNM).

F. oxyura - Brasil: Nova Teutônia, SC, Seguieria sp., 02.II.1949, F. Plaumann col., Hood n 2033 (1 fềmea); Brasil: Nova Teutônia, SC, Sambucus niger, 04.IX.1949, F. Plaumann col., Hood ${ }^{\circ} 2055$ (USNM)

F. peruviana - Peru: Tingo Maria, inflorescências de Lonchocarpus sp., 20.IX.1944, Hambleton col. (1 fềmea) (USNM).

F. rodeos - Brasil: interceptado no aeroporto de Los Angeles, Califórnia, EUA, em flores de Chrysanthemum sp., 02.XI.1987, M. Suzuki col. (2 fềmeas); Brasil: Minas Gerais, flores de abacate, 24.IX.1932, Hambleton col. (1 fềmea); Argentina: interceptado em Brownsville, México, em alfafa (1 macho) (USNM).

F. schultzei - Brasil: Videira, SC, flores de Prunus persica var. nuscipersica, 20.IX.1995, E.R. Hickel col. (1 fềmea); Brasil: interceptado em Baltimore, Maryland, EUA, em Lactuca sativa, 01.II.1985, J.F. Cavey col. (1 macho); Brasil: Campinas, SP, em algodão, 21.IV.1936, E.J. Hambleton col., Hood n 6 (1 fềmea); Havai: Saddle Road, em Convulvulus sp. (moming glory), 29.XII.1994, S. Nakahara col. (1 fềmea) (USNM).

F. simplex - México: Pachurus?, Eupatorium sp., 14.VII.1974, Tuttle et al. col. (1 fêmea); México: interceptado em Forth Worth, Texas, EUA, em cravo, 12.V.1946, C.P. Daley col. (1 macho); México: interceptado em Forth Worth, Texas, EUA, em rosa, 23.V.1946 (1 fêmea) (USNM).

F. trinidadensis - Brasil: Nova Teutônia, SC, gramíneas mortas, VI.1957, F. Plaumann col. (1 fềmea) (USNM). 
F. varipes - Brasil: Bahia, Lantana camara, 19.VII.1972, J.A. Winder col. (1 fêmea) (USNM).

F. williamsi - Peru: Canete, em milho, 13.VII.1942, E.J. Hambleton (1 fêmea); Colômbia: Palmira, milho, 26.III.1973, O. Vargas col. (1 fêmea e lmacho); Guatemala: interceptado em Los Angeles, Califómia, EUA, haste de Asparagus officinalis, 10.XI.1985, M. Richter col. (1 fêmea) (USNM).

Frankliniella sp. nova - Brasil: Nova Teutônia, SC, 05.V.1949., Fritz Plaumann col., Hood n ${ }^{\circ}$ 2018-S (1 fềmea); Brasil: Nova Teutônia, SC, 27.V.1949, nome da planta ilegivel, Fritz Plaumann col., Hood nº 1874 (1 fềmea) (USNM).

Para a descrição da nova espécie, foram examinados os seguintes espécimens:

F. australis - Argentina: Villa Vicencio, 15.III.1921, inflorescência de T. candicans, E.E. Blanchard col. (2 fêmeas, parátipos); Argentina: Mendoza, "pear peach or plum", LX.1968, P. Mansur col. (1 fêmea); Chile: Ex. coleção do Dr. Moulton (1 fềmea, parátipo da sinonímia $F$. cestrum); Argentina: Rio Grande, Tierra del Fuego, em plantas na praia, 14.II.1951, Torres e De Santis col. (1 fêmea, sinonímia $F$. argentinae) (USNM). 


\section{APENDICE 3}

\section{Material examinado do gênero Thrips depositado}

na coleção do Museu de Entomologia, ESALQ/USP

Thrips australis - Brasil: Piracicaba, SP, folhas e flores em ramo quebrado de Eucalyptus sp. caído no chão, 28.VIII.1995, C.H.W. Flechtmann col.

Thrips palmi - Brasil: Artur Nogueira, SP, folhas novas de crisântemo (mudas novas), 28.I.1995, S.F. Bandeira; Atibaia, em tomate; Atibaia, SP, batata (folha), 08.VI.1994, H. Tsukamoto; Atibaia, SP, crisântemo; Conchal, SP, em jiló; Elias Fausto, SP, folhas e frutos novos de berinjela, 16.III.1993, O. Nakano col.; Holambra, SP, berinjela; Holambra, SP, folhas de crisântemo, 12.I.1995, Ulderico col.; Holambra, SP, folhas de pimentão, VIII.1994, A.L. Lourenção col.; Holambra, SP, flores de pimentão, VIII.1994, A.L. Lourenção col.; Itapetininga, SP, batata (folhas), R.G.A. Gurgel, 24.IX.1993; Itapira, SP, folhas e frutos de pimentão, 15.V.1994, C.E. Cavenaghi col.; Jaguariúna, SP, crisântemo; Mogi,-Guaçu, SP, folhas de crisântemo, III.1995, J. De Wit col.; Monte Mor, SP, melancia; Paranapanema, SP, berinjela, 28.VI.1994, V. Matida; Paranapanema, SP, flores de pimentão, 28.VI.1994, V. Matida col.; Paulínia, SP, berinjela; Paulínia, SP, frutos novos de pimentão, III.1994, A.L. Lourenção col.; Paulinea, SP, folhas, flores e frutos de pimentão, III.1994, A.L. Lourenção col.; Piedade, SP, berinjela (folhas e frutos), 16.III.1993, R. Agnello; Pinhalzinho, SP, batata, 01.VI.1994, L.A. Mariano; Pinhalzinho, SP, batata, 26.III.1995, E.C. Bergmann; Registro, SP, berinjela (frutos novos), XI.1992, A.L. Lourenção; Registro, SP, folhas de pepino, XI.1992, A.L. Lourenção col.; Registro, SP, folhas de vagem, XI.1992, A.L. Lourenção; São Miguel Arcanjo, SP, flores de pimentão, V.1993, O. Nakano col.; ? Marilia, SP, folhas e flores de tomate, 25.VI.1993, G. Groppo col.; Bueno Brandão, MG, batata (folha), IV.1995, A.L. Lourenção; Cachoeiras de Macacu, RJ, pimentão (estágio floração/frutificação), 21.V.1997; Vassouras, RJ, pimentão; Assai, PR, feijão-de-corda; Morretes, PR, pepino, 07.IV.1995, M.A.C. Zawadneak; Normandia, RR, melancia; Apiaú, RR, melancia; Teixeira de Freitas, BA, folhas de melancia, 05.XII.1997, R.Y. Hanyu col.; Goiânia, GO, abóbora; Goiânia, GO, berinjela; Goiânia, GO, feijão; Goiânia, GO, melancia; Goiânia, GO, pepino; Goiânia, GO, tomate; Luziânia, Go, feijão; Brasilia e arredores, DF, abóbora; Brasilia, DF, feijão. 
Thrips simplex - Brasil: Capão Bonito, SP, folhas e flores de gladíolo, A.L. Lourenção col.; Holambra, SP, flores de gladíolo, 31.I.1995, E. Steinberg col.

Thrips tabaci - Brasil: Jequié, BA, folhas de aspargo; Piracicaba, SP, folhas de cebolinha, 13.X.1993, M.T. Iamauti; Bandeirantes, PR, folhas de cebolinha, 16.X.1993, L. Mihsfeldt; Piracicaba, SP, folhas de cebola; Campinas, SP, folhas de alho, A.L. Lourenção; Campinas, SP, folhas de alho, C. Stein; Paulínia, SP, folhas de cebola, 27.VI.1997; Londrina, PR, folhas de cebola, 27.X.1993. P.M.O.J. Neves; Ituporanga, SC, folhas de cebola, 19.I.1995, P. Gonçalves. 


\begin{abstract}
APÊNDICE 4
Material examinado dos gêneros de Panchaetothripinae depositado na coleção do Museu de Entomologia da ESALQ/USP
\end{abstract}

Caliothrips phaseoli - Brasil: Bandeirantes, PR, folhas de soja, 06.XI.1993, L. Mihsfeldt; Campinas, SP, folhas de soja, XI.1991, A.L. Lourenção; Campinas, SP, folhas de soja, 19.I.1994, R.C. Monteiro col.; Ilha Solteira, SP, folhas novas de soja, 14.XI.1993, C. Almeida col.; Jaboticabal, SP, feijão, III.1992, N.M. Martinelli col.; Piracicaba, SP, folhas de girassol, 23.VIII.1993, A.R. Tavares col.; Piracicaba, SP, folhas de soja, 16.XII.1993, R.C. Monteiro col.; Piracicaba, SP, folhas de soja, 11,20 e 27.I.1994, E. Magrini col.; Santa Maria da Boa Vista, PE, algodão, 11.VII.1996, I.B. Albert Jr.

Dinurothrips hookeri - Brasil: Piracicaba, SP, folhas de orquídeas Dendrobium sp. e Phalaenopsis sp., 06.XI.1997, Carlos col.; Piracicaba, SP, maria-sem-vergonha (Impatiens balsamina, Balsaminaceae), R.C. Monteiro col.

Heliothrips haemorrhoidalis - Brasil: Campinas, SP, folhas de renda portuguesa, III.1992; A.L. Lourenção col.; Campinas, SP, folhas de samambaia-de-metro, V.1992, A.L. Lourenção col.; Campinas, SP, folhas de samambaia, XI.1993, A.L. Lourenção col.; Selvíria, MS, seringueira, 01.VI.1994, M.R. Vieira col.; Petrolina, PE, videira, 1994, F.N.P. Hagi col.

Heliothrips zucchi - Brasil: Mata de Santa Genebra, Campinas, SP, em ? Meliacea, 02.VII.1996, L.A. Mound col. É uma espécie semelhante a haemorrhoidalis, do qual diferenci-ase por apresentar os fềmures amarelos, ao invés de marrom, e segmento antenal IV menor e seu sensilo ventral mais maior. Ambas diferem de H. sylvanus por apresentarem a cabeça e o tergito $\mathrm{X}$ mais curtos, mas a cerdas medianas tergais mais longas, e em possuir cerdas na margem anterior das asas anteriores (Mound \& Monteiro, 1997).

Oneilliella sp. - Brasil: Mata de Santa Genebra, Campinas, SP, bambu, 02.VII.1996, L.A. Mound \& R.C. Monteiro col. (depositado no laboratório do Dr. Thomas, na Unicamp) 
Retithrips syriacus - Brasil: João Pinheiro, MG, em Eucalyptus sp., VII.1987, O. Peres Filho col.; Petrolina, PE, videira itália, 19.XI.1992, F.N.P. Hagi col.; Petrolina, PE, videira, 02.VIII.1993, F.N.P. Hagi col.; Petrolina, PE, videira, 03.VIII.1993, F.N.P. Hagi col.; Petrolina, PE, videira, 20.IV.1995, F.N.P. Hagi col.

Selenothrips rubrocinctus - Brasil: Campinas, SP, folhas de mangueira, X.1992, A.L. Lourenção col.; Sete Lagoas, MS, folhas de Eucalyptus urophylla, 07.IV.1994, C.F. Wilcken col.; Três Lagoas, MS, folhas de Eucalyptus urophylla, 17.VI.1994, C.F. Wilcken col.; Três Lagoas, MS, folhas de Eucalyptus urophylla, 11.VIII.1995, C.F. Wilcken col. Barro Alto, GO, seringueira, 08.VIII.1995; Inham Bupé, BA, em folhas de Eucalyptus pellita, 29.VIII.1994, C.F. Wilcken col.; Piracicaba, SP, folhas de Coleus sp., 04.IV.1991, R.C. Monteiro col; Petrolina, PE, melancia, 02.12.94, F.N.P. Hagi col.; Petrolina, PE, mangueira, 1994, F.N.P. Hagi col.; Manaus, AM, folhas de mangostão (Garcinia mangostana), 15.III.1995, M.V.B. Garcia col. 